\title{
CFD Investigation of the Atmospheric Boundary Layer under Different Thermal Stability Conditions
}

\author{
Jacobus Erasmus Pieterse
}

Thesis presented in partial fulfilment of the requirements for the degree of Master of Science in Engineering (Mechanical) in the Faculty of Engineering at Stellenbosch University

Supervisor: Prof T.M. Harms

March 2013 


\section{DECLARATION}

By submitting this thesis electronically, I declare that the entirety of the work contained therein is my own, original work, that I am the sole author thereof (save to the extent explicitly otherwise stated), that reproduction and publication thereof by Stellenbosch University will not infringe any third party rights and that I have not previously in its entirety or in part submitted it for obtaining any qualification. 


\begin{abstract}
An accurate description of the atmospheric boundary layer (ABL) is a prerequisite for computational fluid dynamic (CFD) wind studies. This includes taking into account the thermal stability of the atmosphere, which can be stable, neutral or unstable, depending on the nature of the surface fluxes of momentum and heat. The diurnal variation between stable and unstable conditions in the Namib Desert interdune was measured and quantified using the wind velocity and temperature profiles that describe the thermally stratified atmosphere, as derived by MoninObukhov similarity theory. The implementation of this thermally stratified atmosphere into CFD has been examined in this study by using Reynoldsaveraged Navier-Stokes (RANS) turbulence models. The maintenance of the temperature, velocity and turbulence profiles along an extensive computational domain length was required, while simultaneously allowing for full variation in pressure and density through the ideal gas law. This included the implementation of zero heat transfer from the surface, through the boundary layer, under neutral conditions so that the adiabatic lapse rate could be sustained. Buoyancy effects were included by adding weight to the fluid, leading to the emergence of the hydrostatic pressure field and the resultant density changes expected in the real atmosphere. The CFD model was validated against measured data, from literature, for the flow over a cosine hill in a wind tunnel. The standard $k-\varepsilon$ and SST $k-\omega$ turbulence models, modified for gravity effects, represented the data most accurately. The flow over an idealised transverse dune immersed in the thermally stratified $\mathrm{ABL}$ was also investigated. It was found that the flow recovery was enhanced and re-attachment occurred earlier in unstable conditions, while flow recovery and re-attachment took longer in stable conditions. It was also found that flow acceleration over the crest of the dune was greater under unstable conditions. The effect of the dune on the flow higher up in the atmosphere was also felt at much higher distances for unstable conditions, through enhanced vertical velocities. Under stable conditions, vertical velocities were reduced, and the influence on the flow higher up in the atmosphere was much less than for unstable or neutral conditions. This showed that the assumption of neutral conditions could lead to an incomplete picture of the flow conditions that influence any particular case of interest.
\end{abstract}




\section{OPSOMMING}

'n Akkurate beskrywing van die atmosferiese grenslaag (ABL) is 'n voorvereiste vir wind studies met berekenings-vloeimeganika (CFD). Dit sluit in die inagneming van die termiese stabiliteit van die atmosfeer, wat stabiel, neutraal of onstabiel kan wees, afhangende van die aard van die oppervlak vloed van momentum en warmte. Die daaglikse variasie tussen stabiele en onstabiele toestande in die Namib Woestyn interduin is gemeet en gekwantifiseer deur gebruik te maak van die wind snelheid en temperatuur profiele wat die termies gestratifiseerde atmosfeer, soos afgelei deur Monin-Obukhov teorie, beskryf. Die implementering van hierdie termies gestratifiseerde atmosfeer in CFD is in hierdie studie aangespreek deur gebruik te maak van RANS turbulensie modelle. Die handhawing van die temperatuur, snelheid en turbulensie profiele in die lengte van 'n uitgebreide berekenings domein is nodig, en terselfdertyd moet toegelaat word vir volledige variasie in die druk en digtheid, deur die ideale gaswet. Dit sluit in die implementering van zero hitte-oordrag vanaf die grond onder neutrale toestande sodat die adiabatiese vervaltempo volgehou kan word. Drykrag effekte is ingesluit deur die toevoeging van gewig na die vloeistof, wat lei tot die ontwikkeling van die hidrostatiese druk veld, en die gevolglike digtheid veranderinge, wat in die werklike atmosfeer verwag word. Die CFD-model is gevalideer teen gemete data, vanaf die literatuur, vir die vloei oor 'n kosinus heuwel in 'n windtonnel. Die standaard $k-\varepsilon$ en SST $k-\omega$ turbulensie modelle, met veranderinge vir swaartekrag effekte, het die data mees akkuraat voorgestel. Die vloei oor 'n geïdealiseerde transversale duin gedompel in die termies gestratifiseerde ABL is ook ondersoek. Daar is bevind dat die vloei herstel is versterk en terug-aanhegging het vroeër plaasgevind in onstabiele toestande, terwyl vloei herstel en terug-aanhegging langer gevat het in stabiele toestande. Daar is ook bevind dat vloei versnelling oor die kruin van die duin groter was onder onstabiele toestande. Die effek van die duin op die vloei hoër op in die atmosfeer is ook op hoër afstande onder onstabiele toestande gevoel, deur middel van verhoogte vertikale snelhede. Onder stabiele toestande, is vertikale snelhede verminder, en die invloed op die vloei hoër op in die atmosfeer was veel minder as vir onstabiel of neutrale toestande. Dit het getoon dat die aanname van neutrale toestande kan lei tot 'n onvolledige beeld van die vloei toestande wat 'n invloed op 'n bepaalde geval kan hê. 


\section{ACKNOWLEDGEMENTS}

The author would like to thank Professor T.M. Harms for his supervision and guidance as well as the staff and students at Gobabeb Research and Training Centre, Namibia for facilitating the study. Namibia is a beautiful place and it was a privilege to be able to work there.

Further thanks go to the Stellenbosch University Hope Project for providing funding for this work. Thanks also to E.C.J Joubert for assisting in the collection of the wind mast data for the interdune region, as well as the site photographs.

Finally, thank you to my friends and family for their constant support. 


\section{TABLE OF CONTENTS}

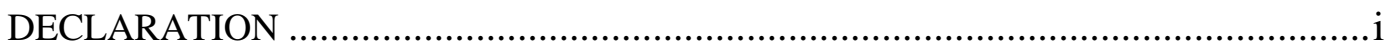

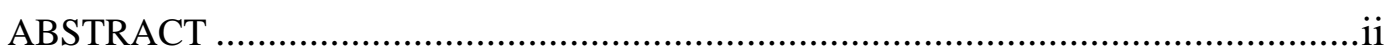

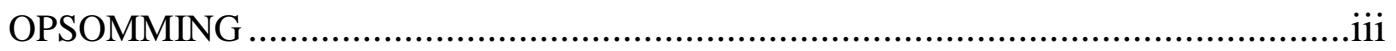

ACKNOWLEDGEMENTS ….........................................................................

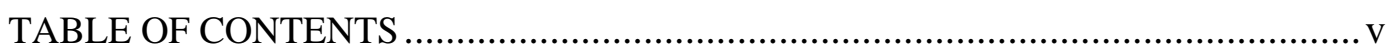

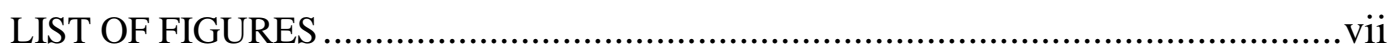

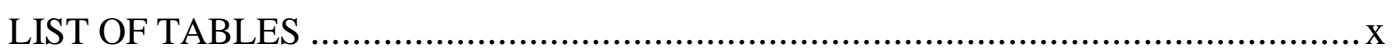

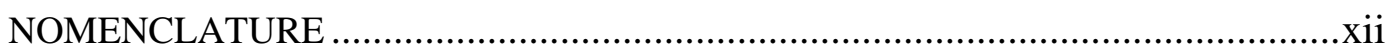

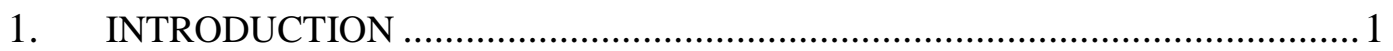

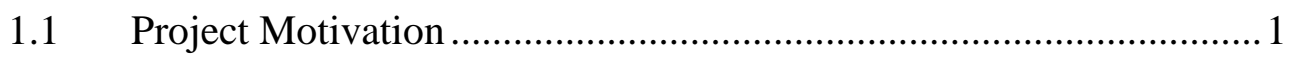

$1.2 \quad$ Project Objectives and Outline ...................................................... 3

2. A REVIEW OF ABL THEORY IN LITERATURE ...................................5

3. A REVIEW OF THE CFD MODELLING OF THE TURBULENT ABL .14

4. WIND DATA ACQUISITION AND ANALYSIS …...............................22

4.1 Wind Measurement....................................................................22

4.2 Determination of Velocity and Temperature Profiles from Data .....24

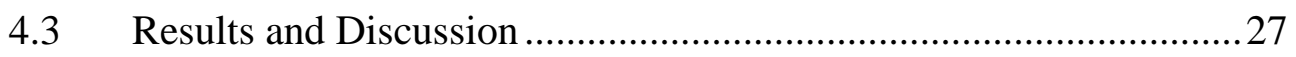

5. CFD SIMULATION OF THE ABL UNDER DIFFERENT THERMAL

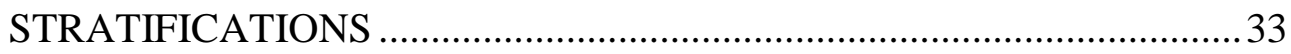

5.1 Computational Domain and Grid Generation...................................33

5.2 Flow Physics Solution Specification ...............................................36

5.2.1 Physics models and solver .....................................................

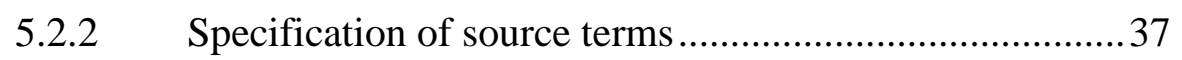

5.2.3 Boundary conditions ..................................................4 41

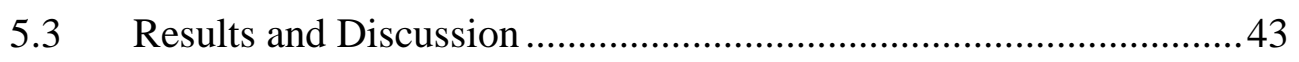

6. CFD SIMULATION OF FLOW OVER A COSINE HILL.........................58

6.1 Computational Domain and Grid Generation...................................59

6.2 Flow Physics Solution Specification ............................................61

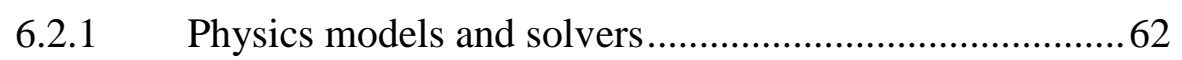

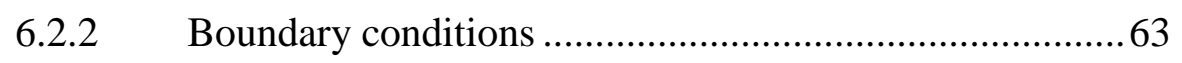

6.3 Comparison of Measurements with CFD Results ...........................64 
6.4 Results and Discussion 65

7. CFD SIMULATION OF STRATIFIED ABL FLOW OVER AN IDEALISED TRANSVERSE DUNE

7.1 Computational Domain and Grid Generation.................................69

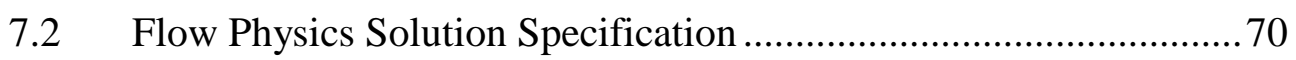

7.2.1 Physics models and solvers ............................................... 70

7.2.2 Boundary conditions .................................................... 72

7.3 Comparison of Measurements with CFD Results ...........................73

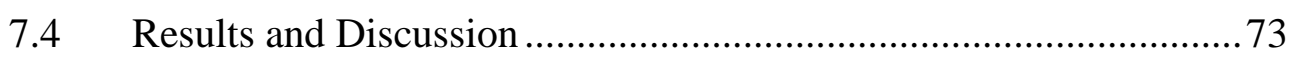

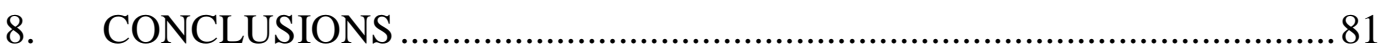

APPENDIX A: MATLAB CODE FOR DETERMINATION OF SURFACE

FLUXES FROM WIND MAST DATA …………..................... 83

APPENDIX B: WIND PROFILE DATA SAMPLE CALCULATION ...............87

APPENDIX C: NATURAL CONVECTION BUOYANCY DRIVEN FLOW ....99

APPENDIX D: GRID SENSITIVITY ANALYSIS FOR COSINE HILL

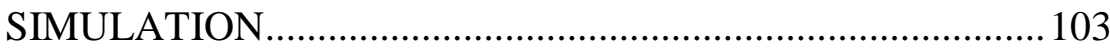

APPENDIX E: GRID SENSITIVITY ANALYSIS FOR TRANSVERSE DUNE

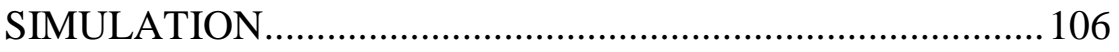

APPENDIX F: FURTHER SST K- $\Omega$ RESULTS FOR CFD SIMULATION OF THE ABL UNDER DIFFERENT THERMAL STRATIFICATIONS

APPENDIX G: RESULTS OF TURBULENT VISCOSITY FOR FLOW OVER

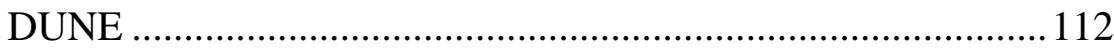

APPENDIX H: WIND SENSOR SPECIFICATIONS .................................... 114

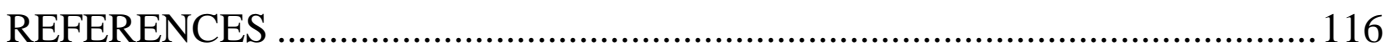




\section{LIST OF FIGURES}

Figure 2-1: Schematic of stability categories on the basis of temperature

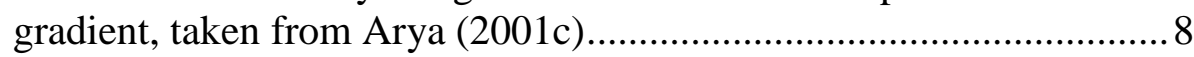

Figure 4-1: Interdune site with wind mast ................................................... 23

Figure 4-2: Wind speed and temperature sensor pair on wind mast....................2 23

Figure 4-3: Wind mast positioned on the interdune …....................................... 24

Figure 4-4: Measured data and predicted profiles for velocity ............................. 30

Figure 4-5: Measured data and predicted profiles for temperature …...................31

Figure 5-1: Schematic of computational domain................................................ 34

Figure 5-2: Section of computational mesh used in ABL simulation.....................36

Figure 5-3: Scaled profiles for flow properties at the domain inlet: (a) velocity; (b) turbulent kinetic energy; (c) turbulent kinetic energy dissipation rate; (d) density; (e) temperature and; (f) pressure. ............................ 44

Figure 5-4: Neutral CFD $k$ - $\varepsilon$ model results illustrating streamwise gradients for (a) wind speed $u$; (b) turbulent kinetic energy $k$; and (c) turbulent dissipation rate $\varepsilon$

Figure 5-5: Neutral CFD $k-\varepsilon$ model results illustrating streamwise gradients for (a) temperature $T$; (b) density $\rho$; and (c) pressure $P$. Theoretical values for the neutral atmosphere also shown.

Figure 5-6: Neutral CFD $k-\omega$ model results illustrating streamwise gradients for (a) wind speed $u$; (b) turbulent kinetic energy $k$; and (c) specific dissipation rate $\omega$.

Figure 5-7: Neutral CFD $k-\omega$ model results illustrating streamwise gradients for (a) temperature $T$; (b) density $\rho$; and (c) pressure $P$. Theoretical values for the neutral atmosphere also shown.

Figure 5-8: Relative change of the flow parameters for the $k$ - $\varepsilon$ and $k-\omega$ turbulence models relative to the inlet for neutral conditions.

Figure 5-9: Stable CFD $k-\varepsilon$ model results illustrating streamwise gradients for (a) wind speed $u$; (b) turbulent kinetic energy $k$; and (c) turbulent dissipation rate $\varepsilon$ 
Figure 5-10: Stable CFD $k$ - $\varepsilon$ model results illustrating streamwise gradients for (a) temperature $T$; (b) density $\rho$; and (c) pressure $P$.

Figure 5-11: Relative change of the flow parameters for the $k$ - $\varepsilon$ and $k-\omega$ turbulence models relative to the inlet for stable conditions.

Figure 5-12: Unstable CFD $k-\varepsilon$ model results illustrating streamwise gradients for (a) wind speed $u$; (b) turbulent kinetic energy $k$; and (c) turbulent dissipation rate $\varepsilon$.

Figure 5-13: Unstable CFD $k-\varepsilon$ model results illustrating streamwise gradients for (a) temperature $T$; (b) density $\rho$; and (c) pressure $P$.

Figure 5-14: Relative change of the flow parameters for the $k-\varepsilon$ and $k-\omega$ turbulence models relative to the inlet for unstable conditions.

Figure 6-1: Computational grid: (a) section through centre of hill looking down $x$-direction in $z-y$ plane; (b) looking at symmetry plane down $y$ direction in $x-z$ plane.

Figure 6-2: Comparison of the horizontal velocity results against measurement.. 66

Figure 6-3: Comparison of the turbulent kinetic energy results against measurement

Figure 7-1: Computational mesh for dune simulations .71

Figure 7-2: CFD horizontal velocity results for the two turbulence models under neutral conditions versus measurement at six locations from $-8 h$ to $10 h$ ((a)-(f)) respectively.

Figure 7-3: Horizontal velocity distribution for the two turbulence models for stable and unstable conditions at different locations from $-8 h$ to $10 h$ ((a)-(f)) respectively.

Figure 7-4: Contour plots of horizontal (streamwise) velocity for (a) neutral conditions; (b) stable conditions and; (c) unstable conditions.

Figure 7-5: Contour plots of vertical velocity for (a) neutral conditions; (b) stable conditions and; (c) unstable conditions

Figure B-1: Least square fitting of unstable Richardson number function versus geometric mean height

Figure B-2: Least square fitting of stable Richardson number function versus geometric mean height. 
Figure B-3: Least square fitting of flux profile relation (modified log law) to observed unstable mean potential temperature profile

Figure B-4: Least square fitting of flux profile relation (modified log law) to observed stable mean potential temperature profile

Figure B-5: Least square fitting of flux profile relation (modified log law) to observed unstable mean velocity profile.

Figure B-6: Least square fitting of flux profile relation (modified log law) to observed stable mean velocity profile.

Figure C-1: Geometry of computational domain (Scale 1:10) .95

Figure C-2: Computational grid. 96

Figure C-3: Steady state wall shear stress on vertical plate. 100

Figure C-4: Steady state wall heat flux on vertical plate 100

Figure C-5: Development of steady state vertical velocity boundary layer 101

Figure C-6: Development of steady state temperature boundary layer

Figure F-1: Stable CFD $k$ - $\omega$ model results illustrating streamwise gradients for

(a) temperature $T$; (b) density $\rho$; and (c) pressure $P$. 108

Figure F-2: Stable CFD $k$ - $\omega$ model results illustrating streamwise gradients for (a) wind speed $u$; (b) turbulent kinetic energy $k$; and (c) specific dissipation rate $\omega$..

Figure F-3: Unstable CFD $k$ - $\omega$ model results illustrating streamwise gradients for (a) wind speed $u$; (b) turbulent kinetic energy $k$; and (c) specific dissipation rate $\omega$..

Figure F-4: Unstable CFD $k$ - $\omega$ model results illustrating streamwise gradients for (a) temperature $T$; (b) density $\rho$; and (c) pressure $P$.

Figure G-1:Turbulent viscosity for the two turbulence models under neutral conditions at the measurement locations from $-8 h$ to $10 h$ ((a)-(f)) respectively.

Figure G-2: Turbulent viscosity distribution for the two turbulence models for stable and unstable conditions at different locations from $-8 h$ to $10 h$ ((a)-(f)) respectively. 


\section{LIST OF TABLES}

Table 3-1: Original $k$ - $\varepsilon$ model constants

Table 4-1: Processed wind mast data for stable atmospheric wind blowing from Southerly direction

Table 4-2: Processed wind mast data for unstable atmospheric wind blowing from Southerly and Northerly directions .......................................... 29

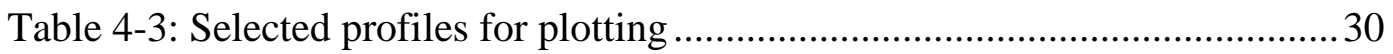

Table 5-1: Parameters describing the different stratified atmospheres.................. 33

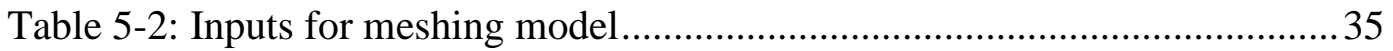

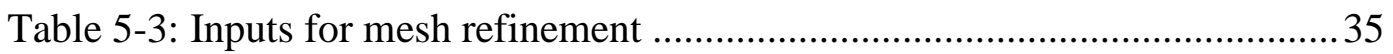

Table 5-4:Under-relaxation factors for flow simulations ................................... 37

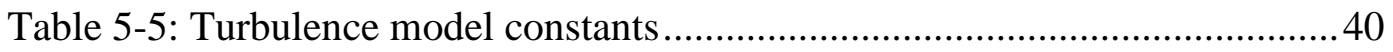

Table 5-6: Coefficients for $C \varepsilon 3$ (Alinot and Masson 2002) .................................4 41

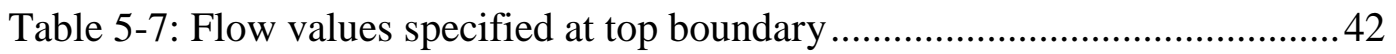

Table 6-1: Parameters derived from neutral wind tunnel incident flow ................59

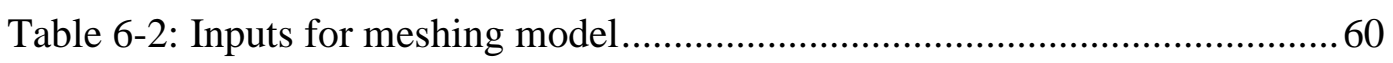

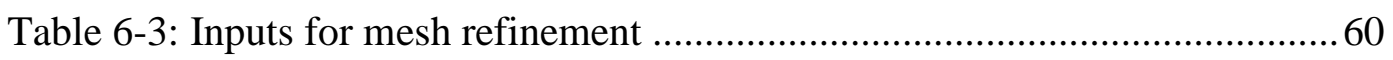

Table 6-4:Under-relaxation factors for flow simulations ....................................62

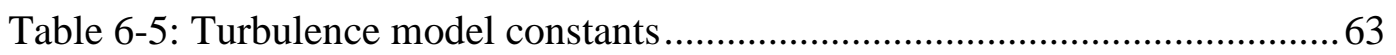

Table 6-6: Hit rate values for simulations over cosine hill ................................67

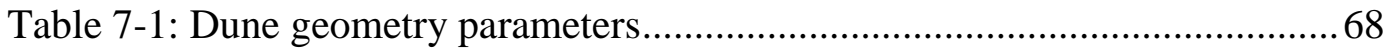

Table 7-2: Parameters describing the different stratified atmospheres.................68

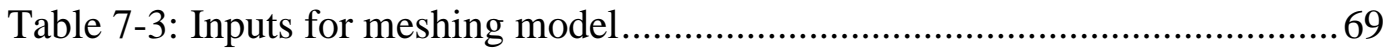

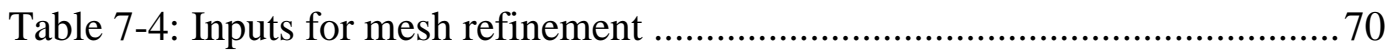

Table 7-5:Under-relaxation factors for flow simulations .................................. 71

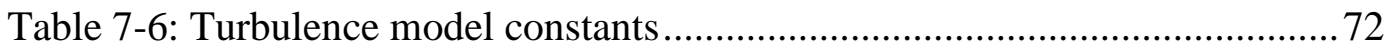


Table 7-7: Flow values specified at top boundary

Table 7-8: Results of hit rate analysis with wind tunnel data for neutral atmosphere..... 76

Table 7-9: Flow re-attachment lengths for different stability conditions 77

Table B-1: Mean temperature and velocity data at different heights. 87

Table B-2: Potential temperatures at each measurement height. .87

Table B-3: Richardson numbers for the two profiles 88

Table B-4: Results of analysis 94

Table C-1: Properties used in calculation 97

Table C-2: Under-relaxation factors for flow simulation 98

Table C-3: Calculated Prandtl and Grashof numbers 99

Table C-4: Mean steady state shear stress and heat flux 99

Table D-1: Results of discretisation error calculation for cosine hill simulation 105

Table E-1: Results of discretisation error calculation for dune simulation 107

Table F-1: Sensor calibration coefficients for logger, station 1 114

Table F-2: Sensor calibration coefficients for logger, station 2 114

Table F-3: Data-logger specifications. 114

Table F-4: Temperature sensor specifications 114

Table F-5: Wind vane specifications .115

Table F-6: Wind speed sensor specifications 115 


\section{NOMENCLATURE}

\begin{tabular}{|c|c|}
\hline$A$ & Area \\
\hline$a_{n}$ & Coefficient for $C_{\varepsilon 3}$ \\
\hline$C_{b 1}$ & Spalart-Allmaras turbulence model constant \\
\hline$C_{b 2}$ & Spalart-Allmaras turbulence model constant \\
\hline$C_{D E S k \omega}$ & DES turbulence model constant \\
\hline$C_{D E S k \varepsilon}$ & DES turbulence model constant \\
\hline$C_{D E S t}$ & DES turbulence model constant \\
\hline$C_{\text {prod }}$ & Spalart-Allmaras turbulence model constant \\
\hline$C_{v 1}$ & Spalart-Allmaras turbulence model constant \\
\hline$C_{w 2}$ & Spalart-Allmaras turbulence model constant \\
\hline$C_{w 3}$ & Spalart-Allmaras turbulence model constant \\
\hline$C_{\mu}$ & $\mathrm{k}-\varepsilon$ turbulence model constant \\
\hline$C_{\varepsilon 1}$ & $\mathrm{k}-\varepsilon$ turbulence model constant \\
\hline$C_{\varepsilon 2}$ & $\mathrm{k}-\varepsilon$ turbulence model constant \\
\hline$C_{\varepsilon 3}$ & $\mathrm{k}-\varepsilon$ turbulence model constant \\
\hline$c_{p}$ & Specific heat at constant pressure \\
\hline$c_{v}$ & Specific heat at constant volume \\
\hline$e$ & Error \\
\hline$f$ & Grid refinement factor \\
\hline$G_{b}$ & Buoyant production of turbulence \\
\hline$G_{k}$ & Shear production of turbulence \\
\hline$G r$ & Grashof number \\
\hline$g$ & Gravitational acceleration \\
\hline$H$ & Height of hill \\
\hline$h$ & Height of dune \\
\hline$h_{i}$ & Representative grid size \\
\hline$h_{0}$ & Obstruction height \\
\hline$I$ & Turbulence intensity \\
\hline$j$ & Representative grid size \\
\hline$K_{m}$ & Kinematic turbulent viscosity \\
\hline
\end{tabular}




\begin{tabular}{|c|c|}
\hline$k$ & Turbulent kinetic energy \\
\hline$k_{s}$ & Equivalent sand-grain roughness height \\
\hline$L$ & Monin-Obukhov length \\
\hline$L_{w}$ & Wall length \\
\hline$l$ & Turbulent length scale \\
\hline$l_{\infty}$ & Asymptotic length \\
\hline$M$ & Molecular weight \\
\hline$m$ & Power law exponent \\
\hline$N_{i}$ & Number of cells in grid \\
\hline$n$ & Cosine director (unit vector) \\
\hline$P$ & Pressure \\
\hline$P_{0}$ & Surface or reference pressure \\
\hline $\operatorname{Pr}$ & Prandtl number \\
\hline$p$ & Apparent order in grid convergence \\
\hline$q$ & Term in Richardson extrapolation \\
\hline$\dot{q}$ & Heat flux \\
\hline$\dot{q_{0}}$ & Surface heat flux \\
\hline$\dot{q}$ & Wall heat flux \\
\hline$R$ & Gas constant for air \\
\hline$R e$ & Reynolds number \\
\hline$R i$ & Richardson number \\
\hline$R_{b}$ & Radius of cosine hill at base \\
\hline$r$ & Radius \\
\hline$r_{i}$ & Refinement factor \\
\hline$S_{i}$ & Hypothetical vector \\
\hline$S_{m}$ & Momentum source \\
\hline$S_{k}$ & Turbulent kinetic energy source \\
\hline$S_{u}$ & Energy source \\
\hline$S_{\varepsilon}$ & Turbulent kinetic energy dissipation rate source \\
\hline$T$ & Temperature \\
\hline$T_{i}$ & Temperature at measurement height \\
\hline$T_{0}$ & Surface temperature \\
\hline
\end{tabular}




\begin{tabular}{|c|c|}
\hline$T_{w}$ & Wall temperature \\
\hline$T_{\infty}$ & Free stream temperature \\
\hline$u$ & Horizontal wind velocity \\
\hline$u_{i}$ & Velocity at measurement height \\
\hline$u_{H}$ & Velocity at height of hill \\
\hline$u_{h}$ & Velocity at height of dune \\
\hline$u_{m}$ & Mean velocity \\
\hline$u_{r}$ & Reference horizontal wind velocity \\
\hline$u_{\delta}$ & Velocity at boundary layer height \\
\hline$u_{*}$ & Friction velocity \\
\hline$u^{\prime}$ & Horizontal velocity fluctuation \\
\hline$V$ & Volume \\
\hline$v^{\prime}$ & Lateral velocity fluctuation \\
\hline$w^{\prime}$ & Vertical velocity fluctuation \\
\hline$x$ & Horizontal coordinate \\
\hline$Y_{m}$ & Turbulence production due to compressibility \\
\hline$y$ & Lateral coordinate \\
\hline$z$ & Vertical coordinate \\
\hline$z_{i}$ & Measurement height \\
\hline$z^{+}$ & Dimensionless wall distance $\left(\rho u_{*} z / \mu\right)$ \\
\hline$z_{m}$ & Geometric mean height \\
\hline$z_{r}$ & Reference height \\
\hline$z_{0}$ & Roughness length \\
\hline$z_{p}$ & Height of first cell centroid above wall \\
\hline$\alpha$ & Flow variable \\
\hline$\alpha_{1}$ & Constant if SST $k-\omega$ turbulence model \\
\hline$\beta$ & Volumetric expansion coefficient \\
\hline$\beta_{1}$ & Constant in SST $k-\omega$ turbulence model \\
\hline$\beta_{*}$ & Constant in SST $k-\omega$ turbulence model \\
\hline$\beta_{2}$ & Constant in SST $k-\omega$ turbulence model \\
\hline$\Gamma$ & Adiabatic lapse rate \\
\hline
\end{tabular}




\begin{tabular}{|c|c|}
\hline$\gamma$ & Heat capacity ratio \\
\hline$\delta$ & Boundary Layer height \\
\hline$\delta_{i j}$ & Kronecker delta \\
\hline$\varepsilon$ & Turbulent kinetic energy dissipation rate \\
\hline$\zeta$ & Stability parameter \\
\hline$\zeta_{r}$ & Stability parameter at reference height \\
\hline$\theta$ & Potential temperature \\
\hline$\theta_{0}$ & Surface potential temperature \\
\hline$\kappa$ & von Karman constant \\
\hline$\lambda$ & Thermal conductivity \\
\hline$\mu$ & Molecular dynamic viscosity \\
\hline$\mu_{t}$ & Turbulent dynamic viscosity \\
\hline$\rho$ & Density \\
\hline$\sigma_{T}$ & Turbulent Prandtl number \\
\hline$\sigma_{i j}$ & Difference in flow variable $(\alpha)$ between coarser and finer grid \\
\hline$\sigma_{k}$ & $\mathrm{k}-\varepsilon$ turbulence model constant \\
\hline$\sigma_{k 1}$ & Constant in SST $k-\omega$ turbulence model \\
\hline$\sigma_{k 2}$ & Constant in SST $k$ - $\omega$ turbulence model \\
\hline$\sigma_{v}$ & Spalart-Allmaras turbulence model constant \\
\hline$\sigma_{\omega 1}$ & Constant in SST $k$ - $\omega$ turbulence model \\
\hline$\sigma_{\omega 2}$ & Constant in SST $k-\omega$ turbulence model \\
\hline$\sigma_{\varepsilon}$ & $\mathrm{k}-\varepsilon$ turbulence model constant \\
\hline$\tau$ & Shear stress \\
\hline$\tau_{0}$ & Surface shear stress \\
\hline$\tau_{w}$ & Wall shear stress \\
\hline$\varphi_{\varepsilon}$ & Scaling parameter for turbulent dissipation rate \\
\hline$\varphi_{h}$ & Similarity function for temperature \\
\hline$\varphi_{m}$ & Similarity function for momentum \\
\hline$\psi_{h}$ & Integrated similarity function for temperature \\
\hline$\psi_{m}$ & Integrated similarity function for momentum \\
\hline$\omega$ & Specific dissipation rate \\
\hline
\end{tabular}




$\begin{array}{cl}\text { ABL } & \text { Atmospheric boundary layer } \\ \text { CAD } & \text { Computer aided design } \\ \text { CFD } & \text { Computational fluid dynamics } \\ \text { CFL } & \text { Courant-Friedrichs-Lewy } \\ \text { CSV } & \text { Coma separated values } \\ \text { DES } & \text { Detached eddy simulation } \\ \text { GCI } & \text { Grid convergence index } \\ \text { LES } & \text { Large eddy simulation } \\ \text { LR } & \text { Lapse rate } \\ \text { PBL } & \text { Planetary boundary layer } \\ \text { PIV } & \text { Particle image velocimetry } \\ \text { RANS } & \text { Reynolds averaged Navier-Stokes } \\ \text { RNG } & \text { Renormalisation group } \\ \text { SIMPLE } & \text { Semi-implicit method for pressure linked equations } \\ \text { SST } & \text { Shear stress transport } \\ \text { STL } & \text { Stereolithography }\end{array}$




\section{INTRODUCTION}

\subsection{Project Motivation}

The greater part of human endeavour occurs on the surface of our planet. It is characterised by the interaction between the Earth's rocky crust on the continents, and the water of the oceans, with the lower atmosphere. A thorough understanding of the processes taking place in this interface layer is of utmost concern to humans, from weather and climate prediction, to pollution studies, and the interactions of the wind on our structures and power stations. The lower 1-2 km of the troposphere is characterised by an exchange of momentum, heat and matter with the surface and the generation of atmospheric turbulence. This layer is known as the atmospheric boundary layer (ABL), or just as frequently, the planetary boundary layer (PBL) (Azad 1993, Blackadar 1997, Kröger 2004).

Large vertical gradients in the wind velocity, air temperature and humidity occur in the ABL, with the vertical exchanges of properties occurring mainly through turbulent motions (Arya 2001c). This turbulence is generated by mechanical means through surface friction and wind shear, and convectively due to surface heating and buoyancy. The action of turbulence results in nearly uniform distribution of particulates throughout this layer. For this reason it is commonly called the mixed layer and sometimes the friction layer (Blackadar 1997, Kröger 2004).

The specific structure of ABL turbulence is strongly influenced by the daily cycle of surface heating and cooling, the horizontal variability in surface properties and the presence of clouds (Garratt 1994). Strong radiative heating of the ground by the sun results in heat transfer to the air and the formation of the convective or unstable ABL, with buoyancy forces that tend to destabilise displaced air parcels. The unstable ABL is characterised by a near-surface superadiabatic layer (Garratt 1994). A stable ABL results when the ground cools, and heat is transferred from the air to the ground (usually under nocturnal conditions), with the formation of temperature inversions close to the surface (Garratt 1994). Neutral conditions only result when there is no heat transfer between the air and the ground, and buoyancy effects are absent.

The height of the ABL also varies in response to these diurnal cycles, with its lowest typical value of the order of $100 \mathrm{~m}$ (range of $20 \mathrm{~m}$ to $500 \mathrm{~m}$ ) in the morning to its highest value of $1 \mathrm{~km}$ (range of $0.2 \mathrm{~km}$ to $5 \mathrm{~km}$ ) during the late afternoon (Arya 2005). The velocity and temperature profiles that characterise the atmosphere under these different stability conditions are best described by MoninObukhov similarity theory (Arya 2001c), which also describes the fluxes of heat and momentum at the surface.

The accurate computational fluid dynamic (CFD) simulation of the ABL is becoming increasingly important. CFD is a tool which is increasingly being used 
to study a wide variety of processes in the ABL, where its accurate modelling is an imperative precondition in computational wind engineering (Kim and Boysan 1999, Blocken et al. 2007b, Hargreaves and Wright 2007, Yang et al. 2008). These numerical simulations can be performed by using either the Reynoldsaveraged Navier-Stokes (RANS) equations or by conducting large-eddy simulations (LES). LES generally provides more accurate solutions for the flow field but are at least one order of magnitude more computationally expensive than RANS (Rodi 1997). For this reason practical simulation of ABL flows often employ RANS in combination with two-equation turbulence models, the standard $k$ - $\varepsilon$ turbulence model being one of the most popular (Parente et al. 2011a).

As a consequence it is worthwhile investigating the simulation of the ABL under the influence of surface heat flux using these models. Most studies to date have focussed on simulation of the neutral ABL, where buoyancy effects have mostly been ignored or modelled using a Boussinesq type approach (Alinot and Masson 2005). Furthermore, if the computational domain is large enough, full variation of density as a function of pressure and temperature needs to be introduced, leading to significant computational challenges.

The concepts of atmospheric stability are above all applicable to the natural environment found in desert ecosystems. The lack of moisture and clouds leads to conditions that are particularly amenable to the formation of thermal stratifications. During the day solar radiation heats the ground to significantly high temperatures, resulting in a superadiabatic layer close to the ground and unstable atmospheric conditions, while the clear skies at night lead to thermal radiative heat loss and the formation of temperature inversions and stable atmospheric conditions. One would therefore expect to find consistent diurnal variation between unstable and stable conditions. A description of the wind in such areas would therefore be incomplete without considering Monin-Obukhov similarity theory, and these areas also provide the opportunity to measure wind and temperature profiles under the influence of heat transfer.

What is more, the implications of atmospheric flow in arid regions are far reaching. It represents the most significant factor in shaping the ecosystems found there. This includes seed transport and deposition as well as sand dynamics, including the formation and erosion of dunes. These factors shape the entire ecosystem including all trophic levels and food webs. It is therefore very important to have an accurate description of the atmospheric flow in such regions. It is also likely, according to Thomas et al. (2005) that the dune fields of southern Africa will experience significant reactivations as a consequence of twenty-first century climate change, which is likely to hasten the process of desertification. Understanding the effects of wind flow over desert sand dunes is therefore very important.

Up until now though, no one has investigated the effect of the thermally influenced atmospheric boundary layer on the flow over a sand dune (Livingstone et al. 2007). As sand dunes typically occur in desert environments where there is 
substantial diurnal variation in the structure of the atmospheric boundary layer due to heating and cooling of the surface, it seems sensible for this effect to be clarified. As wind tunnels typically can only reproduce the neutral ABL (Franke et al. 2011), and field studies are typically limited in scope (Livingstone et al. 2007), CFD seems to be the obvious route to quantify these effects, which could make a new contribution to understanding the behaviour of the flow fields over desert sand dunes.

Thus, the focus of the work conducted in this study entailed firstly the empirical measurement of wind speed and temperature at different vertical heights in the interdune area of the Namib Desert, using a wind mast. The data were analysed and the various stability regimes identified. This included the determination of aerodynamic surface roughness, ground heat flux and stability parameters. These factors were used to describe the typical conditions that occurred in the area during the time of measurement and determine continuous profiles of velocity and temperature that were commensurate with the measured data.

These velocity and temperature profiles were used to inform a description of the inlet boundary conditions of the subsequent CFD simulations, using STAR$\mathrm{CCM}+$ (CD-adapco Inc. 2011). The first requirement was to find model parameters that would allow for the maintenance of the inlet profiles over an extensive downwind fetch of the numerical domain, therefore producing profiles that were self-maintaining and horizontally homogenous. Different turbulence models were tested for their capability to achieve this.

Subsequently the flow over a cosine hill was simulated using these model parameters to investigate the accuracy of various turbulence models in the predictions of wake effects and separation. With this determined, it was possible to model the flow over a full scale two-dimensional transverse dune under the various atmospheric stability conditions.

\subsection{Project Objectives and Outline}

The scope of this study can therefore be broken down into the following objectives:

- The measurement of velocity and temperature profiles that capture the diurnal variation in ground heat flux and therefore the different $A B L$ stability classes.

- The assessment of these measurements to determine the parameters that describe the velocity and temperature profiles according to the stability condition of the atmosphere, and the surface conditions.

- The extrapolation of these profiles using Monin-Obukhov similarity theory. 
- Based on these parameters, the ABL under different thermal stability conditions can be simulated using CFD, the commercial code STAR$\mathrm{CCM}+$ will be used throughout this study.

- The CFD code must demonstrate the capability of maintaining the profiles of velocity, temperature and the turbulence quantities throughout the computational domain, i.e. horizontal homogeneity of the profiles must be demonstrated in an empty domain.

- The effects of gravity and buoyancy must be accounted for in the code.

- Full variation in temperature, pressure and density through the ideal gas law must be allowed so that the accurate behaviour of the atmosphere under the influence of gravity can be captured.

- The CFD solution must be numerically stable.

- Different turbulence models must be tested to assess their performance in achieving the above goals.

- The performance of the turbulence models thus modified must be validated for flow in separated regions: the flow over a cosine hill can serve this purpose.

- Once maintenance of the stipulated profiles has been achieved and the performance of the models for separated flow has been validated, the flow over an idealised two-dimensional transverse dune can be investigated, under the different thermal stratifications.

The thesis thus presented will have the following structure:

- Chapter 2 is composed of a literature review of the ABL and an account of the Monin-Obukhov similarity theory that describes the different stability classes of the thermally stratified atmosphere.

- Chapter 3 reviews the literature surrounding the application of CFD to model the ABL, including its application to the modelling of flow involving aeolian geomorphology.

- Chapter 4 describes the methods and results for the empirical determination of the wind speed and temperature profiles measured in a desert environment where diurnal variation leads to the lower atmosphere in these areas cycling through the different thermal stability states.

- Chapter 5 looks at the implementation of the Monin-Obukhov theory with CFD codes to account for the atmospheric boundary layer under different thermal stability conditions and buoyancy, where the vertical profiles of velocity, temperature and turbulence quantities must be maintained along the horizontal length of the fluid domain.

- Chapter 6 investigates the performance of different turbulence models, modified to be able to account for buoyancy and thermal stratification, to accurately simulate separation in the wake of the flow over a cosine hill.

- Chapter 7 applies these same CFD models to the flow over an idealised transverse dune immersed in an ABL with different thermal stratifications.

- Chapter 8 draws the final conclusions derived from the preceding work. 


\section{A REVIEW OF ABL THEORY IN LITERATURE}

The atmosphere consists mainly of oxygen and nitrogen but it also contains small amounts of other gases such as water vapour, carbon dioxide, hydrogen and helium, as well as the rare inert gases (argon, krypton, neon etc.). It may, however, be regarded as a homogenous gas of uniform composition (Kröger 2004). If one considers a parcel of air lifted upward in the atmosphere, one expects its pressure to decrease in response to the atmospheric pressure field, under the influence of gravity. This will lead to a decrease in the temperature if there is no heat transferred to it by either conduction or radiation, in other words if the process is adiabatic, due to the expansion of the parcel. Vertical turbulent motions in the ABL are rapid enough to justify the adiabatic assumption for such motions in the atmosphere (Blackadar 1997, Arya 2001c, Kröger 2004).

Following the procedure of Kröger (2004) and Arya (2001c), the pressure gradient is given by the hydrostatic equation:

$$
\partial P / \partial z=-\rho g
$$

where $g$ is the gravitational acceleration and $\rho$ is the density. Gravity is usually a function of both latitude and altitude but changes are considered small enough to be negligible for the purposes of this analysis and a constant value of $9.81 \mathrm{~m} / \mathrm{s}^{2}$ is used throughout (Kröger 2004). For an isentropic process:

$$
P / \rho^{\gamma}=\text { constant }
$$

where $\gamma$ is the heat capacity ratio and for dry air:

$$
\gamma=c_{p} / c_{v}=1.4
$$

Another fundamental relationship is given by the ideal gas law:

$$
\rho=P / R T
$$

$R$, the specific gas constant has a value of $287.08 \mathrm{~J} / \mathrm{kgK}$ for dry air and is related to the heat capacities by:

$$
c_{p}-c_{v}=R
$$

By substituting Equation 2-4 into Equation 2-2 and differentiating with respect to altitude we obtain:

$$
\frac{(1-\gamma)}{\gamma P} \frac{\partial P}{\partial z}+\frac{1}{T} \frac{\partial T}{\partial z}=0
$$

Combining Equations 2-1, 2-3 and 2-6 we find the temperature gradient: 


$$
\frac{\partial T}{\partial z}=-\frac{g(\gamma-1)}{\gamma R}=-\frac{g}{c_{p}}=-\Gamma=-0.009775 \mathrm{~K} / \mathrm{m}
$$

This temperature gradient is known as the dry adiabatic lapse rate $(\Gamma)$. Integration of the above equation yields:

$$
T=T_{0}-\frac{g(\gamma-1)}{\gamma R} z=T_{0}-\Gamma z
$$

where $T_{0}$ is the temperature at ground level. From Equations 2-1 and 2-4 we have:

$$
\partial P / P=-g \partial z / R T
$$

Substituting Equation 2-8 into Equation 2-9 and integrating yields:

$$
P=P_{0}\left[1-\frac{g(\gamma-1)}{\gamma R T_{0}} z\right]^{\gamma /(\gamma-1)}=P_{0}\left(1-\frac{\Gamma}{T_{0}} z\right)^{3.5}
$$

where $P_{0}$ is the reference pressure at ground level. Now rearranging Equation 2-6 gives:

$$
\partial T / T=\left(R / c_{p}\right)(\partial P / P)
$$

Integration of which gives the Poisson equation:

$$
T=T_{0}\left(P / P_{0}\right)^{k}
$$

where the exponent:

$$
k=R / c_{p} \cong 0.286
$$

Equation 2-12 is used in the definition of potential temperature $\theta$, that is, the temperature a parcel of air will attain if it were brought adiabatically to the standard pressure of the earth's surface (Arya 2001c, Kröger 2004, Arya 2005), and is related to actual temperature $T$ by:

$$
\theta=T\left(P_{0} / P\right)^{k}
$$

Potential temperature has the convenient property of being conserved with height and does not change during vertical movements of an air parcel in the adiabatic atmosphere. For a non-adiabatic atmosphere, from 2-14:

$$
\frac{\partial \theta}{\partial z}=\frac{\theta}{T}\left(\frac{\partial T}{\partial z}+\Gamma\right) \cong \frac{\partial T}{\partial z}+\Gamma
$$


The above relationship can be used to express the difference in the potential temperatures between any two height levels as:

$$
\Delta \theta=\Delta T+\Gamma \Delta z
$$

and the integral version of the same relationship as:

$$
\theta-\theta_{0}=T-T_{0}+\Gamma z
$$

However, at the earth's surface it is often true that $\theta_{0}=T_{0}$ and therefore:

$$
\theta=T+\Gamma z
$$

The advantage of using Equations 2-17 and 2-18 for calculating potential temperature in the $\mathrm{ABL}$ is that the measurement or estimate of the pressure at each height level is not required.

These results further allow us to define the concepts of static stability. A parcel of air that moves up or down will heat up or cool down according to the adiabatic lapse rate, and therefore often find itself in an environment where its density differs from that of the surrounding air, due to the environment being under the influence of heat flux at the surface. In the presence of gravity this density difference will result in a buoyant force being applied to the air parcel, which can either accelerate or decelerate its vertical movement. In the event that the vertical movement of the parcel is enhanced and it is moved further away from its equilibrium position by the buoyant force, the environment is called statically unstable. If the parcel is decelerated and is moved back to its equilibrium position, the atmosphere is called stable or stably stratified. If on the other hand there is no buoyancy force on the parcel, i.e., if the environmental lapse rate is equal to the adiabatic lapse rate and the density on the parcel and the environment is the same after the parcel is displaced, the atmosphere is considered neutral. For neutral conditions then, the surface heat flux is zero and the turbulent motion leads to the emergence of the adiabatic lapse rate temperature profile. The categories defined in terms of the atmospheric lapse rate are as follows (Azad 1993, Arya 2001c):

- Unstable, when $\partial T / \partial z<-\Gamma$, or $\partial \theta / \partial z<0$

- Neutral, when $\partial T / \partial z=-\Gamma$, or $\partial \theta / \partial z=0$

- Stable, when $\partial T / \partial z>-\Gamma$, or $\partial \theta / \partial z>0$

On the basis of environmental lapse rate (LR) relative to the adiabatic lapse rate, atmospheric layers are categorised as follows, shown graphically in Figure 2-1 (Arya 2001c):

- Superadiabatic, when LR > $\Gamma$

- Adiabatic, when LR $=\Gamma$

- Subadiabatic, when $0<\mathrm{LR}<\Gamma$

- Isothermal, when $\partial T / \partial \mathrm{z}=0$

- Inversion, when $\partial T / \partial \mathrm{z}>0$ 
The results derived above are based on the assumption of dry air. If the air contains significant amounts of water vapour, different results are obtained (Kröger 2004). If a parcel of moist air rises in a gravitational field, adiabatic cooling will take place and the air will reach the point of saturation. If further rising takes place the cooling will cause the water vapour to condense and precipitate. The condensation process will release energy from the vapour and this will be taken up by the surrounding air in a pseudo-adiabatic process (Kröger 2004). Modifications to all the relations described above to account for moisture in the air can be found in various texts (Lumley 1964, Azad 1993, Arya 2001c, Kröger 2004, Arya 2005,); however, the dry air assumption will be made for the work conducted in this study. Furthermore, the effects that the Earth's rotation, through the Coriolis force, has on the momentum equation will also be ignored and the Navier-Stokes equations as derived for a nonrotating system will suffice (Blackadar 1997, Arya 2001a).

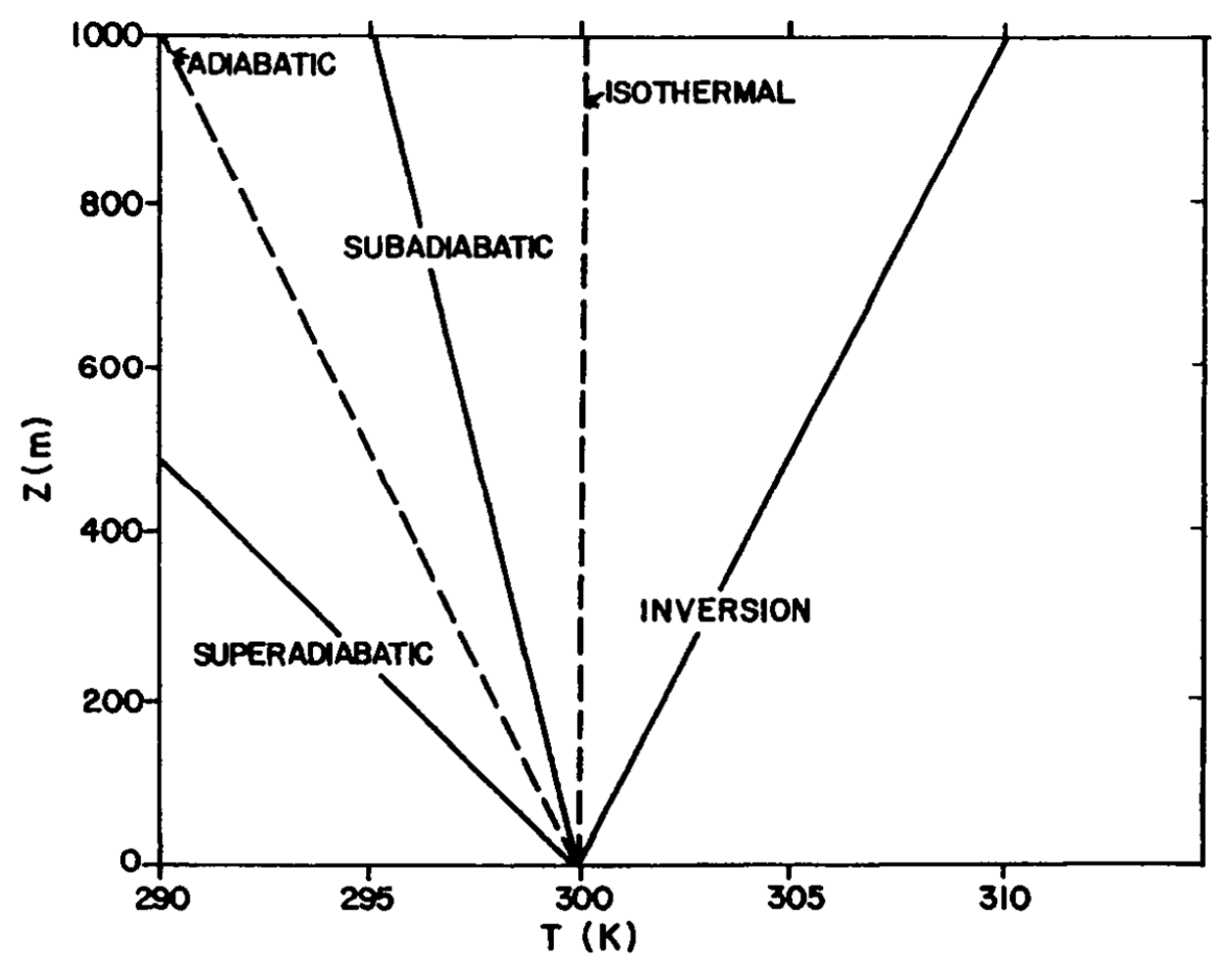

Figure 2-1: Schematic of stability categories on the basis of temperature gradient, taken from Arya (2001c)

It is now necessary to consider the effect of turbulence on the dispersion of various properties that the ABL accumulates at the surface, such as momentum and heat. In other words we need to describe the vertical flux of these properties as fluid parcels are transported up and down within the ABL by turbulent eddies. The next arguments are formulated for the lower $10 \%$ of the ABL, known as the surface layer where most of the exchanges of energy and moisture take place. It usually exhibits little change with height in the vertical fluxes of momentum, heat and water vapour (Blackadar 1997, Arya 2005). 
Furthermore we will initially consider only the neutral surface layer (zero heat flux) over a flat and uniform surface where the momentum flux may be considered constant, and the Coriolis force is ignored. A simple similarity hypothesis can be formulated where the mean wind shear, $\partial u / \partial z$, is dependent only on the height $z$ above the ground, the surface shear stress $\left(\tau_{o}\right)$ and the fluid density (Arya 2001a). The boundary layer is furthermore assumed to be fully turbulent and to have no streamwise gradients, i.e., to be horizontally homogenous. Thus:

$$
\partial u / \partial z=f\left(z, \tau_{o}, \rho\right)
$$

The shear stress, derived from exchange theory, is given by:

$$
\tau=\rho K_{m} \partial u / \partial z
$$

where $K_{m}$ is the kinematic turbulent or eddy viscosity and is a function of height, while the molecular viscosity is small in comparison to the eddy viscosity and can be ignored (Blackadar 1997). The eddy viscosity has dimensions of velocity multiplied by length leading to:

$$
K_{m}=\kappa u_{*} Z
$$

where $u_{*}$ is the friction velocity which is independent of height. The constant of proportionality $\kappa$ is the von Karman constant with an experimentally determined value of between 0.38 and 0.45 (Lumley 1964, Azad 1993, Blackadar 1997). It can be seen that shear stress is constant in the neutral surface layer and:

$$
\tau=\tau_{0}=\rho u_{*}^{2}
$$

Dimensional analysis now yields the dimensionless wind shear:

$$
\varphi_{m}=\frac{\kappa z}{u_{*}} \frac{\partial u}{\partial z}
$$

This quantity is a function of heat flux but in the neutral layer it has a value of one (Blackadar 1997). Integration of Equation 2-23 with respect to $z$ gives the neutral logarithmic velocity profile law:

$$
u=\frac{u_{*}}{\kappa} \ln \left(\frac{Z}{z_{0}}\right)
$$

Here $z_{0}$ has been introduced as a dimensional constant of integration and can be seen as the height at which the wind speed goes to zero. This height or length is commonly referred to as the aerodynamic roughness length or roughness parameter and is indicative of the surface roughness (Wiernga 1993, Blackadar 1997, Arya 2001a). 
The above argument for neutral layers can be extended to account for layers that are heated or cooled from below, as neutral layers are the exception rather that the rule in the lower atmosphere (Arya 2001b). This includes a derivation of the vertical temperature distribution, which is equal to the adiabatic lapse rate in neutral layers. The Monin-Obukhov similarity theory provides the most suitable framework for describing the atmospheric conditions under the influence of heat transfer (Arya 2005).

The similarity hypothesis again assumes a horizontally homogenous layer where the turbulent fluxes of momentum and heat are independent of height and therefore constant, and that the molecular exchanges are insignificant compared to the turbulent exchanges (Arya 2001b). Rotational effects are again ignored. The mean flow and turbulence characteristics now depend only on four independent variables: the height above the surface $z$, the surface drag $\tau_{0} / \rho$, the surface heat flux $\dot{q}_{0} / \rho c_{p}$, and the buoyancy variable $g / T_{0}$. These four independent variables have three fundamental dimensions (time, length and temperature) which according to Buckingham's $\Pi$-theorem will yield only one dimensional combination. The combination usually chosen is the buoyancy or stability parameter:

$$
\zeta=z / L
$$

where $L$ is the Monin-Obukhov length and is defined as:

$$
L=\frac{u_{*}^{2} T_{0}}{\kappa g \theta_{*}}
$$

with $\theta_{*}$, the scaling temperature, defined as:

$$
\theta_{*}=\frac{-\dot{q}_{0}}{\rho c_{p} u_{*}}
$$

From the definition it is clear that $L$ may range from $-\infty$ to $\infty$, with the extreme values occurring when the heat flux approaches zero from the positive (unstable) and the negative (stable) side (Arya 2001b). Depending on the direction of the heat flux the atmospheric stability may be defined in terms of the Monin-Obukhov length as:

- Unstable, when $L<0$

- Neutral, when $L=\infty$

- Stable, when $L>0$

The magnitude $|L|$ represents the height at which the magnitudes of mechanical and buoyant production of turbulence is equal, or stated in another way, the thickness of the layer near the surface in which the shear of friction effects are 
important (Arya 2001b, Kröger 2004). Close to the surface $(z \ll|L|)$ turbulent production due to wind shear tends to dominate while the effects of buoyancy remain insignificant. As one moves away from the surface and $z \gg|L|$, turbulent production due to buoyancy starts to dominate over shear generated turbulence. It is therefore expected that profiles close to the ground will be approximately logarithmic as it has been shown that this kind of profile is characteristic of conditions where mechanical production is dominant, i.e. neutral conditions (Blackadar 1997). The ratio of buoyancy produced turbulence to mechanically produced turbulence leads to the Richardson number defined as:

$$
R i=\frac{g}{\theta} \frac{\partial \theta / \partial z}{(\partial u / \partial z)^{2}}
$$

Due to the definition of stability through the potential temperature gradient it can be seen that, similar to the Monin-Obukhov length, Richardson number is related to the stability of the atmosphere as:

- Unstable, when $R i<0$

- Neutral, when $R i=\infty$

- Stable, when $R i>0$

By this definition, under stable conditions the buoyant production of turbulence is negative, and thus turbulence must do work against gravity, which consumes turbulent kinetic energy. As the mechanical production is the only source of turbulence under these conditions, if it is insufficient to replace the losses the turbulence will die out. This leads to the definition of the critical Richardson number where if $R i>R i_{c}$ the turbulence will be completely suppressed. Observations and theory have shown that this value is about 0.2 (Blackadar 1997).

The similarity prediction that follows from the Monin-Obukhov similarity hypothesis is that any mean flow or turbulence quantity in the surface layer, when normalised by an appropriate scaling parameter, must be a unique function of $z / L$ only (Arya 2001b, Arya 2005). The scaling parameters are the length scales $z$ and $L$, the velocity scale $u_{*}$ and the temperature scale $\theta_{*}$. Any number of similarity relations can therefore be written for a dependent variable of interest. The dimensionless wind shear and potential temperature gradient can be expressed as:

$$
\begin{gathered}
\frac{\kappa z}{u_{*}}\left(\frac{\partial u}{\partial z}\right)=\varphi_{m}(\zeta) \\
\frac{\kappa z}{\theta_{*}}\left(\frac{\partial \theta}{\partial z}\right)=\varphi_{h}(\zeta)
\end{gathered}
$$


Where $\varphi_{m}$ and $\varphi_{h}$ are the universal similarity functions that relate the constant fluxes;

$$
\begin{gathered}
\tau=\tau_{0}=\rho u_{*}^{2} \\
\dot{q}=\dot{q}_{0}=-\rho c_{p} u_{*} \theta_{*}
\end{gathered}
$$

to the mean gradients in the surface layer (Arya 2001b). In addition, from exchange theory it can be seen that the dynamic turbulent viscosity $\mu_{t}$ is related to the similarity functions by:

$$
\mu_{t}=\rho K_{m}=\frac{\rho \kappa u_{*} Z}{\varphi_{m}\left(\frac{Z}{L}\right)}
$$

It is furthermore easy to show that the Richardson number is related to the stability parameter $\zeta$ by:

$$
R i=\zeta \frac{\varphi_{h}(\zeta)}{\varphi_{m}(\zeta)^{2}}
$$

Therefore, from measured gradients of velocity and temperature the Richardson number can be calculated and from that the stability parameter can be determined, provided that the forms of the similarity functions are known. The similarity functions must be determined empirically by careful experimentation and the equations that will be employed in this study are those derived by Businger et al. (1971) and Dyer (1974):

$$
\begin{array}{ll}
\varphi_{h}=\varphi_{m}^{2}=(1-16 z / L)^{-\frac{1}{2}}, & \mathrm{~L}<0 \\
\varphi_{h}=\varphi_{m}=1+5 z / L, & \mathrm{~L}>0
\end{array}
$$

These can then be related to the Richardson number:

$$
\begin{array}{ll}
\zeta=R i, & R i<0 \\
\zeta=R i /(1-5 R i), & 0 \leq R i \leq 0.2
\end{array}
$$

The vertical wind and temperature profiles can now be determined by integration of Equations 2-29 and 2-30. This yields the following relations for wind velocity and potential temperature with respect to height:

$$
u(z)=\left(\frac{u_{*}}{\kappa}\right)\left[\ln \left(\frac{Z}{z_{0}}\right)-\psi_{m}\left(\frac{Z}{L}\right)\right]
$$




$$
\theta(z)-\theta_{0}=\left(\frac{\theta_{*}}{\kappa}\right)\left[\ln \left(\frac{Z}{z_{0}}\right)-\psi_{h}\left(\frac{z}{L}\right)\right]
$$

where $\psi_{m}$ and $\psi_{h}$ are the integrated forms of the similarity functions related to them respectively as:

$$
\begin{aligned}
& \psi_{m}\left(\frac{z}{L}\right)=\int_{z_{0} / L}^{z / L}\left[1-\varphi_{m}(\zeta)\right] \frac{d \zeta}{\zeta} \\
& \psi_{h}\left(\frac{z}{L}\right)=\int_{z_{0} / L}^{z / L}\left[1-\varphi_{h}(\zeta)\right] \frac{d \zeta}{\zeta}
\end{aligned}
$$

The values of $z_{0} / L$ are usually quite small and can be replaced by zero. With this approximation $\psi_{m}$ and $\psi_{h}$ can be determined for any appropriate form of $\varphi$, so that for Equations 2-35 and 2-36 we obtain:

$$
\begin{array}{ll}
\psi_{h}=\psi_{m}=-5 \frac{Z}{L}, & L>0 \\
\psi_{m}=\ln \left[\left(\frac{1+x^{2}}{2}\right)\left(\frac{1+x}{2}\right)^{2}\right]-2 \tan ^{-1} x+\frac{\pi}{2}, & L<0 \\
\psi_{h}=2 \ln \left(\frac{1+x^{2}}{2}\right), & L<0
\end{array}
$$

where:

$$
x=(1-16 z / L)^{1 / 4}
$$

It can be noted that the profiles will start to deviate from the log law with increasing values of $z / L$. Under stable conditions the profiles tend to become linear for large values of $z / L$ and under unstable conditions $\psi_{m}$ and $\psi_{h}$ are positive so that the profiles of velocity and temperature will become more curvilinear. The relations obtained from Monin-Obukhov similarity can be used to describe the boundary conditions of CFD simulations wishing to accurately model the atmospheric boundary layer under different thermal stability conditions. 


\section{A REVIEW OF THE CFD MODELLING OF THE TURBULENT ABL}

Armed with the insights garnered from the Monin-Obukhov similarity theory, attention can now be turned to the computational fluid dynamic (CFD) simulation of the ABL. CFD is a tool which is increasingly being used to study a wide variety of processes in the ABL, inversions or aerosol transport being examples. As already mentioned, these numerical simulations can be performed by using either the RANS equations or by large-eddy simulations, with LES being more accurate but significantly more computationally expensive. For this reason, the standard $k-\varepsilon$ turbulence model, widely employed in the simulation of the ABL due to the availability of appropriate boundary conditions and meteorological data (Blocken et al. 2007b, Hargreaves and Wright 2007, Franke et al. 2011, Blocken et al. 2011), will serve as the starting point in investigating of the ABL under the influence of surface heat flux.

The steady RANS equations use the time average, and lead to a statistically steady description of turbulent flow which eliminates the time dimension from the governing equations. The effect of the transient turbulent fluctuation on the mean flow field for the steady formulation has to be modelled and this is achieved by the addition of the Reynolds or turbulent stress tensor to the momentum equation, through the eddy viscosity, and the modification of the thermal conductivity in the energy equation, through the eddy diffusivity (Versteeg and Malalasekera 2007). These governing equations are given by the equations for continuity, momentum and energy. They are described in tensor form respectively by:

$$
\begin{gathered}
\int_{A} \rho u_{i} n_{i} d A=0 \\
\int_{A} \rho u_{j} u_{i} n_{i} d A=-\int_{A} P \delta_{i j} n_{i} d A+\int_{A} \tau_{i j} n_{i} d A+\int_{v} \rho g_{i} d V \\
\int_{A} \rho c_{p} T u_{i} n_{i} d A=\int_{V} \rho u_{i} g_{i} d V+\int_{A}\left[u_{j} \tau_{i j}+\frac{c_{p} \mu_{t}}{\sigma_{T}}\left(\frac{\partial T}{\partial x_{i}}\right)\right] n_{i} d A
\end{gathered}
$$

here $A$ is the outer surface area of a fixed volume $V, u_{i}$ is the velocity component along the $x_{i}$ direction, $n_{i}$ is the cosine director of the outward unit vector $\mathbf{n}$ perpendicular to the control surface $d A, \delta_{i j}$ is the Kronecker delta, $\tau_{i j}$ is the viscous stress tensor which includes the Reynolds stress tensor, $g_{i}$ is the gravitational vector component along $x_{i}$, and $\sigma_{T}$ is the turbulent Prandtl number for energy (Alinot and Masson 2005, Pontiggia et al. 2009).

Many ABL studies using CFD have been conducted, but these often assume equilibrium or neutral conditions (Blocken et al. 2007a, b, Hargreaves and Wright 
2007, Yang et al. 2008, Liu et al. 2011, Parente et al. 2011b). According to Blocken et al. (2007b), improper ABL modelling can yield large errors in the numerical results and the selection of appropriate boundary conditions is very important in achieving horizontally homogenous ABL flow over uniformly rough terrain. This means that the vertical mean flow profiles should be maintained in the streamwise direction along the fetch of the flow, with minimal streamwise gradients. This can only occur when these profiles are in equilibrium with the surface fluxes, and with the governing equations employed by the CFD code.

Even in the simplified case of neutral conditions, the achievement of horizontally homogenous flows nonetheless proves challenging. A particular observation in these cases is considerable acceleration of the flow near the surface and an inability to maintain the turbulent kinetic energy profiles (Hargreaves and Wright 2007). One of the reasons given for these errors is in the inconsistency between the wall functions employed by the turbulence models and the profiles that describe the ABL.

CFD codes employing RANS turbulence generally model the flow under turbulent conditions near walls using a wall function. The roughness of these surfaces is often expressed in terms of the equivalent sand-grain roughness height $k_{s}$, as is the case for the commercial codes FLUENT (Fluent 2006) and STAR-CCM+ (CD-adapco Inc. 2011). For the consistent and accurate application of the law of the wall the dimensionless wall distance $z^{+}$(often referred to as $y^{+}$is literature) must be in the range of 30 up to about 500 (White 1991), placing a limit on the position of the first grid node from the wall, $z_{p}$. If the wall roughness is expressed by an equivalent sand-grain roughness, Blocken et al. (2007a, b) suggest that four requirements be met simultaneously for accurate $\mathrm{ABL}$ simulation:

- A sufficiently high mesh resolution in the vertical direction close to the ground surface.

- A horizontally homogenous approach flow.

- A distance $z_{p}$ from the centre point $p$ of the wall-adjacent cell to the wall (ground or bottom of the domain) that is larger than the physical roughness height $k_{s}$ of the terrain $\left(z_{p}>k_{s}\right)$.

- Knowing the relationship between the equivalent sand-grain roughness $k_{s}$ and the corresponding aerodynamic roughness length $z_{0}$.

The first requirement is important for all CFD studies by ensuring the validity of the near wall equation by adhering to the $z^{+}$requirements. The second requirement implies that the flow profiles prescribed at the inlet of the domain should remain free of streamwise gradients, and be in balance with the governing equations. The third requirement entails that it is not physically meaningful to have cells with centre points within the physical roughness height, which can lead to numerical instability. Finally, the fourth requirement states that empirical information about the ground roughness, incorporated into the simulation by the use of wall functions, should be related to the aerodynamic roughness length. 
However, it is generally impossible to satisfy all four requirements (Hargreaves and Wright 2007, Blocken et al. 2007b).

Some of the inconsistencies between the sand-grain based wall function and the logarithmic wind velocity profile can be rectified by expressing the roughness length $z_{0}$ as an equivalent sand-grain height. A generally accepted relation between roughness length and equivalent sand-grain roughness height is (Blocken et al. 2007b, Franke et al. 2011):

$$
k_{s}=30 z_{0}
$$

However, this often leads to very large computational cells and hence bad resolution, due to the requirement that $z_{p}>k_{s}$. The use of a smaller $k_{s}$ value than the one corresponding to the inlet profiles yields better horizontal resolution near the wall but can lead to substantial horizontal inhomogeneity of the inflow profiles. Some other relations for $z_{0}$ and the obstruction height $\left(h_{0}\right)$ have been proposed, including:

$$
z_{0} / h_{0}=0.15
$$

for various types of crops and grass-land (Arya 2001a). If values of $k_{s}$ higher than $z_{p}$ are specified, the code internally sets the value of $k_{s}$ equal to $z_{p}$ (Blocken $e t a l$. 2011), and therefore care should be taken that at the very least $z_{p}>z_{0}$, despite the $z^{+}$requirements, as the flow must be stationary below $z_{0}$.

Some attempts have been made to simulate the ABL under the conditions established by the Monin-Obukhov theory using RANS two-equation turbulence models (Huser et al. 1997, Alinot and Masson 2005, Pontiggia et al. 2009, Meissner et al. 2009). The minimum requirement of the turbulence model for thermally influenced ABL flows is that it should account for both shear and buoyancy produced turbulence.

The standard $k-\varepsilon$ turbulence model, first proposed by Launder and Spalding (1974) meets this requirement and has been widely deployed in ABL simulations; consequently there is a large availability of $k$ and $\varepsilon$ properties of the atmospheric boundary layer in meteorological data (Alinot and Masson 2005). The $k-\varepsilon$ turbulence model achieves closure for the flow variables by introducing two additional transport equations for the turbulent kinetic energy $(k)$ and turbulent kinetic energy dissipation rate $(\varepsilon)$, respectively:

$$
\int_{A} \rho k u_{i} n_{i} d A=\int_{A}\left(\mu+\frac{\mu_{t}}{\sigma_{k}}\right) \frac{\partial k}{\partial x_{i}} n_{i} d A+\int_{V}\left[G_{k}+G_{b}-\rho \varepsilon-Y_{m}\right] d V
$$




$$
\begin{gathered}
\left.\int_{A} \rho \varepsilon u_{i} n_{i} d A=\int_{A}\left(\mu+\frac{\mu_{t}}{\sigma_{\varepsilon}}\right) \frac{\partial \varepsilon}{\partial x_{i}} n_{i} d A+\int_{V} C_{\varepsilon 1} \frac{\varepsilon}{k}\left(G_{k}+C_{\varepsilon 3} G_{b}\right)-C_{\varepsilon 2} \rho \frac{\varepsilon^{2}}{k}\right] d V \\
G_{k}=\tau_{i j} \frac{\partial u_{i}}{\partial x_{j}}=-\rho \overline{u_{i}^{\prime} u_{j}^{\prime}} \frac{\partial u_{i}}{\partial x_{j}}=\mu_{t}\left(\frac{\partial u_{i}}{\partial x_{j}}+\frac{\partial u_{j}}{\partial x_{i}}\right)-\frac{2}{3} \rho k \delta_{i j} \\
G_{b}=\beta g_{i} \frac{\mu_{t}}{\sigma_{T}} \frac{\partial T}{\partial x_{i}} \\
\mu_{t}=\rho C_{\mu} \frac{k^{2}}{\varepsilon}
\end{gathered}
$$

Here $\mu$ is the molecular dynamic viscosity and $\mu_{t}$ is the dynamic eddy viscosity, $G_{k}$ is the turbulent kinetic energy production due to shear, $G_{b}$ is the turbulent kinetic energy production due to buoyancy, $Y_{m}$ is the compressibility related kinetic energy production, and $\beta$ is the thermal volumetric expansion coefficient (Alinot and Masson 2005, Pontiggia et al. 2009). For an ideal gas the volumetric expansion coefficient is given by:

$$
\beta=-\frac{1}{\rho} \frac{\partial \rho}{\partial T}=\frac{1}{T}
$$

Further, $C_{\varepsilon 1}, C_{\varepsilon 2}, C_{\varepsilon 3}, \sigma_{k}, \sigma_{\varepsilon}$, and $C_{\mu}$ are empirical constants originally determined by Launder and Spalding (1974) and their values are given in Table 3-1.

Table 3-1: Original $k-\varepsilon$ model constants

\begin{tabular}{cccccc}
\hline$C_{\varepsilon 1}$ & $C_{\varepsilon 2}$ & $C_{\varepsilon 3}$ & $\sigma_{k}$ & $\sigma_{\varepsilon}$ & $C_{\mu}$ \\
\hline 1.44 & 1.92 & 0 & 1.0 & 1.3 & 0.09 \\
\hline
\end{tabular}

Due to the homogeneity requirements, the formulae used to specify the boundary conditions of the turbulence quantities in the atmospheric boundary layer must be in balance with the transport equations solved by the CFD code. The most widely used relations are those first proposed by Richards and Hoxey (1993) for the neutral atmospheric boundary layer, where they assumed constant properties in the direction of the flow with only variation in the vertical direction. Furthermore they assumed that pressure was constant in the flow direction and that the flow is driven by a shear stress applied at the top of the layer, which is constant throughout and given by Equation 2-22. Given these assumptions, the logarithmic 
form of the velocity profile given by Equation 2-24, and using Equations 3-6 to 3-10, they found:

$$
\begin{gathered}
k=\frac{u_{*}^{2}}{\sqrt{C_{\mu}}} \\
\varepsilon(z)=\frac{u_{*}^{3}}{\kappa z}
\end{gathered}
$$

These are very popular boundary conditions for RANS solutions to the neutral boundary layer (Franke et al. 2011). A similar approach can be used to find the appropriate boundary conditions for the Monin-Obukhov similarity theory. Again the assumption is made that the flow properties only vary in the vertical direction, the velocity is given by Equation 2-39 and the turbulent viscosity is given by Equation 2-33. Based on measurements of turbulent kinetic energy budget terms in the surface layer over flat terrain (Alinot and Masson 2005), one can find:

$$
\varepsilon(z)=\frac{u_{*}^{3}}{\kappa z} \varphi_{\varepsilon}\left(\frac{Z}{L}\right)
$$

where

$$
\varphi_{\varepsilon}\left(\frac{Z}{L}\right)= \begin{cases}1-\frac{z}{L} & L<0 \\ \varphi_{m}\left(\frac{Z}{L}\right)-\frac{z}{L} & L>0\end{cases}
$$

Solving the $k-\varepsilon$ equations yields the profile for $k$ for thermally stratified atmospheric boundary layers (Alinot and Masson 2005, Pontiggia et al. 2009):

$$
k(z)=\sqrt{\frac{\mu_{t} \varepsilon}{\rho C_{\mu}}}=5.48 u_{*}^{2} \sqrt{\frac{\varphi_{\varepsilon}\left(\frac{Z}{L}\right)}{\varphi_{m}\left(\frac{Z}{L}\right)}}
$$

where the constant 5.48 has been empirically determined for the neutral atmospheric boundary layer (Alinot and Masson 2005). To ensure that the profiles derived for velocity, temperature and turbulence properties from the MoninObukhov similarity theory are exact solutions to the $k-\varepsilon$ model, the values of the model constants $C_{\mu}, C_{\varepsilon 1}$, and $C_{\varepsilon 3}$ must be changed. From Equation 3-16, by combining Equation 2-33 and Equation 3-14 it is clear that the value of $C_{\mu}$ must be: 


$$
C_{\mu}=5.48^{-2}
$$

while an expression for $C_{\varepsilon 1}$ can be obtained from the transport equation of $\varepsilon$ (Equation 3-7), by introducing the empirical expressions of the Monin-Obukhov theory for neutral conditions (Alinot and Masson 2005):

$$
C_{\varepsilon 1}=C_{\varepsilon 2}-\frac{\kappa^{2}}{\sigma_{\varepsilon} \sqrt{C_{\mu}}}=1.176
$$

Furthermore, many values of $C_{\varepsilon 3}$ have been suggested in the literature (Kitada 1987, Alinot and Masson 2005, CD-adapco Inc. 2011) and they range from -0.8 for unstable conditions to 2.15 for stable conditions.

To account for buoyancy forces most studies apply the Boussinesq approximation where the density is taken to be constant (i.e. the flow is incompressible) and is assumed to vary linearly with temperature only in the gravity term of the momentum equation, Equation 3-2 (Kitada 1987, Alinot and Masson 2002, Alinot and Masson 2005, Pontiggia et al. 2009, Meissner et al. 2009). An alternative is to assume a weakly compressible fluid where the density is calculated using constant pressure as this leads to a faster and more stable numerical solution, and allows for the temperature profile to be more easily maintained than for the compressible situation (Huser et al. 1997, Pontiggia et al. 2009).

It is also true that simulations often show convergence problems when temperature profiles are included (Meissner et al. 2009), and stable atmospheric conditions are much easier to handle than unstable stratification, leading to many studies considering only neutral and stable conditions (Huser et al. 1997, Pontiggia et al. 2009). However, it is preferable to have a CFD model that allows for the full variation of density temperature and pressure as occurs in the real ABL, while still providing good maintenance of the vertical flow profiles through the length of the domain (i.e.: displays horizontal homogeneity). In this way buoyancy can be accounted for by adding weight to the fluid, through full variable density in a pressure field determined by gravitational force (hydrostatic pressure), while both of these can be coupled to temperature through the ideal gas law. The establishment of a numerically stable CFD model of the ABL that displays the characteristics of full compressibility and thermal stratification effects, with buoyancy forces accounted for by the fluid weight is a considerable challenge.

The biggest problem with maintaining the temperature profile in a CFD code is that even in the neutral atmosphere there is a temperature gradient (adiabatic lapse rate) with height over the computational element. The current form of the CFD transport equations, given by Equations 3-1 to 3-3 and Equations 3-6 to 3-10, assumes that temperature is conserved with height under conditions of zero heat transfer. Therefore, even under neutral atmospheric conditions, the CFD code 
activates the heat conduction terms as it sees a vertical temperature gradient over the computational element. A correction heat source must therefore be added to the energy equation (Equation 3-3) (Kristóf et al. 2009). We have seen that potential temperature has the property of being conserved with height (thus its use in exchange theory for heat (Blackadar 1997)), and therefore we can transform the temperature gradient in the energy equation to potential temperature gradient, so that zero heat transfer will prevail under neutral conditions. The same is true for the buoyant production of turbulence, which should be zero under neutral conditions, and therefore this term in the turbulent transport equations must also be transformed to potential temperature gradient (Kristóf et al. 2009).

The choice of solver and boundary conditions is also critical if the correct pressure, density and temperature profiles are to be achieved. It is noted that for a horizontally homogenous boundary layer the pressure cannot change along the horizontal length of the domain. As pressure differences are usually the driving force behind the flow field in any CFD simulation, the boundary conditions for the simulation of the ABL must be selected so that the shear stress can drive the flow, and the pressure can respond to the fluid weight without the model being over constrained. If these criteria can be met it should be possible to construct a CFD model that accounts for compressibility by the gravity force (and the resulting hydrostatic pressure field), the variation in temperature and density that this brings about, and to maintain these profiles along the fetch of the computational domain.

In addition, every turbulence model has its limitations. The standard $k$ - $\varepsilon$ model used to derive the above relations is no exception. It is for example known to under predict flow separation as a consequence of significantly over predicting the pressure recovery in the recirculation region (Kim and Boysan 1999). Modifications to the standard $k-\varepsilon$ model have been made to bring about improvements, such as the renormalisation group (RNG) $k-\varepsilon$ model which renormalizes the Navier-Stokes equations to account for small-scale turbulence (Versteeg and Malalasekera 2007); the realisable $k-\varepsilon$ model contains a new transport equation for the turbulent kinetic energy dissipation rate $(\varepsilon)$ and also, $C_{\mu}$ is expressed as a function of mean flow and turbulence properties rather than assumed to be constant (CD-adapco Inc. 2011).

Other two equation RANS models are also available, like the $k$ - $\omega$ model where the transport equations are the turbulent kinetic energy $(k)$ and the specific dissipation rate $(\omega)$. These models have been shown to perform much better than $k-\varepsilon$ models in adverse pressure gradients and therefore in predicting separation, but are very sensitive to free-stream/inlet conditions (Versteeg and Malalasekera 2007, CD-adapco Inc. 2011). The specific dissipation rate is related to the $k$ and $\varepsilon$ equations by:

$$
\omega=\varepsilon / C_{\mu} k
$$


A compromise between the advantages of the $k-\varepsilon$ and $k-\omega$ models is the shear stress transport (SST) $k$ - $\omega$ model which employs the $k$ - $\omega$ model near the surface and the $k-\varepsilon$ model in the free shear layers through the use of a blending function (CD-adapco Inc. 2011). Good performance of the SST $k$ - $\omega$ model for ABL flow around blunt bodies has been shown (Yang et al. 2008). The SST $k$ - $\omega$ model has also been adopted for detached eddy simulation (DES) turbulence models, which combine the features of RANS simulation in part of the flow and large eddy simulation (LES) in the separated regions. However, these models solve the unsteady transport equations and are still significantly more computationally expensive than the steady RANS models (CD-adapco Inc. 2011).

It is therefore important to assess the performance of different turbulence models in any application, with this one being no exception. The principles described above, which relates Monin-Obukhov similarity theory to the $k-\varepsilon$ turbulence model, can therefore be extended to other RANS turbulence models provided that they account for turbulence produced by gravity and buoyancy effects.

CFD has also been used in the description of aeolian geomorphology, specifically the wind and sand flow over dunes. It has proven to be a valuable tool in the study of the processes that shape desert sand dunes. There has furthermore been a recent proliferation of field and wind tunnel data concerning wind processes and it has become appropriate to apply new refinements to the CFD models of dune flow to provide new insights (Livingstone et al. 2007). Parsons et al. (2004a) modelled the flow over an idealised transverse dune under neutral conditions and found a deceleration of the flow immediately upwind of the dune followed by windward slope acceleration to a maximum velocity at the crest and subsequent flow reversal and separation in the lee. These results corresponded well to previous investigations (Wiggs et al. 1996, Parsons et al. 2004a, Livingstone et al. 2007). A major advantage of CFD in the study of flow over dunes lies in resolving the flow in the separation zone on the lee side (Livingstone et al. 2007), which is characterised by separation immediately in the lee due to an adverse pressure gradient and the formation of a shear zone expanding towards the point of reattachment and then dissipating (Parsons et al. 2004a, b).

Furthermore the effect of flow in three dimensions over dunes has also been investigated in various studies, which include wind tunnel tests and CFD (Liu et al. 2011, Joubert et al. 2012). Significant three-dimensional effects were found that would not be captured in two-dimensional simulations. Liu et al. (2011) compared two-dimensional and three-dimensional flow over an idealised transverse dune and found that the two-dimensional simplified model was sufficient to simulate a dune section. However, lateral inhomogeneity of flow in the leeward side of the three-dimensional dune model showed that it is not always correct to simplify the simulation of a sand dune into two-dimensions. The results between the zero plane three-dimensional model and the two-dimensional model were, however, qualitatively similar (Liu et al. 2011). 


\section{WIND DATA ACQUISITION AND ANALYSIS}

A wind mast was used to gather wind speed and temperature data at different vertical heights in the interdune area of the Namib Desert great sand sea. The wind profiles thus measured were analysed using Monin-Obukhov similarity theory so that the various stability classes could be identified. Descriptions of the surface conditions that led to the formation of the thermally stratified atmosphere were derived and continuous profiles for velocity and temperature for each set of measurements were produced.

\subsection{Wind Measurement}

The study area is on the northern edge of the Namib Sand Sea, east of Gobabeb Training and research Centre. The area is well known for its large complex linear dunes which are tens of kilometres in length, $500 \mathrm{~m}$ to $1000 \mathrm{~m}$ wide and up to $80 \mathrm{~m}$ high (Bristow et al. 2000). In between the linear dunes are broad gravel covered interdune areas of low relief, with some patches of grass, as shown in Figure 4-1.

Experimental determination of the surface conditions of homogenous terrain, such as an interdune, requires certain features when using wind masts, and can be summarised as follows (Wiernga 1993): firstly, observations must be made on a slender mast on booms which are much longer than the diameter of either the mast or the anemometer, with vertical spacing being sufficient to avoid interference; second, well calibrated anemometers must be used and the wind speed must be averaged over at least 10 minutes; third, temperature gradients are measured simultaneously for diabatic profile correction.

Wind measurement was achieved through the use of a $10 \mathrm{~m}$ high wind mast, shown in its position in the interdune in Figure 4-1. The mast consisted of four aluminium cup type anemometers and shielded thermocouples at different heights along the vertical length. The sensors were placed at heights of $2.5 \mathrm{~m}, 5.0 \mathrm{~m}$, $7.5 \mathrm{~m}$ and $10 \mathrm{~m}$ (corresponding to measurement heights $z_{1}, z_{2}, z_{3}$ and $z_{4}$ respectively) on horizontal arms, $0.5 \mathrm{~m}$ in length, to minimize the effect of interference of the mast on the measurements. A pair of wind speed and temperature sensors is shown in Figure 4-2 and the full mast used is shown in Figure 4-3. A wind vane was also included to record the wind direction. The time averaged wind speeds and temperatures were logged at intervals of 10 minutes to two data loggers. For further information on the data logging equipment, calibration and mast specifications the reader is referred to Joubert (2010), who ensured that the data transfer cables and logging equipment was resistant to the desert elements. Equipment information and sensor calibration parameters are given in Appendix H. The sensors were calibrated by the supplier and tested against calibrated sensors known to give accurate results, to ensure that consistent readings were recorded. The experiment was done in collaboration with other researchers looking at different aspects of the flow situation (Joubert et al. 2012). 


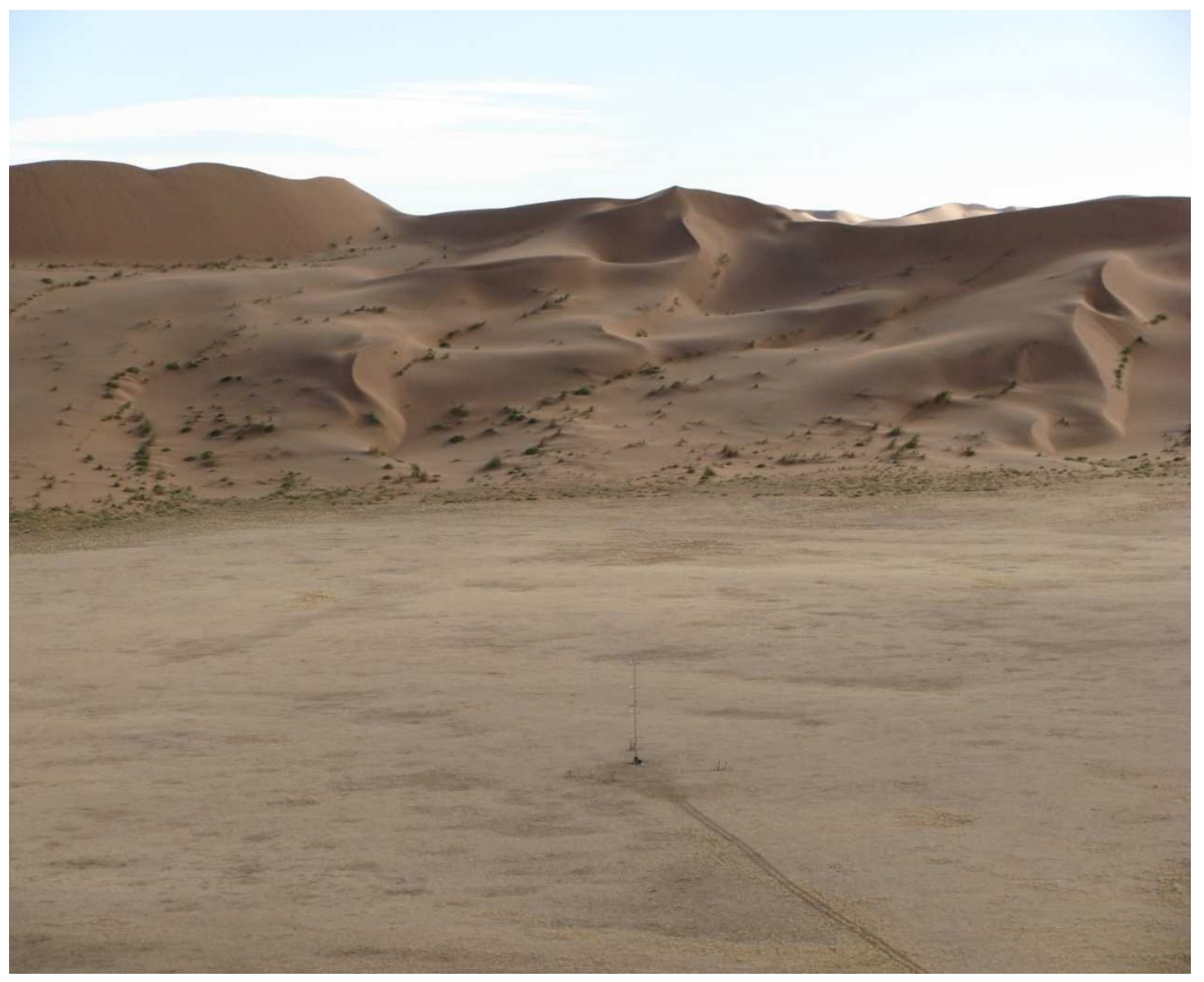

Figure 4-1: Interdune site with wind mast

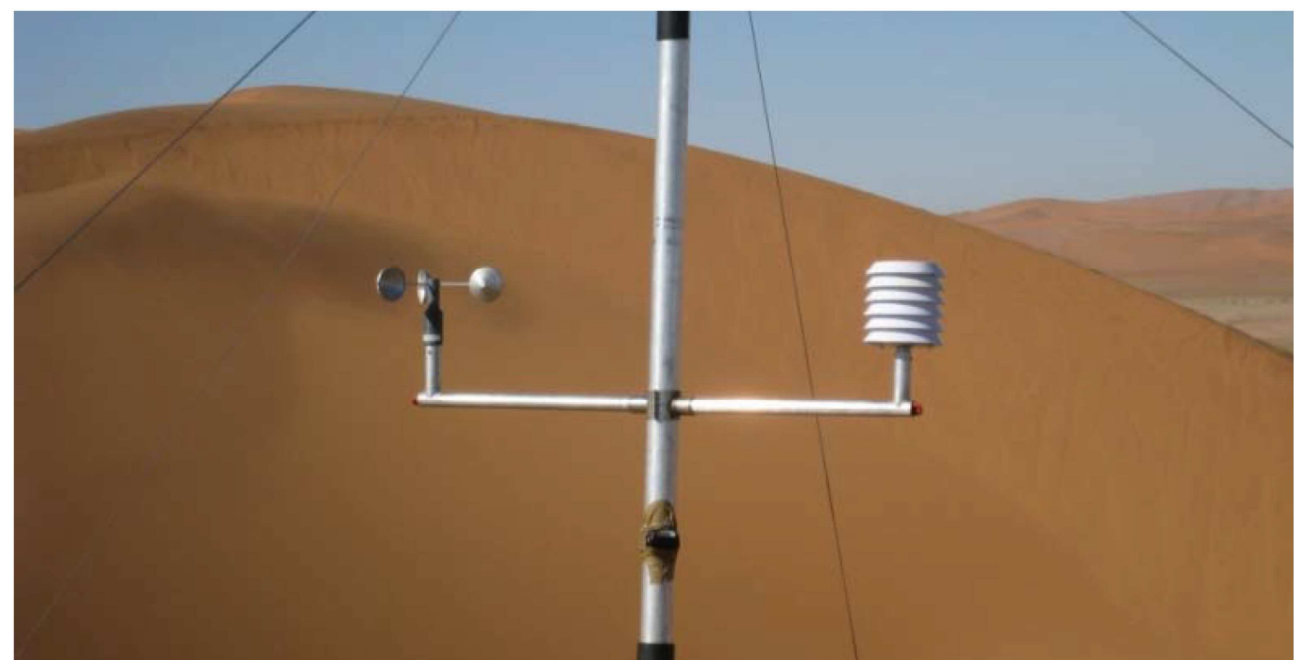

Figure 4-2: Wind speed and temperature sensor pair on wind mast 


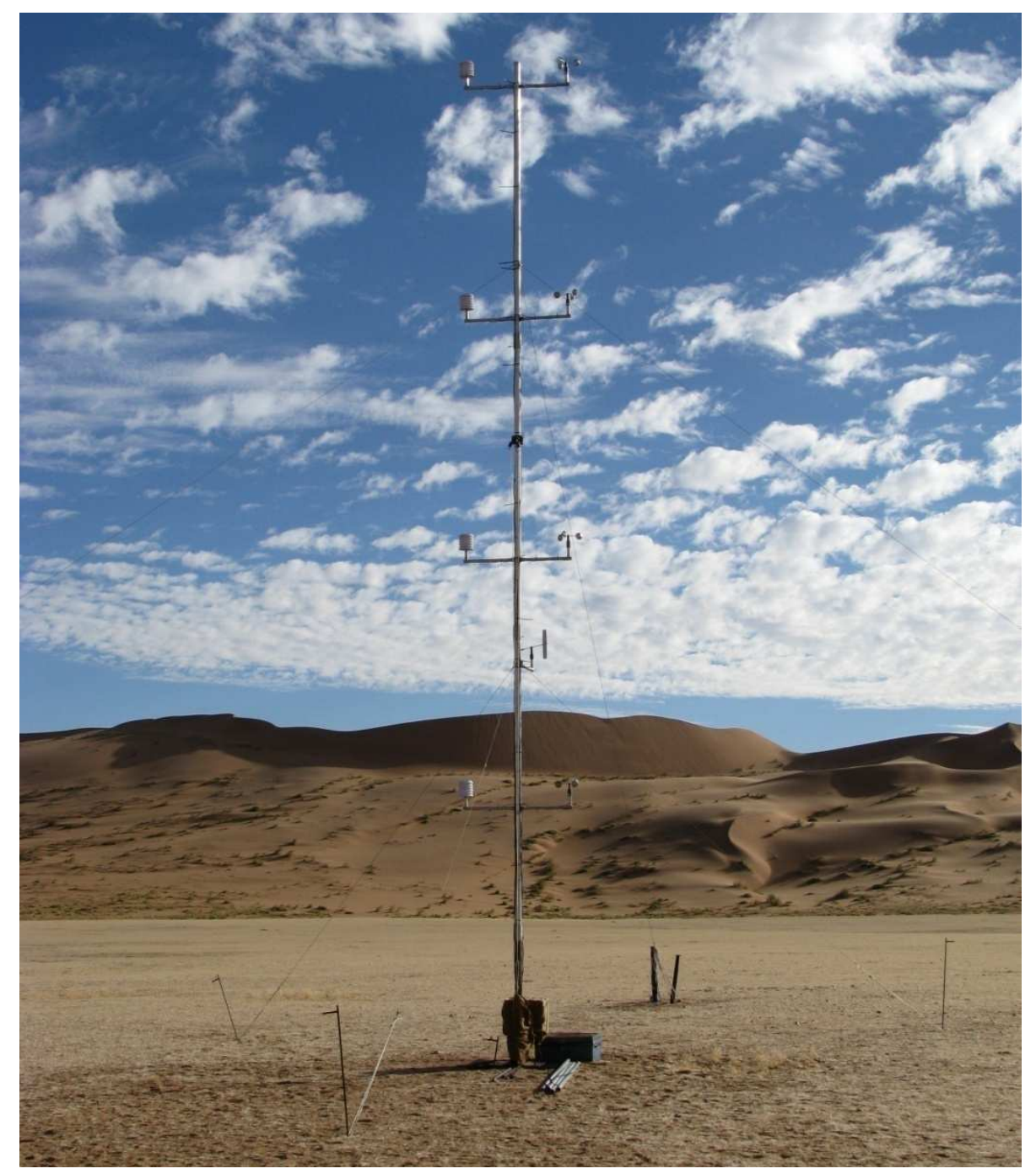

Figure 4-3: Wind mast positioned on the interdune

\subsection{Determination of Velocity and Temperature Profiles from Data}

The turbulent fluxes of momentum and heat near the surface are of primary concern in micrometeorology, as they determine the shapes of the vertical profiles of velocity and temperature. The data acquired from the wind mast could now be used to determine these parameters. The data was processed using the profile method which requires measurements of wind speed and temperature at more than two heights in the surface layer. The appropriate flux-profile relations can be fitted to the observations using the least square technique (Arya 2001b).

The analysis was limited to data points obtained when the wind was blowing from a predominantly Southerly or Northerly direction. The reason for this is that the dunes were orientated with their longitudinal length in a North-South direction and therefore, for the mast placed in the interdune, the greatest upstream fetch 
available for the wind to adapt to the surface conditions was when it was blowing from the North or the South. These profiles were filtered from the logged data using Microsoft Excel (2010) and saved as CSV (comma delimited) files. Profiles within a $30^{\circ}$ arc North and South were used. Further data processing was performed using the numerical computing environment MATLAB (The MathWorks Inc. 2010).

The following describes the method for determining fluxes from measurements of gradients of temperature and velocity between any two heights $z_{1}$ and $z_{2}$ (where $z_{2}>z_{1}$ ). First, the logarithmic finite-difference approximation method for vertical gradients of any mean micrometeorological variable $M$ is:

$$
\left(\frac{\partial M}{\partial \ln z}\right)_{z_{m}} \cong \frac{\Delta M}{\Delta \ln (z)}=\frac{M_{2}-M_{1}}{\ln \left(z_{2} / z_{1}\right)}
$$

or

$$
\left(\frac{\partial M}{\partial \mathrm{z}}\right)_{z_{m}} \cong \frac{\Delta M}{z_{m} \ln \left(z_{2} / z_{1}\right)}
$$

which is applicable at:

$$
z_{m}=\left(z_{1} z_{2}\right)^{1 / 2}
$$

the geometric mean height. Now using the logarithmic finite-difference approximation for the velocity and potential temperature gradients, the Richardson number at $z_{m}$ can be given as:

$$
R i_{m}=\frac{g}{T_{1}} \frac{(\partial \theta / \partial z)_{z_{m}}}{(\partial u / \partial z)_{z_{m}}^{2}}=\frac{g}{T_{1}} \frac{\Delta \theta z_{m}}{(\Delta u)^{2}} \ln \left(\frac{z_{2}}{z_{1}}\right)
$$

This requires the transformation of the measured temperature to potential temperature using Equation 2-18. The Richardson number can now be calculated from the measurements of $u$ and $\theta$ at each pair of consecutive levels and an estimate of the Monin-Obukhov length $L$ can be obtained from a straight line fit through the data points of $z_{m}$ versus $R i$ for unstable conditions, or $z_{m}$ versus $R i /(1-5 R i)$ for stable conditions, using least squares. In both cases, according to Equations 2-37 and 2-38, $L$ will be equal to the slope of the best fitted line.

The next step is to plot $u$ versus $\ln (z)-\psi_{m}(z / L)$ and $\theta$ versus $\ln (z)-\psi_{h}(z / L)$ and to again use least squares to fit a straight line through the data points. According to Equations 2-39 and 2-40, the slopes of these lines must be $\kappa / u_{*}$ and $\kappa / \theta_{*}$, from which $u_{*}$ and $\theta_{*}$ can be determined. The surface fluxes, given by Equations 2-31 and 2-32, repeated here for convenience: 


$$
\begin{gathered}
\tau_{0}=\rho u_{*}^{2} \\
\dot{q}_{0}=-\rho c_{p} u_{*} \theta_{*}
\end{gathered}
$$

can then be calculated. By rewriting Equations 2-39 and 2-40 in the following forms:

$$
\begin{gathered}
\ln (z)-\psi_{m}(z / L)=\frac{\kappa}{u_{*}} u+\ln \left(z_{0}\right) \\
\ln (z)-\psi_{h}(z / L)=\frac{\kappa}{\theta_{*}} \theta-\frac{\kappa}{\theta_{*}} \theta_{0}+\ln \left(z_{0}\right)
\end{gathered}
$$

it can be seen that the intercepts of the plotted lines of $u$ versus $\ln (z)-\psi_{m}(z / L)$ and $\theta$ versus $\ln (z)-\psi_{h}(z / L)$ can be used to determine $z_{0}$ and $T_{0}$. Furthermore, for the power law formulation of the wind velocity profile given by:

$$
\frac{u}{u_{r}}=\left(\frac{z}{z_{r}}\right)^{m}
$$

where $u_{r}$ is the reference velocity, the exponent $m$, as expressed as a function of surface roughness and stability, can be given by:

$$
m=\varphi_{m}\left(\zeta_{r}\right) /\left[\ln \left(\frac{z_{r}}{z_{0}}\right)-\psi_{m}\left(\zeta_{r}\right)\right]
$$

where:

$$
\zeta_{r}=z_{r} / L
$$

and $z_{r}$ is the reference height, usually taken as $10 \mathrm{~m}$. The value of this exponent is about 0.2 for a moderately rough surface under neutral conditions (Arya 2001a). As already mentioned, the measured data was analysed by the method stated above using MATLAB, the full code for which is given in Appendix A. A sample calculation of this procedure is given in Appendix B.

It should further be noted that when going through the procedure of determining $z_{0}$ and $u_{*}$ it is advisable that the observed levels be situated at sufficiently small values of $z / L$ to ensure that they lie within the logarithmic portion of the profile (Blackadar 1997). For this reason only profiles that yielded values of $L \geq|5|$ were considered, so that at least half of the mast was in the portion of the boundary layer dominated by shear turbulence production. Additionally, if there are several 
wind profiles available, one can determine $z_{0}$ from the geometric mean of the values obtained from individual profiles (Arya 2001a).

\subsection{Results and Discussion}

The results of the analysis of the measured profiles are given in Table 4-1 for stable conditions and Table 4-2 for unstable conditions. No stable conditions were recorded for winds blowing from the Northerly direction and no neutral atmospheric conditions were present during the time that the measurements were made. It is noted that only profiles that gave $|L|>5 \mathrm{~m}$ are presented, and therefore highly stable and unstable profiles were excluded, as the accuracy of the method for such profiles is not guaranteed.

The first thing to notice from examination of Table 4-1 is that stable conditions occurred almost exclusively during the night time, between 19:40 in the evening and 08:10 in the morning. The temperature (given in ${ }^{\circ} \mathrm{C}$ ) and velocity (given in $\mathrm{m} / \mathrm{s}$ ) profiles have $T_{1}$ and $u_{1}$ respectively corresponding to the measurement height $z_{1}$, with $z_{2}>z_{1}$. It can be seen that the temperature readings for the stable conditions consistently increase with increasing measurement height, indicating a surface temperature inversion. The maximum value of $|L|$ for stable conditions was found to be $58.81 \mathrm{~m}$ and the maximum heat flux $\left(\dot{q}_{0}\right)$ into the ground was found to $117.80 \mathrm{~W} / \mathrm{m}^{2}$, with the average being $26.53 \mathrm{~W} / \mathrm{m}^{2}$. The average power law exponent $m$ for stable conditions was found to be 0.5 .

Examination of Table 4-2 for the unstable conditions reveals that these profiles were measured exclusively during the day, between 09:00 in the morning and 15:00 in the afternoon. The temperatures were found to consistently decrease with increasing measurement height. The maximum value of $|L|$ for unstable conditions was found to be $60.56 \mathrm{~m}$ and the maximum heat flux from the ground to the air was calculated as $579.98 \mathrm{~W} / \mathrm{m}^{2}$. The average heat flux under unstable conditions was $207.34 \mathrm{~W} / \mathrm{m}^{2}$. It was additionally found that the average power law exponent $m$ under unstable conditions was 0.1 . Moreover the average recorded velocity at the $10 \mathrm{~m}$ measuring height was in the order of $5 \mathrm{~m} / \mathrm{s}$ for stable and unstable profiles. The geometric mean value of $z_{0}$ for all the profiles was found to have a value of $0.015 \mathrm{~m}$, with a minimum value of $0.0003 \mathrm{~m}$ and a maximum of $0.4 \mathrm{~m}$.

Plots of selected velocity and temperature profiles for a stable and unstable recording are shown in Figure 4-4 and Figure 4-5 respectively, with the values given in Table 4-3. Good correspondence is seen between the measurements and the predicated profiles. It can be seen that the stable velocity becomes increasingly linear with increasing height while the velocity under unstable conditions becomes more curvilinear. The plots of temperature are shown next to the predicted adiabatic lapse rate for the same surface temperature and reveal clearly the temperature inversion under the stable conditions and the near surface superadiabatic layer under unstable conditions. 


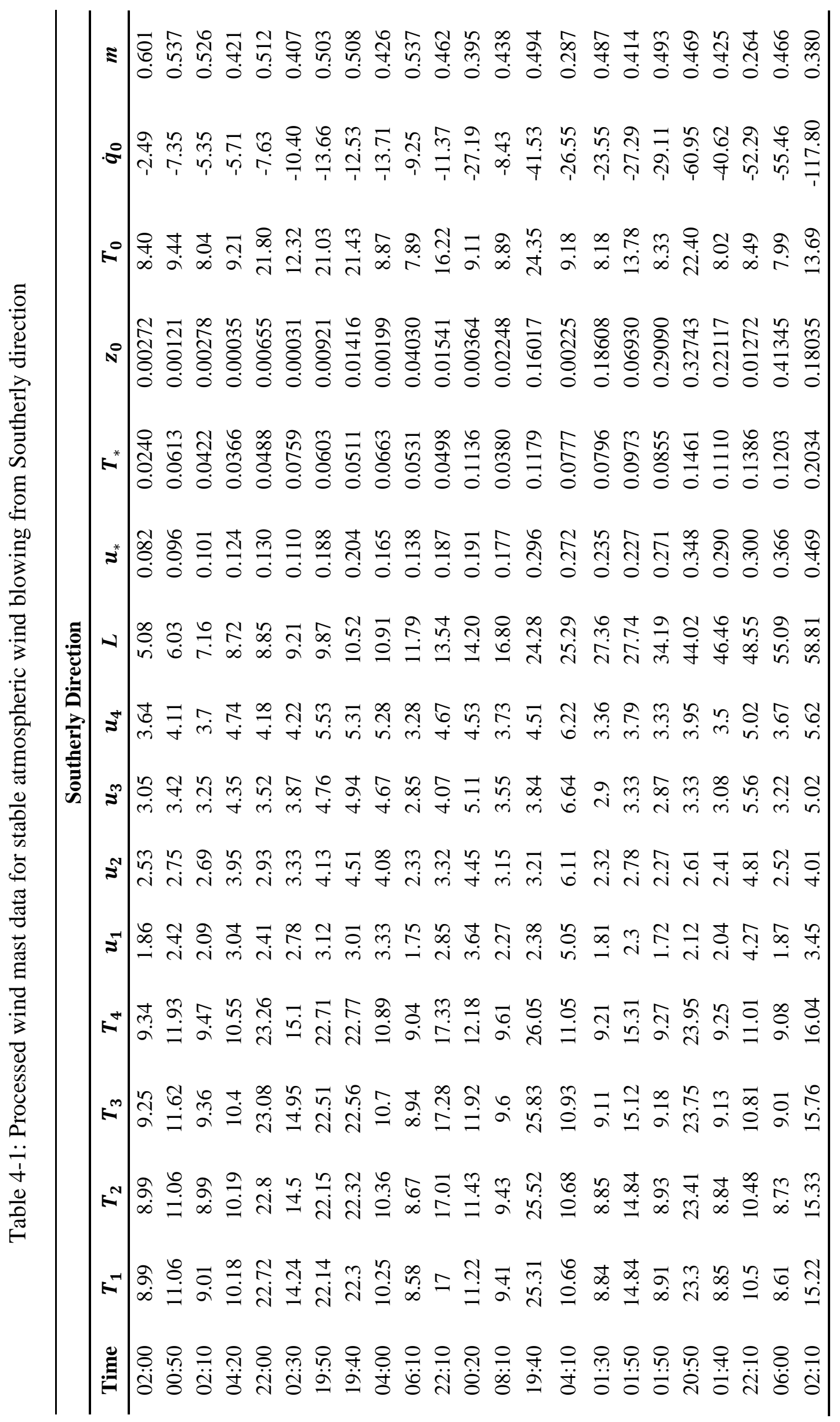




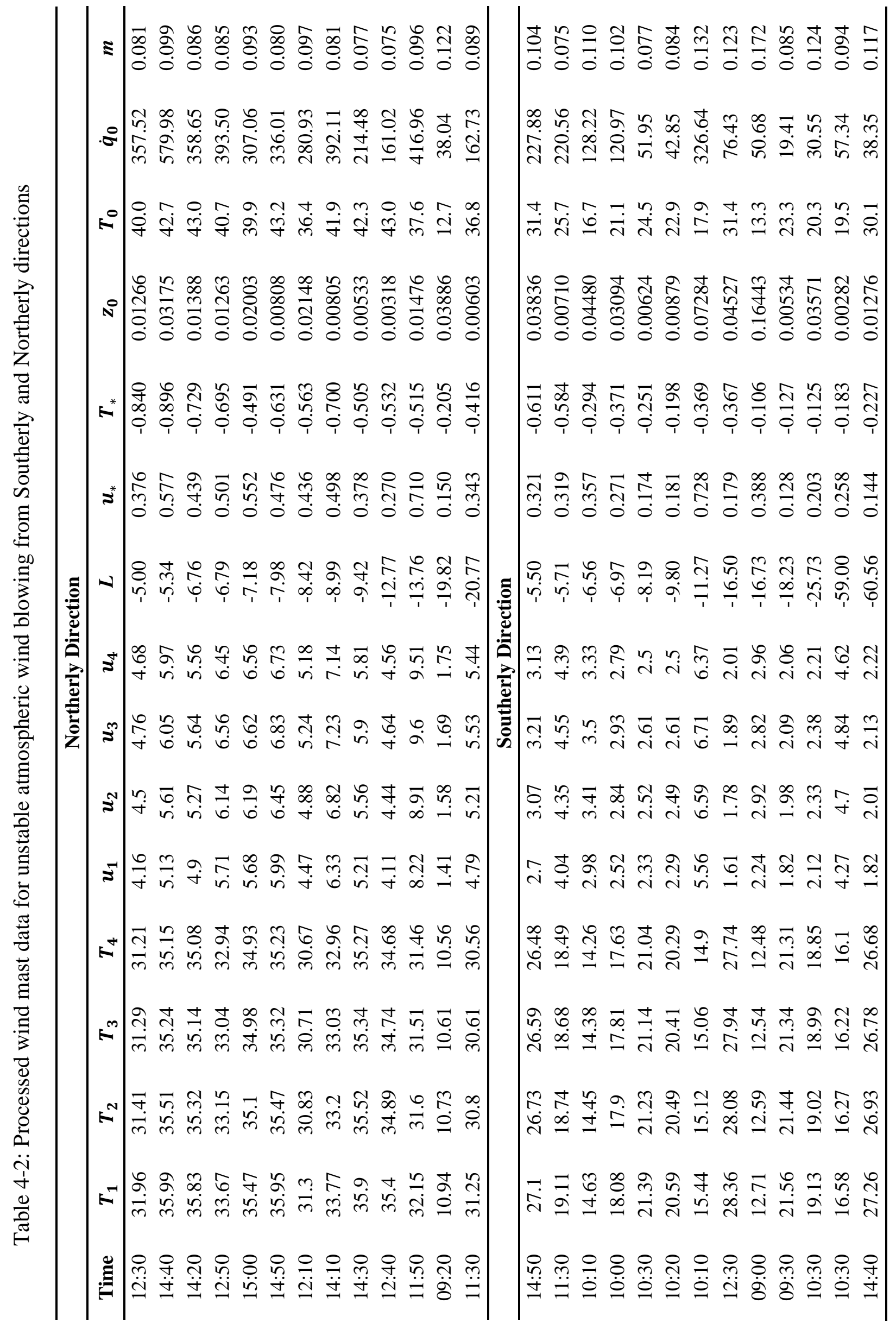


Table 4-3: Selected profiles for plotting

\begin{tabular}{ccccccccccc}
\hline $\boldsymbol{T}_{\mathbf{1}}$ & $\boldsymbol{T}_{\mathbf{2}}$ & $\boldsymbol{T}_{\mathbf{3}}$ & $\boldsymbol{T}_{\mathbf{4}}$ & $\boldsymbol{u}_{\mathbf{1}}$ & $\boldsymbol{u}_{\mathbf{2}}$ & $\boldsymbol{u}_{\mathbf{3}}$ & $\boldsymbol{u}_{\mathbf{4}}$ & $\boldsymbol{L}$ & $\boldsymbol{z}_{\mathbf{0}}$ & $\boldsymbol{T}_{\boldsymbol{0}}$ \\
\hline 10.25 & 10.36 & 10.7 & 10.89 & 3.33 & 4.08 & 4.67 & 5.28 & 10.9 & 0.00199 & 8.87 \\
35.4 & 34.89 & 34.74 & 34.68 & 4.11 & 4.44 & 4.64 & 4.56 & -12.8 & 0.00318 & 43.0 \\
\hline
\end{tabular}

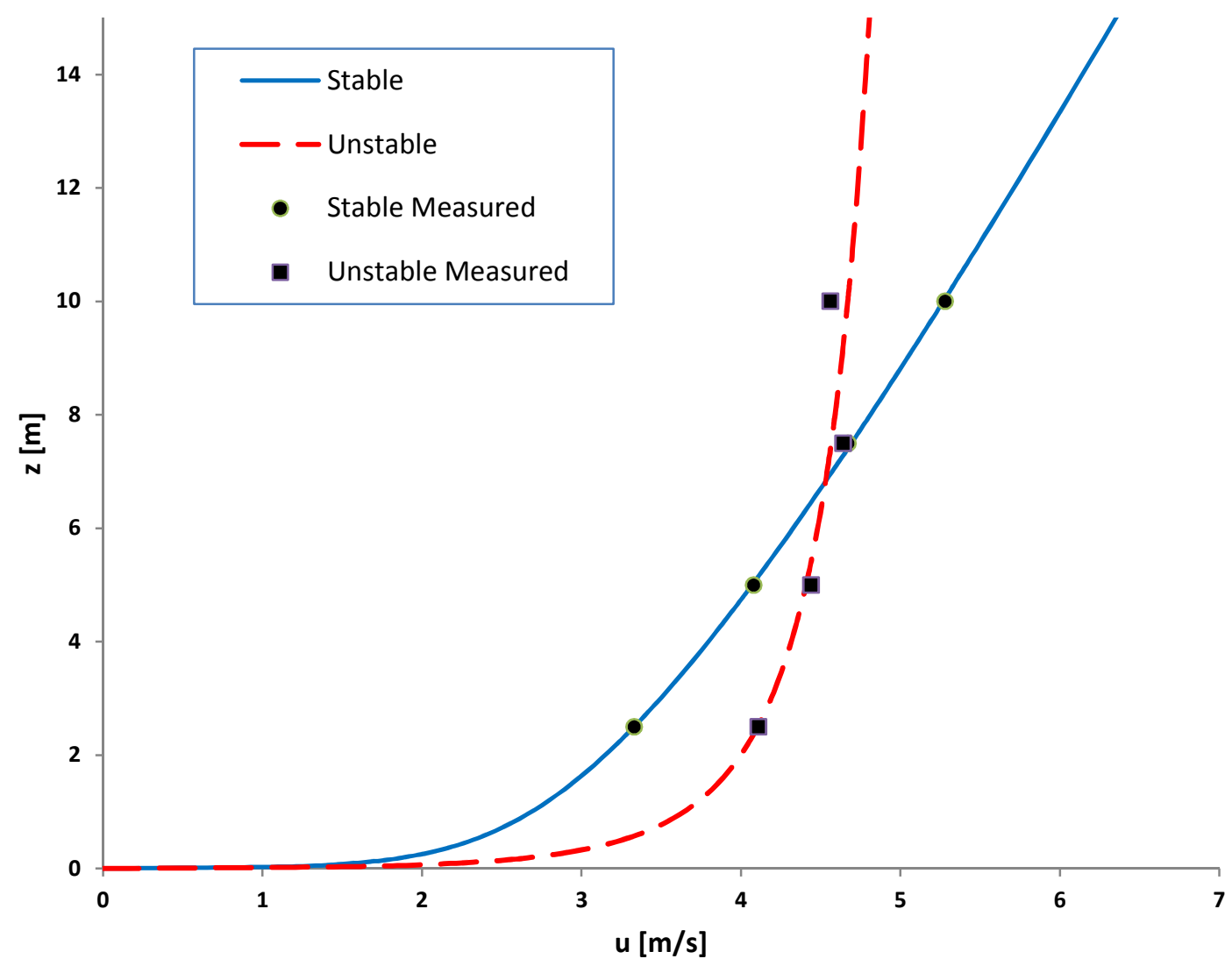

Figure 4-4: Measured data and predicted profiles for velocity

What the results reveal is that the measured profiles adhere well to the predictions of Monin-Obukhov theory and are consistent with the idea of diurnal cycling between stable conditions, predominating at night, and unstable conditions occurring during the day, with neutral conditions being rare. We, however, observed a gap in the time data, with no stable or unstable profiles measured between the times of 15:00 in the afternoon and 19:30 in the evening.

It should be noted that in the analysis of the measurements, any profile that gave inconsistent Richardson number results between the different measurement layers, i.e. at each respective value of $z_{m}$, were discarded. Thus only profiles that revealed consistently positive or negative Richardson numbers at each value of $z_{m}$ 
were considered. Any profile then that was in transition between stable and unstable conditions, such as under conditions of surface cooling while the air above was still in the unstable regime, would not have been recorded in the results presented here. Typically such a transition would occur during the late afternoon as the sun starts setting and conditions cool down, thus explaining the gap in the time data.

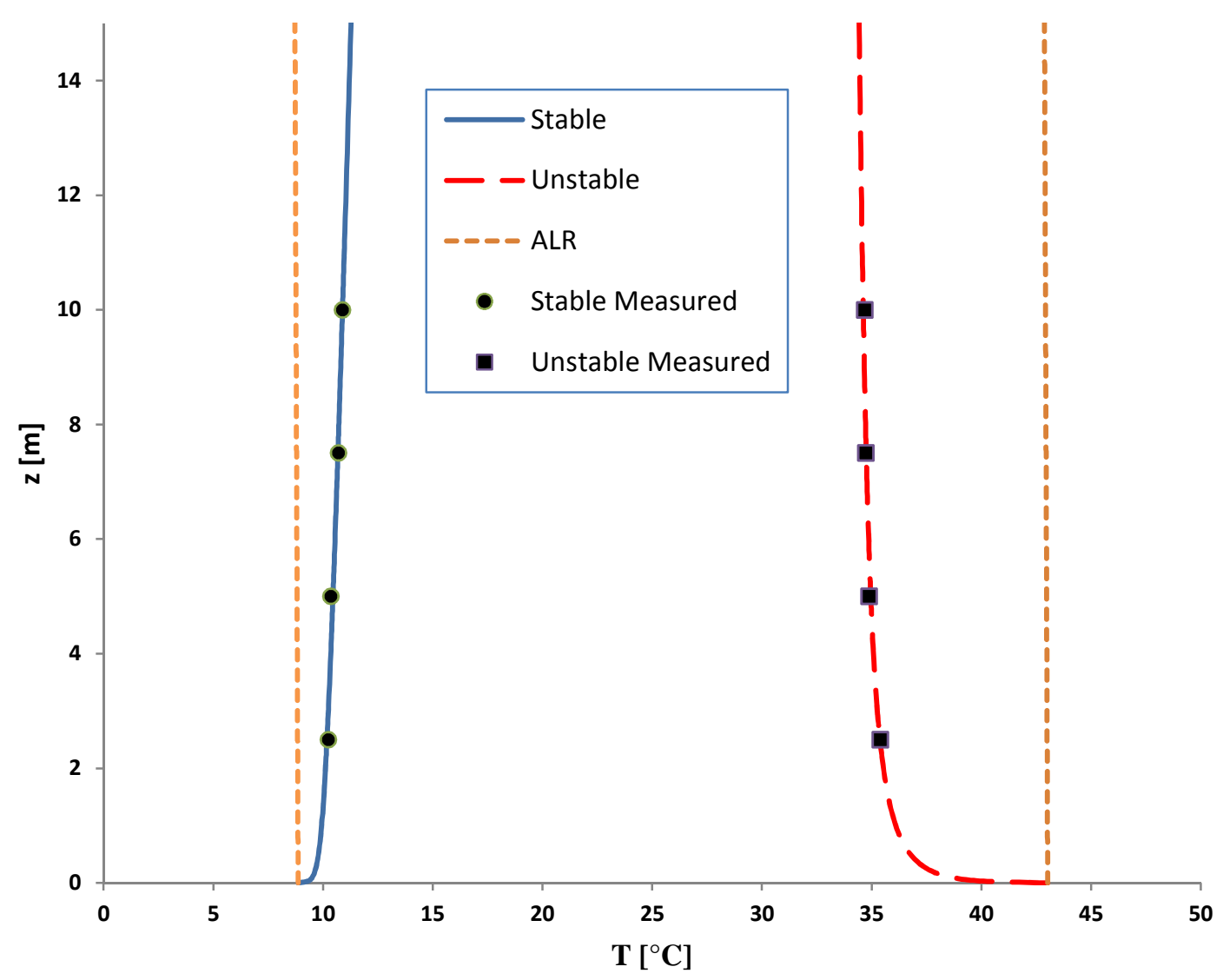

Figure 4-5: Measured data and predicted profiles for temperature

The maximum value of $|L|$ was about $60 \mathrm{~m}$. What this shows is that the shear dominated region is quite small, and therefore the assumption of neutral logarithmic conditions can at best be extrapolated to a height of about $60 \mathrm{~m}$, showing that for flow analysis in such desert like conditions one must account for the thermally stratified atmosphere. Buoyant production of turbulence therefore dominates the $\mathrm{ABL}$ in such environments to a large extent.

It is interesting to note that if the surface heat flux under unstable conditions is large, the temperature gradients close to the surface are also large. The gradient can be calculated by solving Equation 2-30 for $\partial \theta / \partial z$. For a strongly heated surface with $\dot{q}_{0}$ in the order of $600 \mathrm{~W} / \mathrm{m}^{2}, u_{*}$ of $0.4, L$ of $-10 \mathrm{~m}$ and $T_{*}$ of $-1.25^{\circ} \mathrm{C}$, the gradient at a height of $0.1 \mathrm{~m}$ is calculated to be $-34.0^{\circ} \mathrm{C} / \mathrm{m}$ (Blackadar 1997). 
This is about 3400 times the adiabatic lapse rate and results in a strong upward increase in density. The light entering this layer of changing density horizontally is strongly refracted upward, which creates the possibility for the formation of mirages, or a phenomenon known as the fata morgana, the shimmering of light above the horizon on a hot day (Blackadar 1997).

It was further observed that most of the roughness length values fell between the "smooth" and "open" Davenport classifications for effective terrain roughness, where "smooth" defines featureless land surface without any noticeable obstacles and negligible vegetation $\left(z_{0}\right.$ in the order of $\left.10^{-3}\right)$ and "open" defines level country with low vegetation (e.g. grass) and isolated obstacles with separations of at least 50 obstacle heights $\left(z_{0}\right.$ in the order of $10^{-2}$ ) (Wieringa 2001). This is consistent with the description of the interdune area. Furthermore, as far as the power law description of the velocity profile is concerned, values of about 0.1 are expected for unstable conditions over moderately rough surface, while stable conditions should be about 0.6 in magnitude (Arya 2001b). This too is consistent with the calculated values from the observed measurements.

It can be concluded that the profiles predicted by the Monin-Obukhov similarity theory are readily measured in the interdune area of the Namib Desert, and it is therefore imperative that the thermally stratified ABL be considered in any flow analysis involving such regions. The results proved to be consistently in line with the theory and provided the anticipated outcomes. This method also proves to be appropriate for the determination of aerodynamic roughness length, as it accounts for the potential thermal stratification of the atmosphere and does not rely on the assumption of neutral conditions, as is often the case when $z_{0}$ is derived from measured wind profiles.

The parameters thus derived from the measured profiles using Monin-Obukhov similarity theory describe these profiles well and can be used to describe nearly any potential thermal state of the atmosphere accurately and completely. This is provided that the surface layer is in equilibrium with the surface fluxes and has adapted to them. Profiles in transition, however, need to be accounted for by another method. This illustrates the importance of having a substantial fetch available upstream of the wind mast if one is to accurately calculate the surface fluxes from the measured profiles of wind velocity and temperature, and justifies the decision made in this study to only consider profiles recorded from the Southerly or Northerly directions. 


\section{CFD SIMULATION OF THE ABL UNDER DIFFERENT THERMAL STRATIFICATIONS}

We now turn our attention to applying the ABL and Monin-Obukhov theory to CFD simulations. The goal here is to model the ABL under different thermal stability conditions using the appropriate boundary conditions and to maintain the flow property profiles along an extensive fetch of the flow domain. The gravity effects must be accounted for in the transport equations and full variation in density, temperature and pressure must be achieved. Two different RANS two equation models were tested, namely the standard $k-\varepsilon$ model and the SST $k-\omega$ model, for their ability to model the flow. Both allow for the buoyant production of turbulence to be implemented. The commercial code STAR-CCM+ was employed.

The relevant parameters of the boundary layers that are to be simulated are given in Table 5-1. Stable, unstable and neutral atmospheres are to be modelled. The value specified for the aerodynamic roughness length corresponds to the "smooth" Davenport classification, applicable to featureless land surface without any noticeable obstacles and with negligible vegetation (Wieringa 2001). Furthermore the equivalent sand-grain roughness height was calculated using:

$$
k_{s}=7.5 z_{0}
$$

which is chosen to correspond closely to Equation 3-5 for the obstruction height of various types of crops and grass-land, and allows for better grid resolution close to the ground. All the profiles were chosen to have a reference velocity $\left(u_{r}\right)$ of $10 \mathrm{~m} / \mathrm{s}$ at a reference height $\left(z_{r}\right)$ of $10 \mathrm{~m}$, for easy comparison.

Table 5-1: Parameters describing the different stratified atmospheres

\begin{tabular}{lccccccccc}
\hline & $\begin{array}{c}\mathbf{z}_{\mathbf{0}} \\
{[\mathrm{m}]}\end{array}$ & $\begin{array}{c}\boldsymbol{u}_{\boldsymbol{r}} \\
{[\mathrm{m} / \mathrm{s}]}\end{array}$ & $\begin{array}{c}\boldsymbol{z}_{\boldsymbol{r}} \\
{[\mathrm{m}]}\end{array}$ & $\begin{array}{c}\dot{\boldsymbol{q}}_{\mathbf{0}} \\
{\left[\mathrm{W} / \mathrm{m}^{2}\right]}\end{array}$ & $\begin{array}{c}\boldsymbol{T}_{\mathbf{0}} \\
{\left[{ }^{\circ} \mathrm{C}\right]}\end{array}$ & $\begin{array}{c}\mathbf{L} \\
{[\mathrm{m}]}\end{array}$ & $\begin{array}{c}\boldsymbol{u}_{*} \\
{[\mathrm{~m} / \mathrm{s}]}\end{array}$ & $\begin{array}{c}\boldsymbol{\kappa} \\
/\end{array}$ & $\begin{array}{c}\boldsymbol{k}_{\boldsymbol{s}} \\
{[\mathrm{m}]}\end{array}$ \\
\hline Neutral & 0.002 & 10 & 10 & 0 & 25 & $\infty$ & 0.481 & 0.41 & 0.015 \\
Stable & 0.002 & 10 & 10 & -30 & 10 & 309.5 & 0.472 & 0.41 & 0.015 \\
Unstable & 0.002 & 10 & 10 & 100 & 40 & -108.1 & 0.497 & 0.41 & 0.015 \\
\hline
\end{tabular}

\subsection{Computational Domain and Grid Generation}

The computational domain was a two dimensional empty rectangle $500 \mathrm{~m}$ in height and $5000 \mathrm{~m}$ in length (Hargreaves and Wright 2007, Blocken et al. 2007b, Parente et al. 2011b), and is shown schematically in Figure 5-1. A threedimensional STL (stereo lithography) surface file was created with dimensions of $500 \mathrm{~m}$ high, $5000 \mathrm{~m}$ long and $50 \mathrm{~m}$ wide, using CAD software. This surface file could then be imported into STAR-CCM+ as a new fluid region, meshed and converted into a two-dimensional grid. 
The region was split into boundaries with the left vertical boundary face, as shown in Figure 5-1, being specified as a "velocity inlet". This boundary would serve as the point where the inlet conditions could be specified. The right vertical boundary face was chosen to be a "flow-split outlet", where all the flow is assumed to exit from and the horizontal gradients are zero, i.e. the exit flow is assumed to be fully developed (CD-adapco Inc. 2011). The bottom boundary was a wall and the top boundary was selected as a "velocity inlet", as this allows for the fluid properties at this height to be specified, such as the velocity, temperature and turbulence parameters, and can serve to drive the flow (Franke et al. 2011, Blocken et al. 2007a, b). The side boundaries in the three-dimensional fluid domain were specified as symmetry planes.

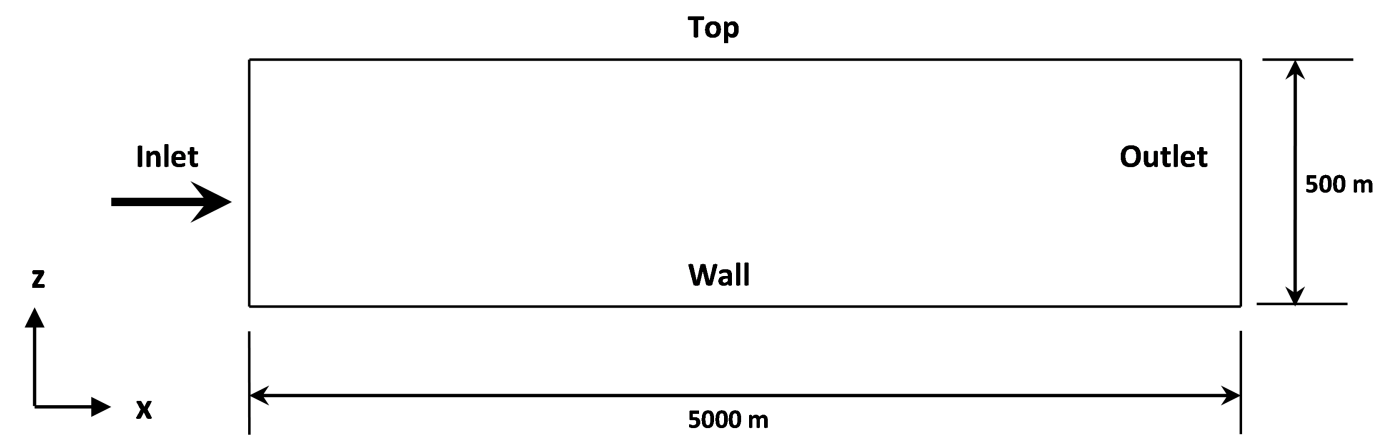

Figure 5-1: Schematic of computational domain (not to scale)

The surface was meshed using the "trimmer" meshing model which produces a predominantly hexahedral mesh with minimal cell skewness, implements automatic curvature and proximity refinement and displays surface quality independence (CD-adapco Inc. 2011). An additional advantage of this meshing model is that it provides the user the ability to automatically refine cells in a wake region. The "trimmer wake refinement" refines cells by sweeping a specified surface in a specified direction for a specified distance and refining the cells to a specified level (CD-adapco Inc. 2011).

The "surface remesher" was also implemented which re-triangulates an existing surface to improve the overall quality and optimise it for volume mesh models (CD-adapco Inc. 2011). In addition a "prism layer mesher" was used to add orthogonal prismatic cells next to wall boundaries in the volume mesh. They are required to refine a mesh close to a wall and therefore assist in the accurate simulation of turbulence and heat transfer (CD-adapco Inc. 2011). The reference values used in the meshing models are listed in Table 5-2.

It was furthermore decided to use a grid size that is similar to the grids used in practice, and therefore the mesh was further refined near the surface using the "trimmer wake refinement" option. The ground was used as the reference boundary and was swept for $100 \mathrm{~m}$ in the vertical $(z)$ direction. The inputs for this model are given in Table 5-3. 
Table 5-2: Inputs for meshing model

\begin{tabular}{llc}
\hline Reference Node Name & Property Name & Value \\
\hline Base Size & Value & $10 \mathrm{~m}$ \\
CAD Projection & Project to CAD & True (default) \\
Maximum Cell Size>Relative Size & Percentage of Base & $100 \%$ \\
Number of Prism Layers & Number of Prism Layers & 25 \\
Prism Layer Stretching & Prism Layer Stretching & 1.17 \\
Prism Layer Thickness $>$ Relative Size & Percentage of Base & $100 \%$ \\
Surface Curvature & \# Pts/circle & 36 (default) \\
Surface Growth Rate & Surface Growth Rate & 1.3 (default) \\
Surface Proximity & \# Points in a gap & 2.0 (default) \\
Surface Proximity & Search Floor & 0.0 (default) \\
Surface Size $>$ Relative Minimum Size & Percentage of Base & 25 \\
Surface Size $>$ Relative Target Size & Percentage of Base & $100 \%$ \\
Template Growth Rate & Default Growth Rate & Fast (default) \\
Template Growth Rate & Boundary Growth Rate & None (default) \\
\hline
\end{tabular}

The resulting three-dimensional mesh had 577000 cells with a $z_{p}$ of $0.0171 \mathrm{~m}$, so that $z_{p}>k_{s}$, and could now be converted into a two-dimensional mesh by removing the thickness of the domain, and therefore also the symmetry boundaries specified for the sides of the three-dimensional domain. The resulting two-dimensional mesh consisted of 65500 cells, a section of which is shown in Figure 5-2.

Table 5-3: Inputs for mesh refinement

\begin{tabular}{lc}
\hline Property Name & Value \\
\hline Relative/Absolute> Relative to base & $50 \%$ \\
Boundary Growth Rate & None (default) \\
Boundaries/Feature Curves & "Ground Wall" \\
Distance & $100 \mathrm{~m}$ \\
Direction & {$[0.0,0.0,1.0]$} \\
Default Growth Rate & Fast (default) \\
\hline
\end{tabular}

The resulting mesh was used for all the subsequent CFD calculations in this section. The grid had similar to finer resolution as grids used to investigate the ABL in other studies (Hargreaves and Wright 2007, Blocken et al. 2007b, Parente et al. 2011b), and it was also observed that the findings were consistent for any 
grid of reasonable resolution. The refinement, as stated, was chosen to correspond to the level of refinement of the grid for flow approaching an object in the fluid domain. The two-dimensional simulation, however, does not truly represent threedimensional turbulence but it does serve the purpose of illustrating the problem economically in terms of required computing power (Blocken et al. 2007b).

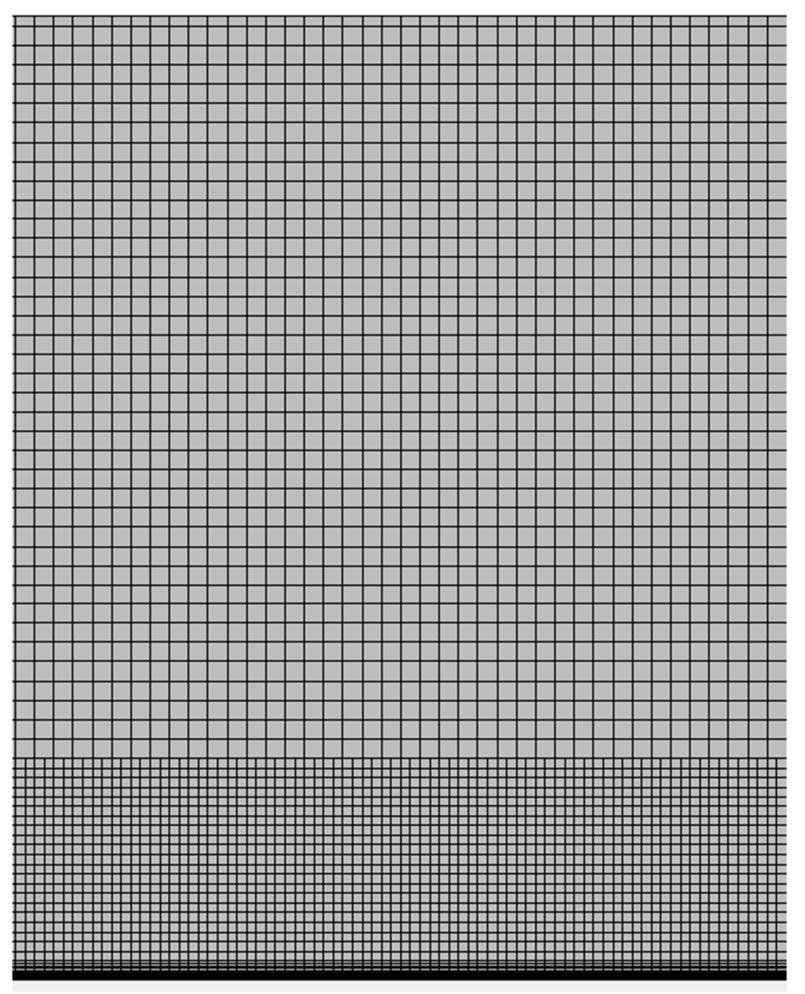

Figure 5-2: Section of computational mesh used in ABL simulation

\subsection{Flow Physics Solution Specification}

\subsubsection{Physics models and solver}

The physics models used to specify the fluid were as follows: the fluid space was specified as two-dimensional and the time specification was steady. The material specified was air with the following properties: constant dynamic viscosity $(\mu)$ of $1.85508 \times 10^{-5}$ Pa.s; constant molecular weight $(M)$ of $28.9664 \mathrm{~kg} / \mathrm{kmol}$; constant specific heat $\left(c_{p}\right)$ of $1003.62 \mathrm{~J} / \mathrm{kg} . \mathrm{K}$; constant thermal conductivity $(\lambda)$ of $0.0260305 \mathrm{~W} / \mathrm{m} . \mathrm{K}$; and a turbulent Prandtl $\left(\sigma_{T}\right)$ number of 1.0 . The density was calculated through the ideal gas law. The segregated flow solver was chosen, which achieves pressure-velocity coupling through the SIMPLE algorithm, and solves the total energy equation through the segregated fluid temperature model, which uses temperature as the independent variable (CD-adapco Inc. 2011). Convection of momentum and energy was solved using the second-order upwind 
scheme. The turbulent viscous regime was chosen with turbulence modelled using the Reynolds-Averaged Navier-Stokes (RANS) turbulence models.

Two different RANS models were investigated, namely the standard $k$ - $\varepsilon$ model and the SST $k-\omega$ model. In both cases the high $z^{+}$wall treatment was used which represents the classic wall function approach (note that $z^{+}$is referred to as $y^{+}$in fluid mechanics literature, but is renamed for consistency with the coordinate system used in this study), where the wall shear stress and turbulence parameters are all derived from equilibrium turbulent boundary layer theory, and requires that the near wall cell lies within the logarithmic region of the boundary layer (CDadapco Inc. 2011). It thus requires that $z^{+}$be between the values of 30 and 500 . The $z_{p}(0.0171 \mathrm{~m})$ for the computational mesh used in this analysis yielded a $z^{+}$ value of about 450 along the length of the bottom wall, thereby satisfying both the requirement of $z_{p}>k_{s}$ and $z^{+}<500$.

A reference pressure of $101325 \mathrm{~Pa}$ was used, which is applied by default at the point $[0,0]$ in the fluid domain (CD-adapco Inc. 2011). The flow was initialised using the inlet boundary conditions for velocity, temperature, and turbulence quantities defined below, as well as zero pressure. The solver employed the Gauss-Seidel relaxation scheme with under-relaxation factors given in Table 5-4. The solution was run until the normalised residuals of continuity, momentum, energy and turbulence had converged.

Table 5-4:Under-relaxation factors for flow simulations

\begin{tabular}{ll}
\hline Pressure & 0.3 \\
Velocity & 0.7 \\
Energy & 0.9 \\
Turbulence & 0.8 \\
Turbulent Viscosity & 1.0 \\
\hline
\end{tabular}

\subsubsection{Specification of source terms}

This still left the effects of gravity to be implemented into the flow physics, as it is not assumed to be present by default in the governing equations solved by the CFD code. The terms accounting for the gravity and buoyancy effects could be added through the specification of custom source terms, specified using the code's "user field function" application. What is more, the requirement that temperature be conserved with height so that the adiabatic nature of the real atmosphere can be modelled still has to be addressed. This, as discussed in Chapter 3, can be implemented by converting the temperature that the code uses into potential temperature, by adding a corrective heat source to the energy equation. The continuity and momentum equations adjusted for the real atmosphere are thus: 


$$
\begin{gathered}
\int_{A} \rho u_{i} n_{i} d A=0 \\
\int_{A} \rho u_{j} u_{i} n_{i} d A=-\int_{A} P \delta_{i j} n_{i} d A+\int_{A} \tau_{i j} n_{i} d A+\int_{v} S_{m} d V
\end{gathered}
$$

where $S_{m}$ is the momentum vector source term that adds the force of gravity to the volume of the fluid and therefore can be seen to add weight to the fluid. It is defined as:

$$
S_{m}=\rho g_{i}
$$

Here $g_{i}$ is the vector of gravitational acceleration, which can also be defined as a user "vector field function". The source term added to the momentum equation therefore accounts for the weight of the fluid and from inspection of Equation 5-3 it can be expected that the convection of momentum in the horizontal flow direction will be predominantly balanced by the stress tensor term, while the weight will mainly be balanced by the pressure term. This should allow the hydrostatic pressure to emerge. Additionally, by density variation through the ideal gas law, the buoyancy force will also emerge by the addition of this term, as any fluid volume of varying density relative to its surroundings will experience a resultant force. This method for accounting for buoyancy in the CFD code is illustrated in Appendix C.

The energy equation can be modified using the relation between true temperature gradient and potential temperature gradient (Equation 2-15). Thus the conduction (or diffusion) term of the energy equation can be changed and the full energy equation for steady flow is given as:

$$
\int_{A} \rho c_{p} T u_{i} n_{i} d A=\int_{V} S_{m} u_{i} d V+\int_{A}\left[u_{j} \tau_{i j}+\frac{c_{p} \mu_{t}}{\sigma_{T}}\left(\frac{\partial T}{\partial x_{i}}-\frac{g_{i}}{c_{p}}\right)\right] n_{i} d A
$$

The term representing the work done by the body force is automatically accounted for in the energy equation, as the code will by default add this term to the energy equation for any user defined source term specified in the momentum equation (CD-adapco Inc. 2011). The energy equation source term is specified as a scalar and is added as a volume integral. From the divergence theorem:

$$
\int_{V} \nabla \cdot S_{i} d V=\int_{A} S_{i} n_{i} d A
$$

where $S_{i}$ is a vector. It can be seen that the corrective energy source must be: 


$$
\int_{A} \frac{c_{p} \mu_{t}}{\sigma_{T}}\left(-\frac{g_{i}}{c_{p}}\right) n_{i} d A=\int_{V}\left(-\frac{c_{p} \mu_{t}}{\sigma_{T}}\right) \nabla \cdot\left(\frac{g_{i}}{c_{p}}\right) d V=\int_{V} S_{u} d V
$$

And therefore the energy source term that must be added is:

$$
S_{u}=\nabla \cdot\left(-\frac{\mu_{t}}{\sigma_{T}} g_{i}\right)
$$

Inspection of Equation 5-5 shows that the term $g_{i} / c_{p}$, the dry adiabatic lapse, will function by inducing heat transfer when the vertical temperature gradient $\partial T / \partial z$ is not equal to the dry adiabatic lapse rate. The heat transfer will therefore have a positive value for the unstable atmosphere when the vertical temperature gradient $\partial T / \partial z$ is greater than the adiabatic lapse rate and a negative value when the vertical temperature gradient $\partial T / \partial z$ is less than the adiabatic lapse rate, and the atmosphere is under stable stratification. Under neutral conditions there will be zero heat transfer and therefore adiabatic conditions. This is therefore consistent with the assumptions in the derivation of the Monin-Obukhov similarity theory, where the heat flux through the surface layer is equal to the ground heat flux and the potential temperature gradient, and therefore the temperature profile, is dependent on this heat flux. The heat flux vertically is therefore determined by the deviation of the temperature gradient from the adiabatic lapse rate.

The turbulent production (or removal) by buoyancy must still be accounted for, as the effect of gravity must be explicitly added to the turbulent transport equations in the CFD code. The formulation of the turbulent kinetic energy $(k)$ and turbulent kinetic energy dissipation rate $(\varepsilon)$ equations was given in Chapter 3. Equations 3-6 and 3-7 can be reformulated, where the effects of molecular dynamic viscosity and the production of turbulence due to compressibility at low Mach numbers has been ignored for simplicity:

$$
\begin{gathered}
\int_{A} \rho k u_{i} n_{i} d A=\int_{A} \frac{\mu_{t}}{\sigma_{k}} \frac{\partial k}{\partial x_{i}} n_{i} d A+\int_{V}\left[G_{k}+S_{k}-\rho \varepsilon\right] d V \\
\left.\int_{A} \rho \varepsilon u_{i} n_{i} d A=\int_{A} \frac{\mu_{t}}{\sigma_{\varepsilon}} \frac{\partial \varepsilon}{\partial x_{i}} n_{i} d A+\int_{V} C_{\varepsilon 1} \frac{\varepsilon}{k}\left(G_{k}\right)-C_{\varepsilon 2} \rho \frac{\varepsilon^{2}}{k}+S_{\varepsilon}\right] d V
\end{gathered}
$$

Here $S_{k}$ is the source term to be added to the $k$-equation and is related to $G_{b}$, but has a corrective turbulence source added to it. It has the form:

$$
S_{k}=\beta g_{i} \frac{\mu_{t}}{\sigma_{T}}\left(\frac{\partial T}{\partial x_{i}}-\frac{g_{i}}{c_{p}}\right)
$$


where the vertical temperature gradient $\partial T / \partial z$ has again been converted to the potential temperature gradient. The coefficient of thermal expansion $\beta$ is specified, using a scalar "field function", as the inverse of the temperature. It follows from Equation 3-7 that the source term for the $\varepsilon$-equation should also contain the buoyant production term. Using the formulation of Alinot and Masson (2005) and from Equation 3-7, we have:

$$
S_{\varepsilon}=C_{\varepsilon 1} \frac{\varepsilon}{k}\left(1-C_{\varepsilon 3}\right) S_{k}
$$

Notice again that the term $g_{i} / c_{p}$ will function by inducing turbulence when the vertical temperature gradient $\partial T / \partial z$ is greater than the dry adiabatic lapse rate and the atmosphere is in unstable stratification, while it will remove turbulence when the atmosphere is stable and the vertical temperature gradient $\partial T / \partial z$ is less than the adiabatic lapse rate (Alinot and Masson 2005). Under neutral conditions the turbulence source terms will be zero. The same form of the source term for the buoyant production of turbulent kinetic energy (Equation 5-11) can be added for the SST $k-\omega$ model, while it has been suggested (Peng and Davidson 1999) that the buoyancy term in the $\omega$-equation has the from:

$$
S_{\omega}=\left(C_{\varepsilon 3}-1\right)\left(\frac{\omega}{k}\right) S_{k}
$$

It has, however, been found that the solutions of buoyant flow are insensitive to the value of this term in the $\omega$-equation, and there is evidence that the buoyancy effect should only be reflected in the $k$-equation (Peng and Davidson 1999). For the purpose of this study then the SST $k$ - $\omega$ turbulence model will have the effect of buoyancy included only as a source term $S_{k}$ in the $k$-equation. The turbulence model constants used for the simulations are given in Table 5-5, with the $k-\varepsilon$ constants modified according to Equations 3-17 and 3-18, and the SST $k-\omega$ turbulence model constants being the default values employed in STAR-CCM+.

Table 5-5: Turbulence model constants

\begin{tabular}{lcccccccc}
\hline \multirow{2}{*}{ Standard $\boldsymbol{k}-\boldsymbol{\varepsilon}:$} & $C_{\varepsilon 1}$ & $C_{\varepsilon 2}$ & $C_{\mu}$ & $\sigma_{k}$ & $\sigma_{\varepsilon}$ & & & \\
& 1.1523 & 1.92 & 0.0333 & 1.0 & 1.3 & & & \\
\hline \multirow{2}{*}{ SST $\boldsymbol{k}-\boldsymbol{\omega}:$} & $\alpha_{1}$ & $\beta_{1}$ & $\beta_{*}$ & $\sigma_{k 1}$ & $\sigma_{\omega 1}$ & $\beta_{2}$ & $\sigma_{k 2}$ & $\sigma_{\omega 2}$ \\
& 0.31 & 0.075 & 0.09 & 0.85 & 0.5 & 0.0828 & 1.0 & 0.856 \\
\hline
\end{tabular}

The appropriate values of $C_{\varepsilon 3}$ for the $k-\varepsilon$ turbulence model must still be determined. A necessary condition on $C_{\varepsilon 3}$ is that the vertical distributions of $k$ and $\varepsilon$ given by Equations 3-14 and 3-16 are exact solutions to the $k$ - $\varepsilon$ turbulence model (Alinot and Masson 2002). This leads to the curve fit expression for $C_{\varepsilon 3}$ as a function of the stability parameter, with the coefficients of this series given in Table 5-6: 


$$
C_{\varepsilon 3}\left(\frac{Z}{L}\right)=\sum_{n=0}^{5} a_{n}\left(\frac{Z}{L}\right)^{n}
$$

Table 5-6: Coefficients for $C_{\varepsilon 3}$ (Alinot and Masson 2002)

\begin{tabular}{rrrrr}
\hline & $\mathbf{L}>\mathbf{0}$ & \multicolumn{3}{c}{$\mathbf{L}<\mathbf{0}$} \\
\hline $\boldsymbol{a}_{\mathbf{0}}$ & $\mathrm{z} / \mathrm{L}<0.33$ & $\mathrm{z} / \mathrm{L}>0.33$ & $\mathrm{z} / \mathrm{L}<-0.25$ & $\mathrm{z} / \mathrm{L}>-0.25$ \\
$\boldsymbol{a}_{\mathbf{1}}$ & 4.181 & 5.225 & -0.0609 & 1.765 \\
$\boldsymbol{a}_{\mathbf{2}}$ & 33.994 & -5.269 & -33.672 & 17.1346 \\
$\boldsymbol{a}_{\mathbf{3}}$ & -442.398 & 5.115 & -546.88 & 19.165 \\
$\boldsymbol{a}_{\mathbf{4}}$ & -6043.644 & -2.406 & -3234.06 & 11.912 \\
$\boldsymbol{a}_{\mathbf{5}}$ & 5970.776 & 0.435 & -9490.792 & 3.821 \\
\hline
\end{tabular}

On uniform terrain these expressions are the same for any value of $L$, but might not be appropriate over complex terrain (Alinot and Masson 2002). The resulting average values for $C_{\varepsilon 3}$ is 3.4 under stable conditions and -4.4 under unstable conditions (Alinot and Masson 2005). The expression for $C_{\varepsilon 3}$ given by Equation 5-14 was found to work well for stable conditions, but induced numerical instability for unstable conditions. For unstable conditions a constant value of -4.4 was used.

\subsubsection{Boundary conditions}

The "velocity inlet" or inlet boundary was the $z$ line located upstream of the fluid domain. The static temperature, velocity components $\left(u_{x}\right.$ equal to $u(z)$ with zero $u_{y}$ and $u_{z}$ ) and turbulence properties were specified here using the "Table (x, y, z)" method, where the properties along the vertical height of the domain was calculated in Microsoft Excel and exported in comma delimited (CSV) format, from where they could be imported into STAR-CCM+. The velocity and temperature profiles given by Equations 2-39 and 2-40, are multiplied out in full and used to specify the inlet velocity and temperature (Alinot and Masson 2002):

$$
u(z)=\left\{\begin{array}{cc}
\frac{u_{*}}{\kappa}\left[\begin{array}{cc}
\ln \left(\frac{Z}{z_{0}}\right)+\ln \left(\frac{8 \varphi_{m}^{4}\left(\frac{Z}{L}\right)}{\left(\varphi_{m}\left(\frac{Z}{L}\right)+1\right)^{2}\left(\varphi_{m}^{2}\left(\frac{Z}{L}\right)+1\right)}\right)-\frac{\pi}{2} \\
+2 \tan ^{-1}\left(\frac{1}{\varphi_{m}\left(\frac{Z}{L}\right)}\right)
\end{array}\right] & L<0 \\
\frac{u_{*}}{\kappa}\left[\ln \left(\frac{Z}{z_{0}}\right)+\varphi_{m}\left(\frac{Z}{L}\right)-1\right] & L>0
\end{array}\right.
$$




$$
T(z)-T_{0}= \begin{cases}\frac{\theta_{*}}{\kappa}\left[\ln \left(\frac{z}{z_{0}}\right)-2 \ln \left(\frac{1}{2}\left(1+\varphi_{m}^{-2}\left(\frac{z}{L}\right)\right)\right)\right]-\frac{g}{c_{p}}\left(z-z_{0}\right) & L<0 \\ \frac{\theta_{*}}{\kappa}\left[\ln \left(\frac{Z}{z_{0}}\right)+\varphi_{m}\left(\frac{z}{L}\right)-1\right]-\frac{g}{c_{p}}\left(z-z_{0}\right) & L>0\end{cases}
$$

The vertical distributions of $k$ and $\varepsilon$ given by Equations 3-14 and 3-16 were used to specify the inlet turbulence parameters and Equation 3-19 was used to specify the specific dissipation rate $(\omega)$. The pressure is internally calculated from the continuity equation and the density is calculated by the code from the ideal gas law.

The outlet boundary is the vertical $z$ line downstream of the fluid domain that was specified as a "flow split outlet" with a split ratio of one. This means that all the flow is assumed to exit from this boundary. Zero horizontal gradients are imposed here, re-enforcing the horizontal homogeneity requirement. Furthermore this type of boundary allows the pressure and temperature profiles to develop from the inlet conditions, rather than having to impose a pressure and temperature here that could over-constrain the solution.

The top boundary is the $x$ line at the top of the fluid domain where the undisturbed flow conditions were prescribed using the "velocity inlet" boundary condition. This includes the velocity components, the temperature and the turbulence quantities. The values for these quantities for the different thermal stability conditions are given in Table 5-7.

Table 5-7: Flow values specified at top boundary

\begin{tabular}{lccccc}
\hline & {$\left[u_{x}, u_{y}, u_{z}\right](\mathrm{m} / \mathrm{s})$} & $T(\mathrm{~K})$ & $k(\mathrm{~J} / \mathrm{kg})$ & $\varepsilon(\mathrm{J} / \mathrm{kg} . \mathrm{s})$ & $\omega\left(\mathrm{s}^{-1}\right)$ \\
\hline Neutral & {$[14.593,0,0]$} & 293.11 & 1.2699 & 0.000544 & 0.0129 \\
Stable & {$[23.629,0,0]$} & 280.65 & 1.1089 & 0.003838 & 0.1039 \\
Unstable & {$[12.622,0,0]$} & 304.09 & 5.5069 & 0.003368 & 0.0184 \\
\hline
\end{tabular}

The bottom fluid boundary that was specified as a wall had the high $z^{+}$wall treatment imposed. The shear stress specification was selected as no-slip and the thermal specification was achieved by specifying the ground temperature found in Table 5-1. The wall surface specification was defined as rough with a roughness constant equal to $k_{s}$ in Table 5-1.

In addition, the velocity, temperature and turbulence quantities at the outlet of the domain, $5000 \mathrm{~m}$ downstream, could be written to a "XYZ internal table" and used as a new set of inlet conditions to a new simulation. In this way the total fetch of the computational domain could effectively be extended to $10000 \mathrm{~m}$. 


\subsection{Results and Discussion}

The differences between the respective properties that are specified or calculated at the domain inlet for the different thermal stratifications are shown in Figure 5-3. They have been made non-dimensional by using the reference values of velocity, height and pressure, or the values that the quantities have at ground level (as given in Table 5-1). The velocities, shown in Figure 5-3(a), display the expected trends namely: all the profiles are nearly logarithmic close to the ground and then begin to diverge from the logarithmic profile with increasing height. The stable velocity profile becomes more linear with increasing height while the unstable profile becomes more curvilinear.

Figure 5-3(b) shows the turbulent kinetic energy profiles for the different thermal stratifications. It is clear that under unstable conditions the turbulent kinetic energy is predicted to increase with height, while it remains constant for neutral conditions and decreases for stable conditions. This is to be expected as the influence of the turbulent production due to buoyancy increases with height, and it consumes turbulence under stable conditions and produces it for unstable conditions. Figure 5-3(c) shows that the dissipation of turbulent kinetic energy is increased for both stable and unstable conditions relative to the neutral atmosphere, due to the fact that $\varphi_{\varepsilon}$, the parameter that scales $\varepsilon$ for the stratified atmosphere is always positive and greater than one.

The specified vertical temperature profiles are given in Figure 5-3(e) and show the adiabatic lapse rate for neutral conditions, a temperature inversion close to the ground under stable conditions and the near ground superadiabatic layer formed under unstable conditions. The density profiles calculated by the code from the ideal gas law are shown in Figure 5-3(d) and reveal that the density decreases with height, except close to the ground under unstable conditions that show an increase in density due to the strong temperature gradient, which, as discussed in the previous chapter, can refract light strongly and lead to the formation of mirages. Figure 5-3(d) also seems to suggest that the densities are higher under unstable conditions. This is, however, misleading and a result of the profile scaling, as the temperatures under unstable conditions are typically much higher than when the atmosphere is neutral or stable, resulting in correspondingly lower densities.

The pressure profiles that are allowed to develop at the inlet are shown in Figure 5-3(f). They show the linear decrease with height expected from a hydrostatic pressure field. The effect of the different densities and temperatures of the air is reflected in the gradient of the profiles, which also reflect the difference in the weight of the fluid. The temperatures under unstable conditions are typically higher and result in lower densities and therefore a lower vertical pressure gradient. The reverse is true for stable conditions, where the densities are higher and the fluid is heavier, resulting in a larger vertical pressure gradient. 
(a)
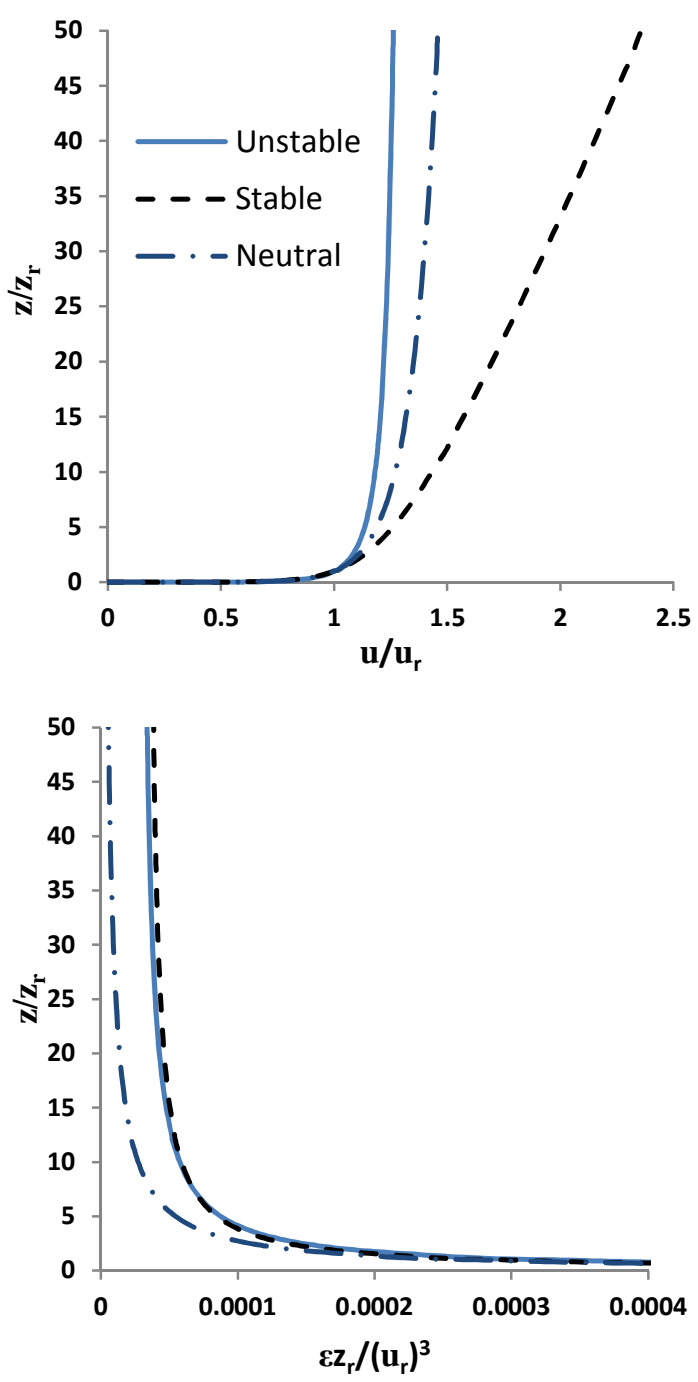

(c)

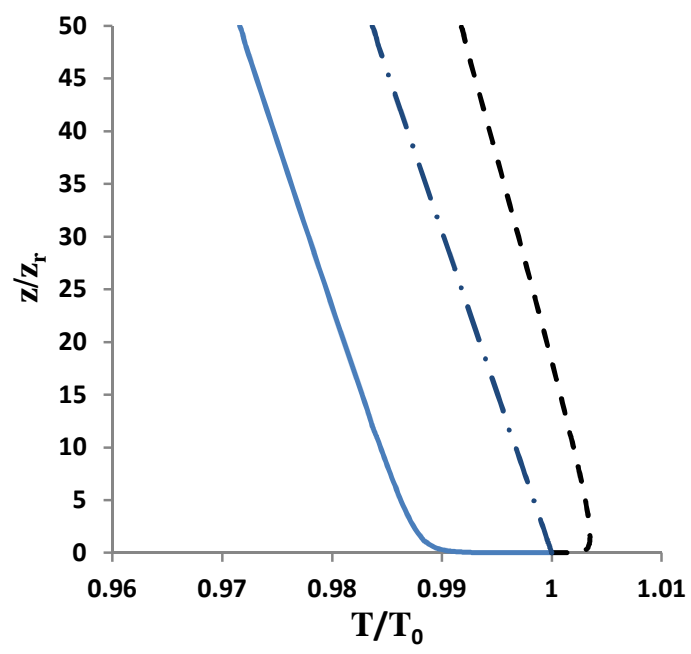

(b)
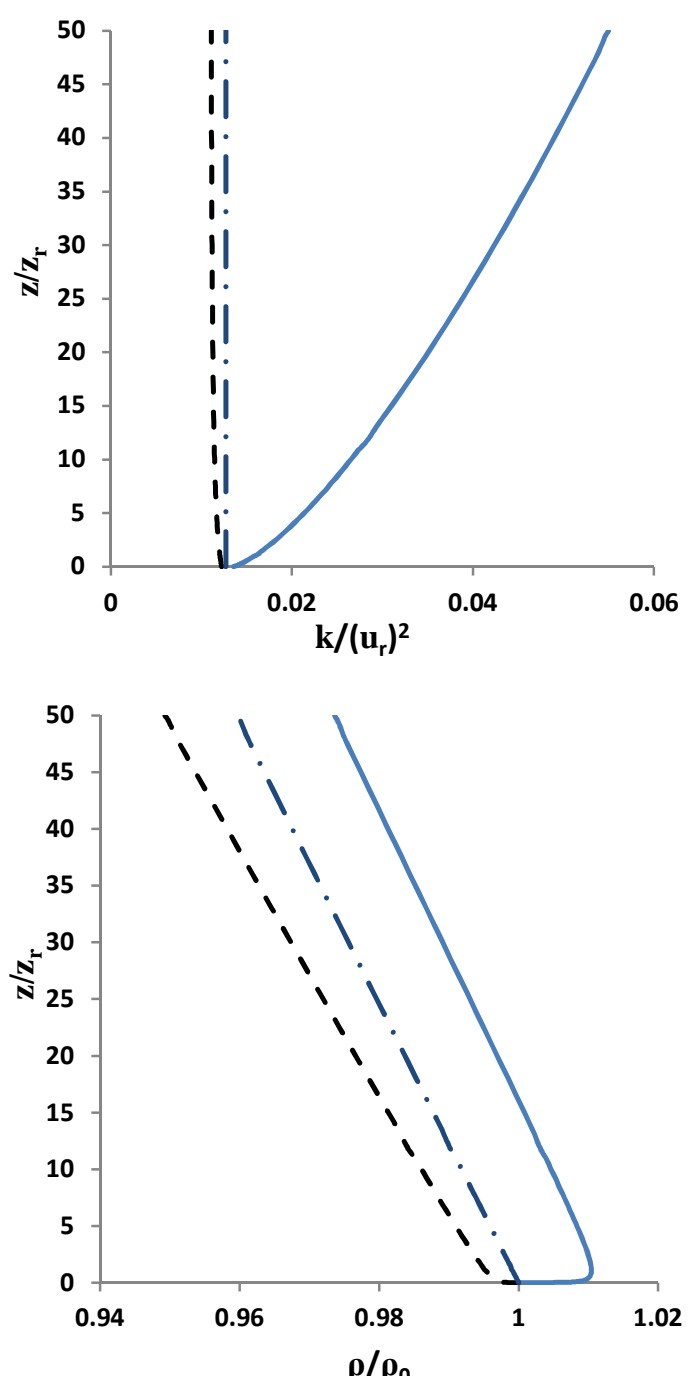

(d)

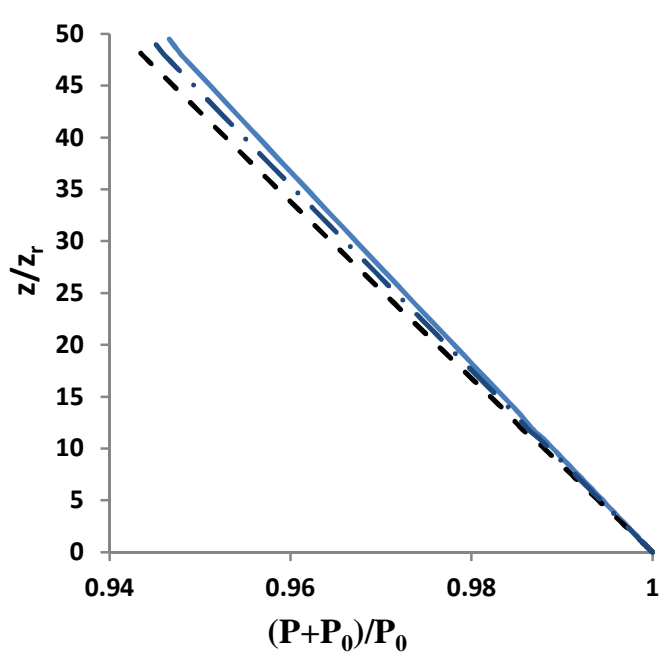

(f)

Figure 5-3: Scaled profiles for flow properties at the domain inlet: (a) velocity; (b) turbulent kinetic energy; (c) turbulent kinetic energy dissipation rate; (d) density; (e) temperature and; (f) pressure. 
The streamwise homogeneity of the velocity and turbulence profiles under neutral conditions for the $k-\varepsilon$ turbulence model are shown in Figure 5-4, where the column on the right shows the lower $50 \mathrm{~m}$ of the domain. It is clear that the velocity profile is well maintained throughout a substantial downstream distance. A slight acceleration of the flow is observed close to the ground, which is consistent with the observation of previous studies (Hargreaves and Wright 2007, Blocken et al. 2007b). The turbulence profiles too are well maintained, with the turbulent kinetic energy shown to decay in the downstream direction, more so after the first $1000 \mathrm{~m}$. The turbulent kinetic energy dissipation rate, shown in Figure 5-3(c) also decays in the downstream direction, but this is likely due to the decay observed in the turbulent kinetic energy. The greatest errors in the profiles are seen close to ground, where energy is being removed by shearing.

The temperature, density and pressure (relative to the reference pressure) profiles are shown in Figure 5-5 for neutral conditions modelled with $k-\varepsilon$ turbulence. Also shown in the plots are the theoretical values expected for the neutral atmosphere, given by Equations 2-7 and 2-10 for temperature and pressure respectively, with density calculated from the ideal gas law. These profiles calculated by the code for pressure and density correspond well with the theoretical values, with the temperature being the adiabatic lapse rate and specified at the inlet. The temperature profile furthermore is maintained well and thereby too the density. It is seen that the pressure is virtually invariant along the entire length of the domain. This is a good result as the pressure must simply balance the weight of the fluid and lead to density variation, while the flow is driven by the shear induced at the top boundary by specifying a horizontal velocity there. This velocity has the effect of feeding momentum into the flow and driving it along. There is consequently no need for a large pressure gradient to develop between the inlet and the outlet of the domain, and the pressure profile is therefore completely dominated by the hydrostatic effect.

The same results for the neutral atmosphere and the $k$ - $\omega$ turbulence model are shown in Figure 5-6 and Figure 5-7. The first thing to notice is that the horizontal homogeneity errors are more pronounced when compared to the $k$ - $\varepsilon$ turbulence model, with the profile for the turbulent kinetic energy, shown in Figure 5-6(b), showing much more substantial decay in the downwind direction. There is also slightly more acceleration of the velocity profile close to the ground, but the profile maintenance for temperature, density and pressure are similar for the two turbulence models. These results, although slightly worse than those attained for the $\mathrm{k}-\varepsilon$ turbulence model, still show fairly good maintenance of the flow profiles. A comparison of the homogeneity error at a height of $10 \mathrm{~m}$, relative to the inlet conditions for the two turbulence models is shown in Figure 5-8. It illustrates clearly the higher errors made by the $k-\omega$ turbulence model, but shows that the error starts to stabilise after the first $5000 \mathrm{~m}$. It can also be seen that the temperature profile is almost equally well maintained by both turbulence models. 

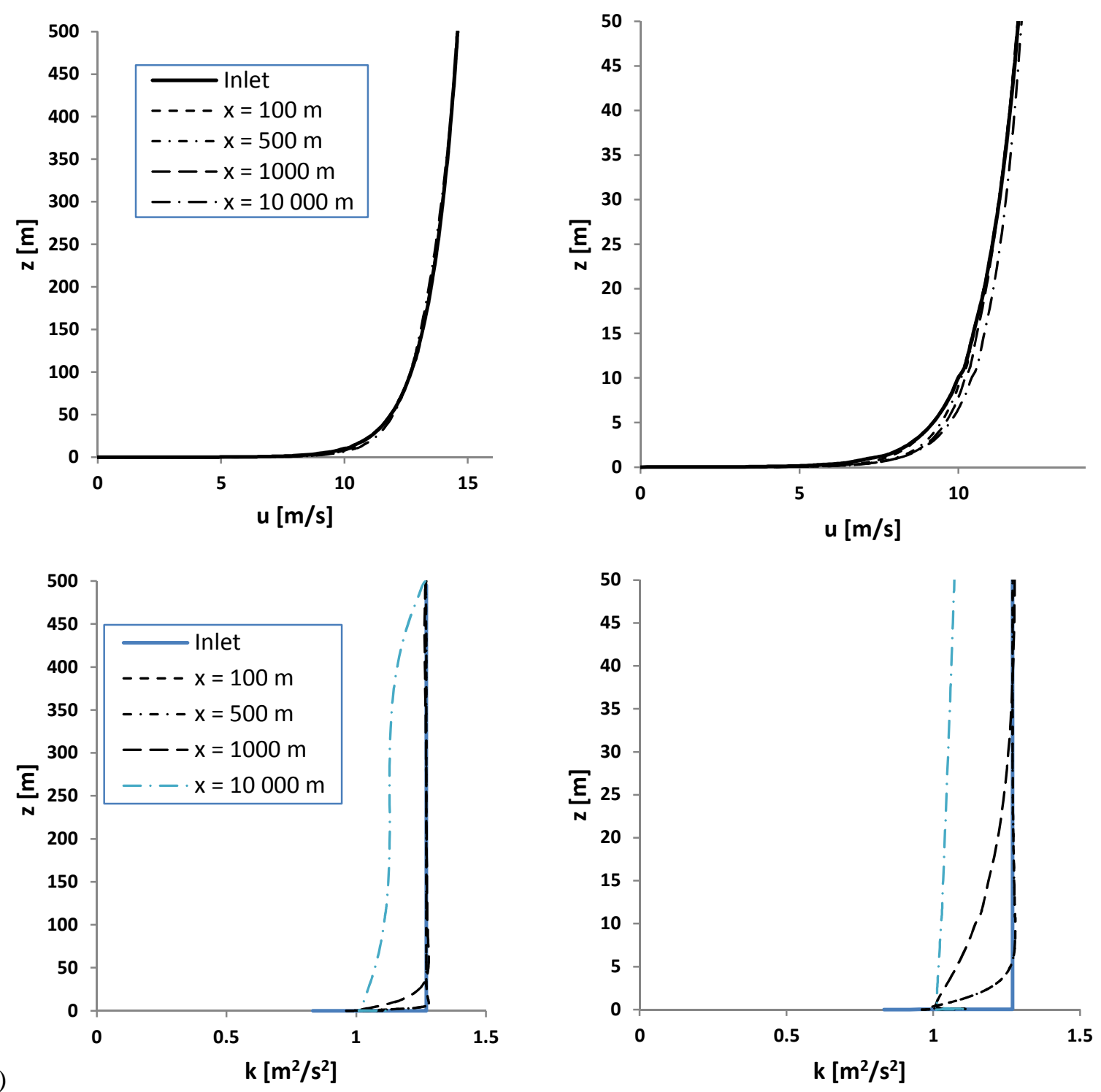

(b)
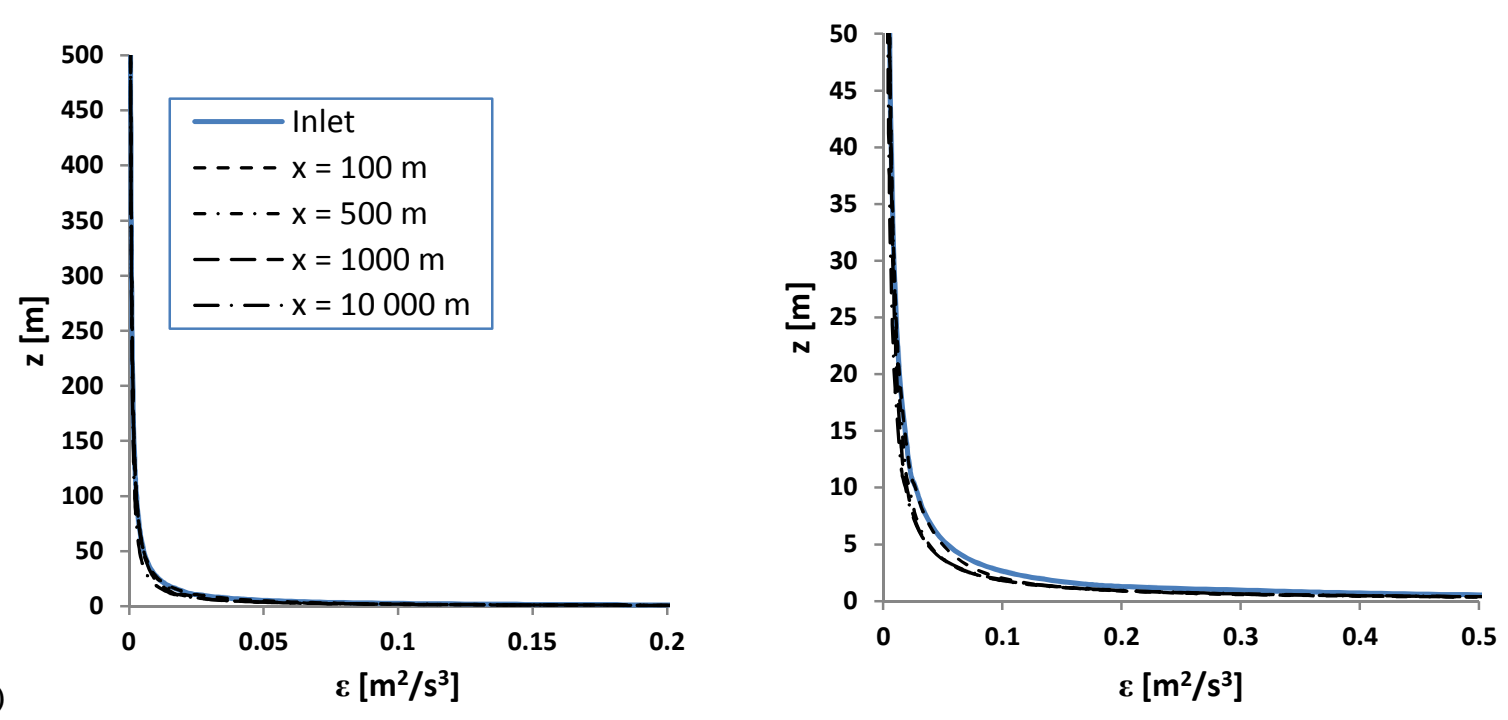

Figure 5-4: Neutral CFD $k-\varepsilon$ model results illustrating streamwise gradients for (a) wind speed $u$; (b) turbulent kinetic energy $k$; and (c) turbulent dissipation rate $\varepsilon$. The right hand column shows the lowest $50 \mathrm{~m}$ of the domain. 


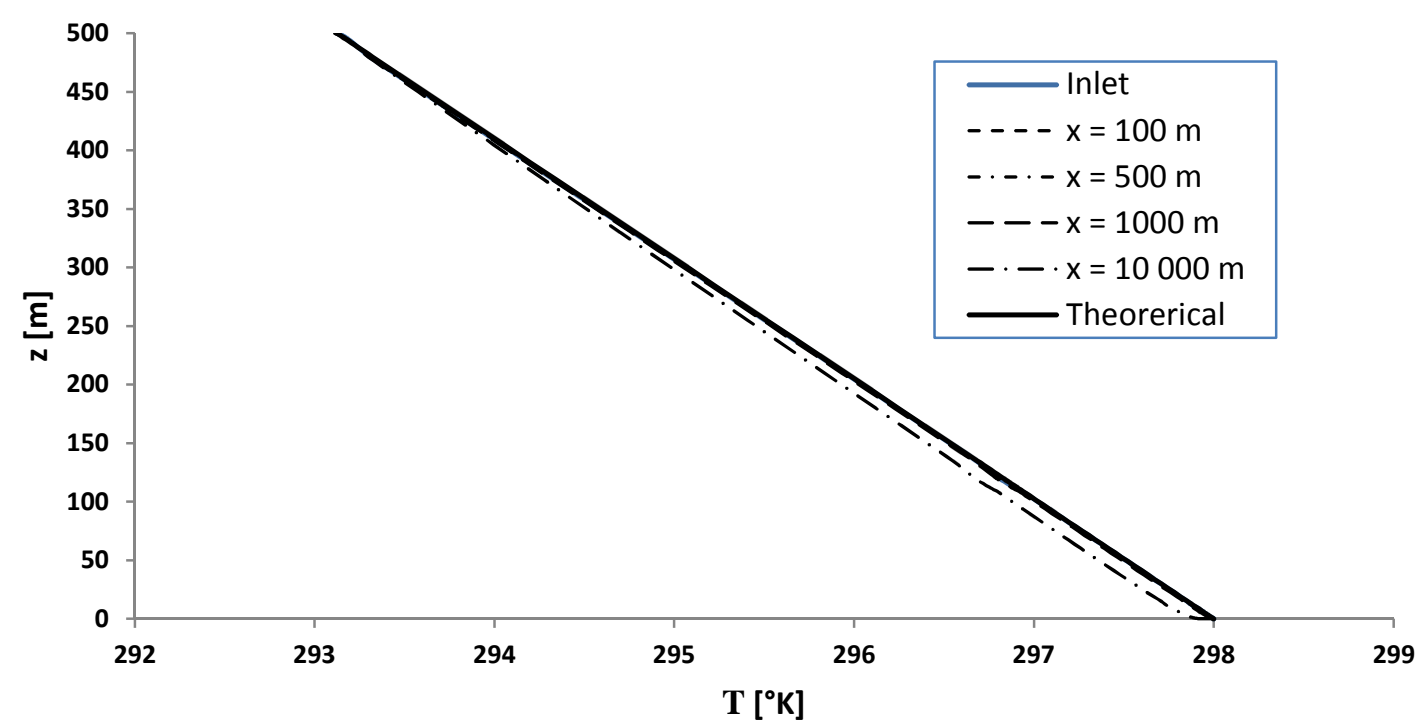

(a)

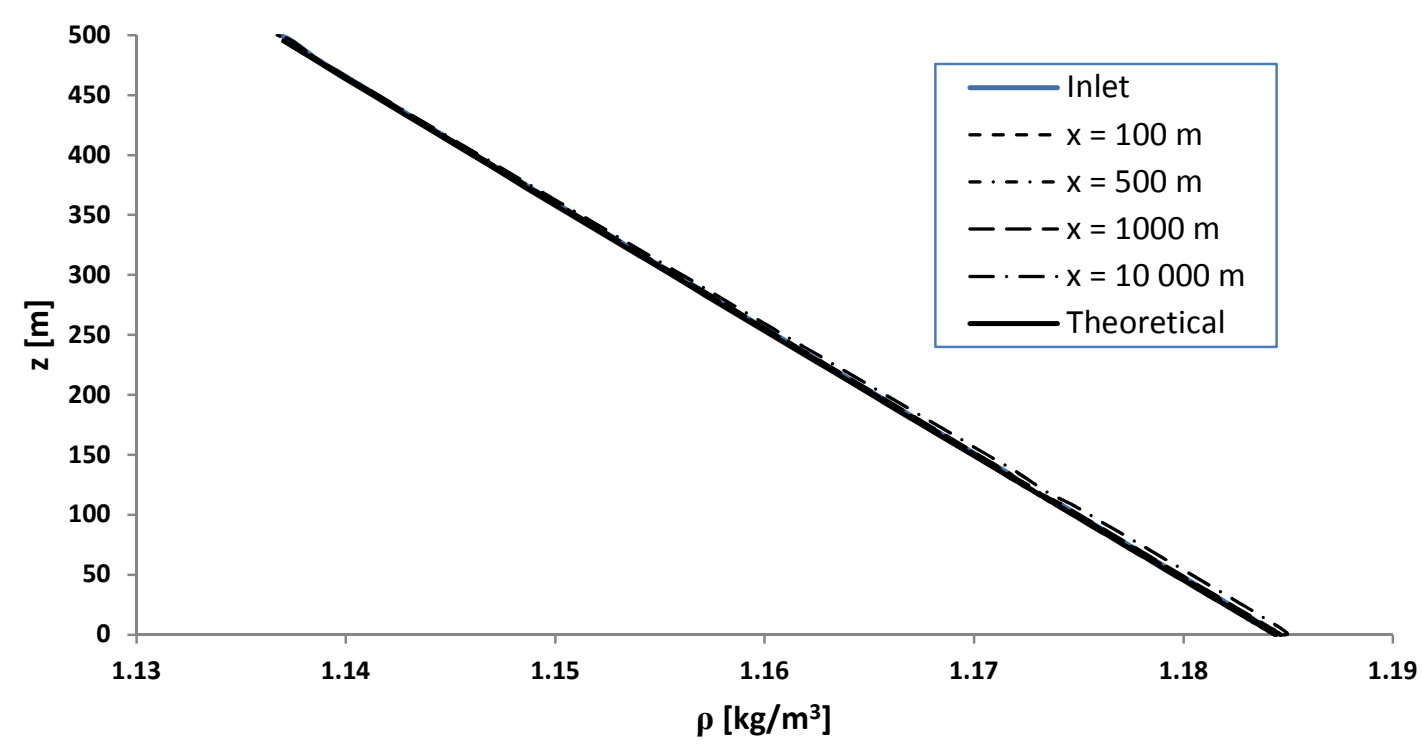

(b)

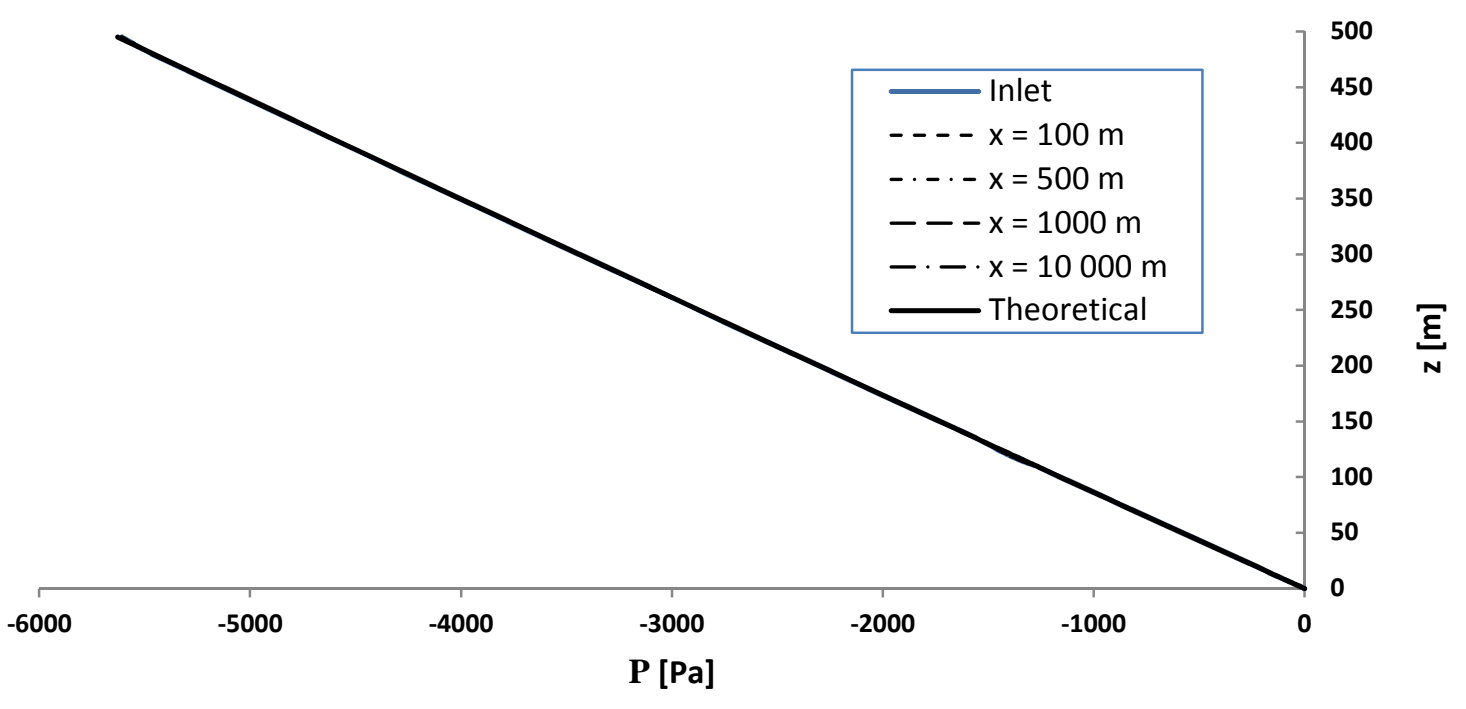

Figure 5-5: Neutral CFD $k-\varepsilon$ model results illustrating streamwise gradients for (a) temperature $T$; (b) density $\rho$; and (c) pressure $P$. Theoretical values for the neutral atmosphere also shown. 

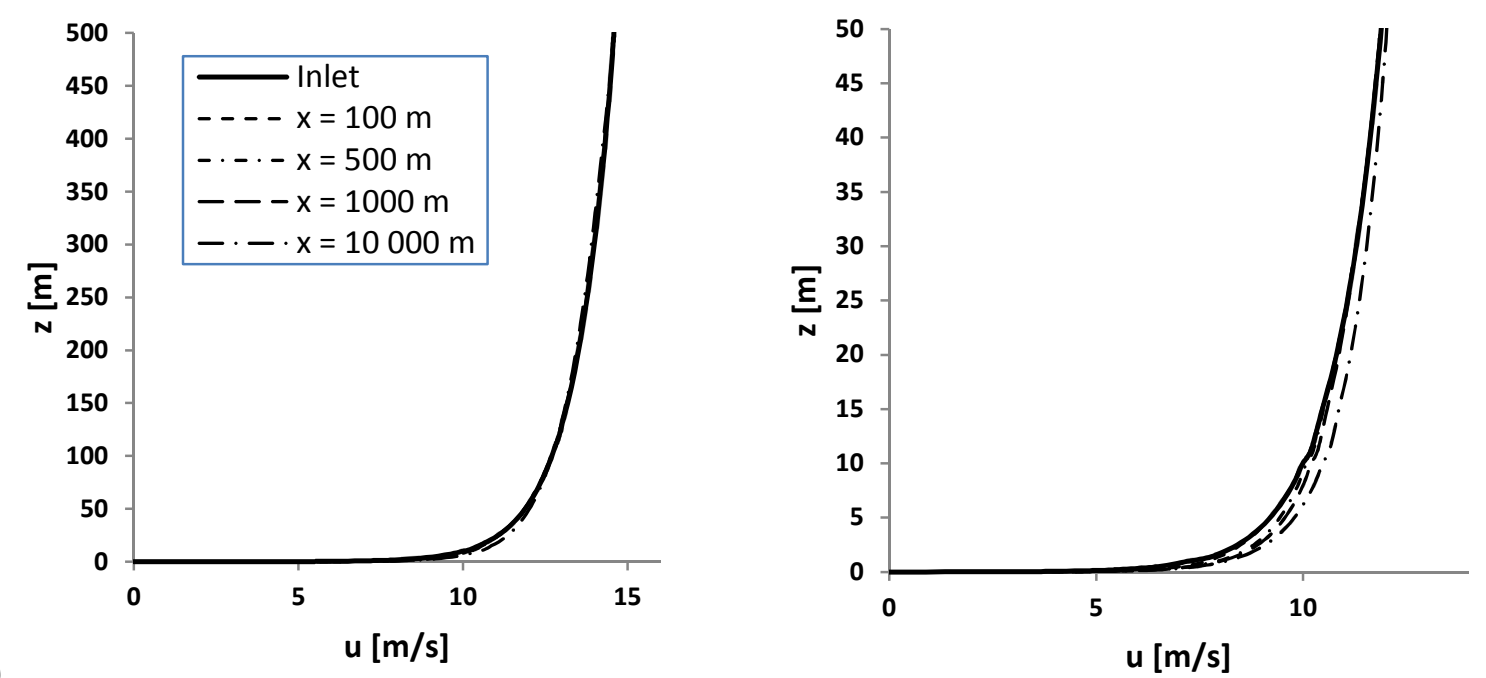

(a)
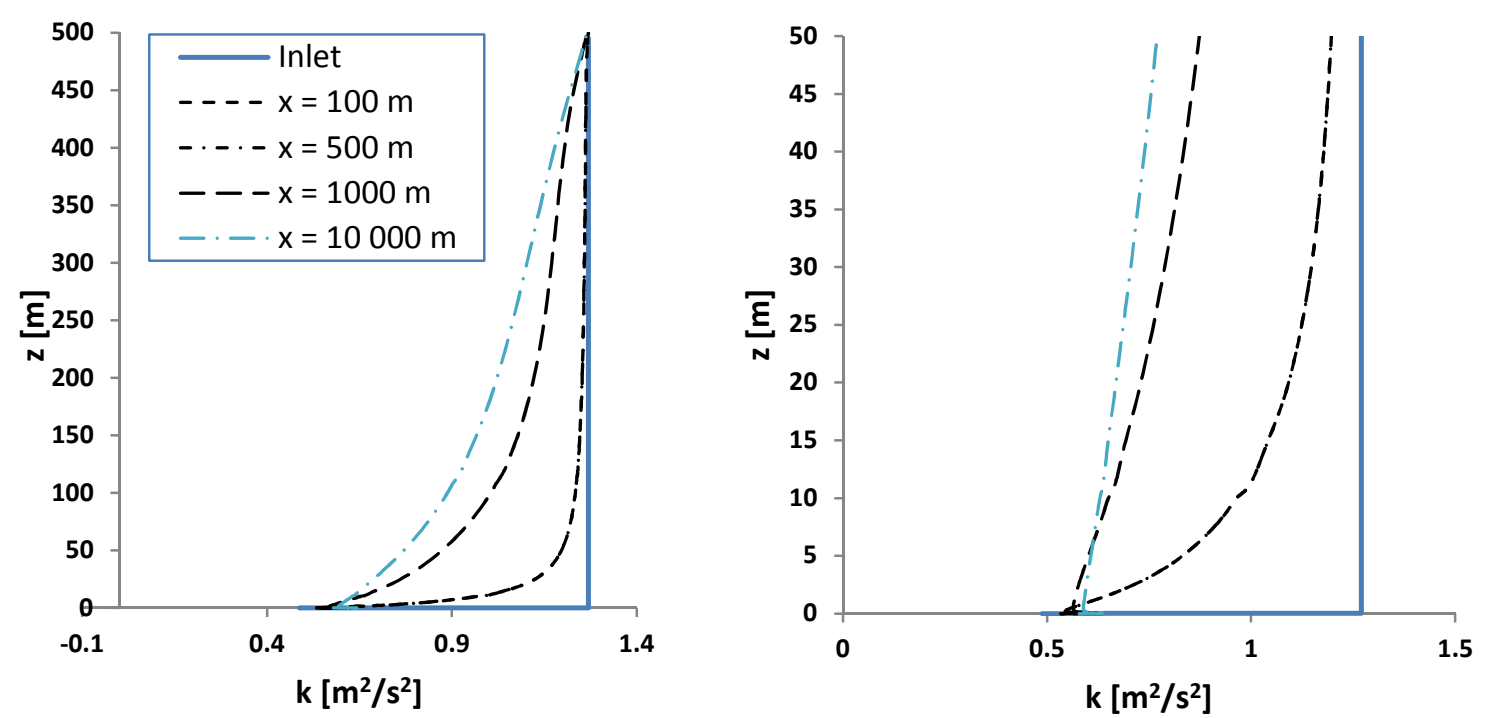

(b)
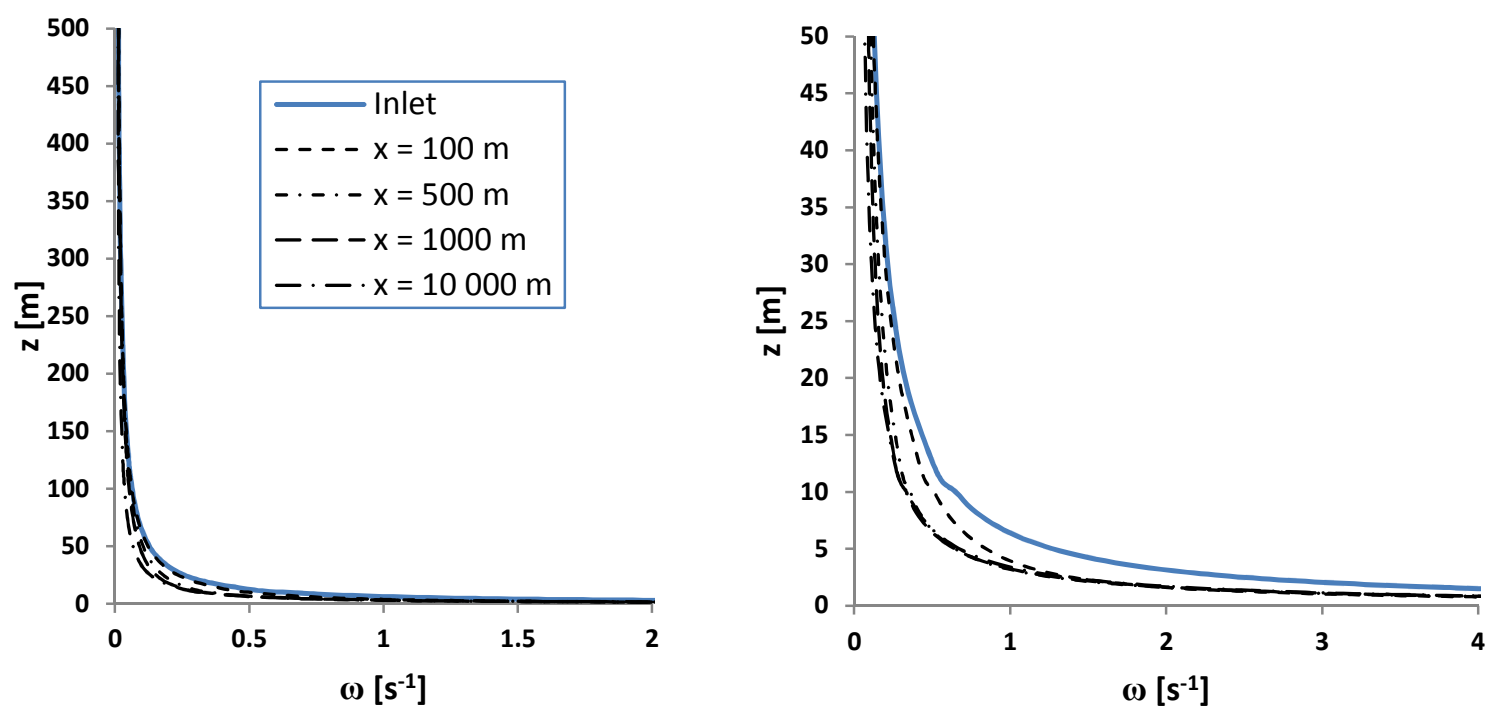

(c)

Figure 5-6: Neutral CFD $k-\omega$ model results illustrating streamwise gradients for (a) wind speed $u$; (b) turbulent kinetic energy $k$; and (c) specific dissipation rate $\omega$. The right hand column shows the lowest $50 \mathrm{~m}$ of the domain. 


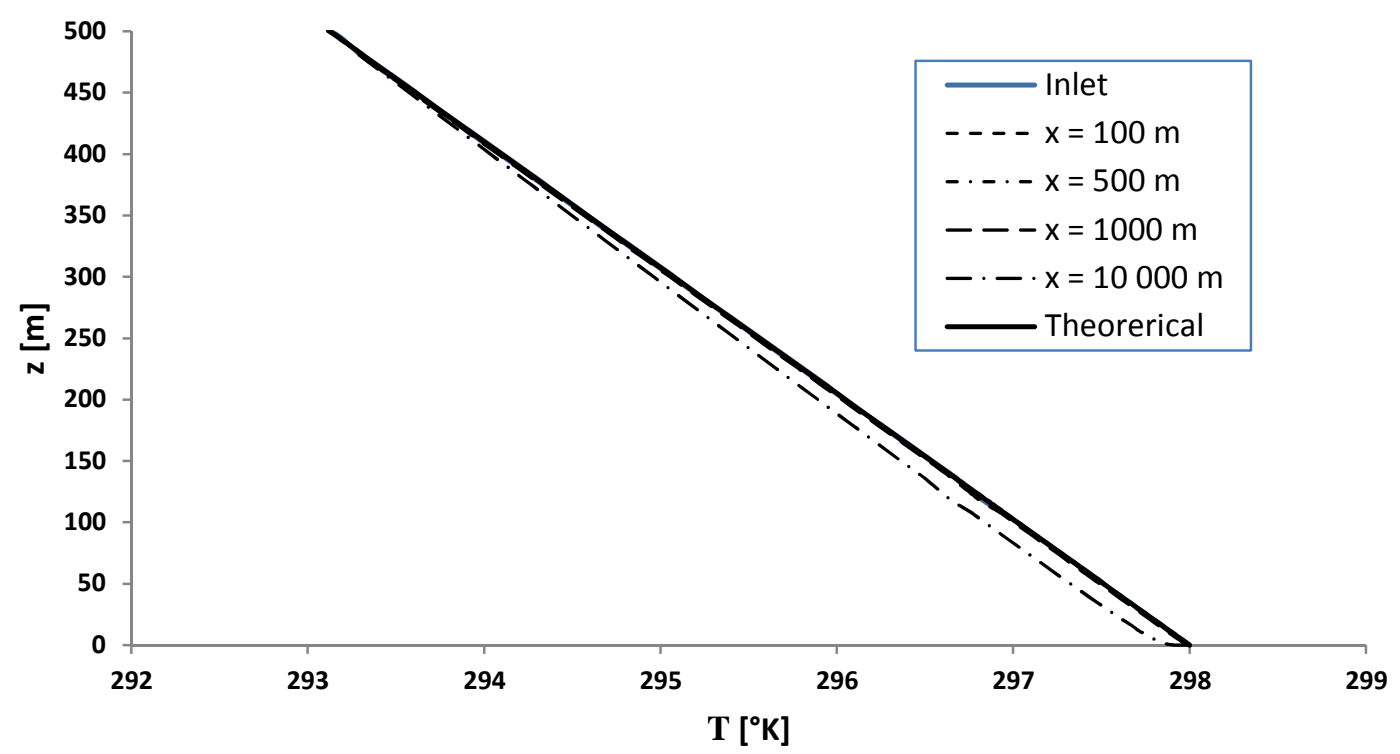

(a)

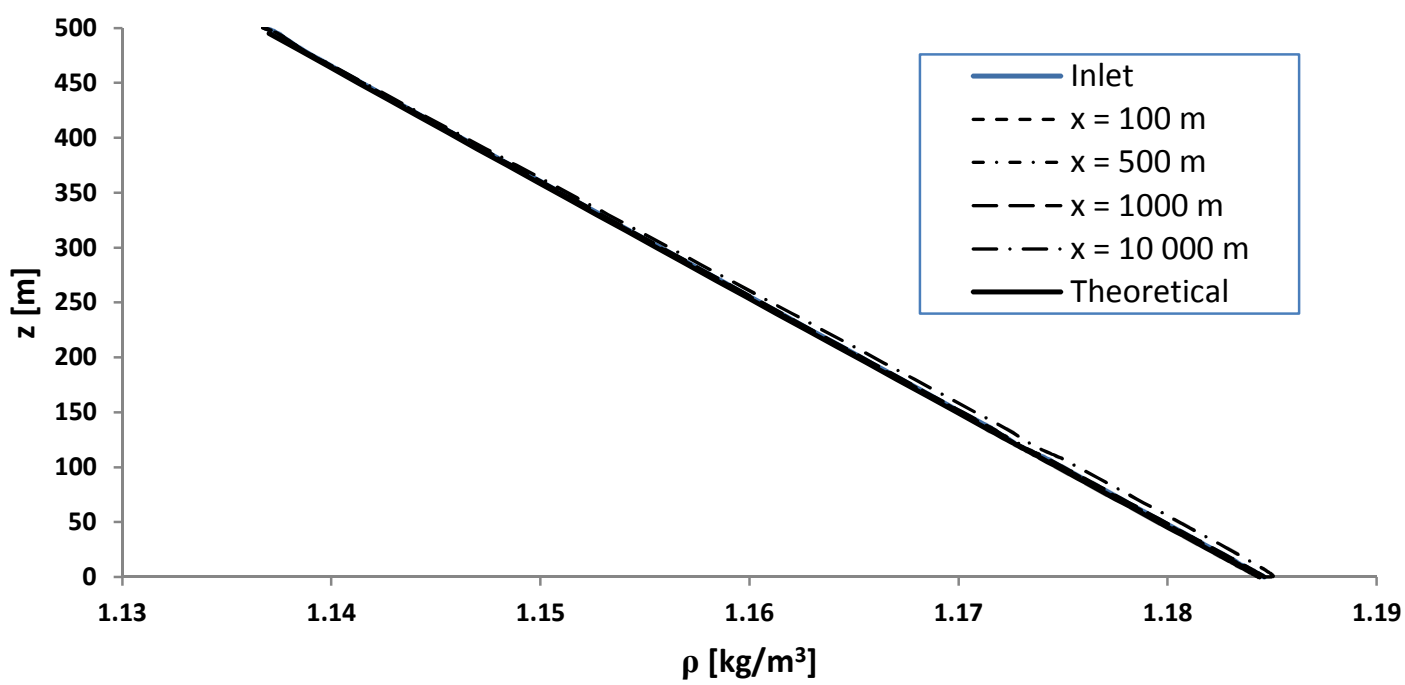

(b)

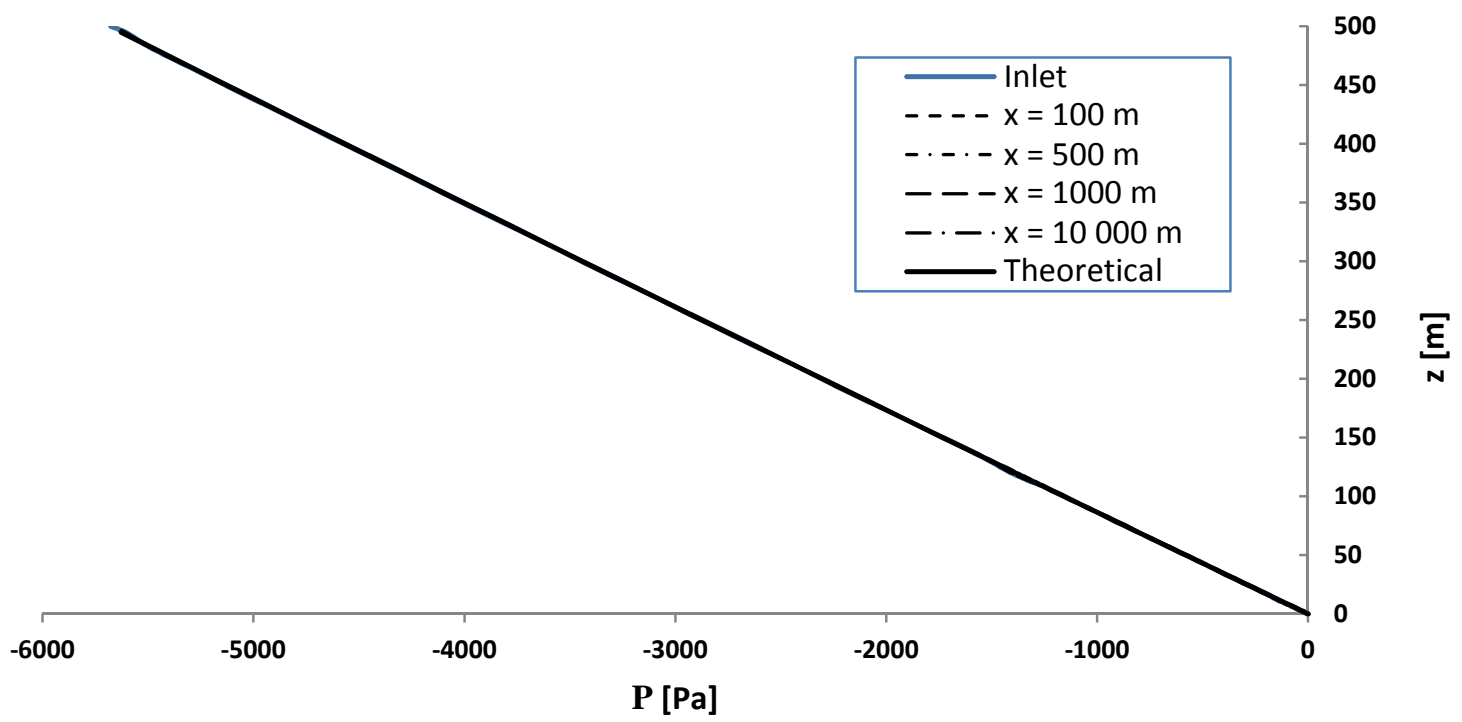

(c)

Figure 5-7: Neutral CFD $k-\omega$ model results illustrating streamwise gradients for (a) temperature $T$; (b) density $\rho$; and (c) pressure $P$. Theoretical values for the neutral atmosphere also shown. 


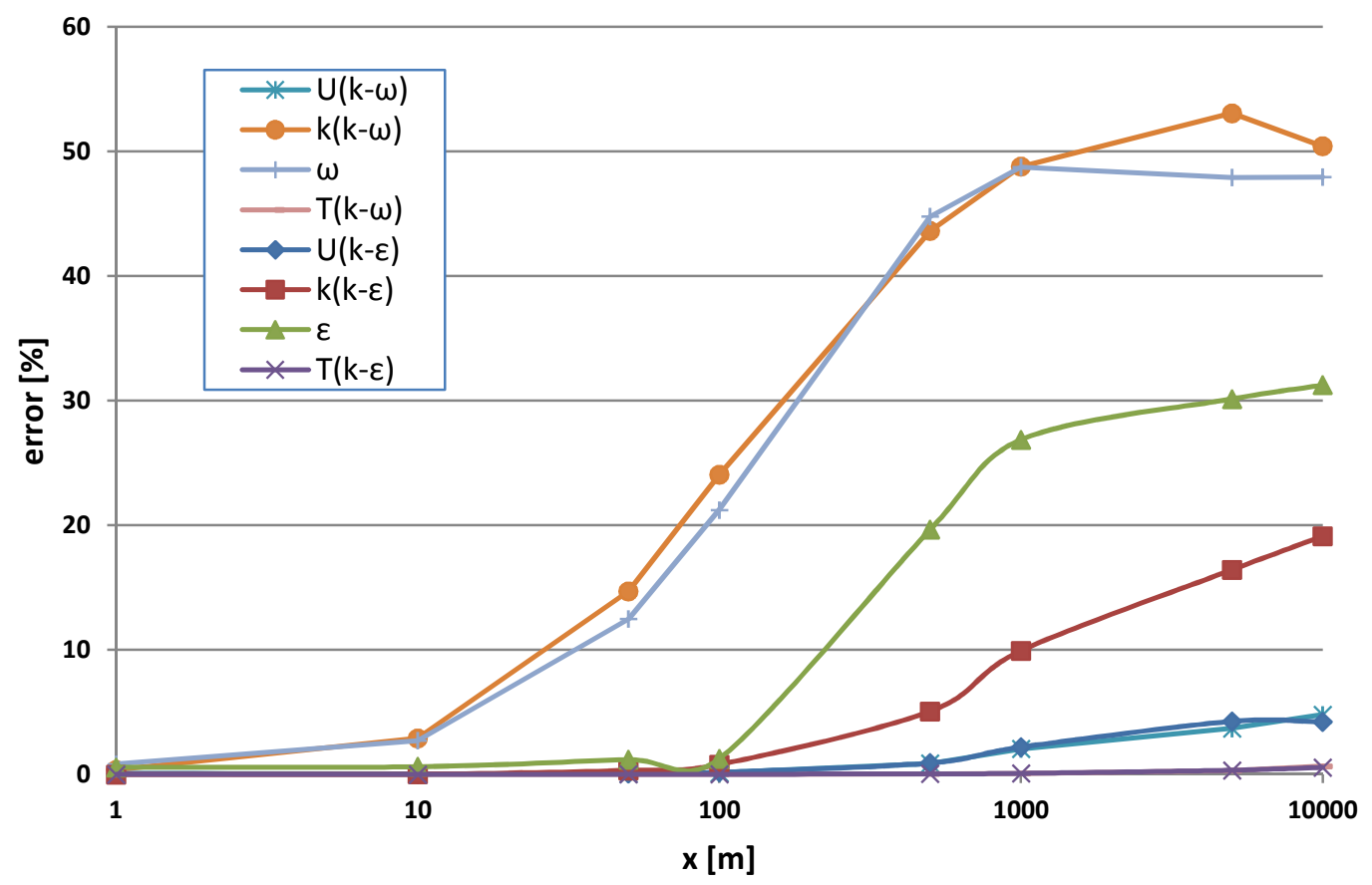

Figure 5-8: Relative change of the flow parameters for the $k-\varepsilon$ and $k-\omega$ turbulence models relative to the inlet for neutral conditions.

Inspection of Figure 5-4(b) and Figure 5-6(b) reveal that in both of the turbulence models the $k$-value is under predicted at the first cell centroid above the wall. The subsequent decay of the profile extends from this point up in the vertical direction, seen clearly in Figure 5-6(b) for the $k$ - $\omega$ turbulence model. This phenomenon will be shown to occur consistently for the subsequent simulations of the stable and unstable atmosphere too, and suggests an issue with the wall function approach.

The results of the homogeneity error in the flow properties for the simulation of the stable atmosphere using the $k-\varepsilon$ turbulence model are shown in Figure 5-9 and Figure 5-10. We again observe a slight acceleration of the flow velocity in the lower part of the domain and decay in the turbulent kinetic energy profile. Similar to the $k$-profile error observed for the neutral conditions, we see that the turbulent kinetic energy is under predicted in the first node above the wall. The temperature, density and pressure profiles, however, are well maintained. The $k$ - $\omega$ turbulence model profiles for the stable atmosphere, which are given in Appendix F, reveal that the profiles are less well maintained than those found for the $k$ - $\varepsilon$ turbulence model, as was shown for the neutral conditions. The inhomogeneity error at a height of $10 \mathrm{~m}$ for the two turbulence models for the stable atmosphere relative to the inlet profiles are shown in Figure 5-11. The errors are of similar size to those found for neutral conditions, and like the neutral conditions the error appears to stabilise after about $5000 \mathrm{~m}$. The profiles of the density, temperature and pressure for the $k-\omega$ turbulence model were found to be similar to those shown in Figure 5-10 and are also given in Appendix F. 

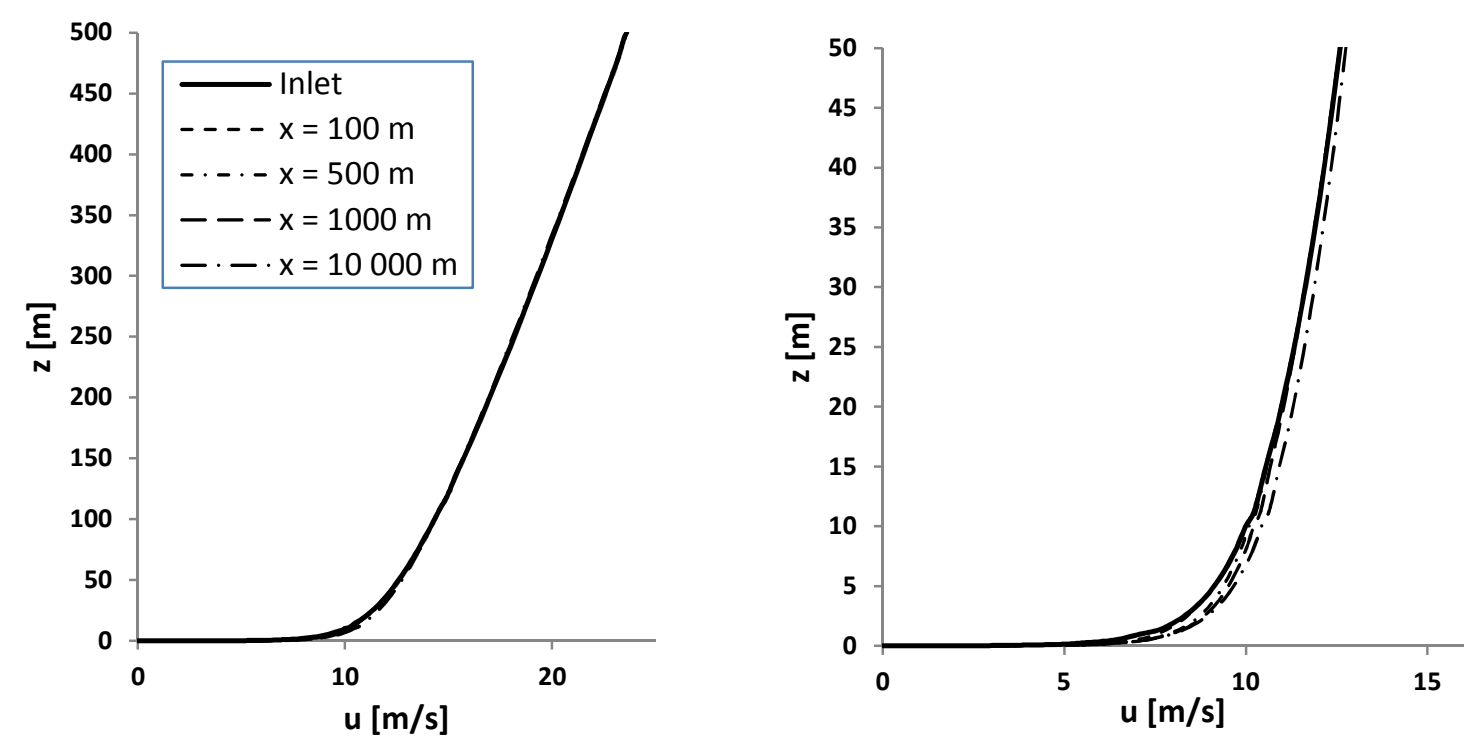

(a)
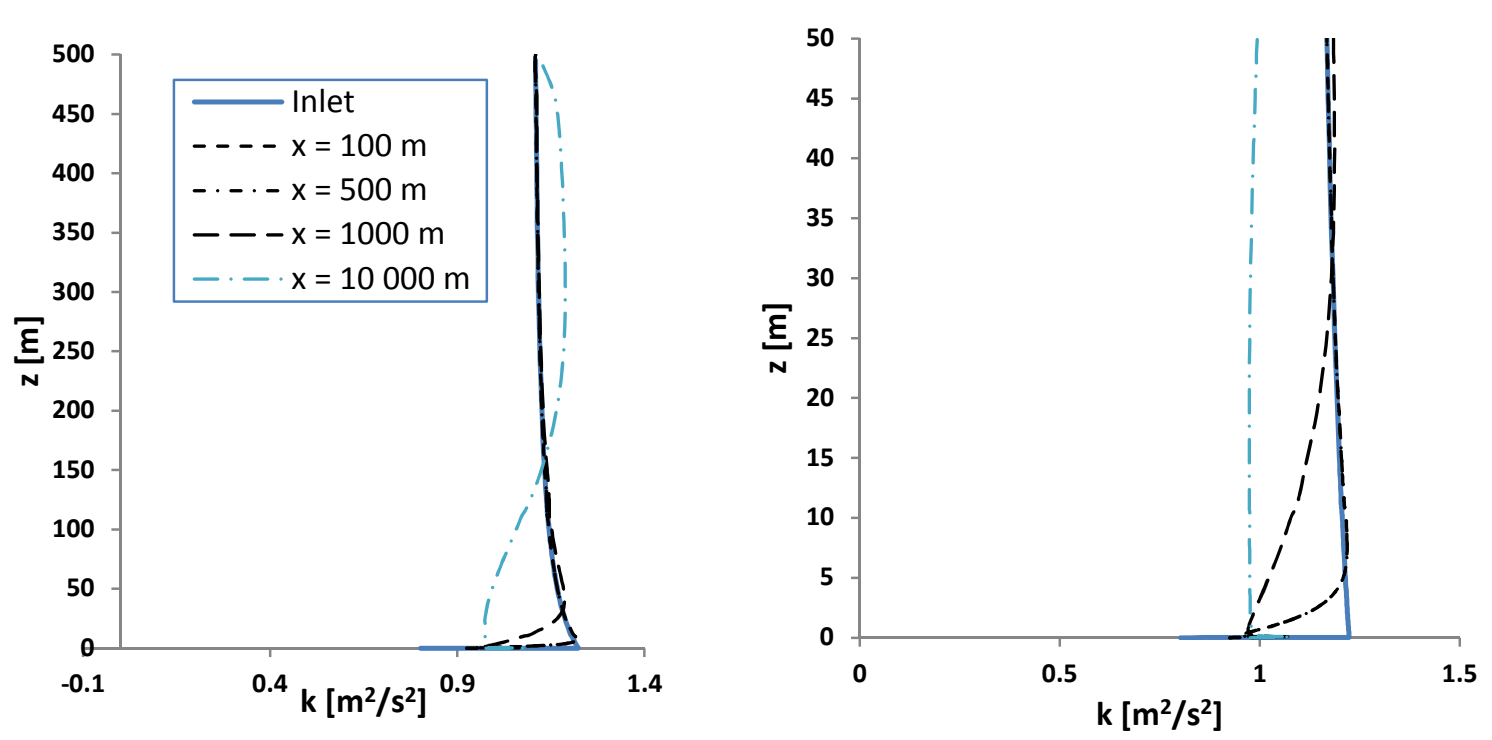

(b)
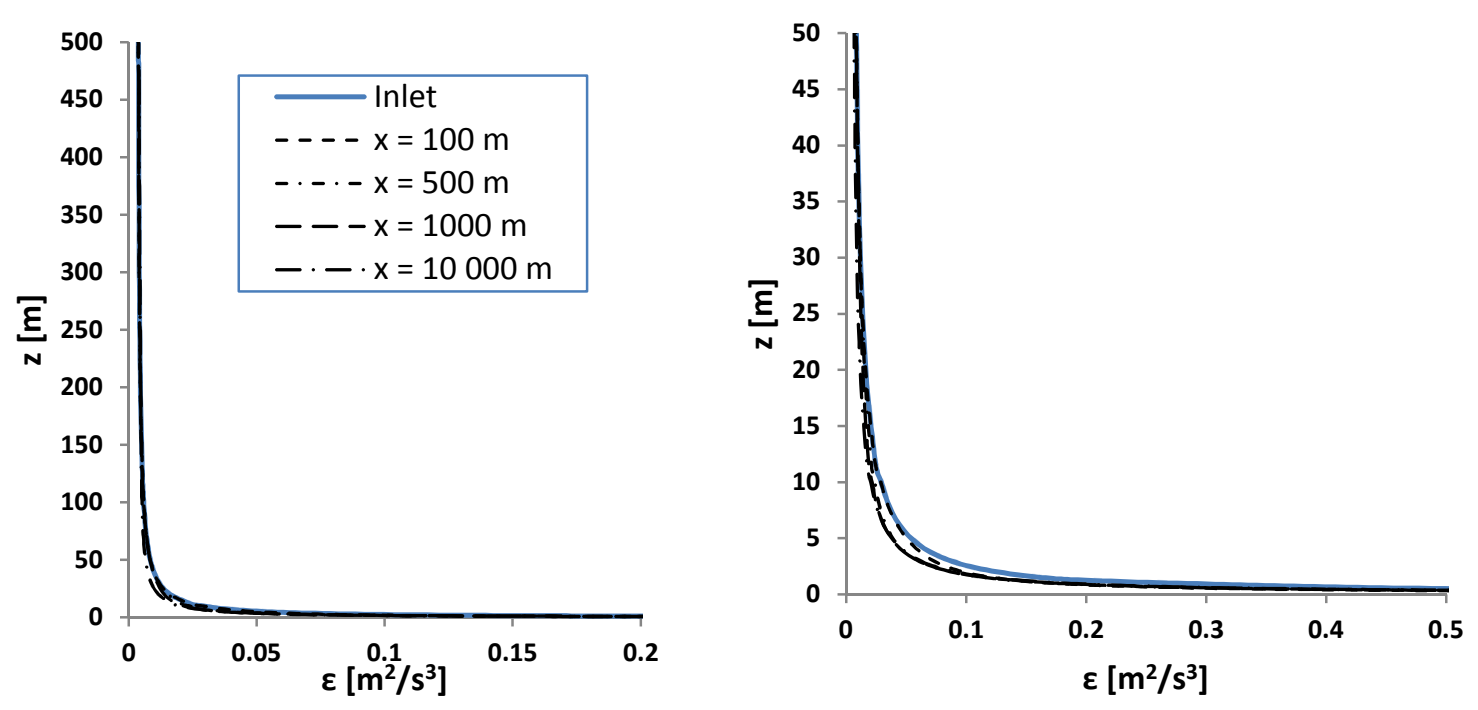

Figure 5-9: Stable CFD $k$ - $\varepsilon$ model results illustrating streamwise gradients for (a) wind speed $u$; (b) turbulent kinetic energy $k$; and (c) turbulent dissipation rate $\varepsilon$. The right hand column shows the lowest $50 \mathrm{~m}$ of the domain. 


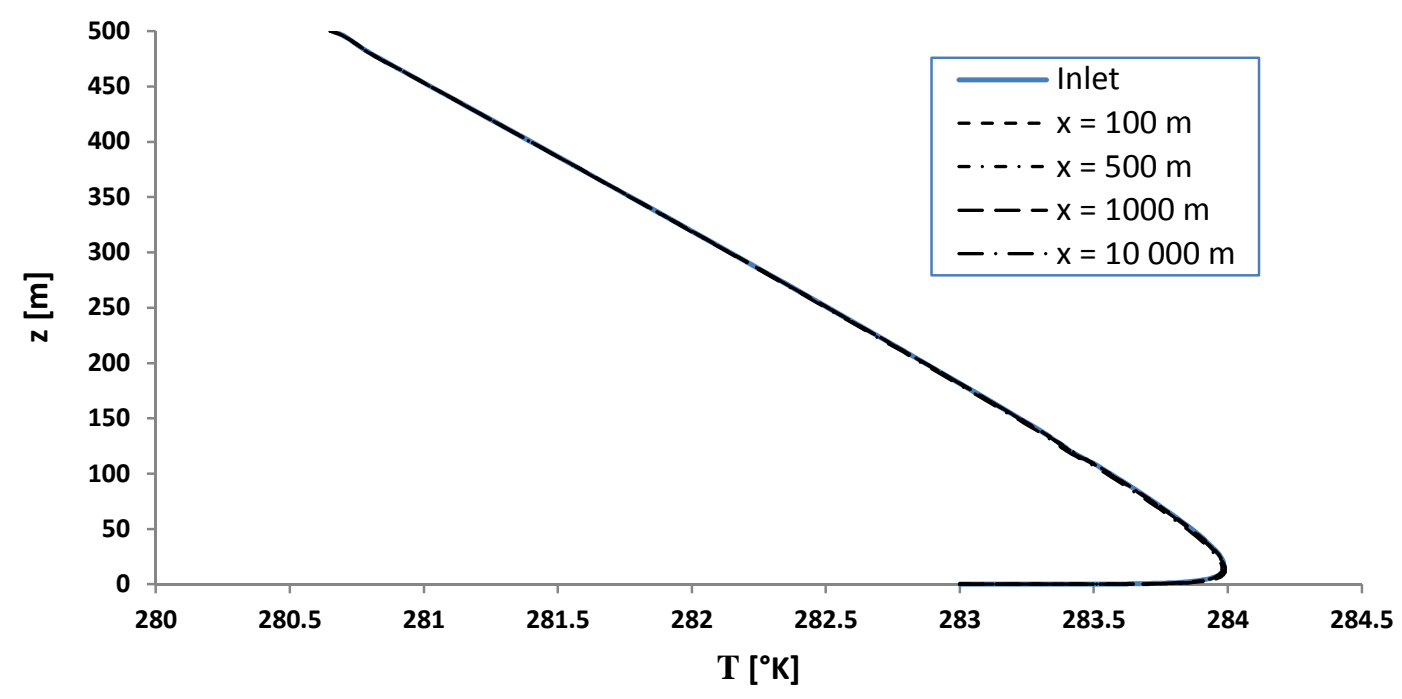

(a)

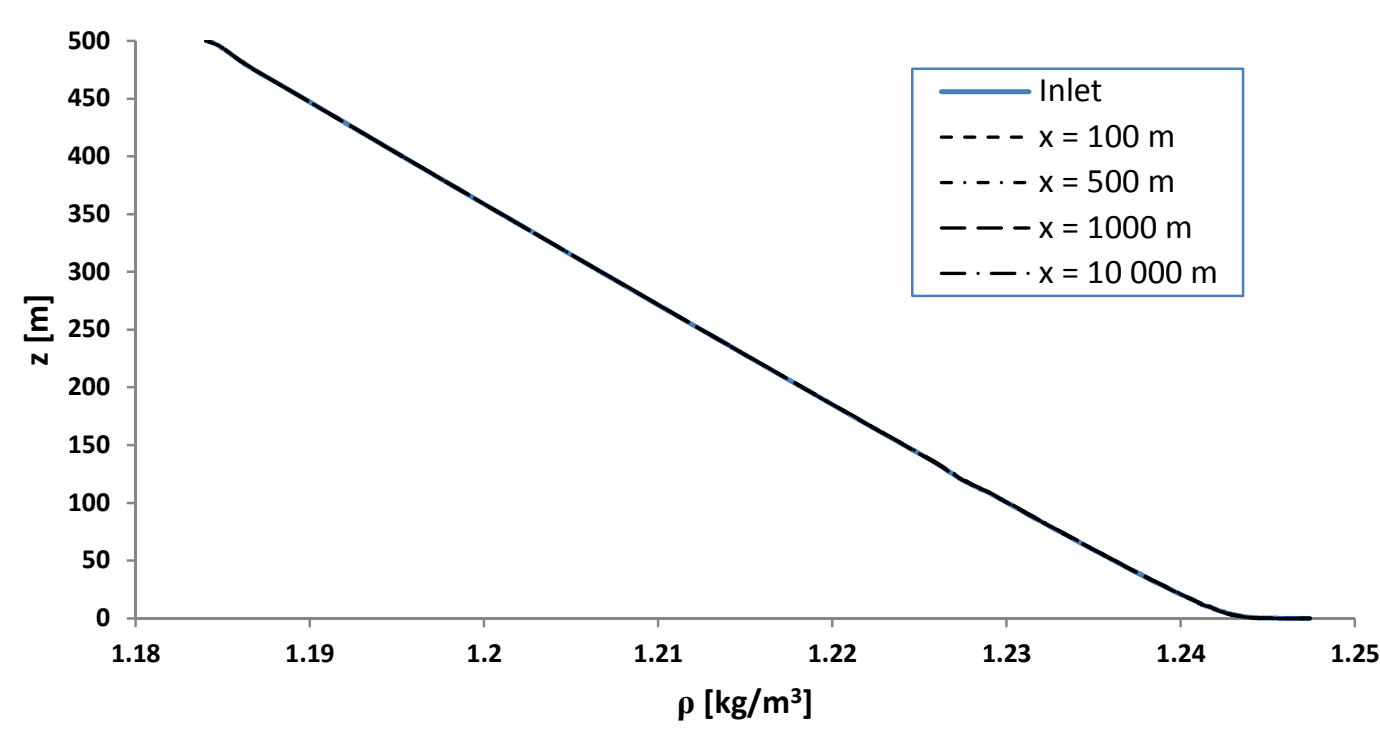

(b)

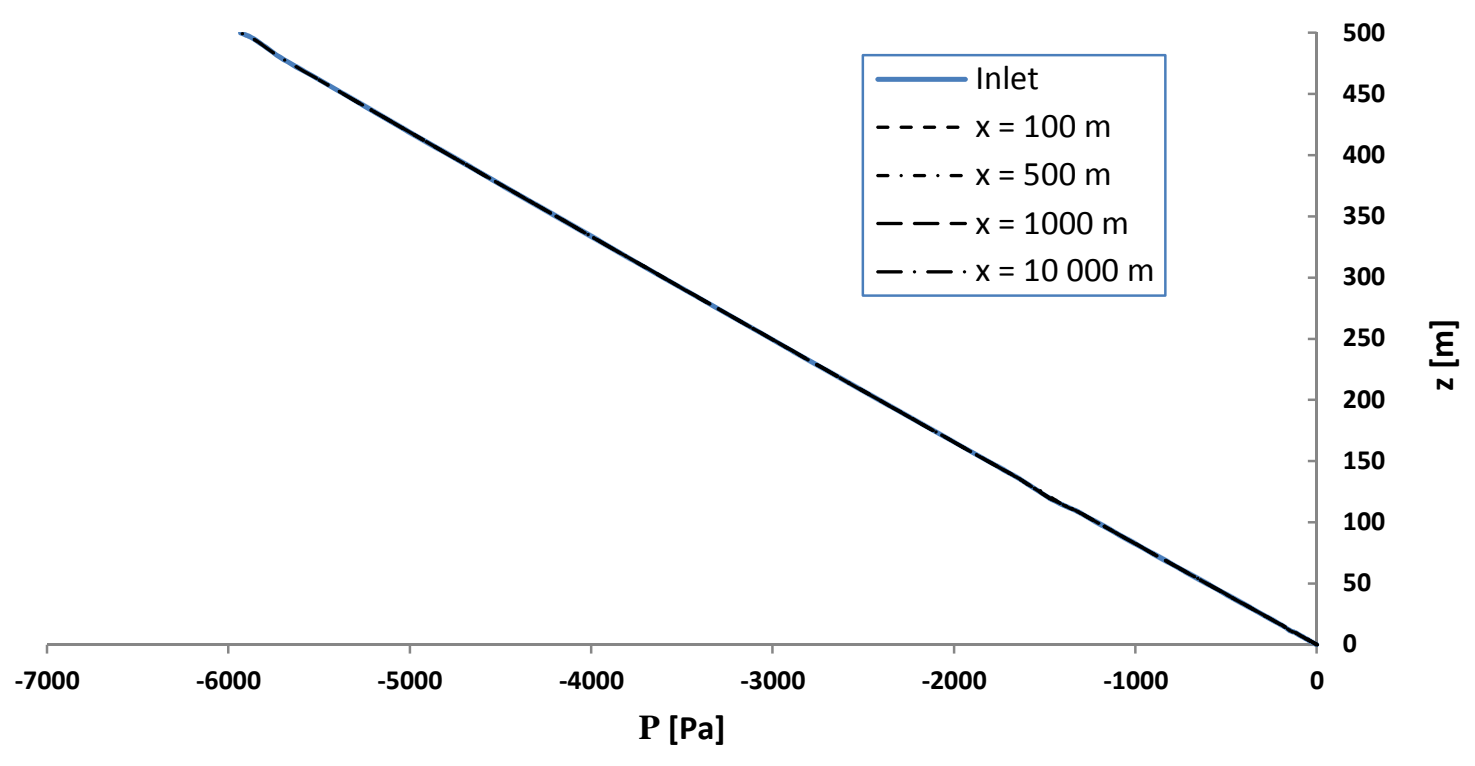

Figure 5-10: Stable CFD $k-\varepsilon$ model results illustrating streamwise gradients for (a) temperature $T$; (b) density $\rho$; and (c) pressure $P$. 


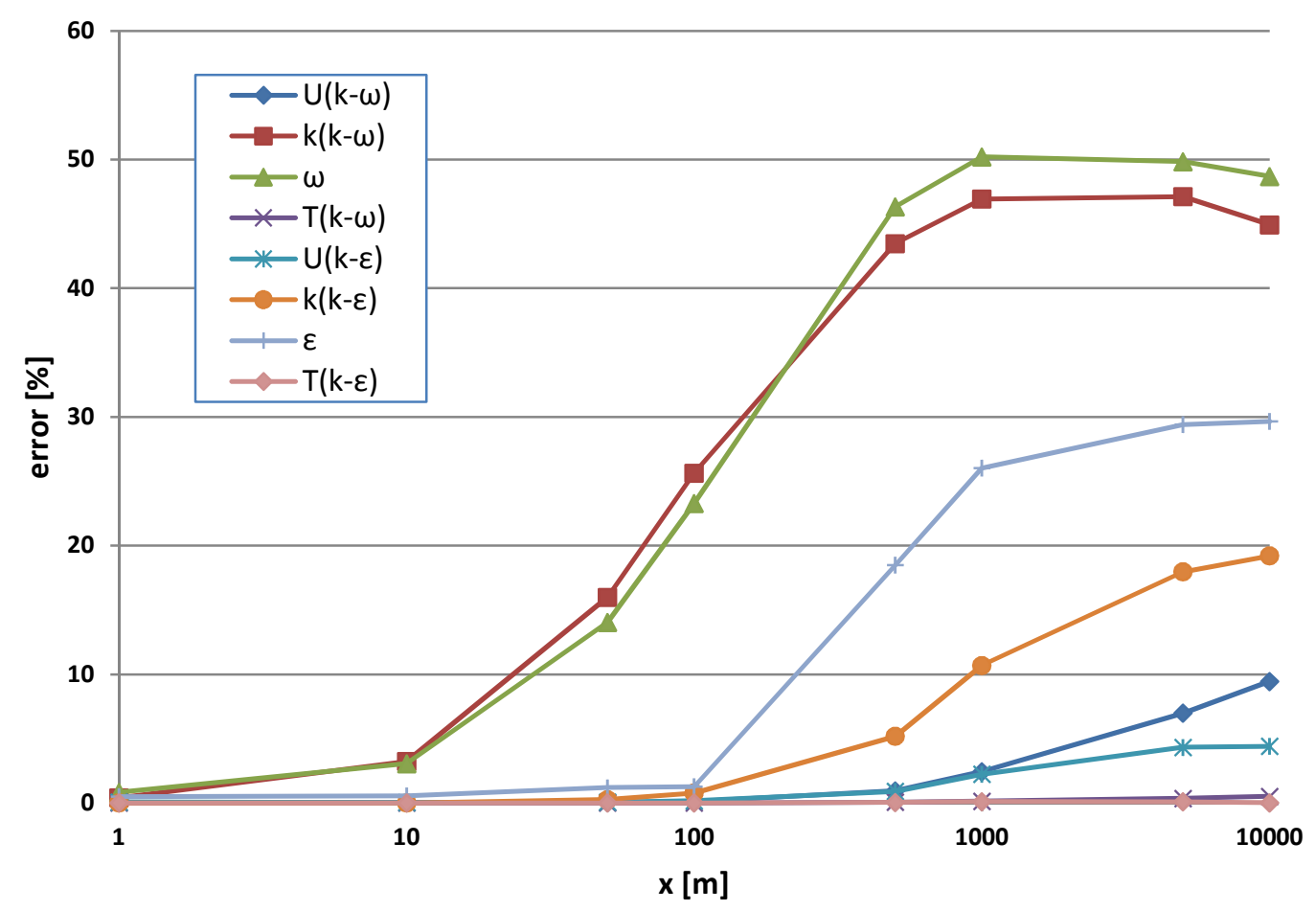

Figure 5-11: Relative change of the flow parameters for the $k-\varepsilon$ and $k-\omega$ turbulence models relative to the inlet for stable conditions.

The results for the analysis of the homogeneity errors in the flow parameters for the unstable atmosphere are shown in Figure 5-12 and Figure 5-13 for the $k-\varepsilon$ turbulence model, with the $k$ - $\omega$ turbulence model results for unstable conditions presented in Appendix F. The results for the unstable atmosphere are qualitatively similar to the results achieved for the neutral and stable atmospheres. The inhomogeneity error at a height of $10 \mathrm{~m}$ for the two turbulence models for the unstable atmosphere once again relative to the inlet profiles are shown in Figure 5-14. Again the $k-\varepsilon$ turbulence model proved better at maintaining the turbulence parameter profiles than the $k-\omega$ turbulence model, with the error for the unstable case being of a similar size to those made for the neutral and stable atmospheres.

The homogeneity errors for the neutral, stable and unstable atmospheres were consistently similar, and therefore reveal that these profiles were equally well maintained along the fetch of the domain. In addition, full variation in density, temperature and pressure was allowed, and the results correspond well with the results predicted for the neutral atmosphere from the theory. In all cases good convergence of the solution was found and therefore minimal numerical instability was encountered. The fact that the pressure was found to vary very little along the length of the fluid domain for all the thermal stratifications is consistent with the assumptions of the horizontally homogenous ABL, with the flow instead being driven by the velocity specified at the top boundary, and therefore by shear. 
(a)
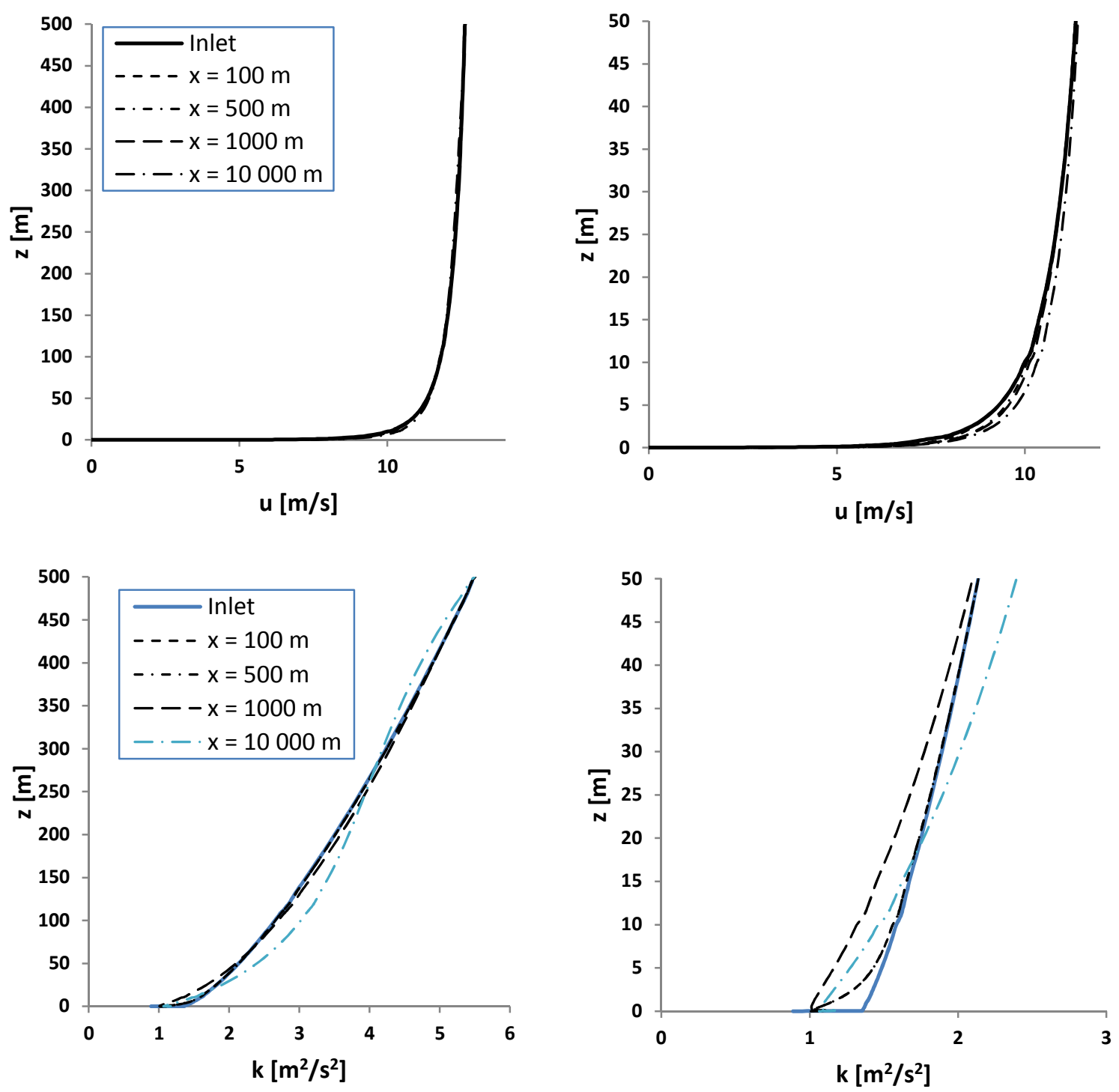

(b)
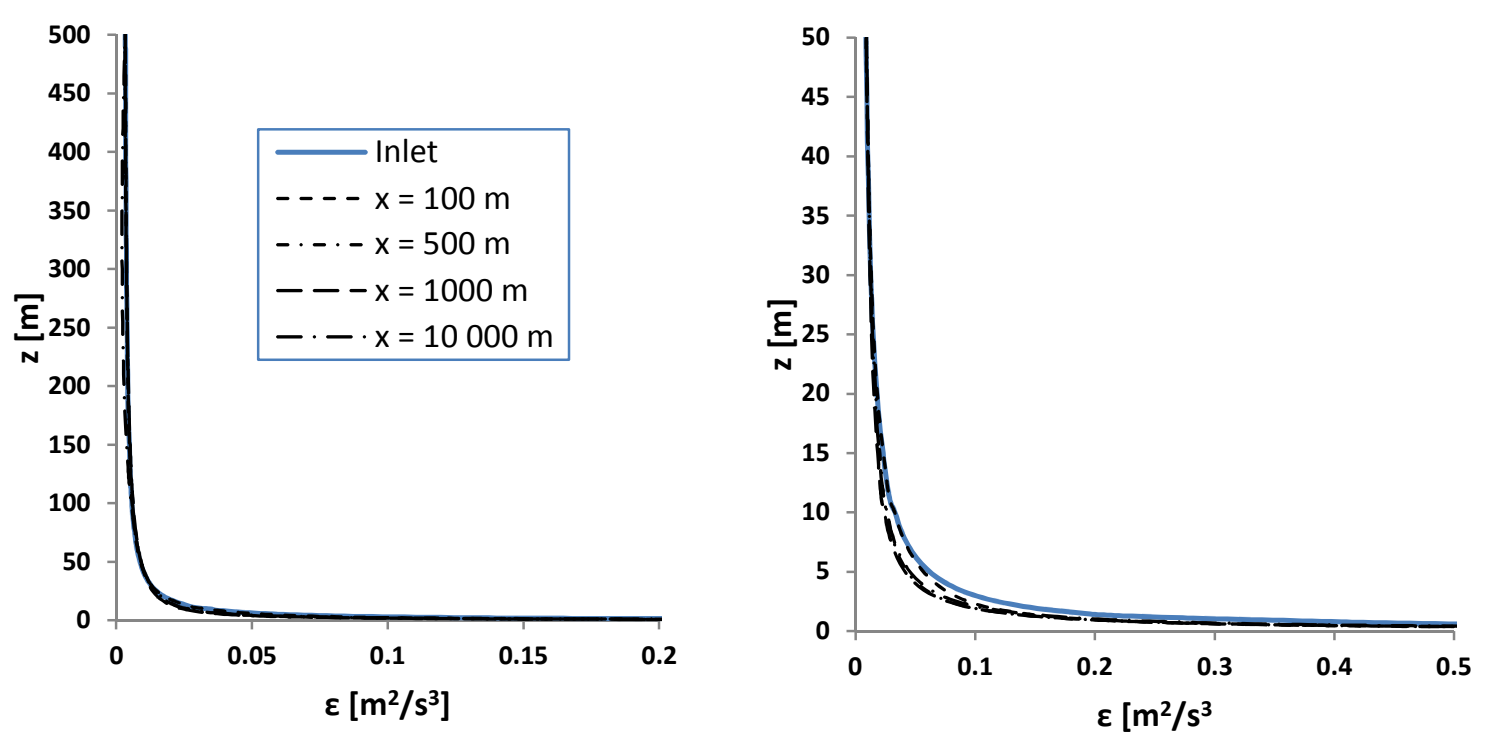

Figure 5-12: Unstable CFD $k-\varepsilon$ model results illustrating streamwise gradients for (a) wind speed $u$; (b) turbulent kinetic energy $k$; and (c) turbulent dissipation rate $\varepsilon$. The right hand column shows the lowest $50 \mathrm{~m}$ of the domain. 


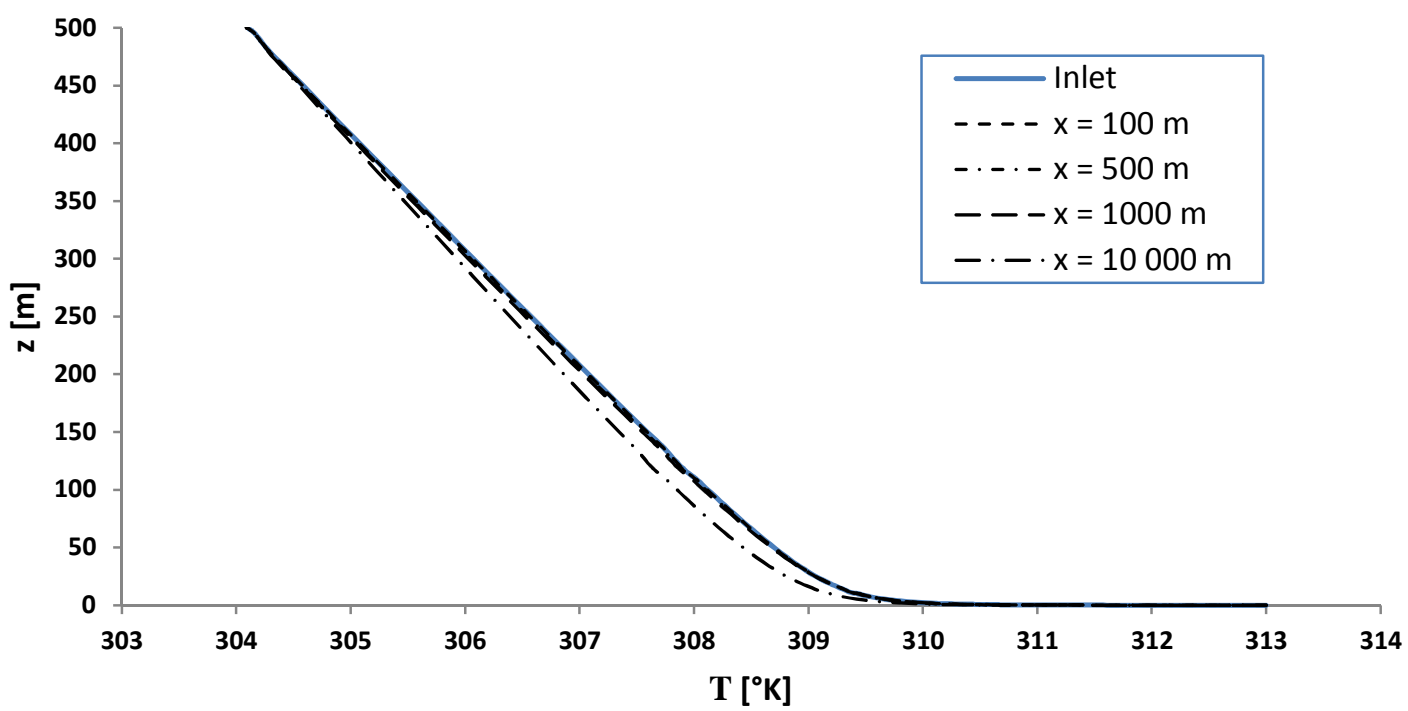

(a)

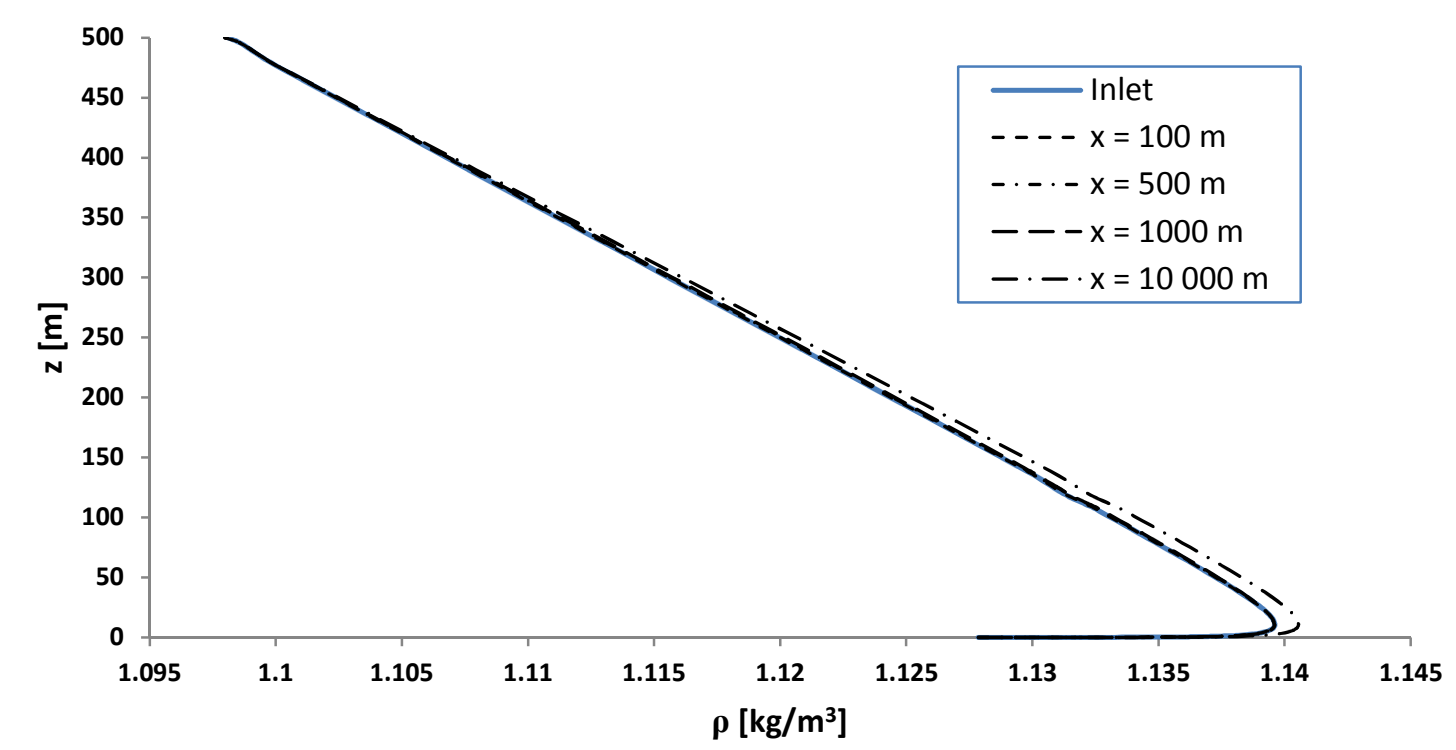

(b)

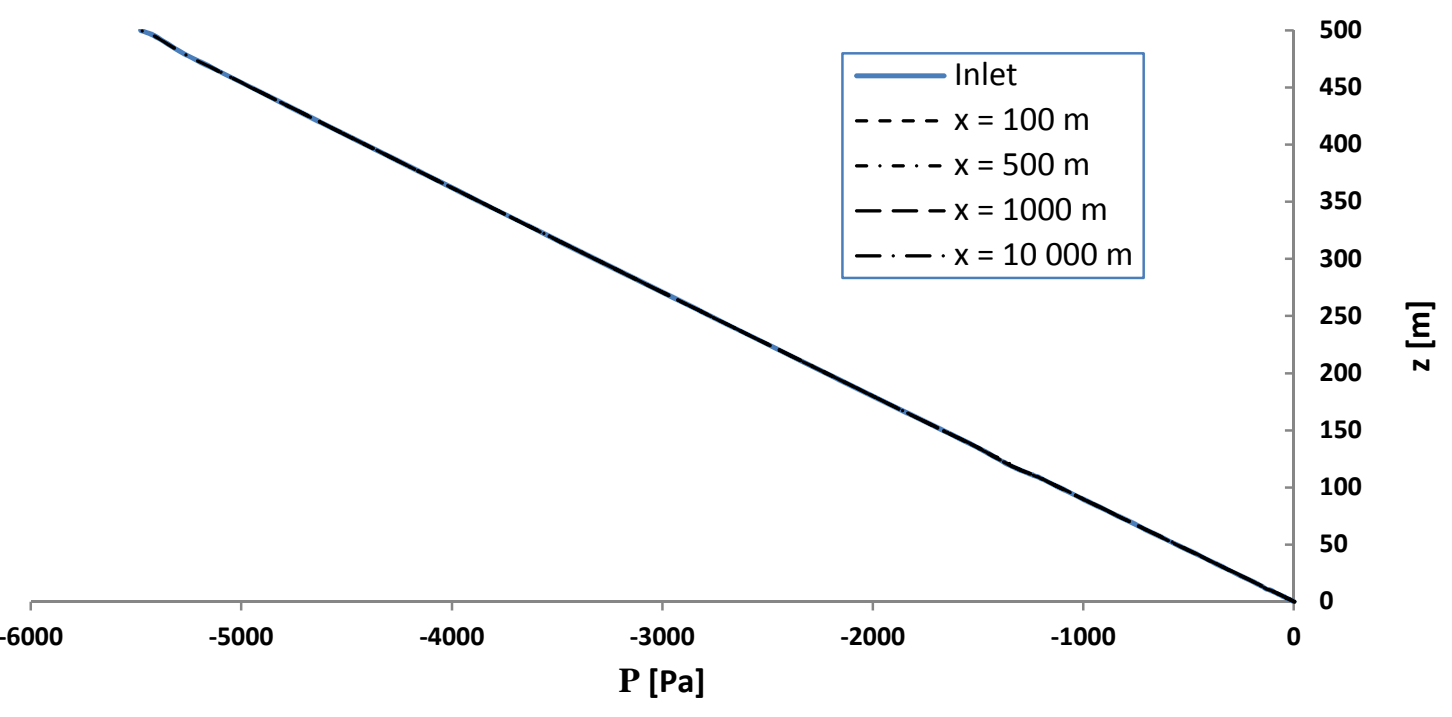

Figure 5-13: Unstable CFD $k-\varepsilon$ model results illustrating streamwise gradients for (a) temperature $T$; (b) density $\rho$; and (c) pressure $P$. 


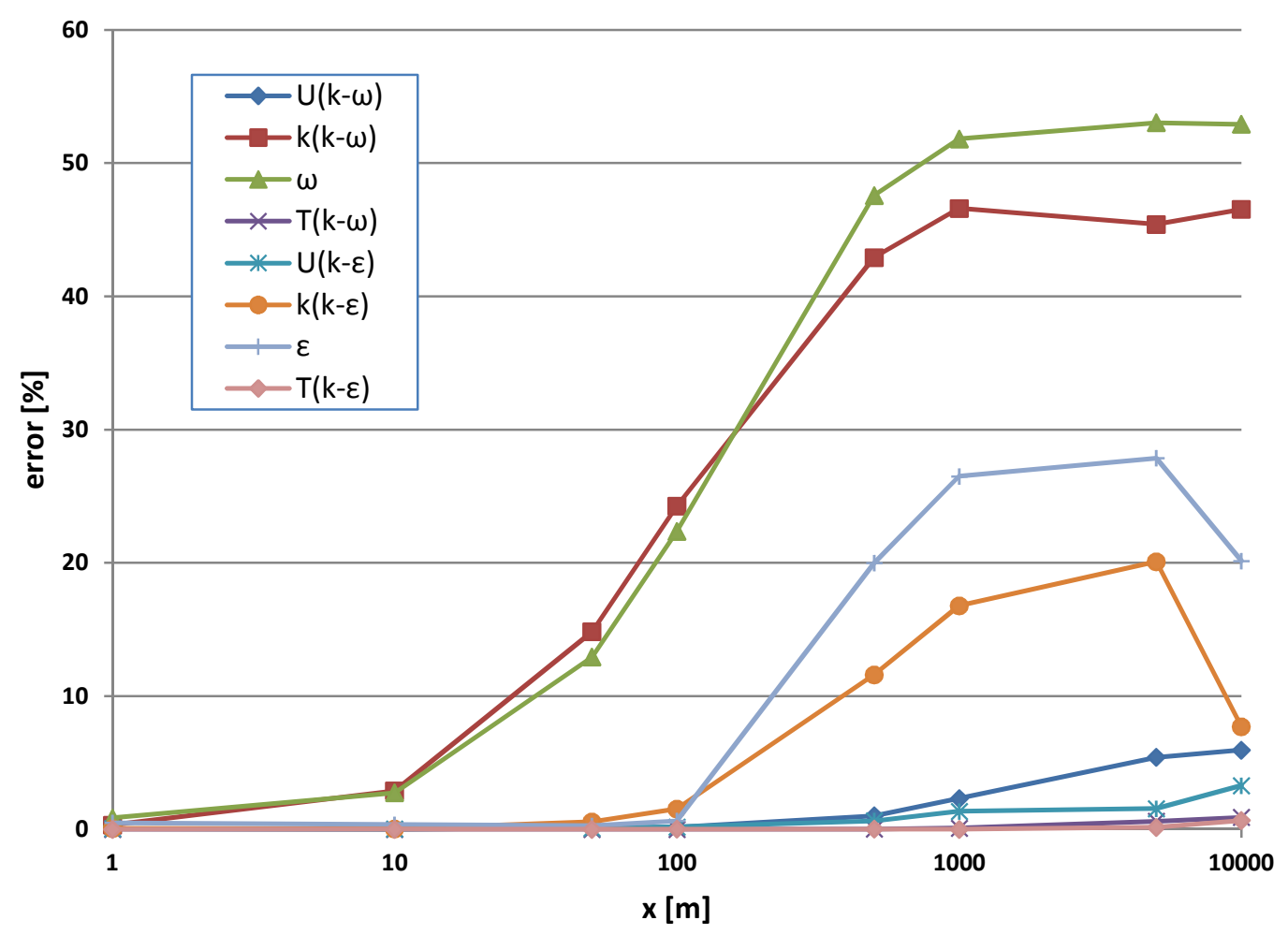

Figure 5-14: Relative change of the flow parameters for the $k-\varepsilon$ and $k-\omega$ turbulence models relative to the inlet for unstable conditions.

The maintenance of the velocity and temperature profiles for all the thermal stratifications were found to be particularly good, with less than $10 \%$ error at a height of $10 \mathrm{~m}$, the point in the domain where the some of the largest inhomogeneity was observed. Other studies have found that the temperature profiles cannot be maintained without inducing numerical instability (Huser et al. 1997, Pontiggia et al. 2009, Meissner et al. 2009), something which has been attained in this study.

The inconsistencies in the $k$-profiles and the value of the turbulent kinetic energy calculated at the first grid point above the wall points to the wall function being inconsistent with the turbulence profiles. This inconsistency is likely to be the reason for the streamwise gradients induced in the flow domain. This is a consistent problem in the CFD simulation of the ABL and has been encountered by many researchers (Blocken et al. 2007a, Hargreaves and Wright 2007, Yang et al. 2008, Franke et al. 2011, Parente et al. 2011b).

Remedial measures have been suggested which includes the reformulation of the wall function from a $k_{s}$ based treatment to a $z_{0}$ based treatment (Blocken et al. 2007b, Parente et al. 2011a). The manipulation of the code in commercial CFD packages like FLUENT or STAR-CCM+ to make these kinds of modifications is not straightforward and is much more easily implemented in open-source software like OpenFOAM (Balogh et al. 2012). The reason is that commercial codes do not 
allow access to the source code, and for this reason the current wall functions provided for use in the code have to suffice. It is suggested though that vendors of commercial codes make wall functions for application to the ABL available, as long as wind engineers make use of CFD, or risk losing these users to open-source options.

The effect of the homogeneity error can be minimised by keeping the domain as short as possible, as it was seen that the errors were small and the profiles very well maintained for the first $500 \mathrm{~m}$ of the domain. It should further be noted that profiles with higher surface roughness, not presented here, proved more difficult to maintain, as the resolution close to the ground was limited by the roughness height and the turbulent kinetic energy produced was also higher. The higher turbulent kinetic energy leads to increased momentum exchange between the fluid layers which lead to an increase in the acceleration of the velocity close to the ground (Blocken et al. 2007b).

Furthermore, the reduction of the wind speed leads to a reduction in the shear produced turbulence, and leads to a more dominant role of the buoyancy produced turbulence. The buoyancy effects therefore start to become significant at lower speeds and increase the observed homogeneity error for profiles of lower wind speed, particularly under unstable conditions, with the solution also taking much longer to converge.

It can be noted that the assumption in surface layer modelling is constant fluxes of momentum and heat with height, equal to the surface shear stress and heat flux. It would be expected that the accuracy of the CFD model will improve if the shear stress and heat flux could be specified at the top of the domain, thus forcing a constant flux of heat and momentum through the boundary layer that is consistent with the theory and the inlet profiles. This sort of boundary treatment in STAR$\mathrm{CCM}+$ unfortunately is also not straightforward, but it is a recommended treatment for future investigations where boundaries can easily be customised, such as in OpenFOAM.

In general then, it is advisable that for every CFD simulation involving ABL flow, the effects of horizontal inhomogeneity first be assessed by performing a simulation in an empty computational domain. This is especially true if the ABL is modelled under the effects of thermal stratification, as for certain combinations of flow variables and surface parameters the error induced in the streamwise direction can be large. It can be concluded that while the $k-\varepsilon$ turbulence model proved to be better equipped to maintain the flow properties along the fetch of the domain, it is known to be less accurate in separated regions, an area where the SST $k$ - $\omega$ turbulence model is known to perform well. It only needs to be shown, thus, that the SST $k-\omega$ turbulence model can adequately maintain the flow properties of the thermally stratified ABL, which has been demonstrated here. A further challenge for future study could be to adapt the SST $k-\omega$ turbulence model constants and boundary conditions to more accurately reflect the Monin-Obukhov similarity theory, as has been done with the $k-\varepsilon$ turbulence model. 


\section{CFD SIMULATION OF FLOW OVER A COSINE HILL}

Having set up a CFD method for simulating the real atmosphere under the influence of gravity in the previous chapter, we now turn our attention to how well such a model can predict separated flow situations. The CFD models, modified to account for gravity effects, therefore need to be validated against experimental data. These measurements were obtained in the thermally stratified wind tunnel of the University of Tokyo, using three-dimensional laser Doppler anemometry (Takahashi et al. 2005). The model is an axisymmetric cosine shaped hill of scale 1/1000, with the height $h$ defined in terms of the radius $r$ by:

$$
h(r)=\left\{\begin{array}{lr}
H\left[1+\cos \left(\frac{2 \pi r}{R_{b}}\right)\right], & \text { if } r<R_{b} \\
0, & \text { otherwise }
\end{array}\right.
$$

where $R_{b}$ is the radius of the hill base and has a value of $0.42 \mathrm{~m}$, with the maximum height of the hill $H$ given as $0.2 \mathrm{~m}$.

The boundary conditions for the velocity and turbulent kinetic energy were derived for the results presented by Takahashi et al. (2005) for the incident flow in the wind tunnel, and the results of the flow field around the hill model for the mean streamwise velocity and the turbulent kinetic energy were used for comparison to the CFD results.

The turbulence models tested included the $k-\varepsilon$ turbulence model and the SST $k-\omega$ turbulence model modified for gravity effects as described in the Chapter 5. In addition two other steady RANS models were used namely: the realisable $k-\varepsilon$ turbulence model and the Spalart-Allmaras turbulence model. An implicit unsteady detached eddy simulation (DES) model, derived from the SST $k-\omega$ turbulence model, was also employed.

The conditions for the neutral or unheated wind tunnel were used. The parameters derived from the incident (model position in wind tunnel without the model) profile of velocity are shown in Table 6-1. The reference velocity $\left(u_{H}\right)$ used was the velocity at the height of the model in the empty wind tunnel, and the reference height was the model height $(H)$. The height of the boundary layer $(\delta)$ was found to be about $0.7 \mathrm{~m}$ with a velocity here of $1.42 \mathrm{~m} / \mathrm{s}$, which was the maximum velocity the profile attained. The mean flow velocity $\left(u_{m}\right)$ at the model position in the wind tunnel without the model was found to be $1.0572 \mathrm{~m} / \mathrm{s}$. Additionally the turbulent intensities of the incident velocity profile were provided as a root mean square value of the velocity fluctuations in the $x$-, $y$ - and $z$-directions. The values of turbulent kinetic energy could therefore be calculated by:

$$
k=\frac{1}{2}\left(\overline{u^{\prime 2}}+\overline{v^{\prime 2}}+\overline{w^{\prime 2}}\right)
$$


Table 6-1: Parameters derived from neutral wind tunnel incident flow

\begin{tabular}{cccccccccc}
\hline $\boldsymbol{z}_{\mathbf{0}}$ & $\boldsymbol{u}_{\boldsymbol{H}}$ & $\boldsymbol{H}$ & $\boldsymbol{\delta}$ & $\boldsymbol{T}_{\mathbf{0}}$ & $\boldsymbol{u}_{\boldsymbol{\delta}}$ & $\mathbf{L}$ & $\boldsymbol{u}_{*}$ & $\boldsymbol{\kappa}$ & $\boldsymbol{k}_{\boldsymbol{s}}$ \\
{$[\mathrm{m}]$} & {$[\mathrm{m} / \mathrm{s}]$} & {$[\mathrm{m}]$} & {$[\mathrm{m}]$} & {$\left[{ }^{\circ} \mathrm{C}\right]$} & {$[\mathrm{m} / \mathrm{s}]$} & {$[\mathrm{m}]$} & {$[\mathrm{m} / \mathrm{s}]$} & $/$ & {$[\mathrm{m}]$} \\
\hline 0.000676 & 1.174 & 0.2 & 0.7 & 17 & 1.42 & $\infty$ & 0.0845 & 0.41 & 0.00507 \\
\hline
\end{tabular}

\subsection{Computational Domain and Grid Generation}

The working section of the wind tunnel is $2.2 \mathrm{~m}$ wide and $1.8 \mathrm{~m}$ high. As the profiles for the incident flow on the hill were available it was decided to place the inlet of the domain at least $8 H$ upstream of the hill (Franke et al. 2011) and therefore the model was placed $2 \mathrm{~m}$ downstream from the inlet. It is further recommended that when using an outflow boundary it must be placed at least $15 \mathrm{H}$ from the obstruction (Franke et al. 2011), a downstream length of $6 \mathrm{~m}$ was chosen to resemble the overall size of the wind tunnel and to allow for flow recovery. To save on computational requirements only half of the model and wind tunnel was simulated, with the addition of a symmetry boundary along the central lengthwise axis of the domain.

A three-dimensional STL (stereo lithography) surface file was created with dimensions given above, using CAD software. This surface file could then be imported into STAR-CCM+ as a new fluid region and meshed as a threedimensional fluid domain. The region was split into seven boundaries corresponding to the top and side walls, the symmetry plane, the inlet and the outlet of the domain, as well as the wind tunnel floor and the hill model itself.

The surface was meshed using the "trimmer" meshing model, the "surface remesher" and the "prism layer mesher". The "prism layers" were only applied to the bottom wall or floor of the wind tunnel and the hill surface, by disabling this meshing model on the top (ceiling) and side walls. The reference values used in the meshing models are listed in Table 6-2. The mesh around the hill was refined using the "trimmer wake refinement" option. Four sets of "wake" refinements were specified, upstream, downstream, vertically and laterally from the hill model, with the inputs given in Table 6-3.

The resulting three-dimensional mesh had 602796 hexahedral cells with a $z_{p}$ of $0.0053 \mathrm{~m}$ over the wind tunnel floor and over the hill surface (so that $z_{p}>k_{s}$ ), and is shown in Figure 6-1. A grid sensitivity analysis was carried out to determine the solution error associated with the discretisation, using the procedure of Celik et al. (2008), which is presented in Appendix D. The mesh thus specified proved to provide acceptable discretization error, based on the reattachment length, velocity and turbulent kinetic energy in the wake of the hill. The resulting mesh was used for all the subsequent CFD calculations in this section, using the different turbulence models. 
Table 6-2: Inputs for meshing model

\begin{tabular}{llc}
\hline Reference Node Name & Property Name & Value \\
\hline Base Size & Value & $40 \mathrm{~mm}$ \\
CAD Projection & Project to CAD & True (default) \\
Maximum Cell Size>Relative Size & Percentage of Base & $100 \%$ \\
Number of Prism Layers & Number of Prism Layers & 3 \\
Prism Layer Stretching & Prism Layer Stretching & 1.05 \\
Prism Layer Thickness $>$ Absolute & Absolute Size & $33.3 \mathrm{~mm}$ \\
Surface Curvature & \# Pts/circle & 36 (default) \\
Surface Growth Rate & Surface Growth Rate & 1.3 (default) \\
Surface Proximity & \# Points in a gap & 2.0 (default) \\
Surface Proximity & Search Floor & 0.0 (default) \\
Surface Size $>$ Relative Minimum Size & Percentage of Base & 25 \\
Surface Size $>$ Relative Target Size & Percentage of Base & $100 \%$ \\
Template Growth Rate & Default Growth Rate & Fast (default) \\
Template Growth Rate & Boundary Growth Rate & None (default) \\
\hline
\end{tabular}

Table 6-3: Inputs for mesh refinement

\begin{tabular}{|c|c|}
\hline Property Name & Value \\
\hline Relative/Absolute> Relative to base & $25 \%$ \\
\hline Boundary Growth Rate & None (default) \\
\hline Boundaries/Feature Curves & "Hill Model" \\
\hline Default Growth Rate & Fast (default) \\
\hline \multicolumn{2}{|c|}{ Wake Refinement Downstream } \\
\hline Distance & $3.0 \mathrm{~m}$ \\
\hline Direction & {$[1.0,0.0,0.0]$} \\
\hline \multicolumn{2}{|c|}{ Wake Refinement Upstream } \\
\hline Distance & $0.75 \mathrm{~m}$ \\
\hline Direction & {$[-1.0,0.0,0.0]$} \\
\hline \multicolumn{2}{|c|}{ Wake Refinement Vertically } \\
\hline Distance & $0.2 \mathrm{~m}$ \\
\hline Direction & {$[0.0,0.0,1.0]$} \\
\hline \multicolumn{2}{|c|}{ Wake Refinement Laterally } \\
\hline Distance & $0.2 \mathrm{~m}$ \\
\hline Direction & {$[0.0,-1.0,0.0]$} \\
\hline
\end{tabular}



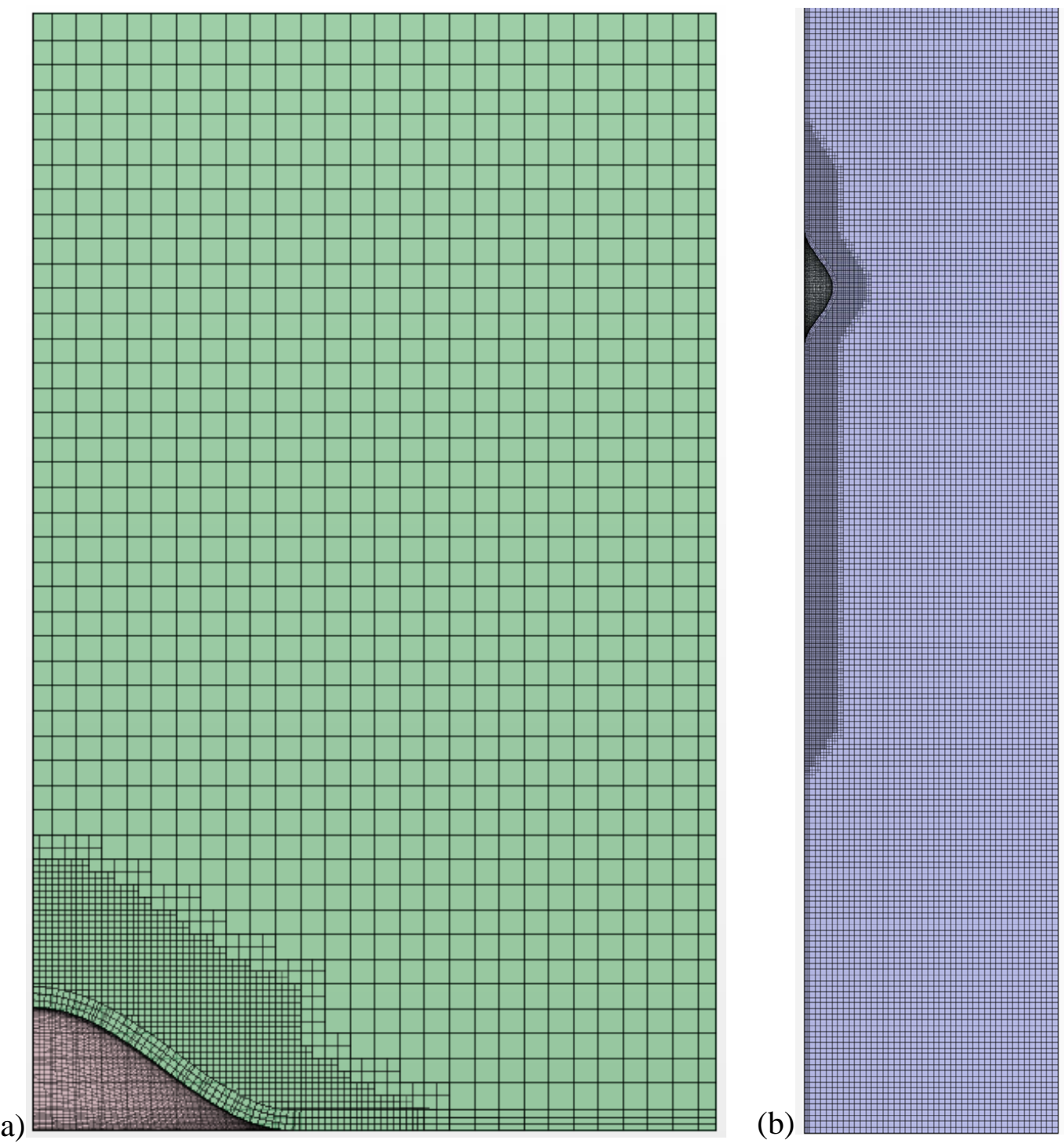

Figure 6-1: Computational grid: (a) section through centre of hill looking down $x$ direction in $z-y$ plane; (b) looking at symmetry plane down $y$-direction in $x-z$ plane.

\subsection{Flow Physics Solution Specification}

The wind tunnel simulation boundary conditions and physics models were chosen to reflect as closely as possible the simulations carried out in Chapter 5. Thus, the possible effect of the source terms, added to account for gravity, on the performance of the turbulence models under adverse pressure gradients could be assessed. 


\subsubsection{Physics models and solvers}

The physics models used to specify the steady RANS simulations were as follows: the fluid space was specified as three-dimensional and the time specification was steady. The material specified was air with the same properties as given in Chapter 5. The density was calculated through the ideal gas law. The segregated flow solver was chosen, which achieves pressure-velocity coupling through the SIMPLE algorithm, and solves the total energy equation through the segregated fluid temperature model, which uses temperature as the independent variable (CD-adapco Inc. 2011). Convection of momentum and energy was solved using the second-order upwind scheme. The turbulent viscous regime was chosen and the Reynolds-Averaged Navier-Stokes (RANS) turbulence models were specified.

A reference pressure of $101325 \mathrm{~Pa}$ was used, which is applied by default at the point $[0,0,0]$ in the fluid domain (CD-adapco Inc. 2011). The flow was initialised using the inlet boundary conditions for velocity, temperature, and turbulence quantities defined below, as well as zero pressure. The solver employed the Gauss-Seidel relaxation scheme with under-relaxation factors given in Table 6-4. The solution was run until the normalised residuals of continuity, momentum, energy and turbulence had converged.

Table 6-4:Under-relaxation factors for flow simulations

\begin{tabular}{ll}
\hline Pressure & 0.3 \\
Velocity & 0.7 \\
Energy & 0.9 \\
$k-\varepsilon$ Turbulence & 0.8 \\
$k-\omega$ Turbulence & 0.8 \\
Spalart-Allmaras Turbulence & 0.7 \\
Turbulent Viscosity & 1.0 \\
\hline
\end{tabular}

The implicit unsteady solver was used for the DES turbulence model, and the initial conditions were the converged flow solutions achieved using the steady state SST $k-\omega$ turbulence model. Further the same physics models were used as for the steady RANS simulations. A time step of $0.005 \mathrm{~s}$ was specified for a maximum physical time of $5 \mathrm{~s}$. The maximum inner iterations was set to 15 iterations per time step as this was found to be sufficient for the normalised residuals to decrease by at least three orders of magnitude. For a cell length of $0.01 \mathrm{~m}$ and a velocity of $1.5 \mathrm{~m} / \mathrm{s}$, the time step gave a Courant-Friedrichs-Lewy number of 0.75 . In addition a "solution history" file was created for turbulent kinetic energy and velocity which stored the solution data for every $0.01 \mathrm{~s}$. 
Furthermore, all the models had the momentum and energy source terms, defined in Chapter 5, specified. The $k-\varepsilon$ and $k-\omega$ turbulence models further had the turbulence sources due to gravity, as defined in Chapter 5, specified. The SpalartAllmaras turbulence model is a one-equation RANS model and therefore the turbulent production due to buoyancy cannot be specified as it can for the two equation models. Under the neutral wind tunnel conditions there should, however, not be any buoyant turbulence production. The turbulence model constants for each turbulence model used are shown in Table 6-5.

Table 6-5: Turbulence model constants

\begin{tabular}{lcccccccc}
\hline \multirow{2}{*}{ Standard $\boldsymbol{k}-\boldsymbol{\varepsilon}:$} & $C_{\varepsilon 1}$ & $C_{\varepsilon 2}$ & $C_{\mu}$ & $\sigma_{k}$ & $\sigma_{\varepsilon}$ & & & \\
& 1.1523 & 1.92 & 0.0333 & 1.0 & 1.3 & & & \\
\hline \multirow{2}{*}{ Realisable $\boldsymbol{k}-\boldsymbol{\varepsilon}:$} & $C_{\varepsilon 1}$ & $C_{\varepsilon 2}$ & $C_{\mu}$ & $\sigma_{k}$ & $\sigma_{\varepsilon}$ & & & \\
& 1.1523 & 1.90 & 0.0333 & 1.0 & 1.2 & & & \\
\hline \multirow{2}{*}{ SST $\boldsymbol{k}$ - $\boldsymbol{\omega}:$} & $\alpha_{1}$ & $\beta_{1}$ & $\beta_{*}$ & $\sigma_{k 1}$ & $\sigma_{\omega 1}$ & $\beta_{2}$ & $\sigma_{k 2}$ & $\sigma_{\omega 2}$ \\
& 0.31 & 0.075 & 0.09 & 0.85 & 0.5 & 0.0828 & 1.0 & 0.856 \\
\hline \multirow{2}{*}{ Spalart-Allmaras: } & $C_{b 1}$ & $C_{b 2}$ & $C_{p r o d}$ & $C_{v 1}$ & $C_{w 2}$ & $C_{w 3}$ & $\kappa$ & $\sigma_{\mathrm{v}}$ \\
& 0.1355 & 0.622 & 2.0 & 7.1 & 0.3 & 2.0 & 0.41 & $2 / 3$ \\
\hline \multirow{2}{*}{ SST $\boldsymbol{k}$ - $\boldsymbol{\omega}$ DES: } & $C_{D E S k \omega}$ & $C_{D E S k \varepsilon}$ & $C_{D E S t}$ & & & & & \\
& 0.78 & 0.61 & 1.0 & & & & & \\
\hline
\end{tabular}

\subsubsection{Boundary conditions}

As the CFD had to contend with separated flow, it was considered important to get as high a possible grid resolution close to the wind tunnel floor and the hill. Therefore the mesh was generated so that $z_{p}$ was just greater than the value specified for the roughness height $k_{s}$. This yielded a $z^{+}$value of between 10 and 50 over the floor and the hill. This situation therefore required the use of a blended wall function and can be specified in STAR-CCM+ by the all $z^{+}$wall treatment, which is a hybrid approach between the low- $Z^{+}$and high- $z^{+}$wall functions (CD-adapco Inc. 2011). In the case of the $k-\varepsilon$ turbulence models the Wolfstein two-layer formulation was used. The value of the roughness height was specified on the floor of the wind tunnel, which was modelled as a rough wall with the no-slip shear stress specification, as well as the hill which received the same boundary treatment. Additionally the floor and hill had the temperature $T_{0}$ from Table 6-1 specified.

The side wall and ceiling were selected as smooth walls with adiabatic thermal specification and with the no-slip condition also applied. The symmetry boundary was used on the symmetry plane of the wind tunnel and model, while and the downstream flow outlet was specified as a "flow-split outlet" with a split ratio of one. 
The upstream inlet of the domain was specified as a "velocity inlet". For all the simulations the adiabatic lapse rate temperature was specified using a user "field function" relative to the floor temperature, although the change in temperature with height was virtually negligible. The $x$-direction velocity in each case was specified through a user "field function" by Equation 2-24 (with zero $u_{y}$ and $u_{z}$ ), using the values given in Table 6-1. The $k-\varepsilon$ and $k-\omega$ turbulence models had the turbulent kinetic energy that was measured in the incident flow specified at the inlet. This was achieved by reading the wind tunnel data into STAR-CCM+ using a table and then creating a "field function" that accesses the table data and interpolates it for each mesh centroid at the inlet, using splines. The SpalartAllmaras turbulence model required the definition of turbulence intensity $I$ and turbulent length scale $l$ through:

$$
\begin{gathered}
I=\frac{\left(\frac{2}{3} k\right)^{1 / 2}}{u_{m}} \\
l=\frac{\kappa Z}{1+\kappa z / l_{\infty}}
\end{gathered}
$$

where $u_{m}$ is the mean flow velocity and $l_{\infty}$ is the asymptotic mixing length (Griffiths and Middleton 2010) given by:

$$
l_{\infty}=\kappa \delta
$$

Here $\delta$ is the boundary layer height given in Table 6-1. The inlet values for $\varepsilon$ and $\omega$ were specified by defining "field functions" for Equation 3-13 and Equation 3-19 respectively.

\subsection{Comparison of Measurements with CFD Results}

The CFD and experimental results could be compared using hit rates as a quality assessment metric. This quantity indicates the fraction of the $N$ measurement locations where the CFD results are within a $25 \%$ interval of the experimental data (Britter and Schatzmann 2007). Normalised values are used, with the reference wind speed and hill height used for normalisation.

The distributions of $x$-velocity and turbulent kinetic energy along the central plane of the hill (on the symmetry plane) were normalised with the reference velocity $u_{H}$ and compared with vertical distributions of the wind tunnel data at values of $x / H$ of $0,1.125,2.25,3,4,5.5$ and 7 , where 0 corresponds to the centre point of the hill, and the other points are all in the wake. In all cases the measured data corresponds to the published data of Takahashi et al. (2005). 


\subsection{Results and Discussion}

The results of the comparison of the different turbulence models for flow over the cosine shaped hill against measured data are shown in Figure 6-2 for the streamwise velocity and in Figure 6-3 for the turbulent kinetic energy. The results for the DES are the time averaged results over the $5 \mathrm{~s}$ of the simulation.

The velocity results reveal that all the turbulence models correspond well with the measured data of the velocity distributions at the top of the hill, and then again after about four hill heights downstream. It is in the recirculating flow zone and immediately after it where the turbulence models tended to give different results. It was found that the realisable $k-\varepsilon$ turbulence model predicted no separation in the wake of the hill, with the $x$-direction velocity never becoming negative. The standard $k-\varepsilon$ turbulence model yielded some separation but still under predicted the size and extent of the separation bubble, suggesting faster flow recovery. On the other hand the Spalart-Allmaras turbulence model over predicted the influence of the adverse pressure gradient with larger separation bubble prediction than that shown by the measured data. It was the SST $k$ - $\omega$ turbulence model that gave the best prediction of the separated region directly in the wake of the hill, and also showed the best correspondence with the measured data for the size of the separation bubble.

The time averaged DES data showed a similar trend as the steady state SST $k-\omega$ turbulence model, but tended to over predict the separated zone downstream of the hill compared to the steady state model. There may be several reasons for this, which include that perhaps the $5 \mathrm{~s}$ that was simulated was not sufficient to capture the full range of transient behaviour in the wake of the hill. It should be noted that the sampling time for one wind tunnel measurement was $45 \mathrm{~s}$ with 2000 samples for one measurement. It is therefore suggested that the DES be simulated for a longer time span. It was, however, not feasible for this particular study as the computational time required for the DES simulation was considerably more than for the steady state RANS turbulence models. It should also be noted that there were considerably more transient effects in the initial few seconds of the simulation as the initial conditions were overcome. It is therefore suggested that the first few seconds should not be included in the time average as this part of the time simulation is still under the influence of the initial conditions.

It was nevertheless found that the steady state RANS models were capable of fairly accurate prediction of the separated region, particularly the SST $k-\omega$ turbulence model, which is consistent with the findings of Yang et al. (2008). It is furthermore achieved with considerably less computational requirements than for unsteady simulations, making high turnover of different simulations possible. Unless one was interested in transient effects like vortex shedding, which was observed in the unsteady DES simulation, it is suggested that steady state turbulence models can suffice. 


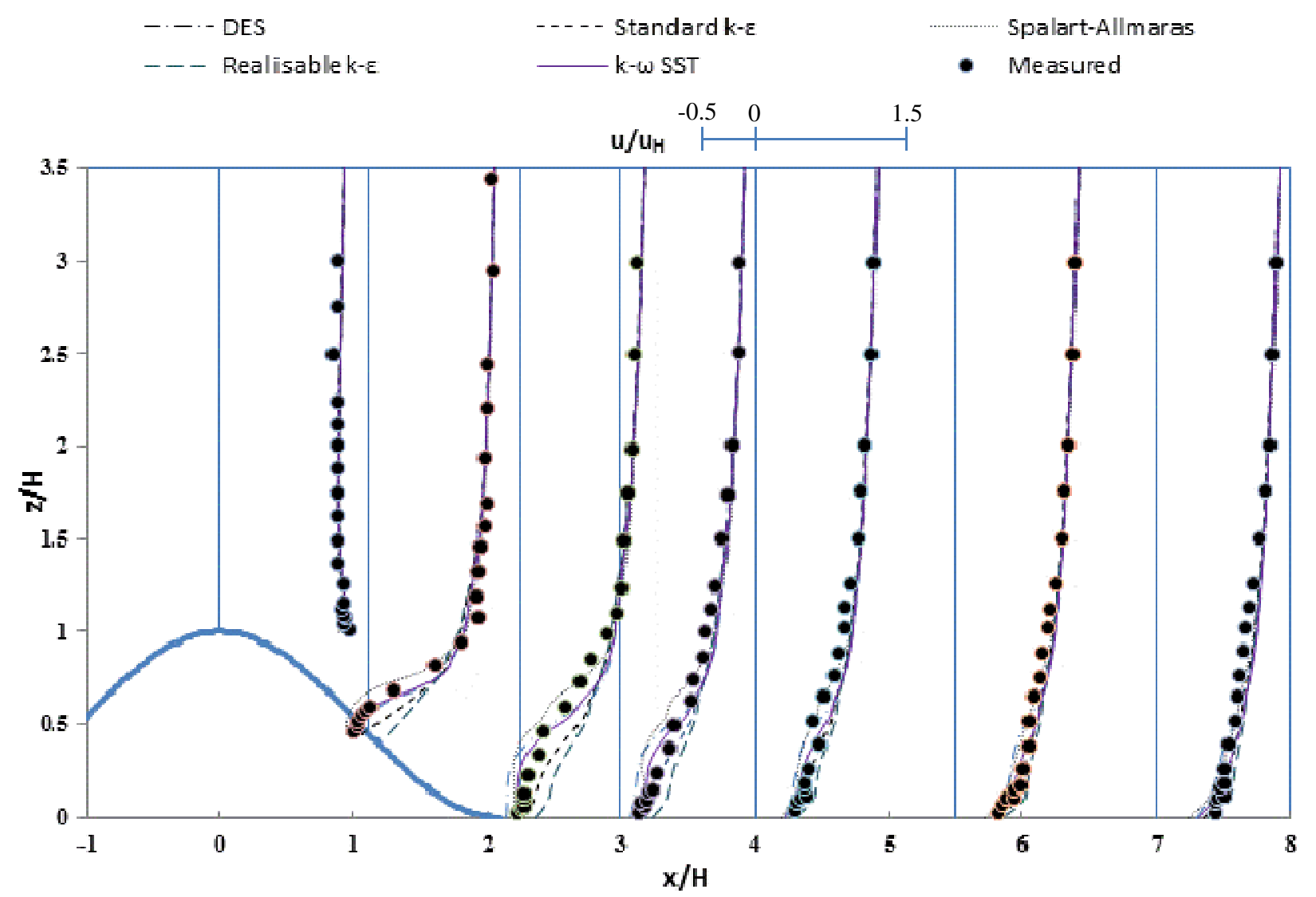

Figure 6-2: Comparison of the horizontal velocity results against measurement
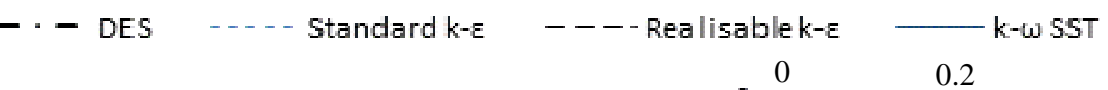

- Measured
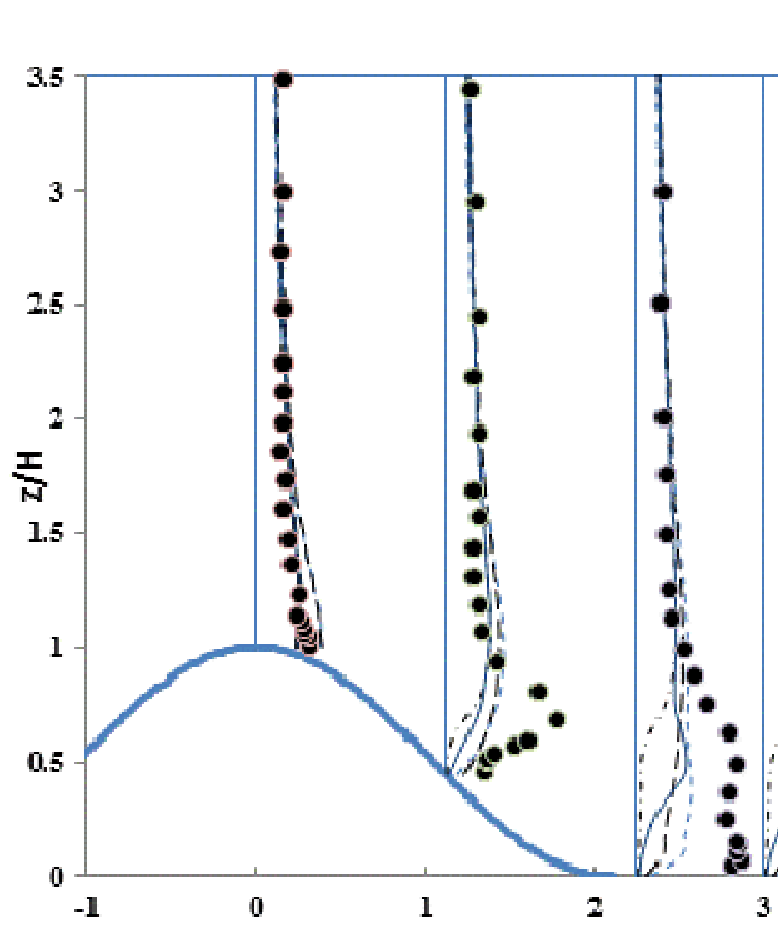

$\mathrm{k} / \mathrm{u}_{\mathrm{H}}^{2}$
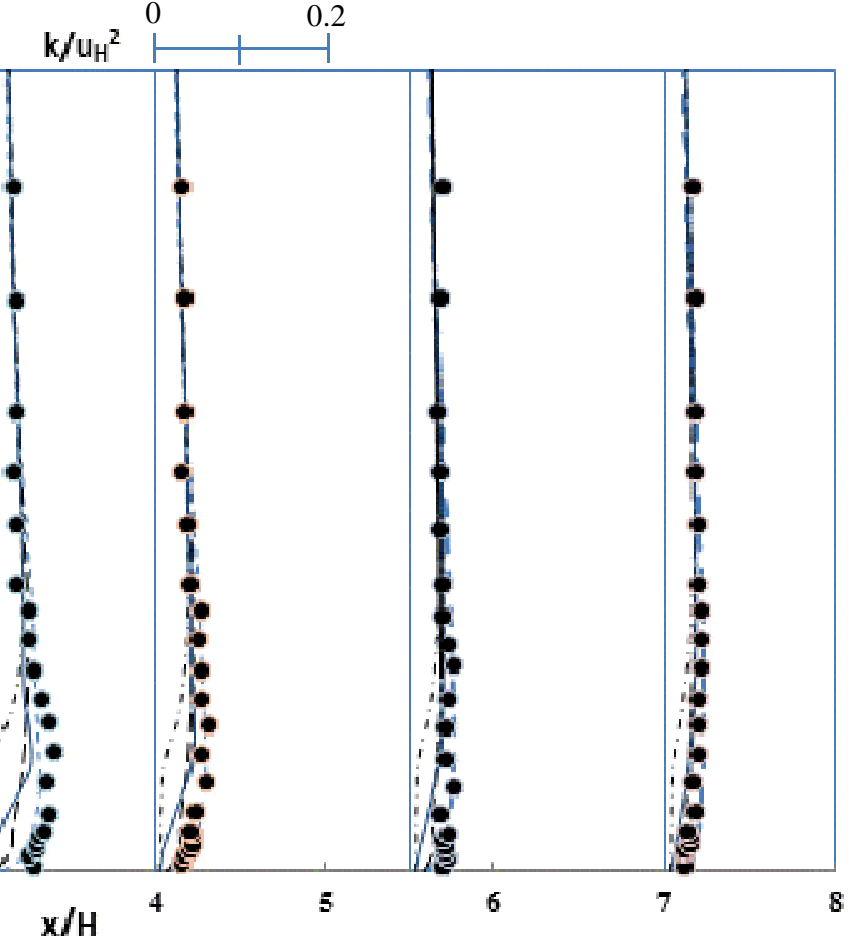

Figure 6-3: Comparison of the turbulent kinetic energy results against measurement 
The results of the hit rates analysis are shown in Table 6-6. It is seen that the percentage scores of the SST $k-\omega$ turbulence model and the standard $k-\varepsilon$ turbulence model were the highest for velocity, suggesting that they most closely corresponded to the measurements. The SST $k$ - $\omega$ turbulence model was better at predicting the flow in the immediate wake of the hill while the standard $k-\varepsilon$ turbulence model was better at predicting the flow recovery further downstream. It can therefore be recommended that both of these models should be used in subsequent investigations.

Table 6-6: Hit rate values for simulations over cosine hill

\begin{tabular}{lcc}
\hline Cases & Streamwise Velocity [\%] & Turbulent Kinetic Energy [\%] \\
\hline Spalart-Allmaras & 69.1 & $/$ \\
$k-\omega$ SST & 71.9 & 36.9 \\
Realisable $k-\varepsilon$ & 67.6 & 44.1 \\
Standard $k-\varepsilon$ & 71.9 & 31.5 \\
DES & 69.1 & 30.6 \\
\hline
\end{tabular}

It was the turbulent kinetic energy distributions that the turbulence models had the hardest time reproducing. This was found to be particularly true in the immediate wake regions of the flow, where the measurements showed a much more pronounced peak close to the ground than predicted by any of the turbulence models. The realisable $k-\varepsilon$ turbulence model provided the best prediction of the turbulent kinetic energy distributions, but this is attributed to the downstream profiles. These results are similar to those found by Balogh et al. (2012) using FLUENT and OpenFOAM. They found that the results could be marginally improved, particularly for the prediction of the turbulent kinetic energy distributions, by modifying the wall treatments to be consistent with the velocity and turbulence inlet profiles in OpenFOAM (Balogh et al. 2012). This kind of wall treatment, however, is not available to users of STAR-CCM+, and its implementation is made difficult as access to the source-code is unavailable.

The flow measurements were also carried out for stable and unstable boundary layers in the thermally stratified wind tunnel. In was, however, found that the temperature profiles enforced on the incident flow could not be maintained by the CFD code. The temperature gradients were found to be too large for the model to handle and considerable heat conduction occurred resulting in a severe distortion of the inlet profiles. It should be noted that the CFD equations were modified to account for the temperature and velocity gradients typically occurring in the atmosphere and not the severe gradients imposed by the wind tunnel. It is suggested that perhaps the wind tunnel results for the thermally stratified boundary layer can be compared with a full scale CFD simulation of the 1/1000 scale model, where the change in temperature with height is more natural. The question therefore arises if scaled down wind tunnel results can be compared to full scale CFD simulations. This will be touched upon in the next chapter. 


\section{CFD SIMULATION OF STRATIFIED ABL FLOW OVER AN IDEALISED TRANSVERSE DUNE}

It was shown in previous chapters that CFD can be used to accurately model the ABL under different thermal stratifications, and that the turbulence models employed perform adequately well for flow separation. It was now possible to investigate the flow field around an idealised two-dimensional transverse dune immersed in a thermally stratified ABL. The standard $k-\varepsilon$ turbulence model and the SST $k$ - $\omega$ turbulence model, modified for gravity effects, were used to model the flow field as they were found to provide the best predictions of flow separation in the previous chapter. The dune geometry was a scaled up version of the dune modelled by Liu et al. (2011), and is described in Table 7-1.

Table 7-1: Dune geometry parameters

\begin{tabular}{lc}
\hline Parameter & Value \\
\hline Dune Height (h) & $10 \mathrm{~m}$ \\
Windward Base Length & $56.71 \mathrm{~m}$ \\
Leeward Base Length & $17.32 \mathrm{~m}$ \\
Windward Slope Angle & $10^{\circ}$ \\
Leeward Slope Angle & $30^{\circ}$ \\
\hline
\end{tabular}

The parameters describing the thermally stratified ABL are given in Table 7.2. The roughness length, von Karman constant and reference velocities were chosen so that similarity existed between the neutral ABL and the boundary layer described by Liu et al. (2011) in their wind tunnel study. The heat fluxes for the unstable and stable atmospheres were chosen to correspond to the values calculated from the interdune in Chapter 4. The surface roughness height was specified as:

$$
k_{s}=z_{0}
$$

This is the absolute minimum value and was chosen to ensure that the grid resolution close to the ground could be as fine as possible. The results of the neutral CFD simulation could be compared to the measurements of Liu et al. (2011) who recorded the flow over a 1/400 scale model of the dune used here in a blow-type non-circulation wind tunnel with a PIV (particle image velocimetry) system.

Table 7-2: Parameters describing the different stratified atmospheres

\begin{tabular}{lccccccccc}
\hline & $\begin{array}{c}\boldsymbol{z}_{\mathbf{0}} \\
{[\mathrm{m}]}\end{array}$ & $\begin{array}{c}\boldsymbol{u}_{\boldsymbol{h}} \\
{[\mathrm{m} / \mathrm{s}]}\end{array}$ & $\begin{array}{c}\boldsymbol{h} \\
{[\mathrm{m}]}\end{array}$ & $\begin{array}{c}\dot{\boldsymbol{q}}_{\mathbf{0}} \\
{\left[\mathrm{W} / \mathrm{m}^{2}\right]}\end{array}$ & $\begin{array}{c}\boldsymbol{T}_{\mathbf{0}} \\
{\left[{ }^{\circ} \mathrm{C}\right]}\end{array}$ & $\begin{array}{c}\mathbf{L} \\
{[\mathrm{m}]}\end{array}$ & $\begin{array}{c}\boldsymbol{u}_{*} \\
{[\mathrm{~m} / \mathrm{s}]}\end{array}$ & $\begin{array}{c}\boldsymbol{\kappa} \\
/\end{array}$ & $\begin{array}{c}\boldsymbol{k}_{\boldsymbol{s}} \\
{[\mathrm{m}]}\end{array}$ \\
\hline Neutral & 0.04 & 6.9 & 10 & 0 & 25 & $\infty$ & 0.499 & 0.40 & 0.04 \\
Stable & 0.04 & 6.9 & 10 & -30 & 10 & 348.0 & 0.487 & 0.40 & 0.04 \\
Unstable & 0.04 & 6.9 & 10 & 400 & 40 & -38.5 & 0.555 & 0.40 & 0.04 \\
\hline
\end{tabular}




\subsection{Computational Domain and Grid Generation}

The minimum upstream distance of the inlet from an obstruction should be at least $8 h$ (Franke et al. 2011). The inlet was therefore placed $150 \mathrm{~m}$ from the crest of the dune. It is further recommended that when using an outflow boundary it must be placed at least $15 \mathrm{~h}$ from the obstruction (Franke et al. 2011), and therefore this boundary was placed $300 \mathrm{~m}$ from the dune crest to ensure sufficient flow recovery. In addition the domain was made $350 \mathrm{~m}$ high to ensure minimal blockage, and $100 \mathrm{~m}$ wide to provide enough space for a sufficient number of cells to be added in the central part of the flow domain, which was needed for grid refinement. Once again a three-dimensional STL surface file was generated using CAD software. The surface was imported into STAR-CCM+ as a new fluid region and meshed as a three-dimensional fluid domain. The region was split into seven boundaries corresponding to the top of the domain, the two sides, the upstream inlet and the downstream outlet of the domain, as well as the ground and the dune.

The surface was meshed using the "trimmer" meshing model, the "surface remesher" and the "prism layer mesher". The "prism layer" was applied to ground and the dune surface. The reference values used in the meshing models are listed in Table 7-3. The mesh around the dune was refined using the "trimmer wake refinement" option. Three sets of "wake" refinements were specified, upstream, downstream, and vertically from the dune surface, with the inputs given in Table 7-4.

Table 7-3: Inputs for meshing model

\begin{tabular}{llc}
\hline Reference Node Name & Property Name & Value \\
\hline Base Size & Value & $5 \mathrm{~m}$ \\
CAD Projection & Project to CAD & True (default) \\
Maximum Cell Size>Relative Size & Percentage of Base & $100 \%$ \\
Number of Prism Layers & Number of Prism Layers & 24 \\
Prism Layer Stretching & Prism Layer Stretching & 1.09 \\
Prism Layer Thickness $>$ Relative to Base & Percentage of Base & $100 \%$ \\
Surface Curvature & \# Pts/circle & 36 (default) \\
Surface Growth Rate & Surface Growth Rate & 1.3 (default) \\
Surface Proximity & \# Points in a gap & 2.0 (default) \\
Surface Proximity & Search Floor & 0.0 (default) \\
Surface Size $>$ Relative Minimum Size & Percentage of Base & 25 \\
Surface Size $>$ Relative Target Size & Percentage of Base & $100 \%$ \\
Template Growth Rate & Default Growth Rate & Fast (default) \\
Template Growth Rate & Boundary Growth Rate & None (default) \\
\hline
\end{tabular}

The resulting three-dimensional mesh had 990832 hexahedral cells with a $z_{p}$ of $0.041 \mathrm{~m}$ on the ground and the dune, so that $z_{p}>k_{s}$ as shown in Table 7-2. The three-dimensional mesh was then converted to a two-dimensional mesh by 
removing the thickness, resulting in 17328 cells. This mesh was used for all subsequent simulations and is shown in Figure 7-1. A grid sensitivity analysis was carried out to determine the solution error associated with the discretisation, using the procedure of Celik et al. (2008), which is presented in Appendix E. The mesh thus specified proved to provide acceptable discretization error, based on the reattachment length, velocity and turbulent kinetic energy in the wake of the dune.

Table 7-4: Inputs for mesh refinement

\begin{tabular}{|c|c|}
\hline Property Name & Value \\
\hline Relative/Absolute> Relative to base & $25 \%$ \\
\hline Boundary Growth Rate & None (default) \\
\hline Boundaries/Feature Curves & "Dune" \\
\hline Default Growth Rate & Fast (default) \\
\hline \multicolumn{2}{|c|}{ Wake Refinement Downstream } \\
\hline Distance & $200 \mathrm{~m}$ \\
\hline Direction & {$[1.0,0.0,0.0]$} \\
\hline \multicolumn{2}{|c|}{ Wake Refinement Upstream } \\
\hline Distance & $150 \mathrm{~m}$ \\
\hline Direction & {$[-1.0,0.0,0.0]$} \\
\hline \multicolumn{2}{|c|}{ Wake Refinement Vertically } \\
\hline Distance & $15 \mathrm{~m}$ \\
\hline Direction & {$[0.0,0.0,1.0]$} \\
\hline
\end{tabular}

\subsection{Flow Physics Solution Specification}

The flow physics were chosen to correspond with the flow conditions specified for the ABL profile simulations, under different thermal stratifications, described in Chapter 5. The effect of the thermal stability of the atmosphere on the flow over the dune could therefore be tested.

\subsubsection{Physics models and solvers}

The physics models used were as follows: the fluid space was specified as twodimensional and the time specification was steady. The material specified was air with the same properties as given in Chapter 5, with the density calculated through the ideal gas law. The segregated flow solver was chosen, which achieves pressure-velocity coupling through the SIMPLE algorithm, and solves the total energy equation through the segregated fluid temperature model, which uses temperature as the independent variable (CD-adapco Inc. 2011). Convection of 
momentum and energy was solved using the second-order upwind scheme. The turbulent viscous regime was chosen and the Reynolds-Averaged Navier-Stokes (RANS) turbulence models were specified.

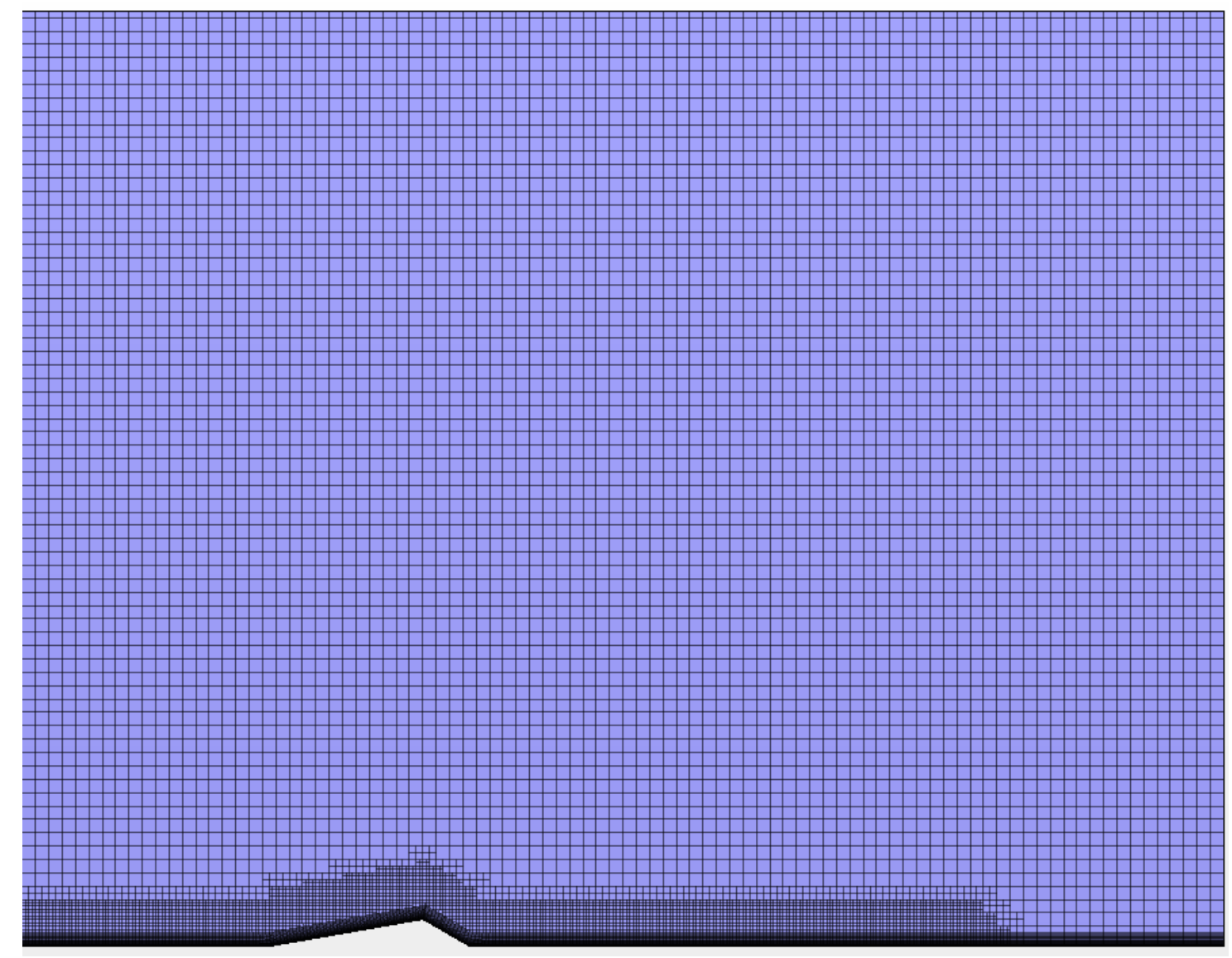

Figure 7-1: Computational mesh for dune simulations

The solver employed the Gauss-Seidel relaxation scheme with under-relaxation factors given in Table 7-5. The solution was run until the normalised residuals of continuity, momentum, energy and turbulence had converged.

Table 7-5:Under-relaxation factors for flow simulations

\begin{tabular}{lc}
\hline Pressure & 0.3 \\
Velocity & 0.7 \\
Energy & 0.9 \\
Turbulence & 0.8 \\
Turbulent Viscosity & 1.0 \\
\hline
\end{tabular}


A reference pressure of $101325 \mathrm{~Pa}$ was used, which is applied by default at the point $[0,0]$ in the fluid domain (CD-adapco Inc. 2011). The flow was initialised using the inlet boundary conditions for velocity, temperature, and turbulence quantities defined below, as well as zero pressure. Furthermore, the models had the momentum and energy source terms accounting for gravity effects specified, as well as the turbulence sources due to gravity, as described in Chapter 5. The turbulence model constants are shown in Table 7-6, with the handling of the $C_{\varepsilon 3}$ parameter specified as described in Chapter 5.

Table 7-6: Turbulence model constants

\begin{tabular}{lcccccccc}
\hline \multirow{2}{*}{ Standard $\boldsymbol{k}-\boldsymbol{\varepsilon}:$} & $C_{\varepsilon 1}$ & $C_{\varepsilon 2}$ & $C_{\mu}$ & $\sigma_{k}$ & $\sigma_{\varepsilon}$ & & & \\
& 1.1523 & 1.92 & 0.0333 & 1.0 & 1.3 & & & \\
\hline \multirow{2}{*}{ SST $\boldsymbol{k}$ - $\boldsymbol{\omega}:$} & $\alpha_{1}$ & $\beta_{1}$ & $\beta_{*}$ & $\sigma_{k 1}$ & $\sigma_{\omega 1}$ & $\beta_{2}$ & $\sigma_{k 2}$ & $\sigma_{\omega 2}$ \\
& 0.31 & 0.075 & 0.09 & 0.85 & 0.5 & 0.0828 & 1.0 & 0.856 \\
\hline
\end{tabular}

\subsubsection{Boundary conditions}

The CFD once again had to contend with separated flow and good grid resolution close to the dune surface and the ground was therefore required. The mesh was generated so that $z_{p}$ was just greater than the value specified for the roughness height $k_{s}$, and thus represented the limit of how close the near wall node can be to the boundary. This treatment yielded an average $z^{+}$value of roughly 500 for the ground and the dune. The high $z^{+}$wall treatment was therefore implemented. The value of the roughness height was specified on the ground, which was modelled as a rough wall with the no-slip shear stress specification, as well as the dune which received the same boundary treatment. Additionally the ground and the dune had the temperature $T_{0}$ from Table 7-2 specified.

The downstream flow outlet was specified as a "flow-split outlet" with a split ratio of one. The "velocity inlet" or inlet boundary upstream of the fluid domain had the static temperature (given by Equation 5-16), velocity components $\left(u_{x}\right.$ equal to $u(z)$ given by Equation 5-15, with zero $u_{y}$ and $u_{z}$ ) and turbulence properties specified using the "Table $(\mathrm{x}, \mathrm{y}, \mathrm{z})$ " method, with the properties calculated in Microsoft Excel and imported to STAR-CCM+. The vertical distributions of $k$ and $\varepsilon$ given by Equations 3-14 and 3-16 were used to specify the inlet turbulence parameters and Equation 3-19 was used to specify the specific dissipation rate $(\omega)$. The pressure is internally calculated from the continuity equation and the density is calculated by the code from the ideal gas law.

The top boundary is where the undisturbed flow conditions were prescribed using the "velocity inlet" boundary condition. This includes the velocity components, the temperature and the turbulence quantities. The values for these quantities for the different thermal stability conditions are given in Table 7-7. The use of this boundary assists in feeding momentum into the flow domain. 
Table 7-7: Flow values specified at top boundary

\begin{tabular}{lccccc}
\hline & {$\left[u_{x}, u_{y}, u_{z}\right](\mathrm{m} / \mathrm{s})$} & $T(\mathrm{~K})$ & $k(\mathrm{~J} / \mathrm{kg})$ & $\varepsilon(\mathrm{J} / \mathrm{kg} . \mathrm{s})$ & $\omega\left(\mathrm{s}^{-1}\right)$ \\
\hline Neutral & {$[11.343,0,0]$} & 294.65 & 1.369 & 0.000892 & 0.0196 \\
Stable & {$[17.181,0,0]$} & 281.31 & 1.187 & 0.004149 & 0.1050 \\
Unstable & {$[9.987,0,0]$} & 301.11 & 9.987 & 0.012296 & 0.0370 \\
\hline
\end{tabular}

\subsection{Comparison of Measurements with CFD Results}

The CFD and experimental results could be compared using hit rates as a quality assessment metric. This quantity indicates the fraction of the $N$ measurement locations where the CFD results are within a $25 \%$ interval of the experimental data (Britter, and Schatzmann 2007). Normalised values are used, with the reference wind speed and dune height used for normalisation.

The distributions of $x$-velocity were normalised with the reference velocity $u_{h}$ and compared with vertical distributions of the wind tunnel data of Liu et al. (2011) at values of $-8 h,-5.6 h,-2.8 h, 0 h, 1.7 h$, and $10 h$, where $0 h$ corresponds to the crest of the dune, $-5.6 h$ to the dune toe on the windward side and $1.7 h$ to the dune toe on the leeward side. The points $1.7 \mathrm{~h}$ and $10 \mathrm{~h}$ are therefore in the wake.

\subsection{Results and Discussion}

The non-dimensional results of the horizontal (streamwise) velocity obtained for the neutral atmosphere using the two different turbulence models are shown in Figure 7-2, along with the non-dimensional measurements from the wind tunnel study. Reasonable agreement is found between the experimental data and the numerical results. The flow acceleration on the upwind slope $(-2.8 h$, Figure 7-2(c)), the pronounced velocity gradient on the dune crest $(0 h$, Figure 7-2(d)) and the flow separation and recirculation in the dune wake $(1.7 \mathrm{~h}$, Figure 7-2(e)) were reproduced by the CFD simulations.

Generally the air flow field of the wind tunnel study is well represented by the CFD models but with noticeable disagreement in the near wall zones at some of the measurement locations. This was also seen by Liu et al. (2011) who attributed it to the limitation of the measuring system which cannot resolve the high-speed gradients in the near-surface zone. It was further observed that the SST $k-\omega$ turbulence model generally performed better than the $k-\varepsilon$ turbulence model, which showed greater acceleration at the dune crest and faster downstream flow recovery than suggested by the measured data. This is reflected in the results of the hit rate analysis presented in Table 7-8, where it can be seen that the SST $k$ - $\omega$ turbulence model better represented the measured data. 


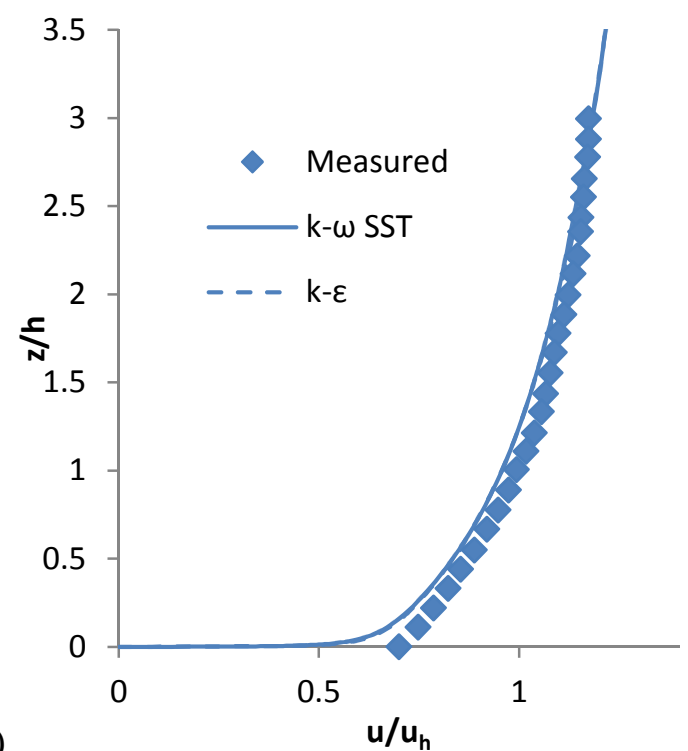

(b)
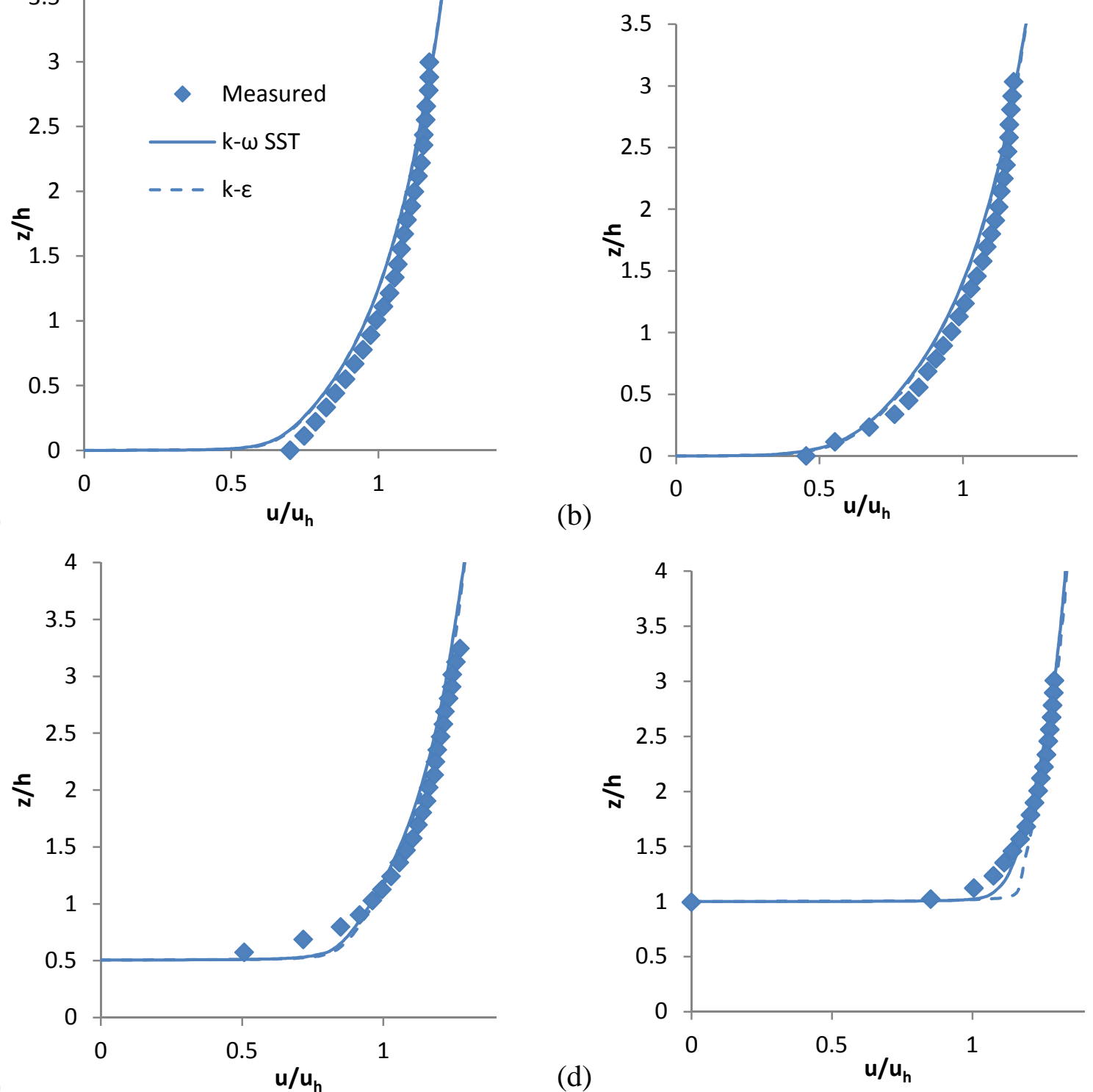

(c)

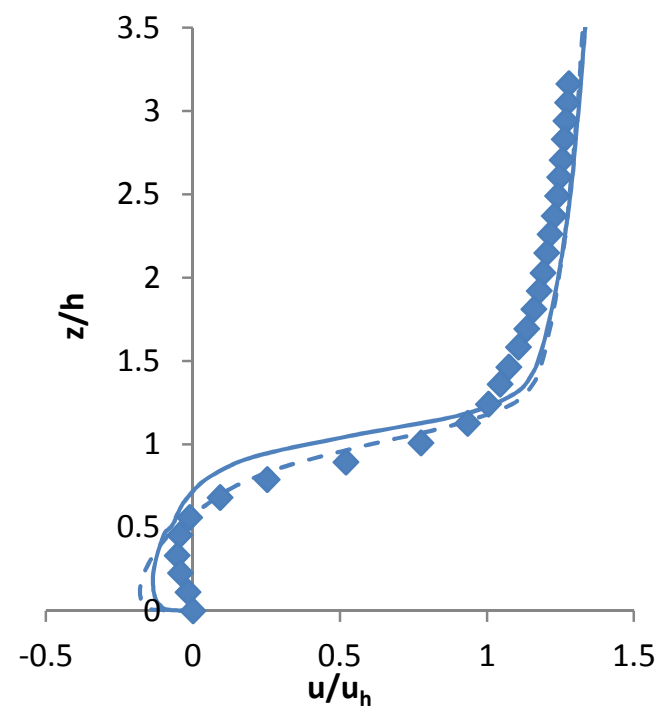

(d)

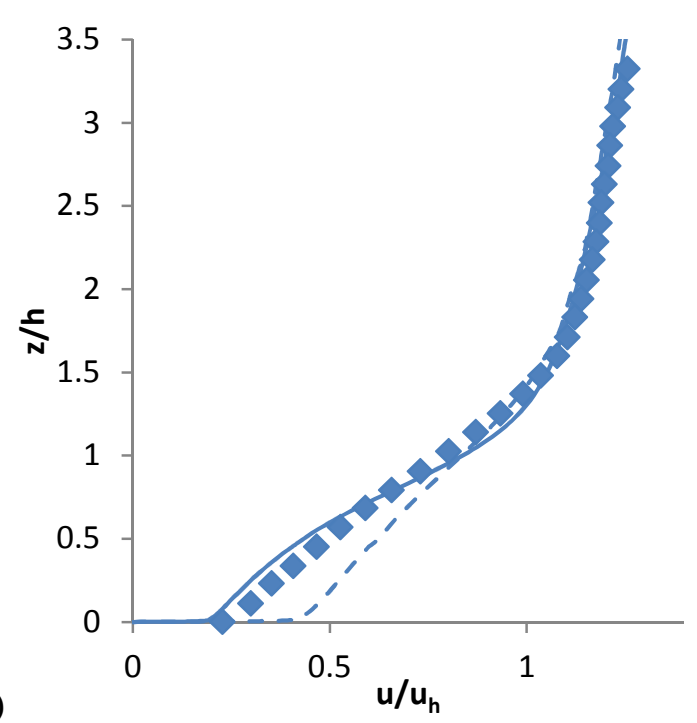

Figure 7-2: CFD horizontal velocity results for the two turbulence models under neutral conditions versus measurement at six locations from $-8 h$ to $10 h$ ((a)-(f)) respectively. 
(a)
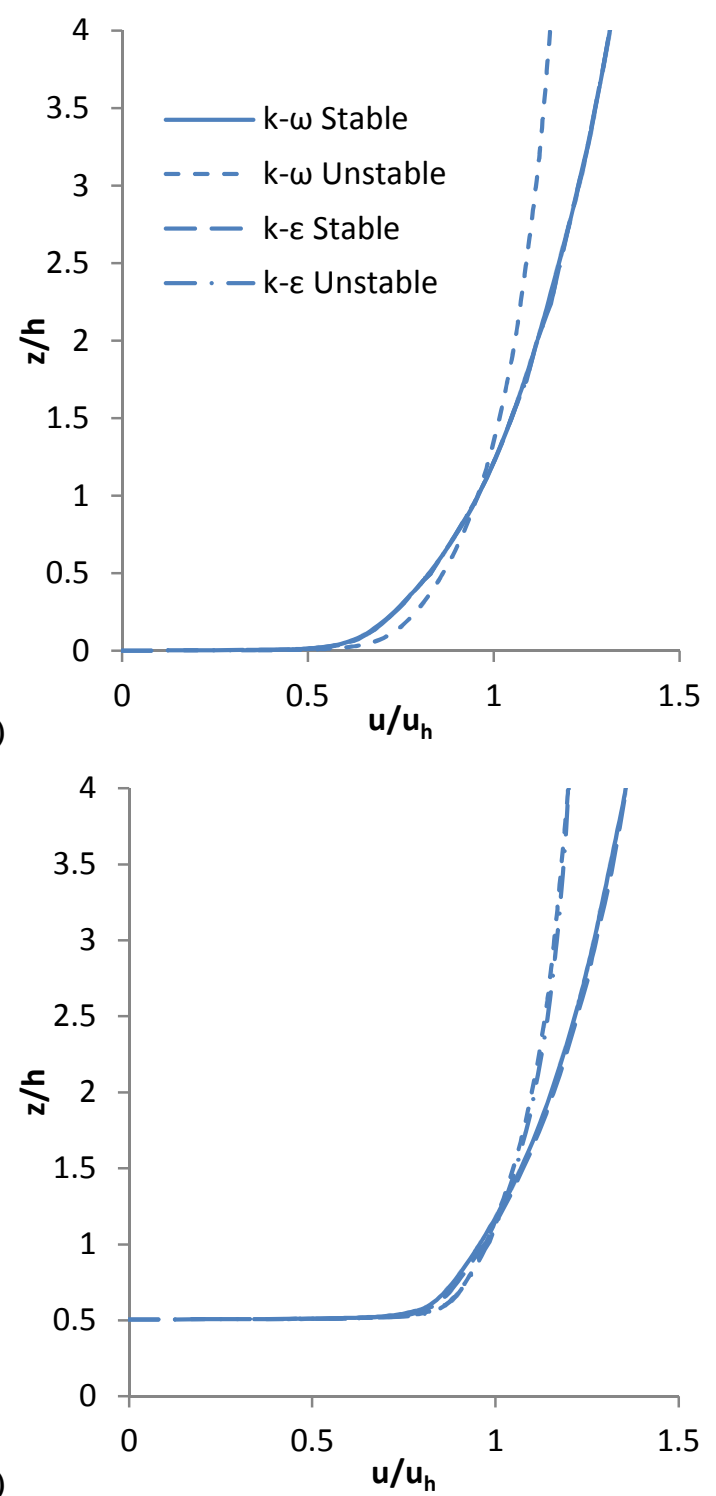

(c)

(d)

(b)
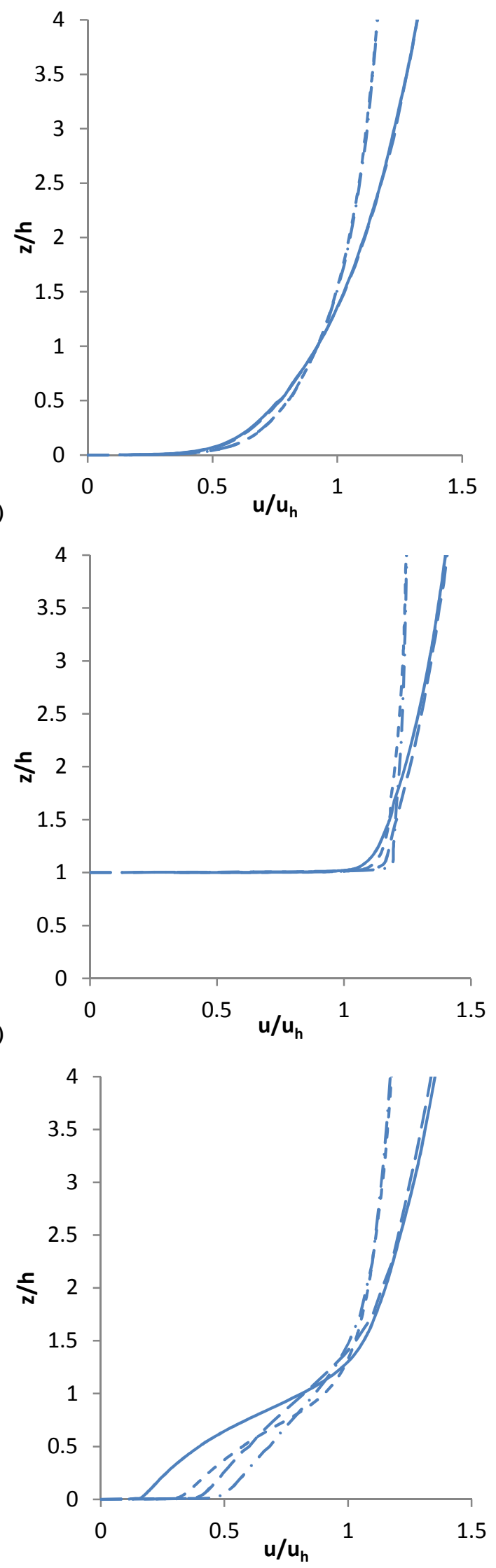

(e)

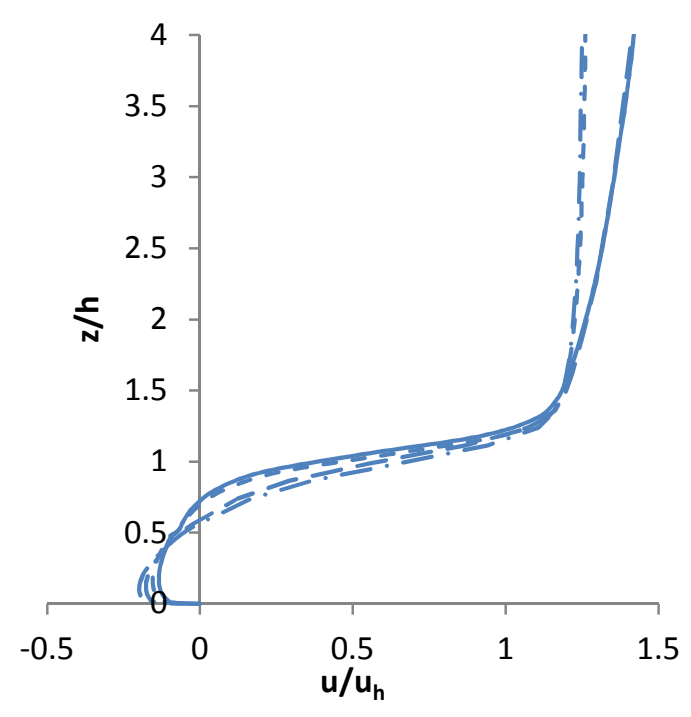

(f)

Figure 7-3: Horizontal velocity distribution for the two turbulence models for stable and unstable conditions at different locations from $-8 h$ to $10 h$ ((a)-(f)) respectively. 
Table 7-8: Results of hit rate analysis with wind tunnel data for neutral atmosphere

\begin{tabular}{lc}
\hline Cases & Hit Rate for Velocity [\%] \\
\hline$k-\omega$ SST & 90.5 \\
Standard $k-\varepsilon$ & 88.6 \\
\hline
\end{tabular}

It was found that the $k$ - $\varepsilon$ turbulence model consistently predicted slightly higher turbulent viscosities than the SST $k-\omega$ turbulence model, illustrated graphically in Appendix G. This was particularly true in the wake region, where it was seen that the $k-\varepsilon$ turbulence model over predicts the flow recovery, attributed to the greater mixing effect brought about by the higher turbulent viscosity. Both turbulence models predicted larger negative velocities in the separation bubble $(1.7 \mathrm{~h}$, Figure 7-2(e)) than those that were measured. This can be ascribed to either the limitation of the measuring system, or the three-dimensional effects present in the wind tunnel that are unaccounted for by the CFD, where Liu et al. (2011) found lateral dispersion of the separation bubble along the leeward face for the threedimensional case.

The horizontal velocity results achieved with the two turbulence models at the various measurement points for the unstable and stable atmospheres are shown in Figure 7-3. Greater flow acceleration on the upwind slope (-2.8h, Figure 7-3(c)) is observed for unstable conditions as well as greater negative velocities in the separation bubble (1.7h, Figure 7-2(e)) and faster flow recovery downstream (10h, Figure 7-2(f)). Again the $k-\varepsilon$ turbulence consistently predicted greater flow acceleration upstream and faster flow recovery downstream of the dune than the SST $k$ - $\omega$ turbulence model, for the thermally stratified atmosphere.

These observations can again be explained by the higher turbulent viscosities predicted by the $k$ - $\varepsilon$ turbulence model and the higher turbulent viscosities present in the unstable atmosphere (shown in Appendix G), where higher turbulent viscosity leads to increased mixing in the flow and therefore faster flow recovery in the wake. These results are qualitatively similar to the wind tunnel observations of thermally stratified flow over a hill reported by Takahashi et al. (2005). This effect too is partially reflected in the calculation of re-attachment length, where the shear stress on the wall is zero, for the different thermally stratified atmospheres, shown in Table 7-9. Flow re-attachment takes longer in the stable case, followed by the neutral case, with the reattachment happening considerably earlier for the unstable atmosphere. These results for re-attachment length are also consistent with previous flow simulations for idealised transverse dunes under neutral conditions (Parsons et al. 2004a, b).

The validity of comparing the scaled results of a wind tunnel study with a full scale simulation will now be addressed. Wind tunnel models are generally Reynolds number dependent, but at certain levels of wind speed and model size a critical Reynolds number is reached, after which Reynolds number independence is attained. At this point, for increased flow velocity and model size, the nondimensional flow structure becomes independent of scale and the results can be 
considered representative of the full scale case (Uehara et al. 2003). The critical Reynolds number, however, depends of the size and shape of the model.

Table 7-9: Flow re-attachment lengths for different stability conditions

\begin{tabular}{lcc}
\hline & \multicolumn{2}{c}{ Length to flow re-attachment } \\
\hline & {$[\mathrm{m}]$} & $(x / h)$ \\
\hline Neutral & 80.625 & 8.0625 \\
Stable & 81.875 & 8.1875 \\
Unstable & 70.625 & 7.0625 \\
\hline
\end{tabular}

A critical Reynolds number of $9 \times 10^{4}$ has been reported for cosine shaped hills (Ferreira et al. 1995), and values ranging from 4000 to 7500 for block-shaped obstacles (Uehara et al. 2003). In addition Wiggs et al. (1996) found good agreement between the non-dimensional results of flow over a scaled dune model in a wind tunnel (Reynolds number $3.2 \times 10^{3}$ ), and full scale field measurements over a dune (Reynolds number $4.4 \times 10^{6}$ ), provided that similarity existed for the velocity profiles. This is similar to the case observed in this investigation, with a wind tunnel model that yields a Reynolds number of $1.1 \times 10^{4}$, and the full scale CFD model with a Reynolds number of $4.6 \times 10^{6}$. This suggests that the nondimensional results of the wind tunnel and CFD are comparable, as the wind velocity profile used in the CFD was chosen to display similarity with the one measured in the wind tunnel. Furthermore it has been suggested that roughness Reynolds number $\left(\rho u_{*} z_{0} / \mu\right)$ can be used as gauge of Reynolds number independence, with a critical value of 2.5 being proposed (Uehara et al. 2003). The wind tunnel study of Liu et al. (2011) had a $R e_{z 0}$ of 3.3, again suggesting that the non-dimensional results of this particular wind tunnel study can be applied to a full scale model.

The isovel contour plots of the streamwise velocity for the different thermally stratified atmospheres are shown in Figure 7-4. The model is seen to predict flow deceleration immediately upwind of the dune, followed by windward slope acceleration with a maximum velocity at the crest, and then negative flow in the separation bubble. Another observation is the convergence in the faster upper isovels of the flow fields, which corresponds to a zone of "jet" of accelerated, overshot flow which extends from the crest above the flow separation bubble (Parsons et al. 2004a). These results are consistent with those of other dune flow studies (Parsons et al. 2004a, b, Livingstone et al. 2007). It is clear that this overshot flow is less pronounced in the stable flow case (Figure 7-4 (b)) and more pronounced in the unstable case (Figure 7-4 (c)). This is consistent with our understanding of the behaviour of flow under different thermal stratifications. One expects that flow that is vertically displaced by an obstacle will be decelerated by buoyancy in the stable atmosphere, while buoyancy forces will accelerate the flow displaced vertically in an unstable atmosphere. This results in greater acceleration of the flow above the dune crest in the unstable case, and less flow acceleration in the stable case. 


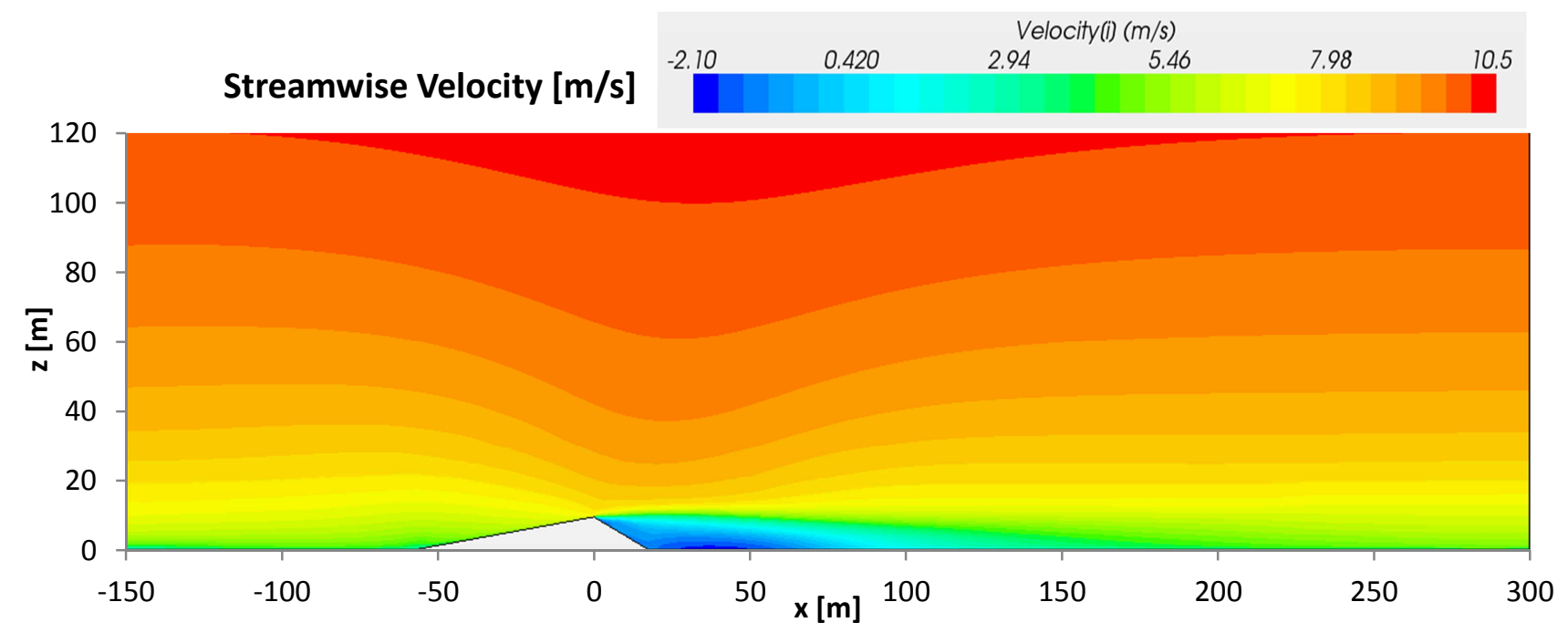

(a)

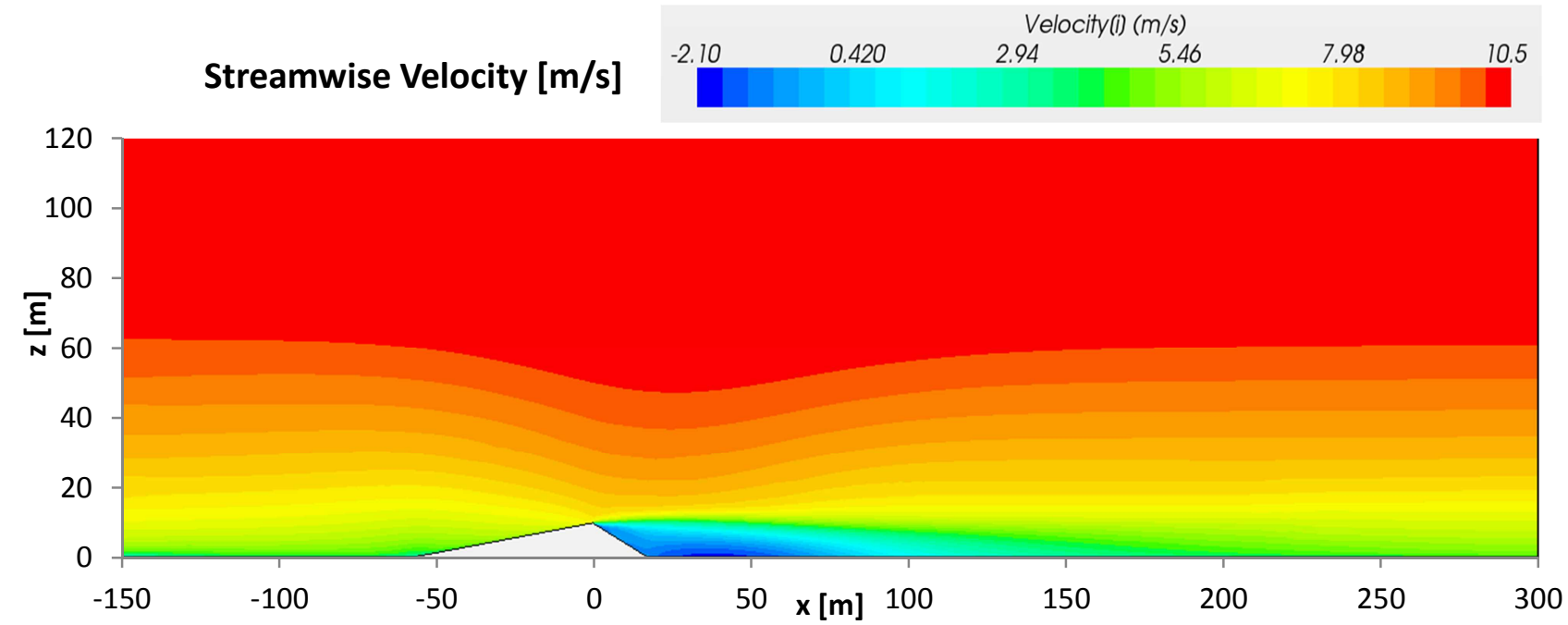

(b)

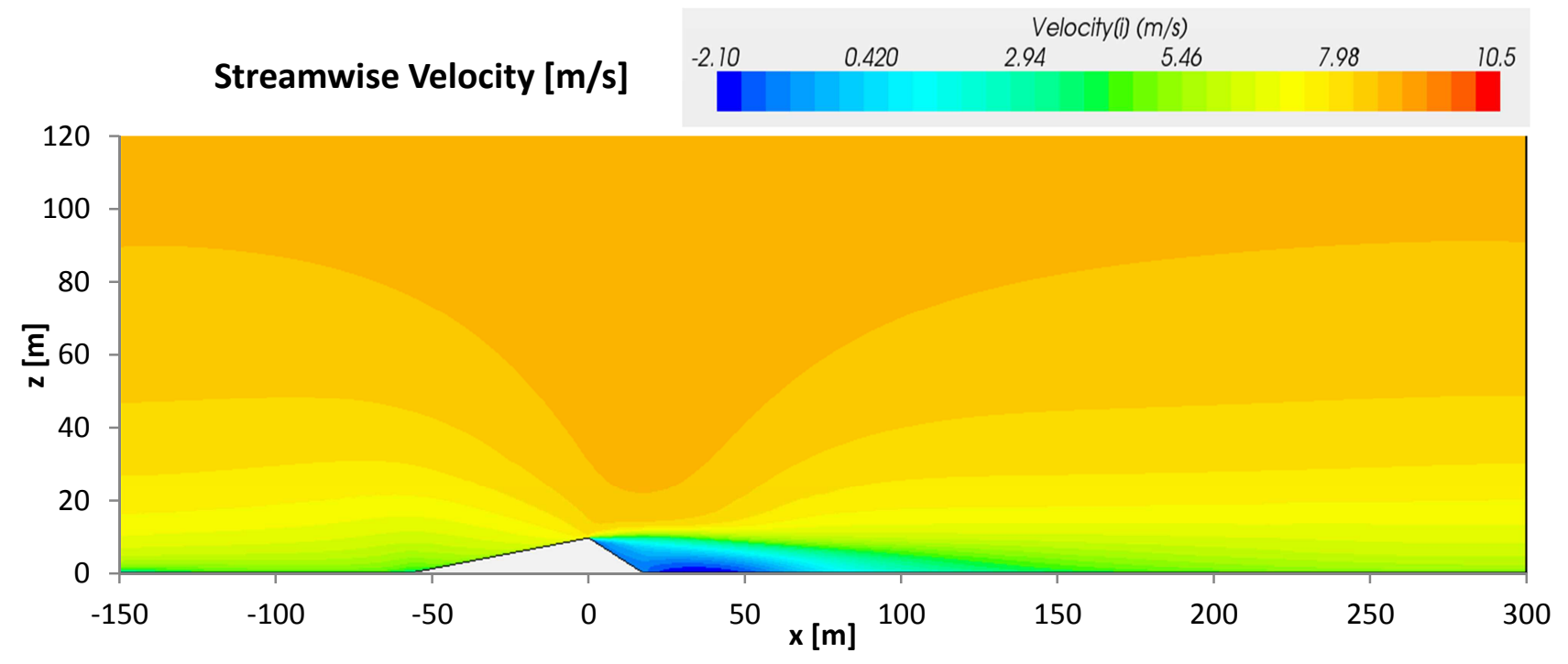

(c)

Figure 7-4: Contour plots of horizontal (streamwise) velocity for (a) neutral conditions; (b) stable conditions and; (c) unstable conditions 

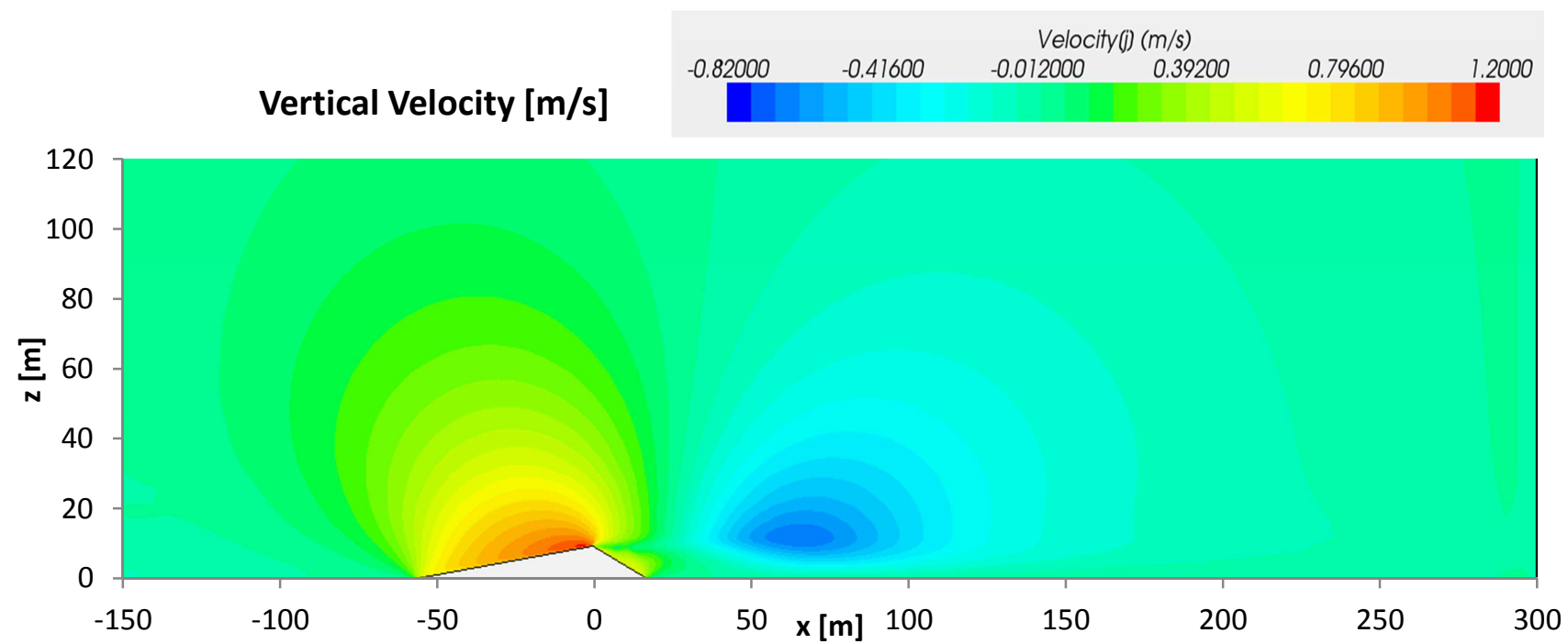

(a)
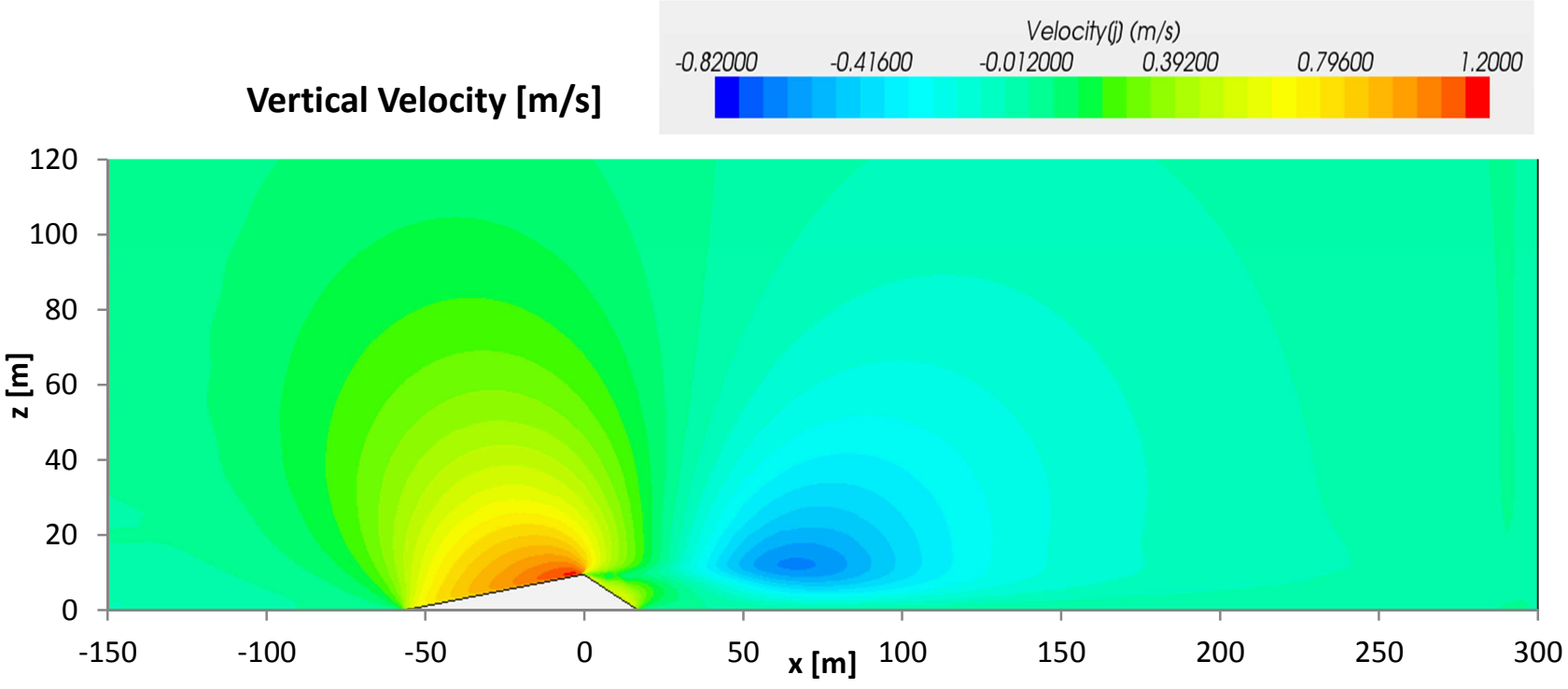

(b)
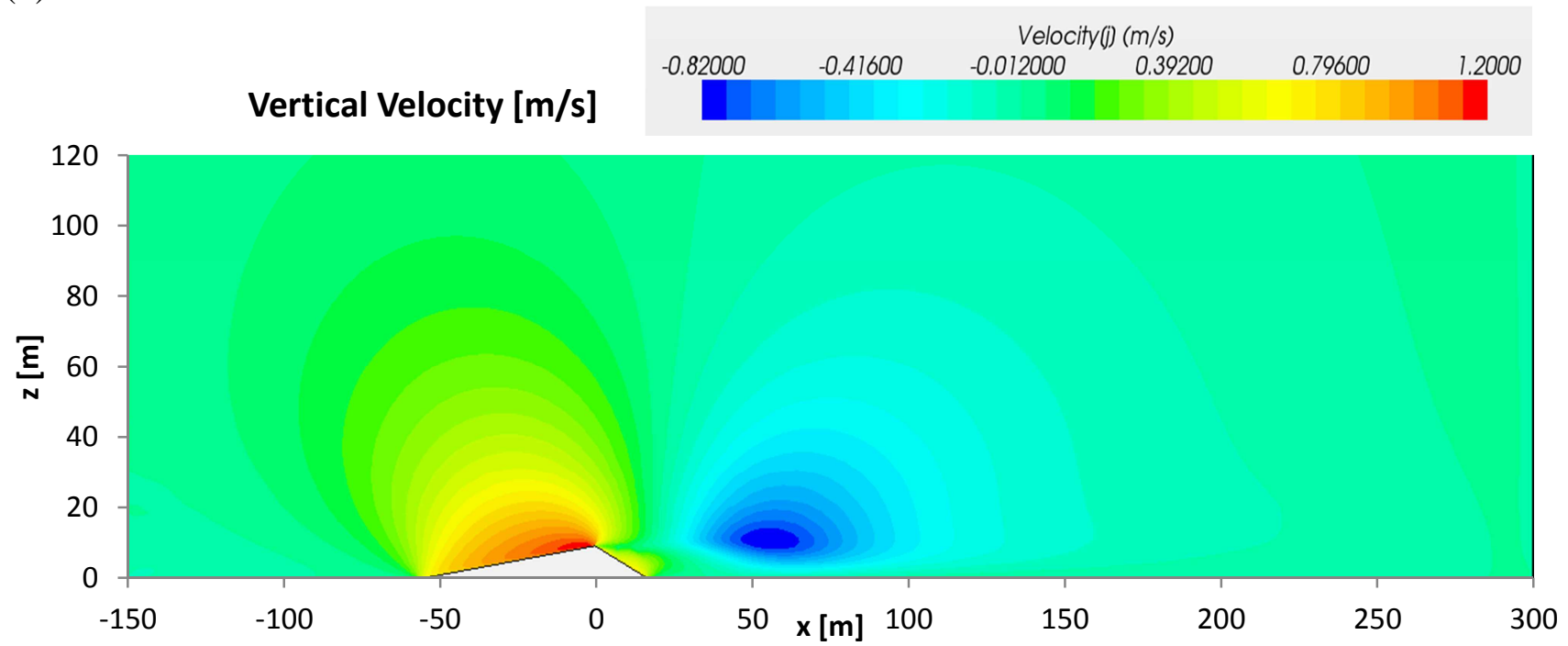

(c)

Figure 7-5: Contour plots of vertical velocity for (a) neutral conditions; (b) stable conditions and; (c) unstable conditions 
Figure 7-5 shows the vertical velocity field for the different thermally stratified atmospheres. A zone of positive upward flow exists on the windward slope and reaches a maximum at the crest, which is related to the upward forcing effect of the dune. Small pockets of upward moving air is also seen on the leeward slope as the recirculating zone drives the air up the dune face. A zone of strongly downward moving air exists in the lee and extends above the flow separation zone from the base of the leeward slope to beyond the flow re-attachment point. This too is consistent with previous observations (Livingstone et al. 2007, Parsons et al. 2004a, b).

As expected for the stable atmosphere (Figure 7-5(b)) the upward and downward vertical velocities are less pronounced than in the other two cases. A greater upward velocity is seen on the windward side and a greater negative velocity is seen on the leeward side in the unstable case (Figure 7-5(c)). This is once again explained by the physics of the thermally stratified atmosphere where a vertically displaced air parcel will be decelerated in the stable atmosphere and accelerated upwards or downwards in the unstable atmosphere. This larger negative vertical velocity also helps to explain the lower re-attachment length seen for the unstable case, while the stable atmosphere's smaller negative velocity contributes to the longer re-attachment length. A larger zone of upward moving air is also seen on the lee side of the unstable case compared to the neutral and stable cases.

It should therefore be clear that the thermal stratification of the atmosphere can have a significant effect of the flow field over a sand dune. This has implications for the prediction of sand and seed transport which influences the dynamics of desert geomorphology, and it can also have an effect on the overall dune geometry. The CFD study of these kinds of structures cannot therefore be limited to the neutral atmosphere as is was shown in Chapter 4 that the thermally stratified atmosphere is more prevalent in desert areas, with stable conditions predominating at night and unstable condition predominating during the day.

It was shown that the flow field over a dune is potentially greatly influenced by the thermal stability of the atmosphere and this is surely true for other structures immersed in the thermally stratified ABL. With an increasing emphasis being placed on renewable energy, accurate wind resource prediction models are required. As was shown in Chapter 5, the ABL becomes increasingly more influenced by the thermal stability condition with increasing height. This can lead to discrepancies in the wind speed predictions which could result in less than expected power outputs from wind turbines, particularly in areas where unstable conditions predominate. The prediction of thermal stability has implications on pollution dispersion models as well, where the stable atmosphere could lead to the build-up of particulates in the lower atmosphere. The accurate CFD modelling of the thermally stratified atmosphere is therefore very important in wind studies, as it has been shown here that the assumption of neutral conditions could lead to an incomplete picture of the flow conditions that influence any particular case of interest. For airflow over desert sand dunes the influence of the thermal stability condition of the atmosphere has been found to play a significant role. 


\section{CONCLUSIONS}

It has been shown in this study that velocity and temperature profiles under the influence of thermal stability can readily be measured, and were found to be prevalent in the interdune area of the Namib Desert. Thermally stable conditions were found to occur at night and unstable conditions were found during the day, with no instance of the neutral atmosphere found during the measurement period at the site. It was established that these velocity and temperature profiles could be described by Monin-Obukhov similarity theory which would lead to the determination of the roughness length, surface shear stress and heat flux, as well as the Richardson number and Monin-Obukhov length which describe the thermal condition of the ABL. More accurate profile extrapolations, based on MoninObukhov similarity theory, could therefore be made.

A procedure for simulating the thermally stratified ABL using CFD was also described. In each case the numerical solution was found to be stable with good levels of solution convergence. The simulation demonstrated the capability of maintaining the temperature, velocity and turbulence profiles along an extensive downstream flow distance, therefore displaying high levels of horizontal homogeneity, a precondition for accurate CFD wind model predictions. The CFD model further demonstrated the capability of accounting for buoyancy and gravity effects, by allowing for the full variation of density, temperature and pressure. It should be noted however that this study is not intended to be an exhaustive analysis of the reconciliation of atmospheric models with CFD equation sets, and further research in this regard is proposed. A comprehensive study of the CFD modelling of the adiabatic lapse rate and the reconciliation of the CFD energy equation with atmospheric models would be of particular interest.

In each case tested, the hydrostatic pressure, and the density variation that it brings about, developed in the flow field by the addition of the fluid weight into the momentum equation, thus also introducing buoyancy forces. It was further found that gravity effects could be introduced into several two-equation RANS turbulence models, which displayed the advantage of lower computational requirement, leading to higher simulation turnover than for other turbulence models like DES. The turbulence models thus modified displayed decent performance in simulating separated regions in the flow field, with the SST $k-\omega$ turbulence model consistently providing results that best aligned to measured experimental data.

Furthermore, it was shown that the thermal stability of the atmosphere can have significant effects on the flow field over a sand dune. It was found that the flow recovery was enhanced and re-attachment occurred earlier in unstable conditions. Flow recovery and re-attachment took longer in stable conditions. It was also found that flow acceleration over the crest of the dune was greater under unstable conditions and that the effect of the dune on the flow higher up in the atmosphere was felt at much higher distances. Under stable conditions, however, the influence 
on the flow higher up in the atmosphere was much less than for unstable or neutral conditions.

Vertical velocities were also enhanced for a dune immersed in the unstable ABL and reduced for stable conditions. The accurate CFD modelling of the thermally stratified atmosphere is therefore very important in wind studies, as it has been shown here that the assumption of neutral conditions could lead to an incomplete picture of the flow conditions that influence any particular case of interest

A literature review of the thermally stratified atmospheric boundary layer, described by Monin-Obukhov similarity theory, and its application to CFD has therefore been provided. This included a short review of the application of CFD to flow involving aeolian geomorphology. Methods for the empirical determination of the thermally stratified atmosphere were described along with ways to parameterise it. A CFD method for modelling the turbulent ABL under different thermal stability conditions was developed and deployed. The case of thermally stratified flow over and idealised transverse dune was investigated and it was found that for airflow over such structures the influence of the thermal stability condition played a significant role. 


\section{APPENDIX A: MATLAB CODE FOR DETERMINATION OF SURFACE FLUXES FROM WIND MAST DATA}

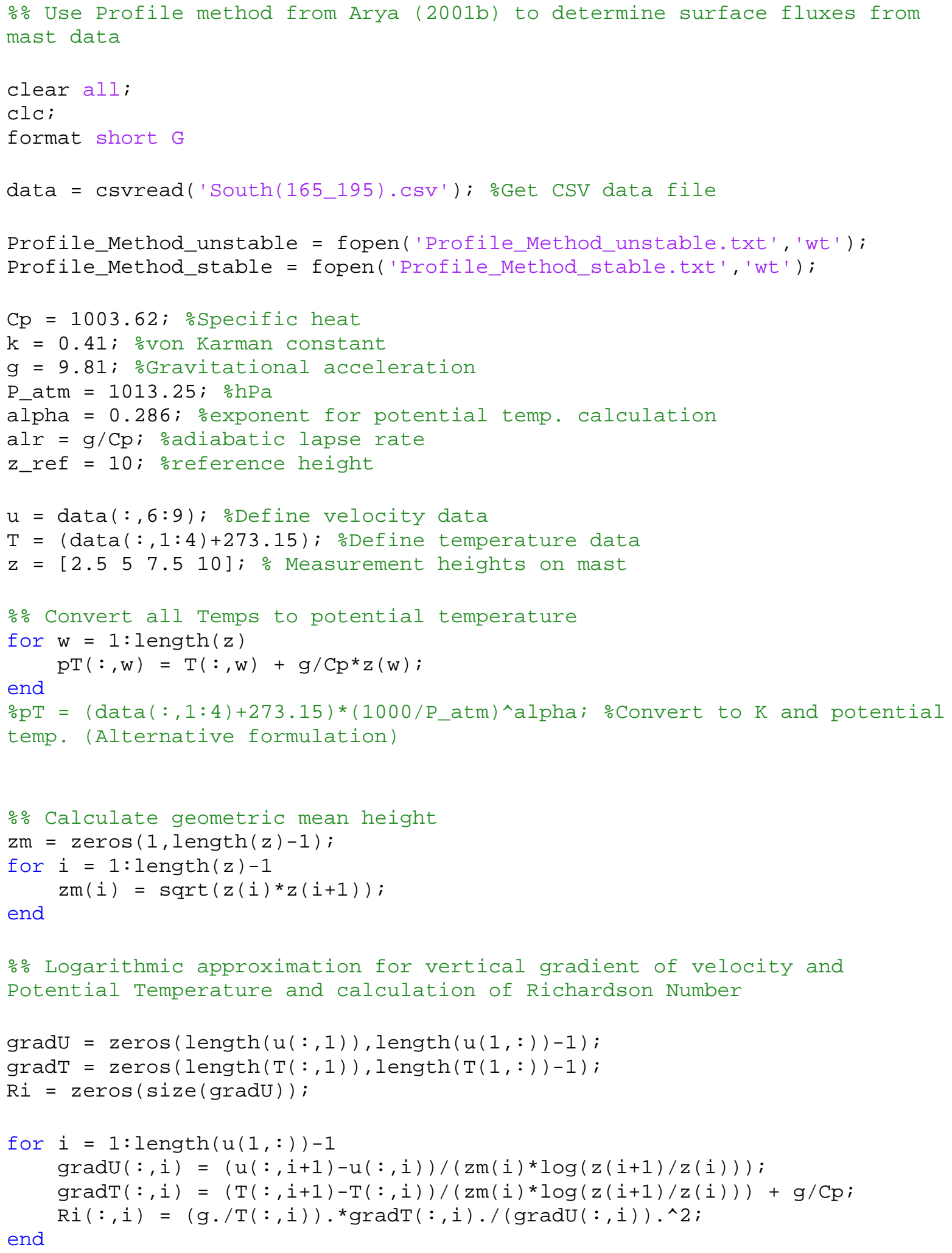




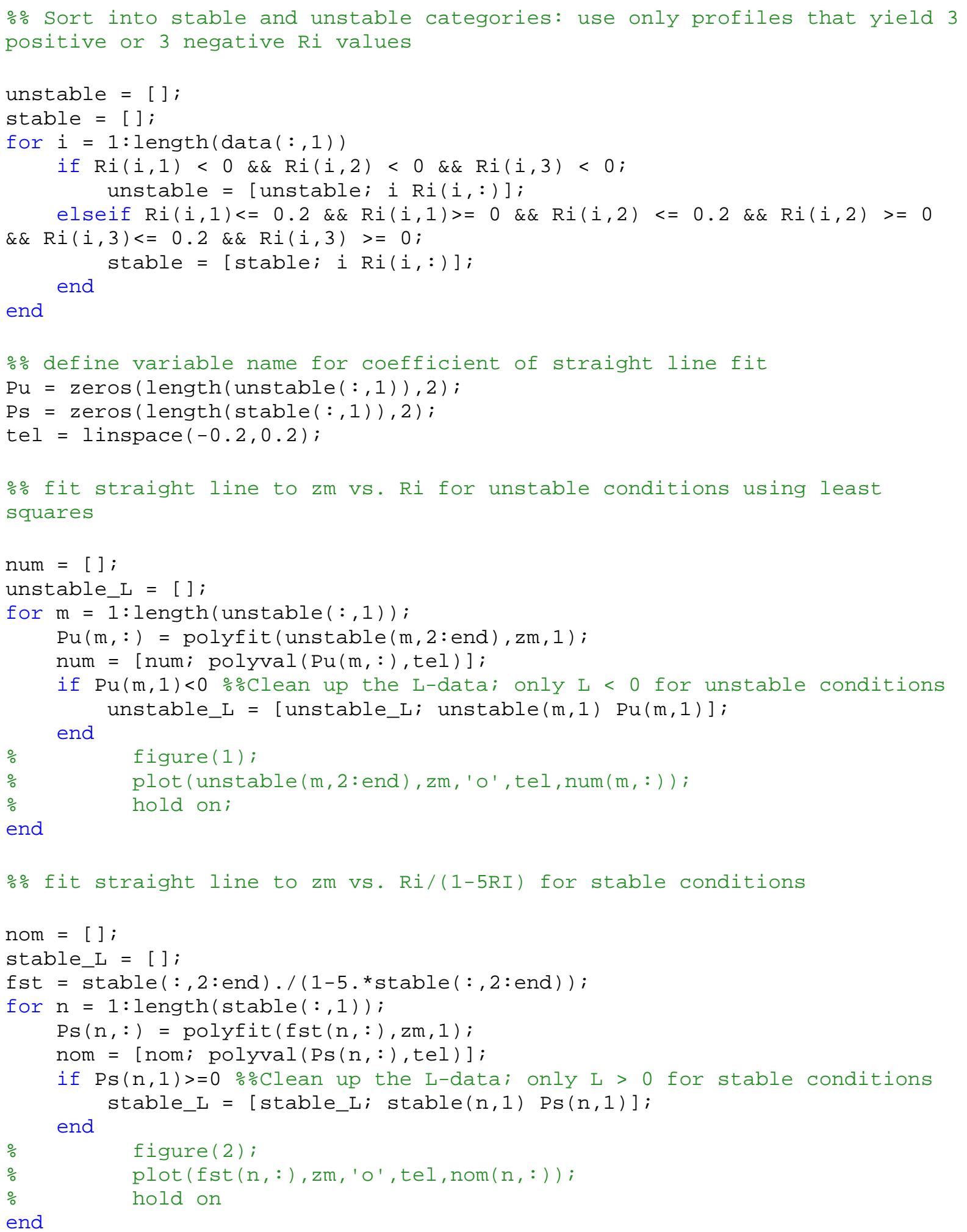

응 Reconcile data and potential temperatures

stable_data $=\left[\operatorname{stable\_ L}(:, 1)\right.$ data $\left(\operatorname{stable\_ L}(:, 1),:\right)$ stable_L $\left.(:, 2)\right]$; unstable_data $=[$ unstable_L $(:, 1)$ data (unstable_L $(:, 1),:$ ) unstable_L $(:, 2)]$;

pT_stable $=$ pT (stable_L $(:, 1),:$ );

pT_unstable $=$ pT (unstable_L $(:, 1),:$ ); 


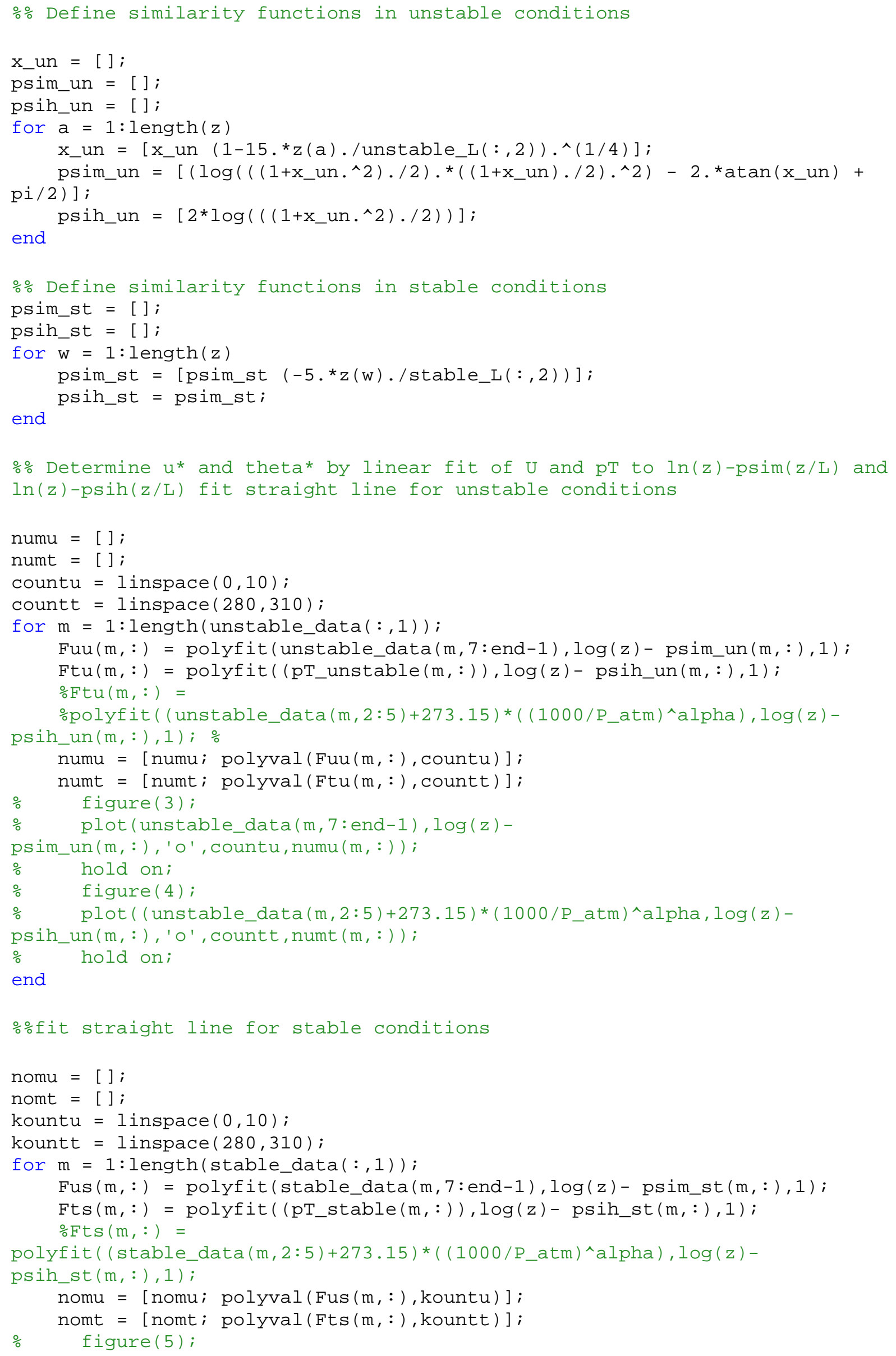




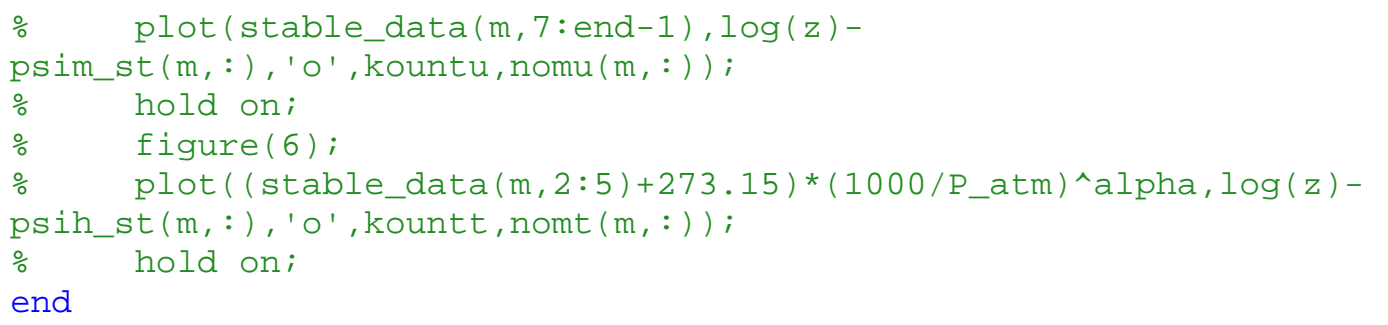

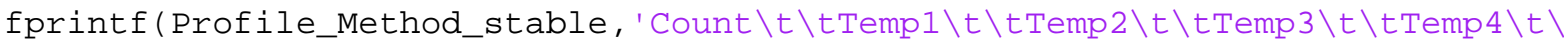
$t D i r \backslash t \backslash t \backslash t V e l 1 \backslash t \backslash t V e 12 \backslash t \backslash t V e l 3 \backslash t \backslash t V e l 4 \backslash t \backslash t L \backslash t \backslash t \backslash t u^{*} \backslash t \backslash t \backslash t T^{*} \backslash t \backslash t \backslash t z 0 \backslash t \backslash t \backslash t T 0$ $\left.\backslash t \backslash t \backslash t H 0 \backslash t \backslash t \backslash t m \backslash n^{\prime}\right) ;$

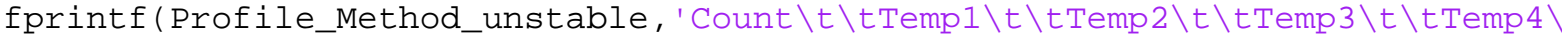
$t \backslash t \operatorname{Dir} \backslash t \backslash t \backslash t \operatorname{Vel} \overline{1} \backslash t \backslash t \operatorname{Ve} \bar{l} 2 \backslash t \backslash t \operatorname{Vel} 3 \backslash t \backslash t \operatorname{Vel} 4 \backslash t \backslash t L \backslash t \backslash t \backslash t u^{*} \backslash t \backslash t \backslash t T^{*} \backslash t \backslash t \backslash t z 0 \backslash t \backslash t \backslash t$ $\left.T 0 \backslash t \backslash t \backslash t H 0 \backslash t \backslash t \backslash t m \backslash n^{\prime}\right)$;

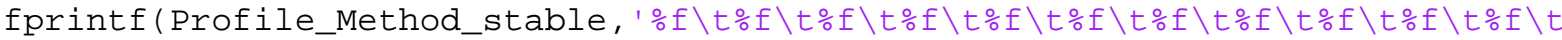
$\circ f \backslash t \div f \backslash t \circ f \backslash t \div f \backslash t \div f \backslash t \circ f \backslash n^{\prime}$, output_stable');

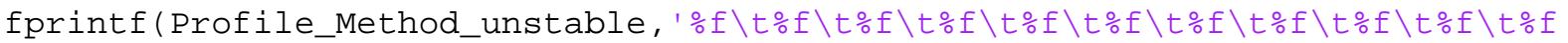
$\backslash t \% f \backslash t \div f \backslash t \% f \backslash t \% f \backslash t \circ f \backslash t \% f \backslash n^{\prime}$, output_unstable'); 


\section{APPENDIX B: WIND PROFILE DATA SAMPLE CALCULATION}

Here follows an illustration of the method of calculating the Monin-Obukhov flux profile relationships from measured temperature and velocity data at different vertical heights. The measurements to be tested are given in Table B-1. For the purpose of illustration one of the profiles has been selected to represent the unstable atmosphere and the other to represent the stable atmosphere. The measurements were taken at heights of $2.5 \mathrm{~m}, 5.0 \mathrm{~m}, 7.5 \mathrm{~m}$ and $10 \mathrm{~m}$ (corresponding to measurement heights $z_{1}, z_{2}, z_{3}$ and $z_{4}$ respectively). The temperature (given in ${ }^{\circ} \mathrm{C}$ ) and velocity (given in $\mathrm{m} / \mathrm{s}$ ) profiles have $T_{1}$ and $u_{1}$ respectively corresponding to the measurement height $z_{1}$, with $z_{2}>z_{1}$.

Table B-1: Mean temperature and velocity data at different measurement heights.

\begin{tabular}{cccccccc}
\hline $\boldsymbol{T}_{\mathbf{1}}$ & $\boldsymbol{T}_{\mathbf{2}}$ & $\boldsymbol{T}_{\mathbf{3}}$ & $\boldsymbol{T}_{\mathbf{4}}$ & $\boldsymbol{u}_{\mathbf{1}}$ & $\boldsymbol{u}_{\mathbf{2}}$ & $\boldsymbol{u}_{\mathbf{3}}$ & $\boldsymbol{u}_{\mathbf{4}}$ \\
\hline 28.93 & 28.76 & 28.65 & 28.58 & 3.85 & 4.45 & 4.78 & 5.00 \\
15.21 & 15.23 & 15.23 & 15.22 & 3.69 & 4.33 & 4.72 & 5.00 \\
\hline
\end{tabular}

First we calculate the geometric mean heights by:

$$
z_{m}=\left(z_{1} z_{2}\right)^{1 / 2}
$$

Therefore:

$$
\begin{aligned}
& z_{m 1}=(2.5 \times 5)^{1 / 2}=3.5355 \mathrm{~m} \\
& z_{m 2}=(5 \times 7.5)^{1 / 2}=6.1237 \mathrm{~m} \\
& z_{m 3}=(7.5 \times 10)^{1 / 2}=8.6603 \mathrm{~m}
\end{aligned}
$$

We must also convert temperature into potential temperature by:

$$
\theta=T+\Gamma z
$$

Therefore for the first temperature measurement:

$$
\theta_{1}=T_{1}-\frac{g}{c_{p}} z_{1}=(28.93+273.15)-0.009775(2.5)=302.1 \mathrm{~K}
$$

The rest of the potential temperatures are given in Table B-2. We can now use the logarithmic finite difference approximation to calculate the velocity and potential temperature gradients, so that the Richardson number can be calculated.

Table B-2: Potential temperatures at each measurement height

\begin{tabular}{cccc}
\hline $\boldsymbol{\theta}_{\mathbf{1}}$ & $\boldsymbol{\theta}_{\mathbf{2}}$ & $\boldsymbol{\theta}_{\mathbf{3}}$ & $\boldsymbol{\theta}_{\mathbf{4}}$ \\
\hline 302.10 & 301.95 & 301.88 & 301.83 \\
288.39 & 288.43 & 288.45 & 288.47 \\
\hline
\end{tabular}


For the first pair of temperature and velocity readings at the first geometric mean height:

$$
\frac{\partial u}{\partial z}=\frac{1}{z_{m}} \frac{\Delta u}{\ln \left(z_{2} / z_{1}\right)}=\frac{(4.45-3.85)}{3.54 \ln (5 / 2.5)}=0.2425 \mathrm{~s}^{-1}
$$

And:

$$
\begin{aligned}
\frac{\partial \theta}{\partial z}=\frac{\partial T}{\partial z}+\Gamma & =\frac{1}{z_{m}} \frac{\Delta T}{\ln \left(z_{2} / z_{1}\right)}+\frac{g}{c_{p}}=\frac{(301.91-302.08)}{3.54 \ln (5 / 2.5)}+\frac{9.81}{1003.62} \\
& =-0.609 \mathrm{~K} \mathrm{~m}^{-1}
\end{aligned}
$$

Therefore the Richardson number at the first geometric mean height for the first set of temperature and velocity measurements is:

$$
R i_{m}=\frac{g}{T_{1}} \frac{(\partial \theta / \partial z)_{z_{m}}}{(\partial u / \partial z)_{z_{m}}^{2}}=\frac{9.81}{302.08}\left(\frac{-0.609}{0.2425}\right)=-0.0336
$$

We notice that the Richardson number is negative and therefore the atmosphere in this layer is unstable. The rest of the Richardson numbers at each geometric mean height for the two profiles are given by are given in Table B-3.

Table B-3: Richardson numbers for the two profiles

\begin{tabular}{ccc}
\hline $\boldsymbol{R} \boldsymbol{i}_{\boldsymbol{m} \mathbf{1}}$ & $\boldsymbol{R} \boldsymbol{i}_{\boldsymbol{m} \mathbf{2}}$ & $\boldsymbol{R \boldsymbol { i } _ { \boldsymbol { m } \mathbf { 3 } }}$ \\
\hline-0.0336 & -0.0582 & -0.0823 \\
0.00819 & 0.0138 & 0.0191 \\
\hline
\end{tabular}

Inspection of Table B-3 reveals that the first set of measurements yields Richardson numbers at each layer between the measurement levels that are negative. This suggests that the atmosphere was unstable when these measurements were made. On the other hand all the Richardson numbers calculated for the other profile are positive, suggesting a stable atmosphere. An estimate of the Monin-Obukhov length $L$ can be obtained from a straight line fit through the data points of $z_{m}$ versus $R i$ for unstable conditions, or $z_{m}$ versus $R i /(1-5 R i)$ for stable conditions, using least squares. In both cases, according to Equations 2-37 and 2-38, $L$ will be equal to the slope of the best fitted line. The plots of the unstable and stable cases are shown in Figure B-1 and Figure B-2 respectively. The straight line fit through the unstable atmosphere data yields:

$$
z_{m}=-105.3 R i
$$

And through the stable atmosphere data:

$$
z_{m}=408.57[R i /(1-5 R i)]
$$




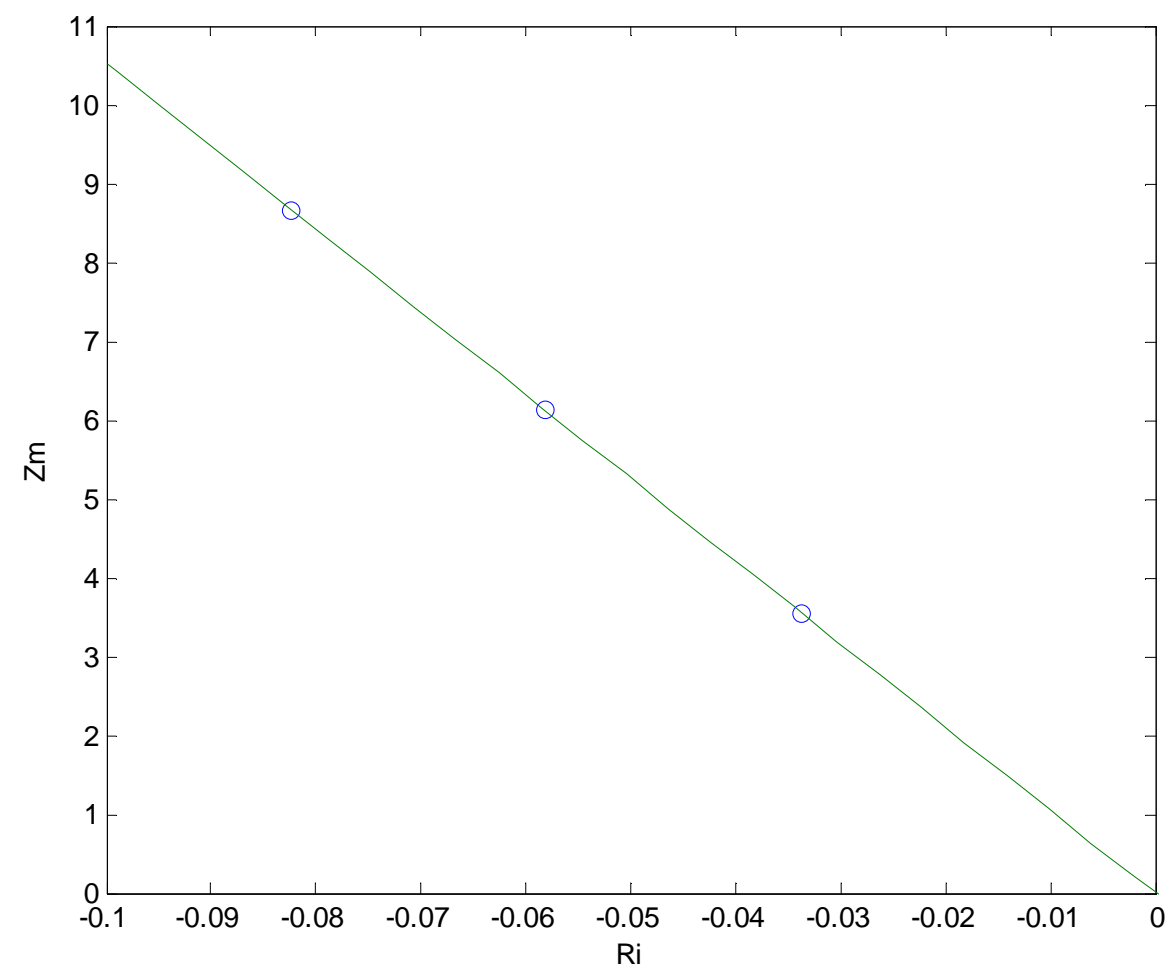

Figure B-1: Least square fitting of unstable Richardson number function versus geometric mean height

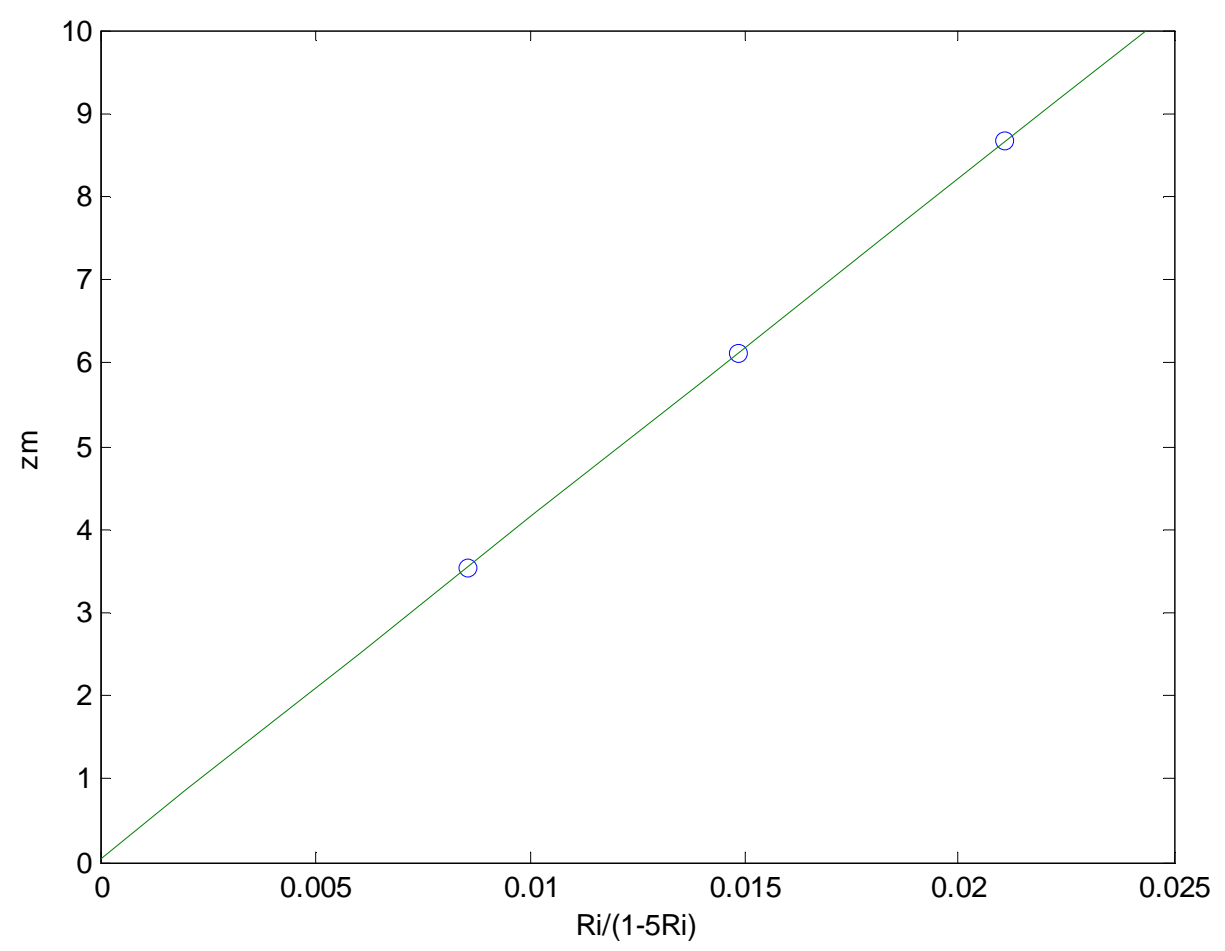

Figure B-2: Least square fitting of stable Richardson number function versus geometric mean height 
Equations B-3 and B-4 therefore suggest a value of $L$ of -105.3 for the unstable case and 408.57 for the stable case.

The next step is to plot $u$ versus $\ln (z)-\psi_{m}(z / L)$ and $\theta$ versus $\ln (z)-\psi_{h}(z / L)$ and to again use least squares to fit a straight line through the data points. According to Equations 2-39 and 2-40, the slopes of these lines must be $\kappa / u_{*}$ and $\kappa / \theta_{*}$, from which $u_{*}$ and $\theta_{*}$ can be determined. The plots for temperature are shown in Figure B-3 for the unstable case and Figure B-4 for the stable case, while the plots for velocity are shown in Figure B-5 for the unstable case and Figure B-6 for the stable case. The straight line fits of these lines yield for the unstable case:

$$
\begin{gathered}
u=1.052\left(\ln (z)-\psi_{m}(z / L)\right)-3.2169 \\
\theta=-3.7924\left(\ln (z)-\psi_{h}(z / L)\right)+1146.5
\end{gathered}
$$

Therefore:

$$
\begin{gathered}
u_{*}=\frac{0.41}{1.052}=0.3897 \mathrm{~m} / \mathrm{s} \\
\theta_{*}=\frac{0.41}{-3.7924}=-0.1081 \mathrm{~K}
\end{gathered}
$$

And for the stable case:

$$
\begin{aligned}
& u=1.1267\left(\ln (z)-\psi_{m}(z / L)\right)-3.2186 \\
& \theta=17.701\left(\ln (z)-\psi_{h}(z / L)\right)-5103.6
\end{aligned}
$$

Therefore:

$$
\begin{aligned}
& u_{*}=\frac{0.41}{1.1267}=0.3632 \mathrm{~m} / \mathrm{s} \\
& \theta_{*}=\frac{0.41}{-3.7924}=0.0232 \mathrm{~K}
\end{aligned}
$$

By rewriting Equations 2-39 and 2-40 in the following forms:

$$
\begin{gathered}
\ln (z)-\psi_{m}(z / L)=\frac{\kappa}{u_{*}} u+\ln \left(z_{0}\right) \\
\ln (z)-\psi_{h}(z / L)=\frac{\kappa}{\theta_{*}} \theta-\frac{\kappa}{\theta_{*}} \theta_{0}+\ln \left(z_{0}\right)
\end{gathered}
$$




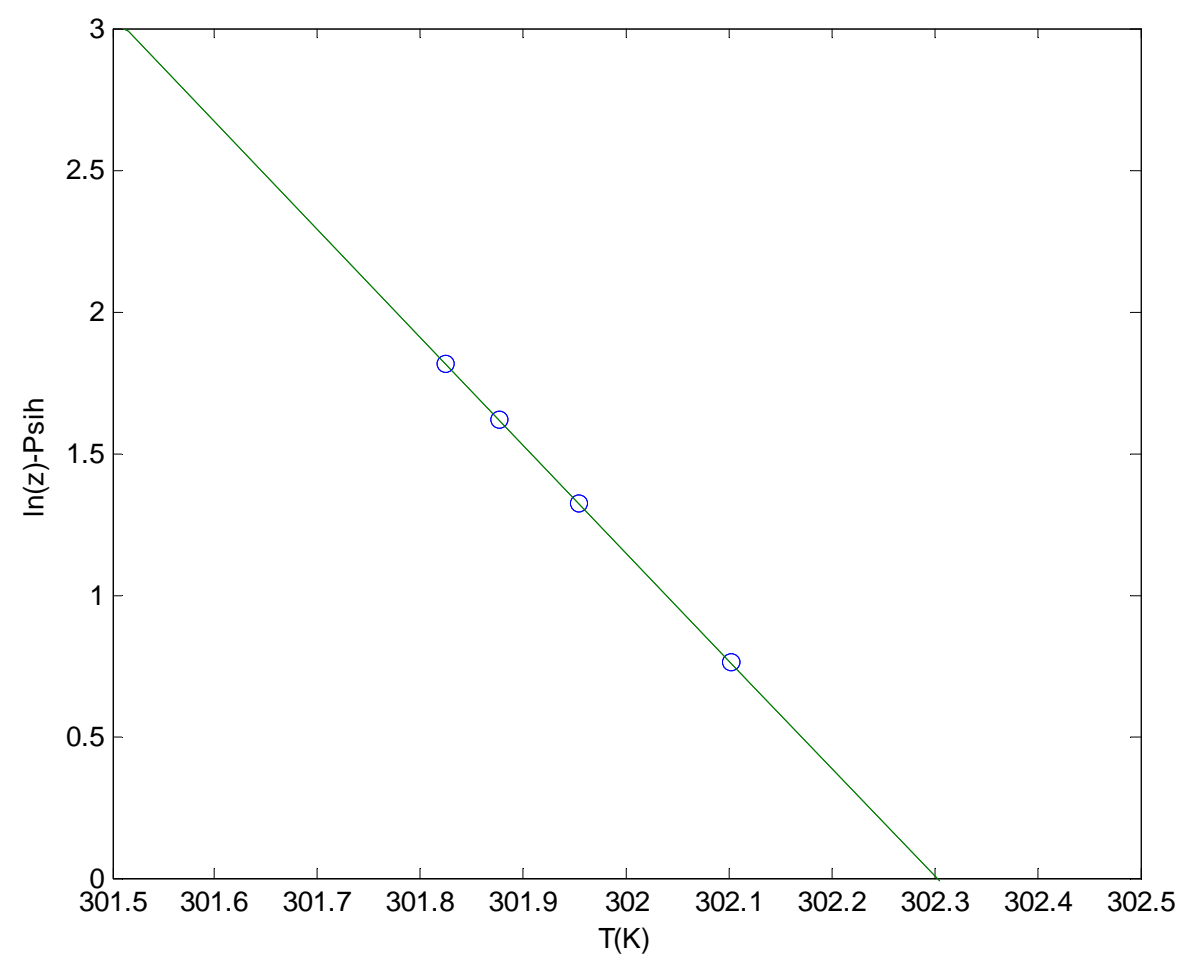

Figure B-3: Least square fitting of flux profile relation (modified log law) to observed unstable mean potential temperature profile

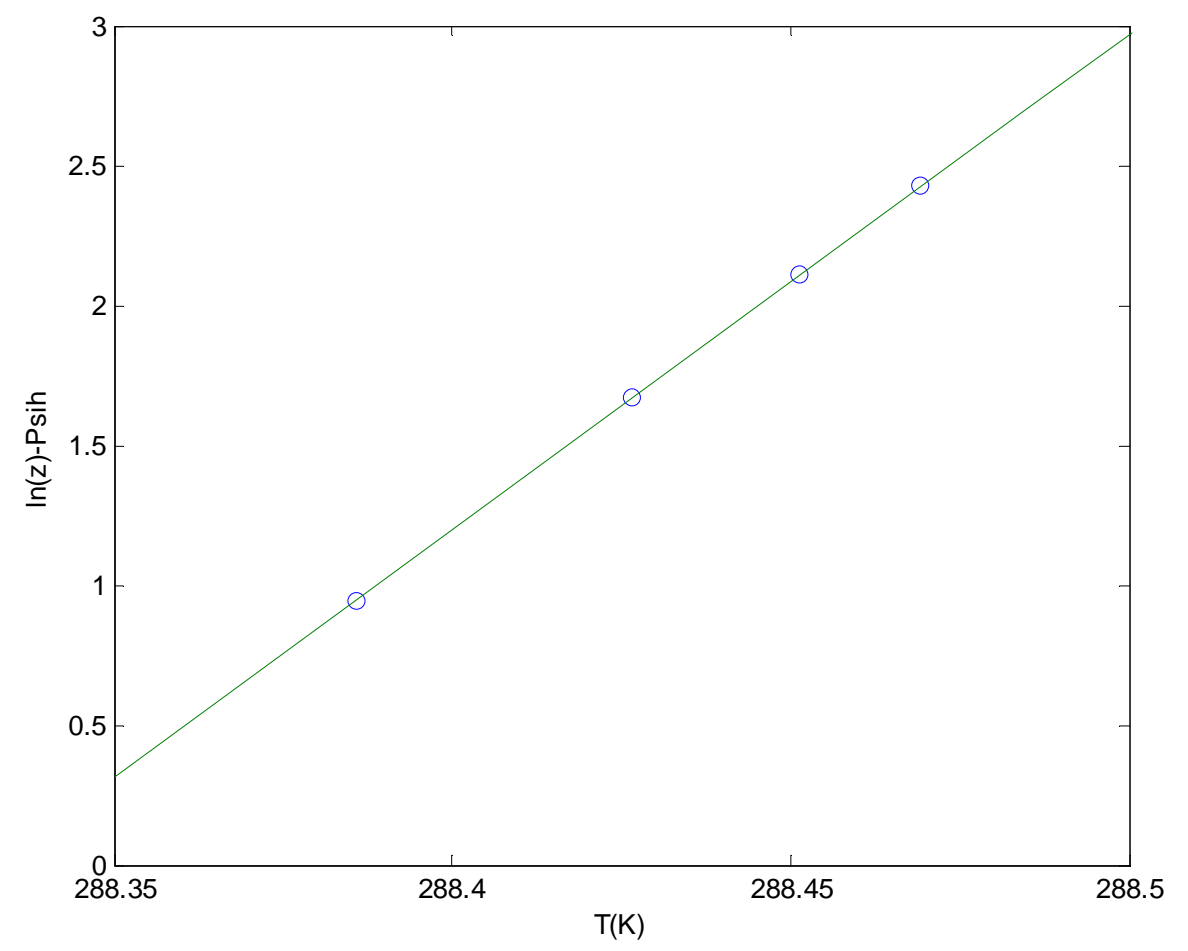

Figure B-4: Least square fitting of flux profile relation (modified log law) to observed stable mean potential temperature profile 


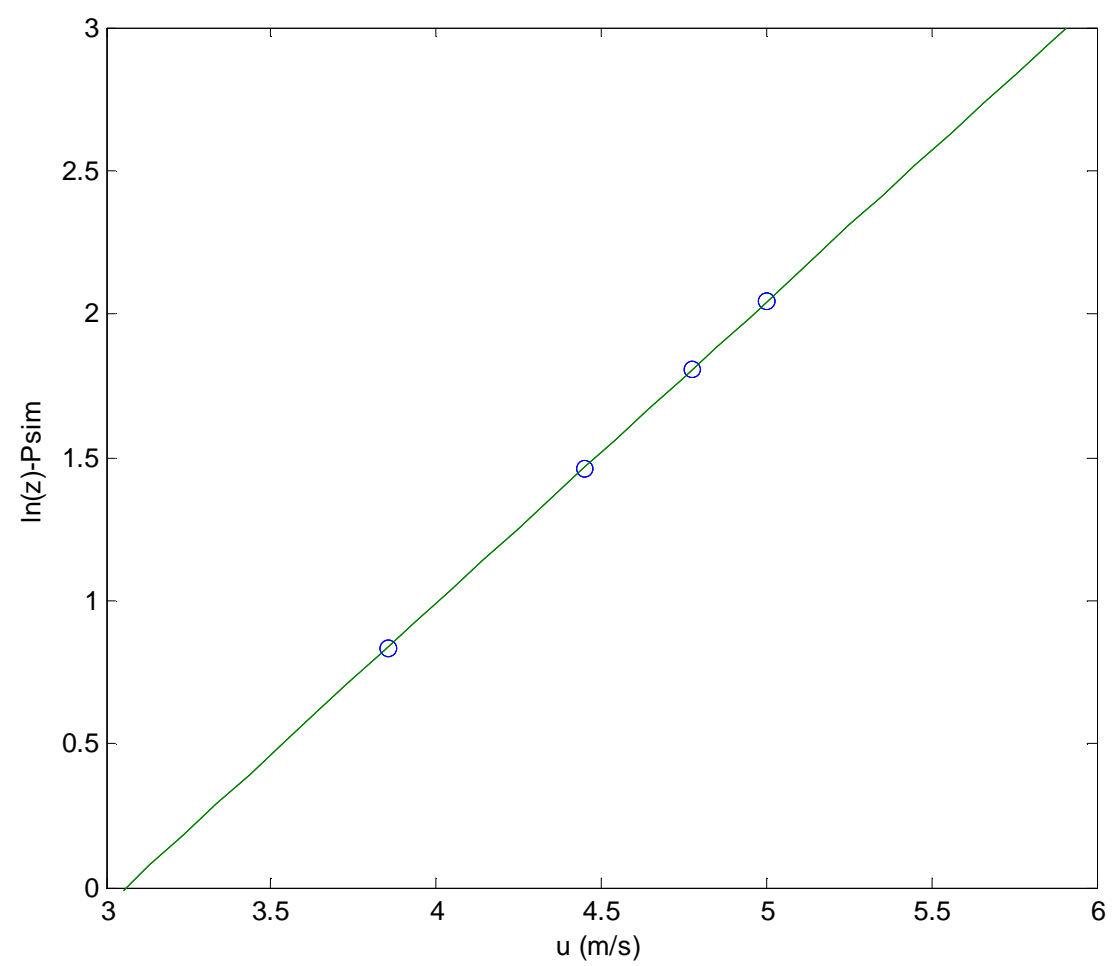

Figure B-5: Least square fitting of flux profile relation (modified log law) to observed unstable mean velocity profile

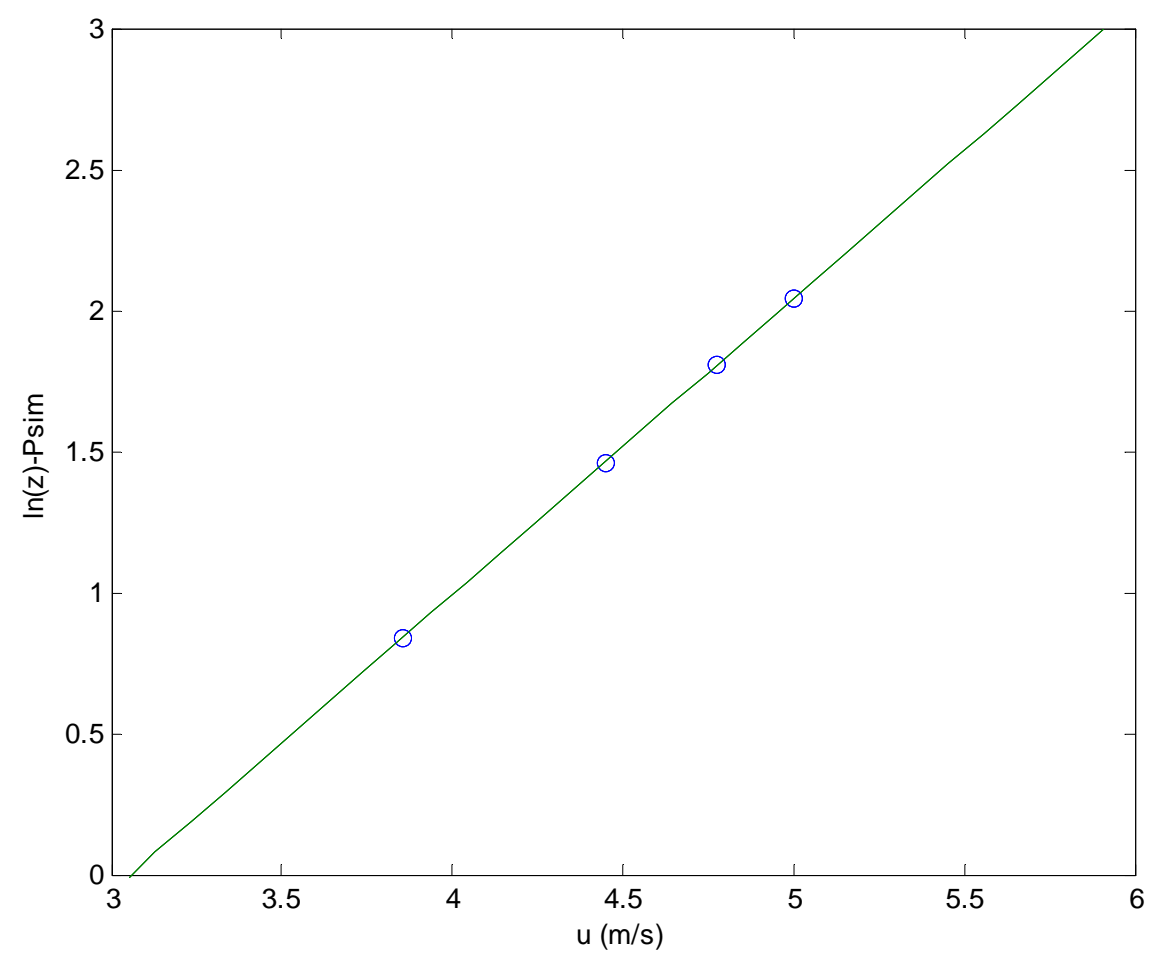

Figure B-6: Least square fitting of flux profile relation (modified log law) to observed stable mean velocity profile 
it can be seen that the intercepts of the plotted lines of $u$ versus $\ln (z)-\psi_{m}(z / L)$ and $\theta$ versus $\ln (z)-\psi_{h}(z / L)$ can be used to determine $z_{0}$ and $T_{0}$.

For the unstable case:

$$
\ln \left(z_{0}\right)=-3.2169
$$

Therefore:

$$
z_{0}=e^{-3.2169}=0.04 \mathrm{~m}
$$

And:

$$
-\frac{\kappa}{\theta_{*}} \theta_{0}+\ln \left(z_{0}\right)=1146.5
$$

Therefore:

$$
\begin{gathered}
-(-3.7924) \theta_{0}-3.2169=1146.5 \\
\theta_{0}=T_{0}=303.15 \mathrm{~K}
\end{gathered}
$$

And for the stable case:

$$
\ln \left(z_{0}\right)=-3.2186
$$

Therefore:

$$
z_{0}=e^{-3.2186}=0.04 \mathrm{~m}
$$

And:

$$
-\frac{\kappa}{\theta_{*}} \theta_{0}+\ln \left(z_{0}\right)=-5103.6
$$

Therefore:

$$
\begin{gathered}
-(17.701) \theta_{0}-3.2186=-5103.6 \\
\theta_{0}=T_{0}=288.15 \mathrm{~K}
\end{gathered}
$$

The surface fluxes are then given by:

$$
\begin{gathered}
\tau_{0}=\rho u_{*}^{2} \\
\dot{q}_{0}=-\rho c_{p} u_{*} \theta_{*}
\end{gathered}
$$


Where the density is calculated through the ideal gas law using the ground temperature and the reference (surface) pressure:

$$
\rho=\frac{P}{R T_{0}}
$$

For the unstable case:

$$
\begin{gathered}
\rho=\frac{101325}{(287.08)(303.15)}=1.1643 \mathrm{~kg} / \mathrm{m}^{3} \\
\tau_{0}=\rho u_{*}^{2}=(1.1643)(0.3897)^{2}=0.1768 \mathrm{~Pa} \\
\dot{q}_{0}=-\rho c_{p} u_{*} \theta_{*}=-(1.1643)(1003.62)(0.3897)(-0.1081)=49.24 \mathrm{~W} / \mathrm{m}^{2}
\end{gathered}
$$

And for the stable case:

$$
\begin{gathered}
\rho=\frac{101325}{(287.08)(288.15)}=1.2249 \mathrm{~kg} / \mathrm{m}^{3} \\
\tau_{0}=\rho u_{*}^{2}=(1.2249)(0.3632)^{2}=0.1616 \mathrm{~Pa} \\
\dot{q}_{0}=-\rho c_{p} u_{*} \theta_{*}=-(1.2249)(1003.62)(0.3632)(0.0232)=-10.34 \mathrm{~W} / \mathrm{m}^{2}
\end{gathered}
$$

The results of this analysis are summarised in Table B-4.

Table B-4: Results of analysis

\begin{tabular}{ccccccc}
\hline $\boldsymbol{L}$ & $\boldsymbol{u}_{*}$ & $\boldsymbol{T}_{*}$ & $\boldsymbol{z}_{\mathbf{0}}$ & $\boldsymbol{T}_{\mathbf{0}}$ & $\dot{\boldsymbol{q}}_{\mathbf{0}}$ & $\boldsymbol{\tau}_{\mathbf{0}}$ \\
\hline-105.30 & 0.390 & -0.108 & 0.040 & 30.00 & 49.24 & 0.177 \\
408.57 & 0.363 & 0.0232 & 0.040 & 15.00 & -10.34 & 0.162 \\
\hline
\end{tabular}




\section{APPENDIX C: NATURAL CONVECTION BUOYANCY DRIVEN FLOW}

The purpose of this exercise was to demonstrate the method of inducing buoyancy driven flow by density and temperature variation through the ideal gas law in a hydrostatic pressure field, brought about by adding the weight of the fluid to the momentum equation. The case of natural convection on an infinitely thin vertical heated plate is investigated. The flow is assumed to be laminar and the numerical results can be compared to analytical results. The computational domain is shown in Figure C-1.

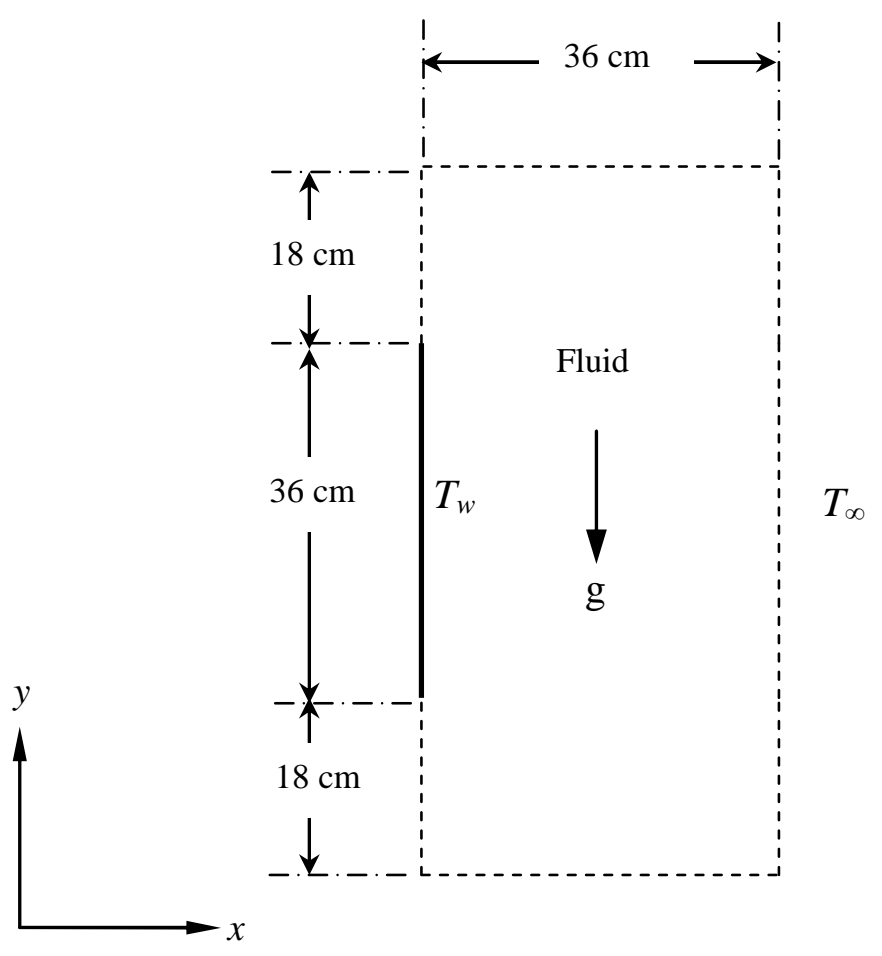

Figure C-1: Geometry of computational domain (Scale 1:10)

The geometry envisioned was a vertical plate immersed in air above the ground. The dimensions and fluid properties are summarised in Table C-1. The plate temperature $\left(T_{w}\right)$ is $294 \mathrm{~K}$ and it is surrounded by atmospheric air with temperature that follows the adiabatic lapse rate, with the ground temperature $\left(T_{o}\right)$ being $285 \mathrm{~K}$.

The computational mesh was created in GAMBIT, an easy way of generating a two-dimensional mesh, and is shown in Figure C-2. It consisted of 3321 vertices along 41 vertical lines, 20 horizontal lines below the plate, 20 horizontal lines above the plate and 41 horizontal lines perpendicular to the plate. The horizontal lines near the plate ends were spaced closer together with a successive ratio of 1.08 , with spacing increasing further form the plate ends on either side. The resulting two-dimensional mesh consisted of 3200 cells. The top and right fluid boundaries were specified as a pressure outlet and the bottom fluid boundary was 
a wall. The left vertical fluid boundaries above and below the plate (also specified as a wall) ends were specified as symmetry boundaries.

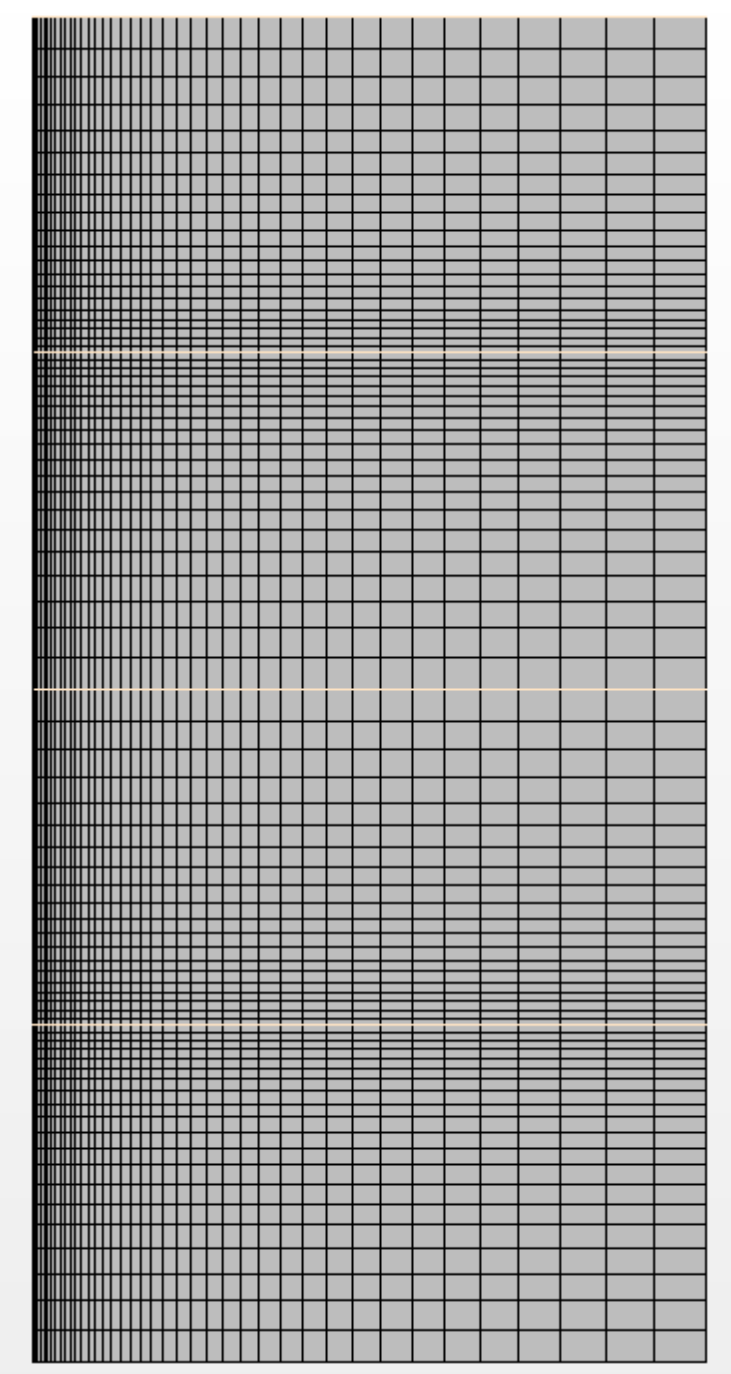

Figure C-2: Computational grid

The simulation space was specified as two dimensional and the steady flow equations were solved. The fluid was selected as air with the density calculated through the ideal gas law. The segregated flow solver was chosen, which achieves pressure-velocity coupling through the SIMPLE algorithm (CD-adapco Inc. 2011), and solves the total energy equation. Convection of momentum and energy was solved using the second-order upwind scheme. The laminar viscous regime and a reference pressure of $101325 \mathrm{~Pa}$ were used.

The ground boundary had the no-slip shear stress specification selected and thermal specification was achieved through a static temperature of $285 \mathrm{~K}$, which would serve as the ground temperature. Similarly the vertical plate also had had 
the no-slip shear stress specification selected and thermal specification was achieved through a static temperature of $294 \mathrm{~K}$. Both of these wall boundaries were assumed to be smooth.

The top and side pressure outlet boundaries had "backflow direction specification" set to "extrapolated". At the side boundary the pressure was specified using the user defined "field function" for the pressure of the neutral atmosphere as given by Equation 2-10, relative to the reference pressure and using the temperature of the ground. Additionally, the temperature at this boundary was specified using the user defined "field function" of the adiabatic lapse rate temperature as found in the neutral atmosphere, described by Equation 2-7, relative to the ground temperature. The pressure outlet boundary at the top of the domain had the pressure and temperature predicted from Equations 2-10 and 2-7, respectively, at the height of the domain specified as constant values.

Additionally a source term was added to the momentum equation using the "momentum source option" to account for the weight of the fluid and thereby add the effect of gravity. Input was achieved using a user defined vector "field function" in the form:

$$
S_{m}=\rho g_{i}
$$

where the gravitational acceleration is also a user defined vector "field function". The under-relaxation factors employed by the solver are given in Table C-2.

Table C-1: Properties used in calculation

\begin{tabular}{lc}
\multicolumn{1}{c}{ Parameters } & Value \\
\hline Plate Length; $L_{w}[\mathrm{~m}]$ & 0.36 \\
Plate temperature; $T_{w}[\mathrm{~K}]$ & 294 \\
Ground temperature; $T_{o}[\mathrm{~K}]$ & 285 \\
Gravitational acceleration; $g\left[\mathrm{~m} / \mathrm{s}^{2}\right]$ & 9.81 \\
Air heat capacity; $c_{p}[\mathrm{~J} / \mathrm{kg} \cdot \mathrm{K}]$ & 1003.62 \\
Air conductivity; $\lambda[\mathrm{W} / \mathrm{m} \cdot \mathrm{K}]$ & 0.0260305 \\
Fluid dynamic viscosity; $\mu[\mathrm{kg} / \mathrm{ms}]$ & $1.86 \mathrm{E}-05$ \\
\hline
\end{tabular}

The simulation was initialised with the pressure values of the "field function" for the neutral atmosphere, the temperature from the "field function" defining the adiabatic lapse rate and zero velocity. The solution was run until the residuals converged. 
Table C-2: Under-relaxation factors for flow simulation

\begin{tabular}{ll}
\hline Pressure & 0.3 \\
Velocity & 0.7 \\
Energy & 0.9 \\
\hline
\end{tabular}

Analytical boundary layer solutions to the wall shear stress and heat flux for steady state laminar natural convection on a vertical isothermal plate are available (Mills 1999). The solution is expressed in terms of two dimensionless numbers, the Prandtl $(P r)$ number and the Grashof $(G r)$ number:

$$
\begin{gathered}
\operatorname{Pr}=\frac{c_{p} \mu}{\lambda} \\
G r=\frac{\rho^{2} g \beta\left(T_{w}-T_{\infty}\right) L_{w}^{3}}{\mu^{2}}
\end{gathered}
$$

where $\alpha$ is the thermal conductivity, and the thermal expansion coefficient can be calculated for an ideal gas as the inverse of the temperature. In the solution that follows, $y$ denotes the distance along the plate measured from the leading edge. It must be noted that the underlying assumption of this solution is that the boundary layer starts at the leading edge and that the plate is infinitely long. The analytical solution therefore does not account for "edge effects". The local wall shear stress can be calculated as:

$$
\begin{gathered}
\tau_{w}=\frac{\mu^{3}}{\rho} f(P r)\left(\frac{4 G r^{3} y}{L_{w}^{9}}\right)^{1 / 4} \\
f(\operatorname{Pr}) \approx \log _{10}\left(4.4 \operatorname{Pr}^{-\frac{1}{5}}\right) \quad \text { for } 0.5 \leq \operatorname{Pr} \leq 2
\end{gathered}
$$

while the mean shear stress can be calculated by:

$$
\overline{\tau_{w}}=\frac{4 \mu^{2}}{5 \rho L_{w}^{2}} f(P r)\left(4 G r^{3}\right)^{\frac{1}{4}}
$$

Furthermore the local wall heat flux is obtained by: 


$$
\begin{gathered}
\dot{q}_{w}=\lambda\left(T_{w}-T_{\infty}\right) g(\operatorname{Pr})\left(\frac{G r}{4 y L_{w}^{3}}\right)^{\frac{1}{4}} \\
g(\operatorname{Pr}) \approx \frac{0.676 \operatorname{Pr}^{\frac{1}{2}}}{(0.861+P r)^{\frac{1}{4}}} \quad \text { for } 0.01 \leq \operatorname{Pr} \leq 1000
\end{gathered}
$$

while the mean heat flux is:

$$
\dot{\overline{q_{w}}}=\frac{4 \alpha\left(T_{w}-T_{\infty}\right)}{3 L_{w}} g(\operatorname{Pr})\left(\frac{G r}{4}\right)^{\frac{1}{4}}
$$

In the above solutions $T_{\infty}$ is taken as $T_{o}$ and the expansion coefficient can be calculated using $T_{w}$. Using the values specified for this analysis the Prandtl and Grashof numbers could be calculated and are given in Table C-3.

Table C-3: Calculated Prandtl and Grashof numbers

Prandtl Number: $\mathrm{Pr}$

0.715236123

Grashof Number: $G r$

58678511.85

The numerical and analytical solutions could now be compared with the results for the wall shear stress shown in Figure C-3 and for the wall heat flux in Figure C-4. There is good correlation between the numerical and analytical results, with the discrepancies coming in at the edges due to the fact that the analytical solution assumes an infinitely long plate. The mean steady state shear stress and heat flux for the numerical and analytical solutions is summarised in Table C-4. We find generally good agreement between the two methods.

Table C-4: Mean steady state shear stress and heat flux

\begin{tabular}{llll}
\hline & Analytical & Numerical & Difference \\
\hline $\boldsymbol{\tau}_{\boldsymbol{w}}\left[\mathrm{N} / \mathrm{m}^{2}\right]$ & 0.00113 & 0.00112 & $0.68 \%$ \\
$\boldsymbol{q}_{\boldsymbol{w}}\left[\mathrm{W} / \mathrm{m}^{2}\right]$ & 27.4 & 29.6 & $8.02 \%$ \\
\hline
\end{tabular}




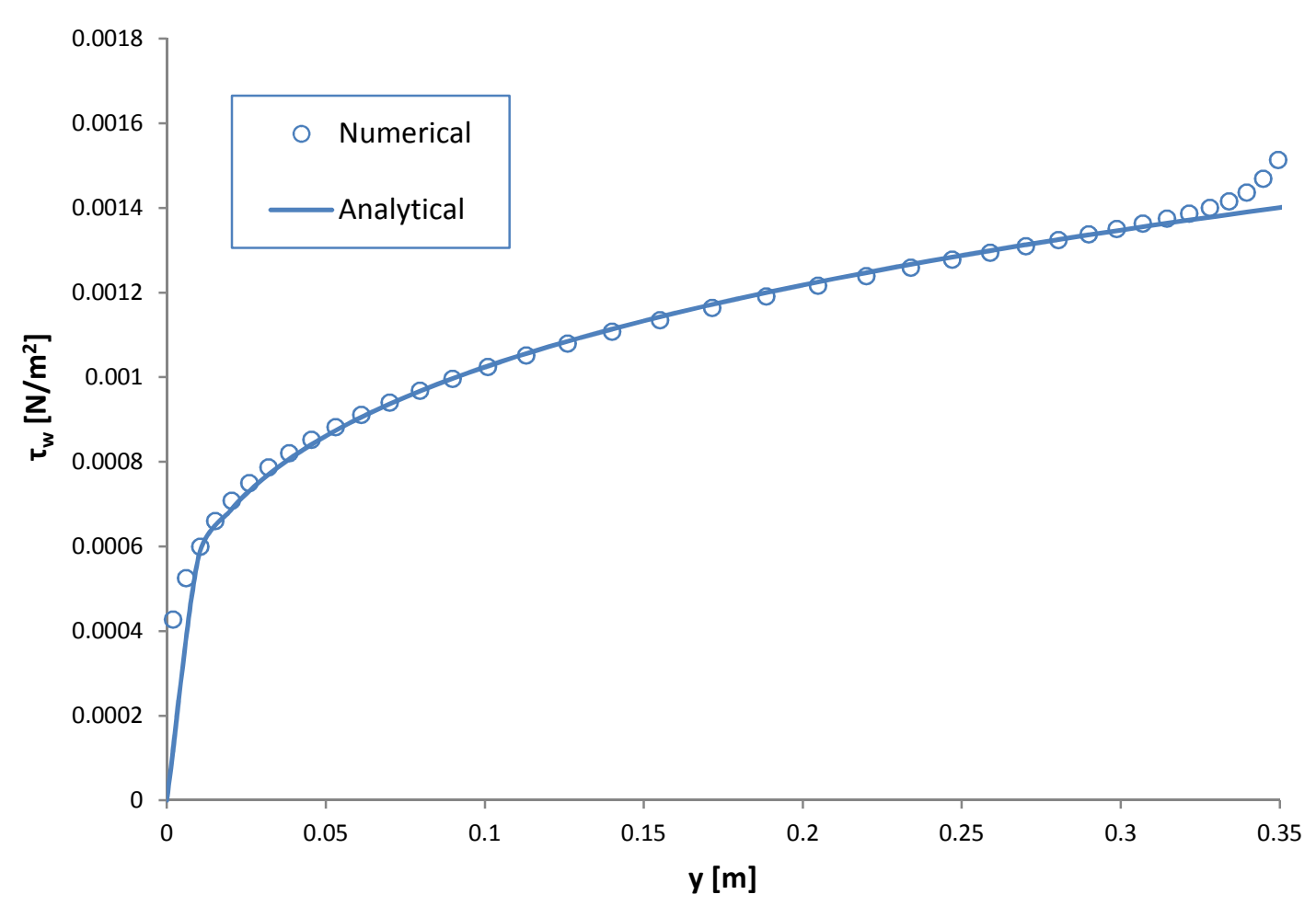

Figure C-3: Steady state wall shear stress on vertical plate

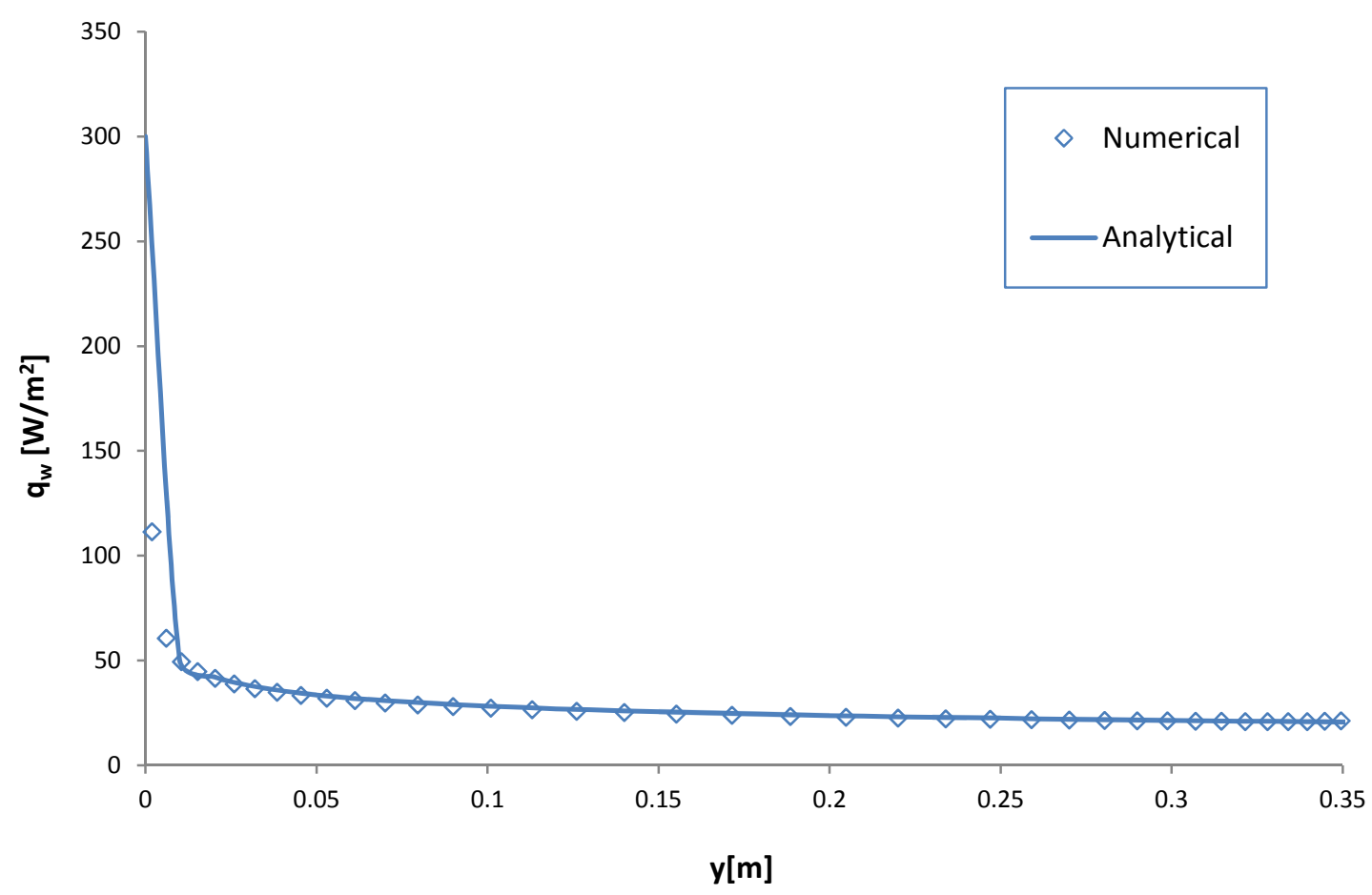

Figure C-4: Steady state wall heat flux on vertical plate 
The development of the steady state boundary layer velocity profile is shown in Figure C-5. The dimension $y$ is measured from the bottom edge of the plate. The result shows an increase in the peak velocity as one moves further up from the bottom of the plate, and a distinctly upward flow. For the three values of $y$ equal to 0 (bottom end of the plate), $L / 2$ (half of the plate length) and $L$ we observe the no-slip condition, where the points close to the wall display nearly zero velocity. For the profile along the line at $y$ equal to $3 L / 2$ we have moved past the plate into the free stream and therefore see no zero velocity where $x$ is close to zero, but rather the peak velocity value. The velocity steadily decreases in the positive $x$ direction and becomes negative as air is drawn in from the surroundings due to the convective motion.

The steady state temperature boundary layer results are given in Figure C-6. It is observed that for $y$ equal to $0, L / 2$ and $L$ the temperature difference is virtually equal to the difference between $T_{w}$ and $T_{o}$ as given in Table $\mathrm{C}-1$. These points are very close to the wall and therefore close to the wall temperature. It is clear that as one moves further up the plate the temperature boundary layer becomes thicker than at the lower edge, but the temperature does not penetrate very far into the flow. It is also seen that for y equal to $3 L / 2$, the temperature starts to dissipate and it has a peak temperature lower than the plate temperature.

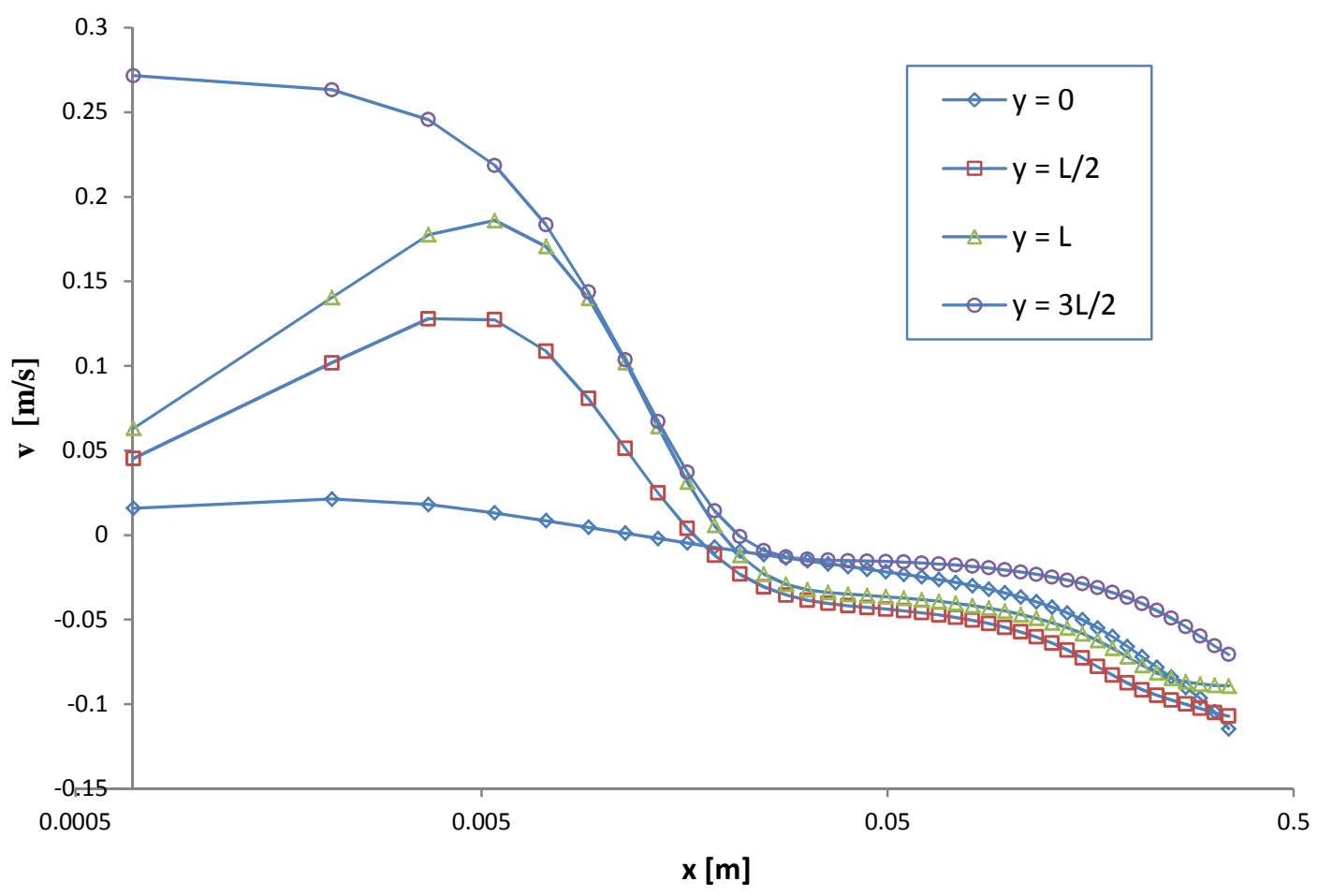

Figure C-5: Development of steady state vertical velocity boundary layer 


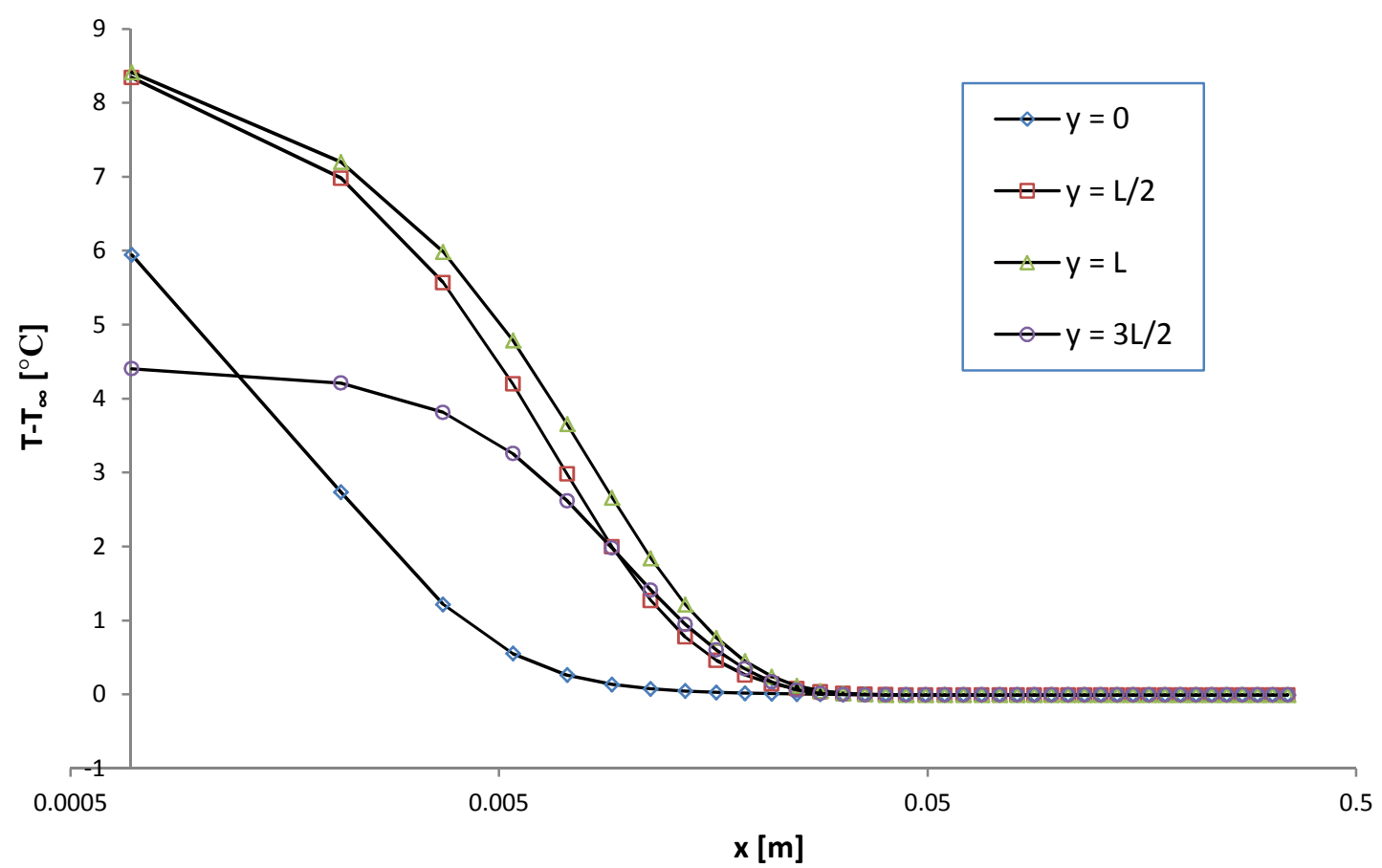

Figure C-6: Development of steady state temperature boundary layer

From the results it is clear that the method of adding the gravity term to the momentum equation and using the ideal gas law, the temperature differences specified in this example lead to density differences which lead to the development of a buoyancy force. The buoyancy force thus developed leads to the expected result of upward vertical flow and the convection of heat upwards in the fluid domain, as was observed with the development of the temperature boundary layer. This upward flow develops a shear stress and heat flux on the surface of the vertical plate that corresponds well with the analytical solution for the same case. The results are consistent with what is expected for natural convection buoyancy driven flow.

The temperature and pressure predicted for the neutral atmosphere was applied for consistency with the full scale ABL flow CFD models, even though the change with height over the scale represented by the flow domain in this analysis will be almost negligible. Furthermore a pressure had to be specified at the pressure outlet that was consistent with the weight of the fluid and the resultant changes in density and temperature.

It can be concluded that buoyancy is sufficiently accounted for by using this technique. It should be noted though that numerical instability is likely to emerge for larger scale low-velocity applications, where the pressure is more difficult to control in the absence of flow, making it more difficult to nail down the density. Other methods of buoyancy handling, like the Boussinesq approximation, are therefore likely to be numerically more stable. 


\section{APPENDIX D: GRID SENSITIVITY ANALYSIS FOR COSINE HILL SIMULATION}

The solution errors from discretization were estimated using Richardson extrapolation and the Grid Convergence Index Method (GCI), proposed by Celik et al. (2008). The domain containing the cosine hill was meshed using the meshing models described in Chapter 6 . It can be seen that the grid is generated by defining a base size. In the case of the grid used in Chapter 6 the base size was $40 \mathrm{~mm}$, and it consisted of 602796 cells. Two other grids were generated by changing the base size. A coarser grid, with a base size of $50 \mathrm{~mm}$, was generated and consisted of 328031 cells. A finer grid, with a base size of $30 \mathrm{~mm}$, was also generated and this consisted of 1363693 cells.

The first step in the procedure for the estimation of discretisation error is to define a representative cell, mesh, or grid size $j$. For three dimensional calculations:

$$
j=\left[\frac{1}{N} \sum_{i=1}^{N}\left(\Delta V_{i}\right)\right]^{1 / 3}
$$

where $\Delta V_{i}$ is the volume of the $i$ th cell and $N$ is the total number of cells. The representative cell size was found to be $0.0386 \mathrm{~m}$ for the coarse grid, $0.0297 \mathrm{~m}$ for the medium grid and $0.0226 \mathrm{~m}$ for the fine grid. The next step is to define a grid refinement factor $f$ :

$$
f=\frac{j_{\text {coarse }}}{j_{\text {fine }}}
$$

This must be greater than 1.3 for each consecutive refinement. It is important that the refinement be structured, as is achieved by changing the base value as described above. The simulation results were calculated using the SST $k-\omega$ turbulence model on each grid and the values of key variables $(\alpha)$ that are considered important in the investigations were defined. For the cosine hill simulation the re-attachment length, the horizontal streamwise velocity and turbulent kinetic energy at the position $x / H$ of 2.25 and $z / H$ of 0.5 were used as the key variables, where $H$ is the height of the hill. These variables are dependent on the accurate simulation of the separation bubble in the wake of the hill and the largest discretisation errors were observed in this region.

Now if $j_{1}<j_{2}<j_{3}$ and:

$$
f_{21}=\frac{h_{2}}{h_{1}}
$$




$$
f_{32}=\frac{h_{3}}{h_{2}}
$$

The apparent order $p$ of the method can be calculated by the expression:

$$
\begin{gathered}
p=\frac{1}{\ln \left(r_{21}\right)}|\ln | \sigma_{32} / \sigma_{21}|+q(p)| \\
q(p)=\ln \left(\frac{f_{21}^{p}-s}{f_{32}^{p}-s}\right) \\
s=1 \cdot \operatorname{sgn}\left(\sigma_{32} / \sigma_{21}\right) \\
\sigma_{32}=\alpha_{3}-\alpha_{2} \\
\sigma_{21}=\alpha_{2}-\alpha_{1}
\end{gathered}
$$

where $\alpha_{n}$ denotes the solution of the $n$th grid. Equation D-5 can be solved using fixed point iteration with the initial guess equal to the first term. The extrapolated values can now be calculated from:

$$
\alpha_{\text {ext }}^{21}=\left(f_{21}^{p} \alpha_{1}-\alpha_{2}\right) /\left(f_{21}^{p}-1\right)
$$

and $\alpha_{\text {ext }}^{32}$ can be similarly calculated. The different errors can now be calculated and reported. The approximate relative error is defined as:

$$
e_{a}^{21}=\left|\frac{\alpha_{1}-\alpha_{2}}{\alpha_{1}}\right|
$$

The extrapolated relative error is:

$$
e_{\text {ext }}^{21}=\left|\frac{\alpha_{\text {ext }}^{21}-\alpha_{1}}{\alpha_{\text {ext }}^{21}}\right|
$$

And the fine grid convergence index is:

$$
\mathrm{GCI}_{\text {fine }}^{21}=\frac{1.25 e_{a}^{21}}{f_{21}^{p}-1}
$$


The results of the above procedure are reported in Table D-1. It can be seen that the grid refinement factors are sufficiently large. Furthermore, the relative error between the medium and the fine grid, the extrapolated error for fine grid, and the grid convergence index, and thus the numerical uncertainty in the fine grid solution, for all three of the key variables is found to be less than $1 \%$. It was therefore decided to use the medium grid for all the subsequent simulations, as the discretisation error made using this grid was not much greater than for the fine grid, which came very close to the extrapolated solution values. The medium grid also did not require the large computing requirements associated with the fine grid.

Table D-1: Results of discretisation error calculation for cosine hill simulation

\begin{tabular}{|c|c|c|c|}
\hline & $\begin{array}{c}\alpha=\text { Re-attachment } \\
\text { Length } \\
{[\mathrm{m}]}\end{array}$ & $\begin{array}{c}\alpha=\text { Horizontal } \\
\text { Velocity at } x / H=2.25 \\
z / H=0.5 \\
{[\mathrm{~m} / \mathrm{s}]}\end{array}$ & $\begin{array}{c}\alpha=\text { Turbulent Energy } \\
\text { at } x / H=2.25, z / H=0.5 \\
{\left[\mathrm{~m}^{2} / \mathrm{s}^{2}\right]}\end{array}$ \\
\hline$N_{1}, N_{2}, N_{3}$ & $\begin{array}{c}\text { 1363693, 602796 } \\
328031\end{array}$ & $\begin{array}{c}\text { 1363693, 602796 } \\
328031\end{array}$ & $\begin{array}{c}\text { 1363693, 602796, } \\
328031\end{array}$ \\
\hline$f_{21}$ & 1.31 & 1.31 & 1.31 \\
\hline$f_{32}$ & 1.30 & 1.30 & 1.30 \\
\hline$\alpha_{1}$ & 2.262 & 0.571 & 0.0725 \\
\hline$\alpha_{2}$ & 2.259 & 0.576 & 0.0730 \\
\hline$\alpha_{3}$ & 2.264 & 0.523 & 0.0739 \\
\hline$p_{21}$ & 1.77 & 10.91 & 2.87 \\
\hline$\alpha_{e x t}^{21}$ & 2.268 & 0.570 & 0.0720 \\
\hline$e_{a}^{21}$ & $0.15 \%$ & $0.96 \%$ & $0.78 \%$ \\
\hline$e_{e x t}^{21}$ & $0.25 \%$ & $0.05 \%$ & $0.66 \%$ \\
\hline $\mathbf{G C I}_{\text {fine }}^{21}$ & $0.31 \%$ & $0.06 \%$ & $0.82 \%$ \\
\hline
\end{tabular}




\section{APPENDIX E: GRID SENSITIVITY ANALYSIS FOR TRANSVERSE DUNE SIMULATION}

The solution errors from discretization were estimated using Richardson extrapolation and the Grid Convergence Index Method (GCI), proposed by Celik et al. (2008), as explained in Appendix D. The domain containing the dune was meshed using the meshing models described in Chapter 7. Here too the base size was changed to generate a coarser and a finer grid in a structured manner. The medium grid had a base size of $5 \mathrm{~m}$, and it consisted of 17328 cells. The coarser grid, with a base size of $10 \mathrm{~m}$, consisted of 6717 cells. The finer grid, with a base size of $3 \mathrm{~m}$, consisted of 38755 cells. All the grids were two dimensional.

The representative cell, mesh, or grid size $j$ was therefore calculated for two dimensions by:

$$
j=\left[\frac{1}{N} \sum_{i=1}^{N}\left(\Delta A_{i}\right)\right]^{1 / 2}
$$

where $\Delta A_{i}$ is the area of the $i$ th cell and $N$ is the total number of cells. The representative cell size was found to be $4.0213 \mathrm{~m}$ for the coarse grid, $3.0113 \mathrm{~m}$ for the medium grid and $2.01366 \mathrm{~m}$ for the fine grid.

The simulations were run on each grid using the SST $k$ - $\omega$ turbulence model under neutral atmospheric conditions. The key variables chosen were the re-attachment length, the horizontal streamwise velocity and turbulent kinetic energy at the position $x / H$ of 10 and $z / H$ of 0.5 , where $\mathrm{H}$ is the height of the dune. These variables were dependent on the accurate simulation of the wake region of the dune, where the largest discretization errors were also recorded. The same procedure was followed as explained in Appendix D, with the results of the discretisation error calculation given in Table E-1.

It can be seen that the grid refinement factors are sufficiently large. Furthermore, the relative error between the medium and the fine grid was found to have a maximum value of $5.3 \%$ (for the turbulent kinetic energy). The extrapolated error for fine grid was less than $2 \%$ in all cases while the grid convergence index, and thus the numerical uncertainty in the fine grid solution, had a maximum value of $2.35 \%$ (also for the turbulent kinetic energy). The Fine grid therefore had a small discretisation error associated with it, and the error between the medium and the fine grid was also small. It was therefore decided to use the medium grid for all the subsequent simulations, without the significant increase in computing requirements associated with the fine grid. 
Table E-1: Results of discretisation error calculation for dune simulation

\begin{tabular}{|c|c|c|c|}
\hline & $\begin{array}{c}\alpha=\text { Re-attachment } \\
\text { Length } \\
{[\mathrm{m}]}\end{array}$ & $\begin{array}{c}\alpha=\text { Axial Velocity at } \\
\mathbf{x} / \mathbf{H}=\mathbf{1 0}, \mathrm{z} / \mathrm{H}=\mathbf{0 . 5} \\
{[\mathrm{m} / \mathrm{s}]}\end{array}$ & $\begin{array}{c}\alpha=\text { Turbulent Energy } \\
\text { at } \mathbf{x} / \mathbf{H}=\mathbf{1 0}, \mathrm{z} / \mathrm{H}=\mathbf{0 . 5} \\
{\left[\mathrm{m}^{2} / \mathrm{s}^{2}\right]}\end{array}$ \\
\hline$N_{1}, N_{2}, N_{3}$ & $38755,17328,6717$ & $38755,17328,6717$ & $38755,17328,6717$ \\
\hline$f_{21}$ & 1.50 & 1.50 & 1.50 \\
\hline$f_{32}$ & 1.34 & 1.34 & 1.34 \\
\hline$\alpha_{1}$ & 232.875 & 2.861 & 2.2565 \\
\hline$\alpha_{2}$ & 230.625 & 3.000 & 2.1371 \\
\hline$\alpha_{3}$ & 223.750 & 3.311 & 1.8752 \\
\hline$p_{21}$ & 4.36 & 3.40 & 3.33 \\
\hline$\alpha_{e x t}^{21}$ & 233.346 & 2.813 & 2.2990 \\
\hline$e_{a}^{21}$ & $0.97 \%$ & $4.86 \%$ & $5.30 \%$ \\
\hline$e_{e x t}^{21}$ & $0.20 \%$ & $1.69 \%$ & $1.85 \%$ \\
\hline $\mathbf{G C I}_{\text {fine }}^{21}$ & $0.25 \%$ & $2.08 \%$ & $2.35 \%$ \\
\hline
\end{tabular}




\section{APPENDIX F: FURTHER SST K- $\Omega$ RESULTS FOR CFD SIMULATION OF THE ABL UNDER DIFFERENT THERMAL STRATIFICATIONS}

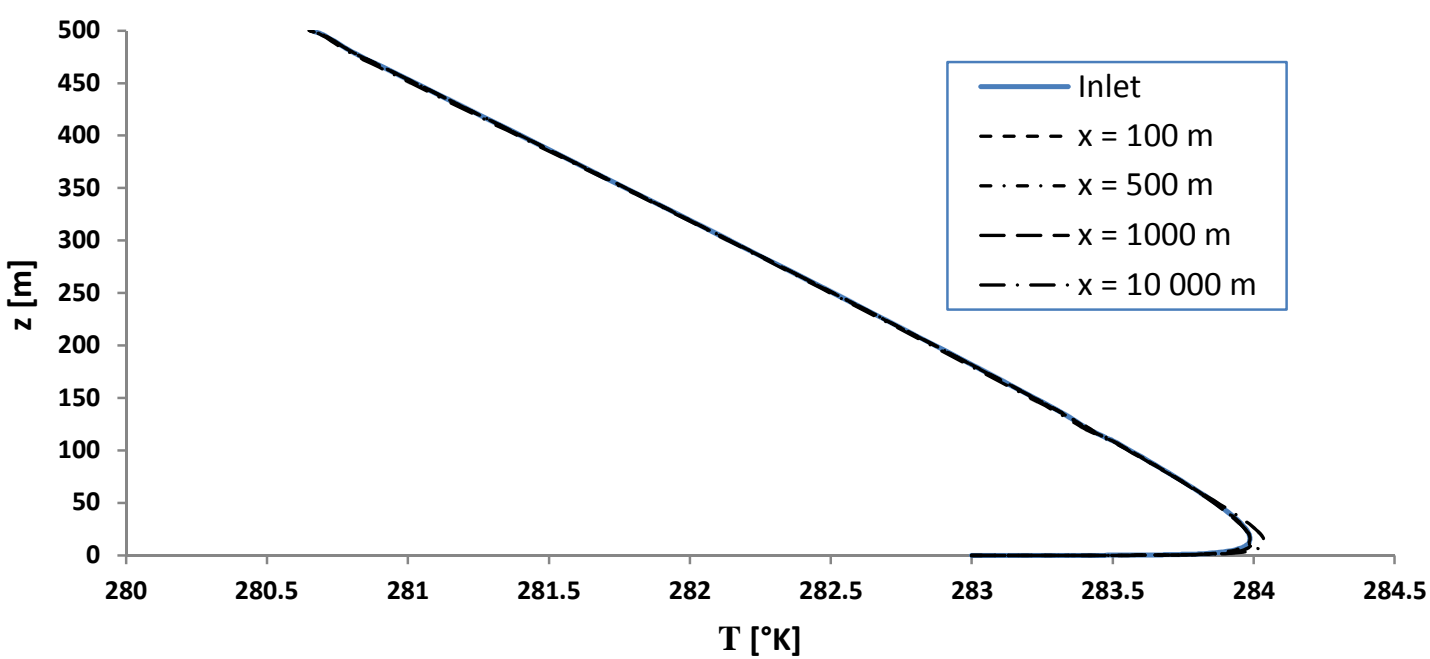

(a)

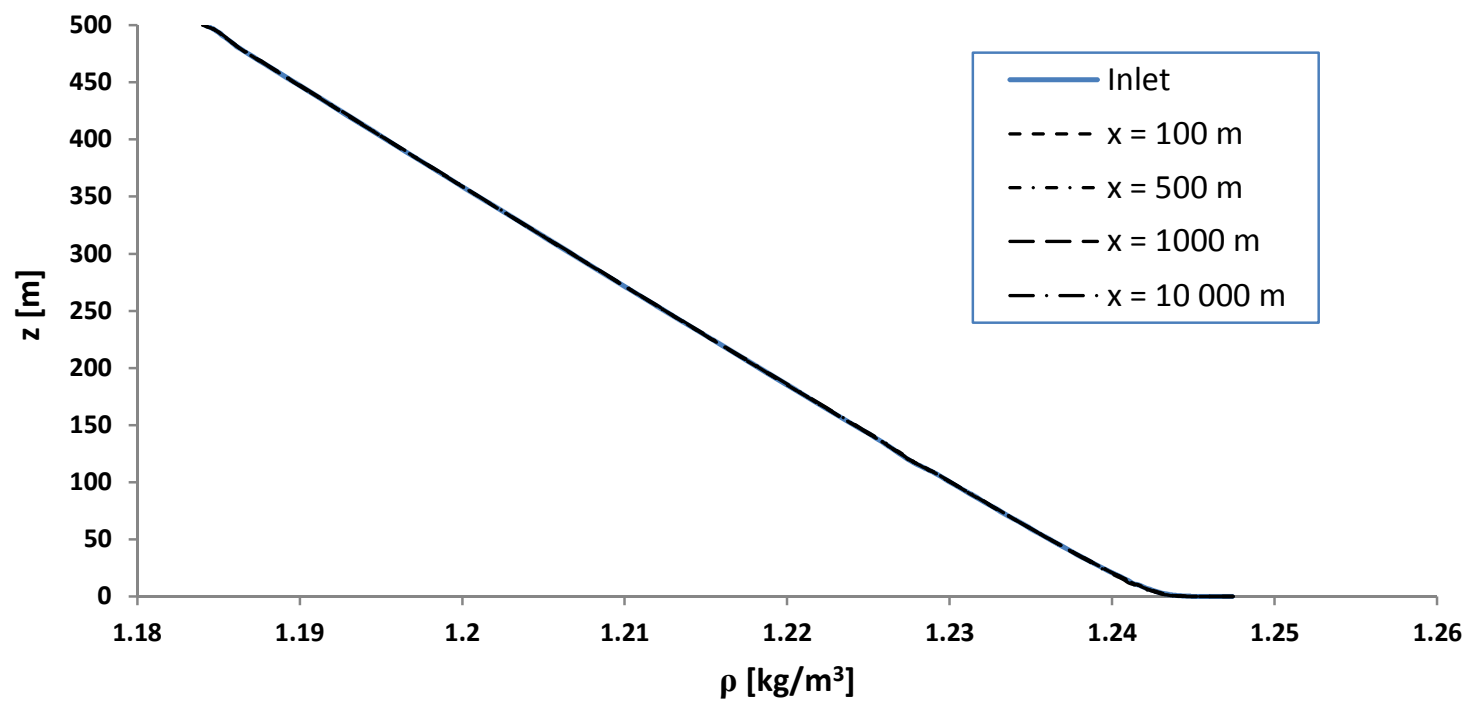

(b)

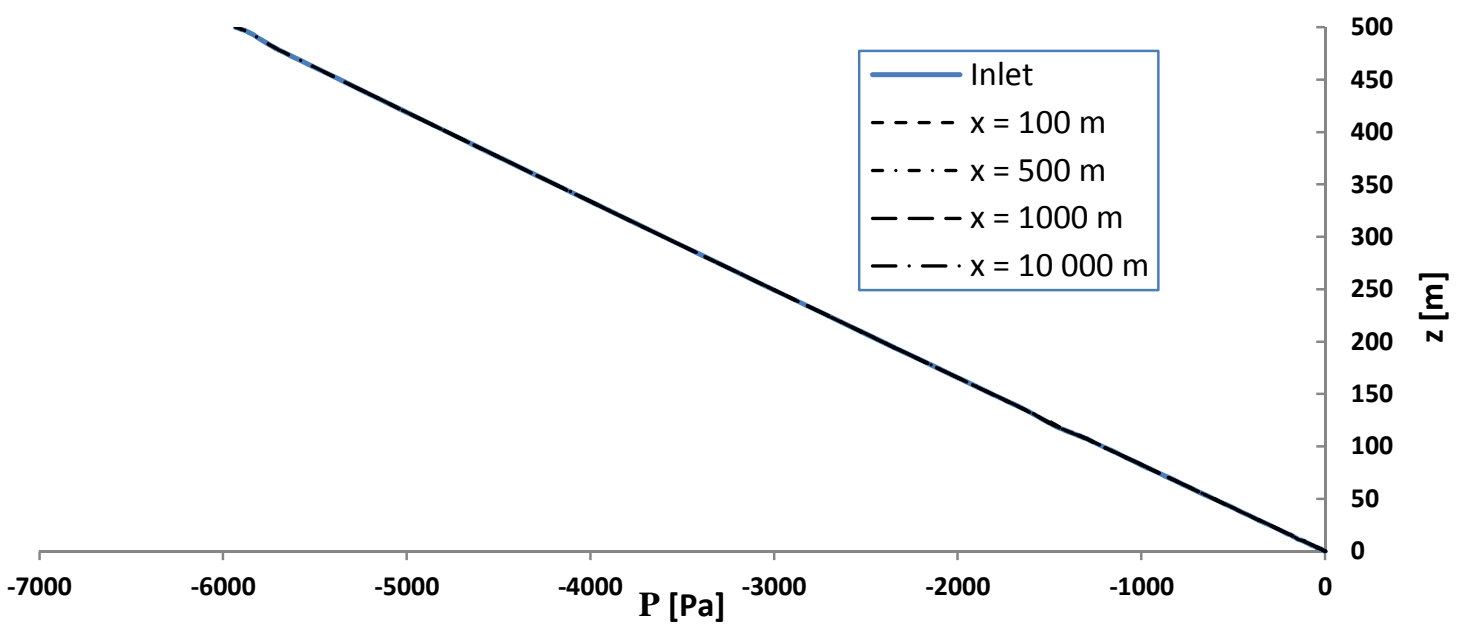

Figure F-1: Stable CFD $k-\omega$ model results illustrating streamwise gradients for (a) temperature $T$; (b) density $\rho$; and (c) pressure $P$. 

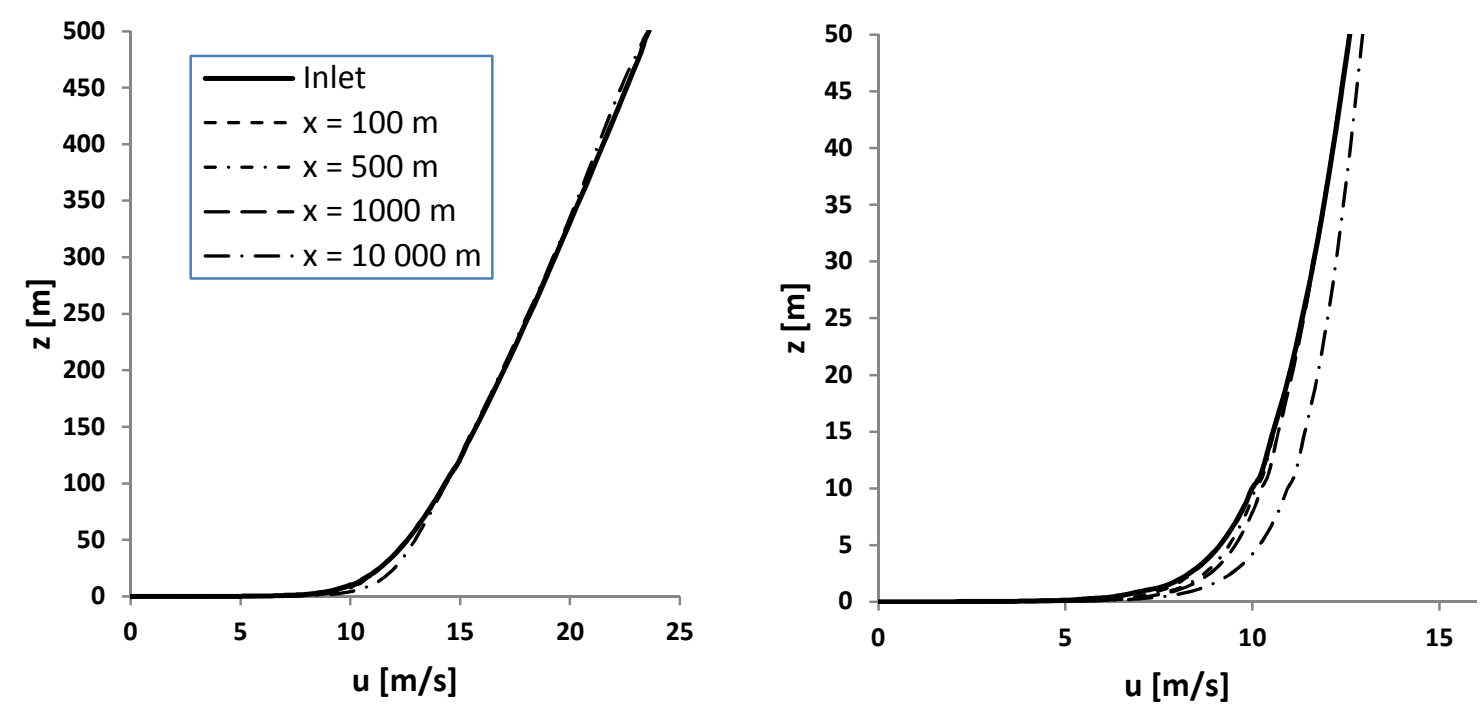

(a)
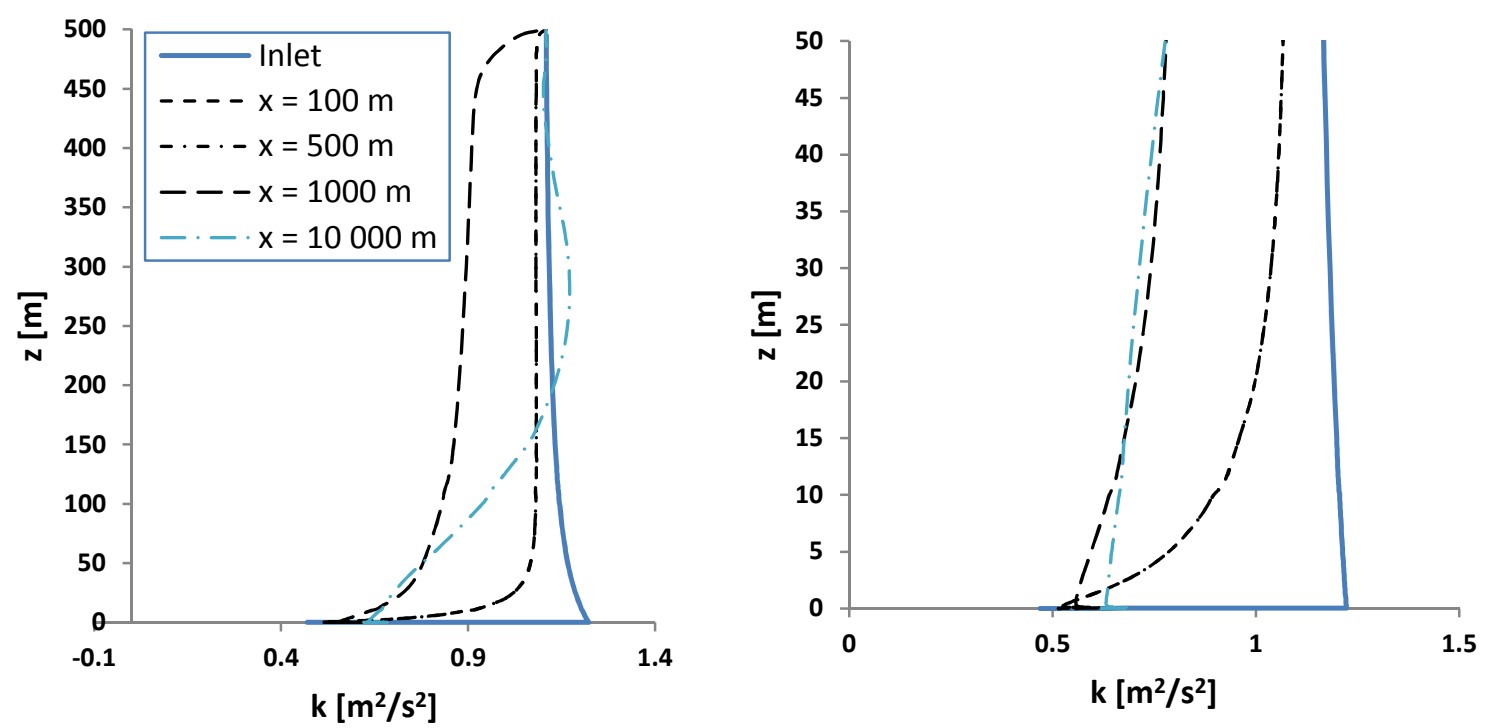

(b)

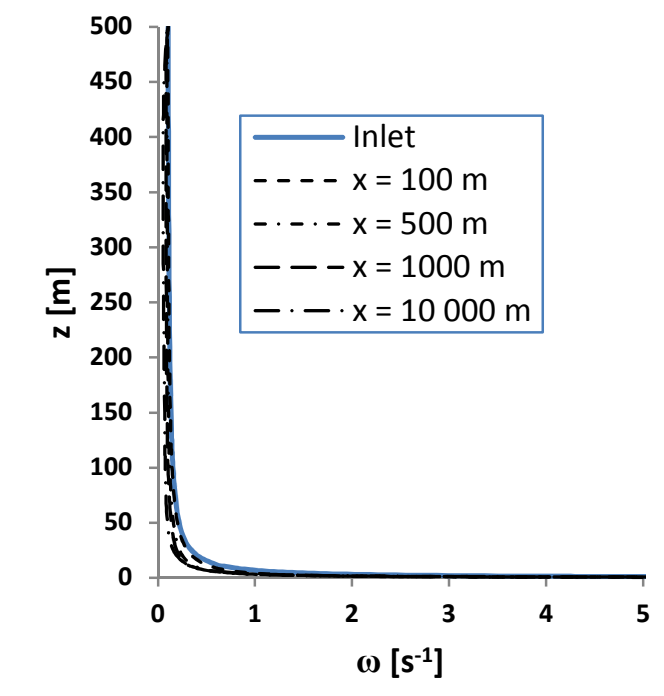

(c)

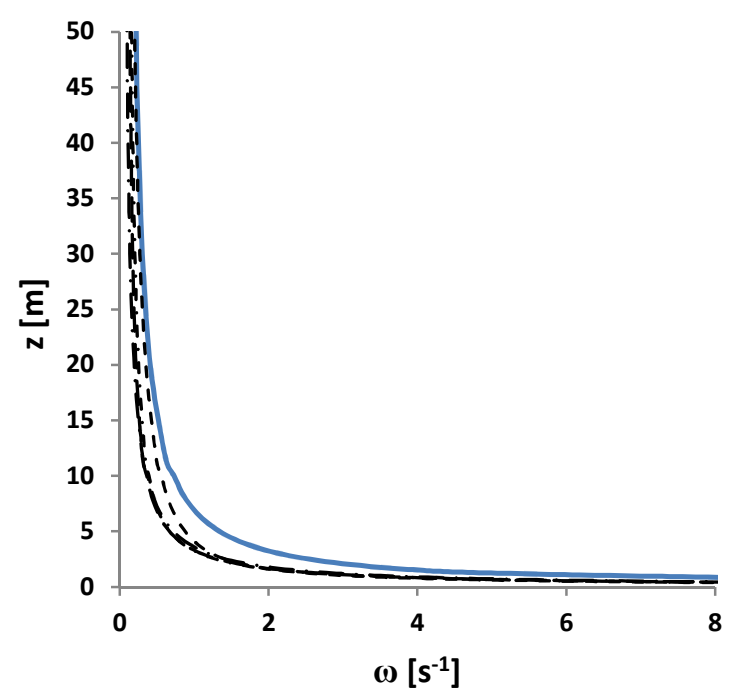

Figure F-2: Stable CFD $k$ - $\omega$ model results illustrating streamwise gradients for (a) wind speed $u$; (b) turbulent kinetic energy $k$; and (c) specific dissipation rate $\omega$. The right hand column shows the lowest $50 \mathrm{~m}$ of the domain. 

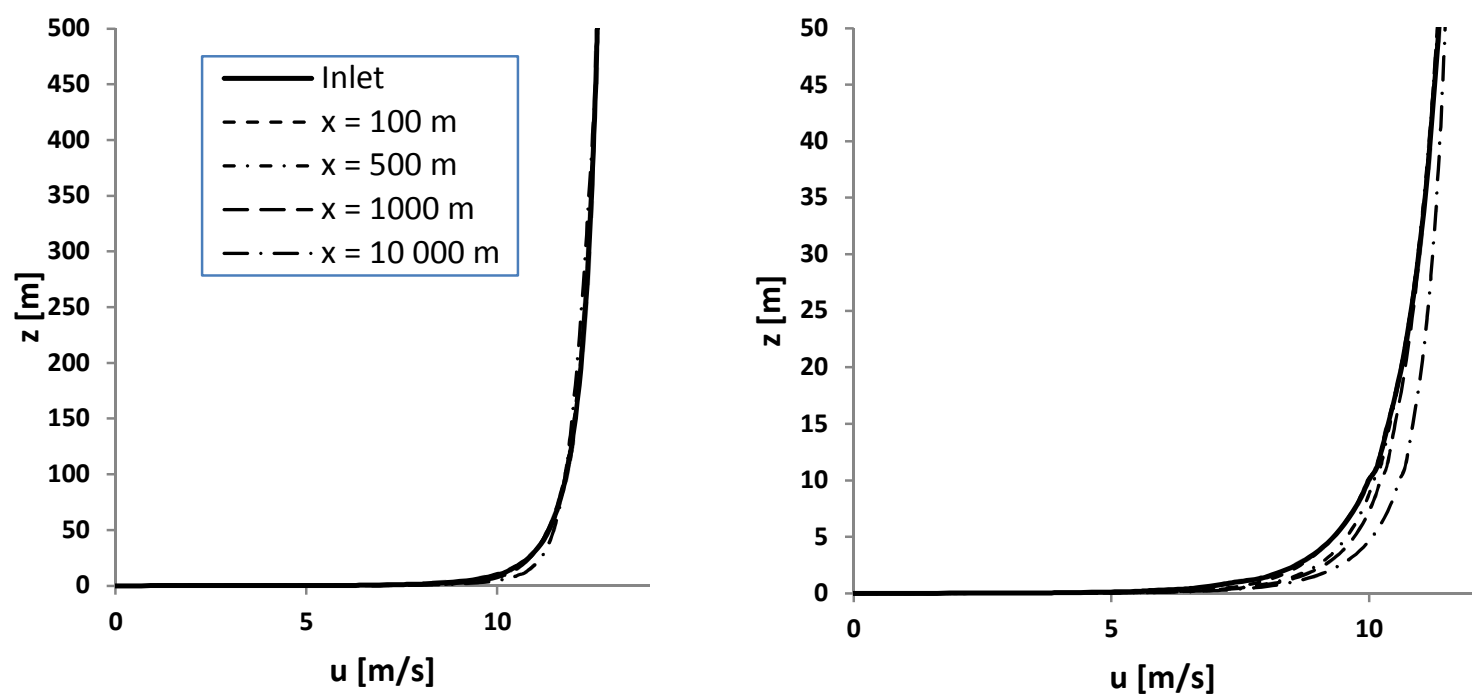

(a)
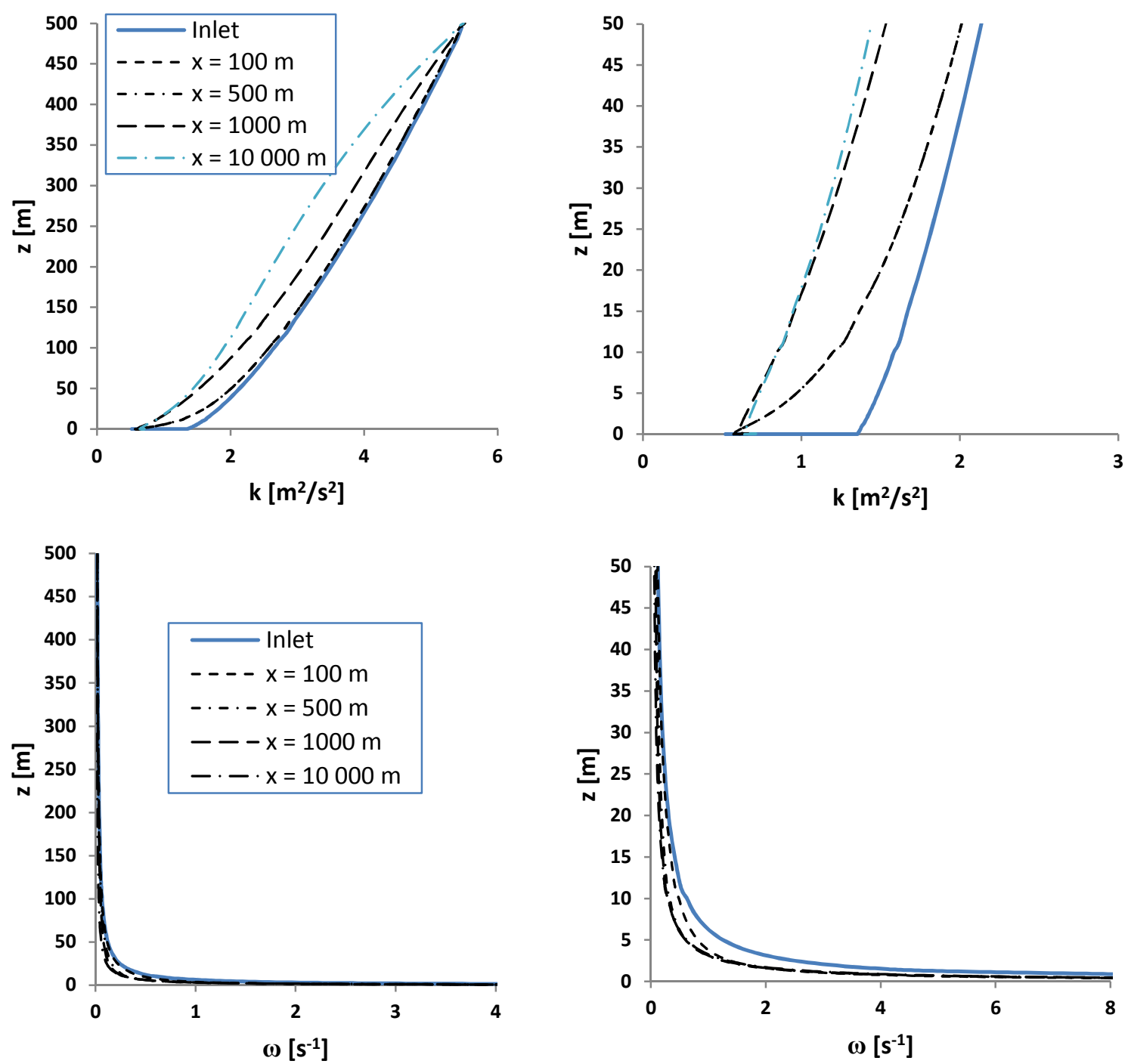

(c)

Figure F-3: Unstable CFD $k$ - $\omega$ model results illustrating streamwise gradients for (a) wind speed $u$; (b) turbulent kinetic energy $k$; and (c) specific dissipation rate $\omega$. The right hand column shows the lowest $50 \mathrm{~m}$ of the domain. 


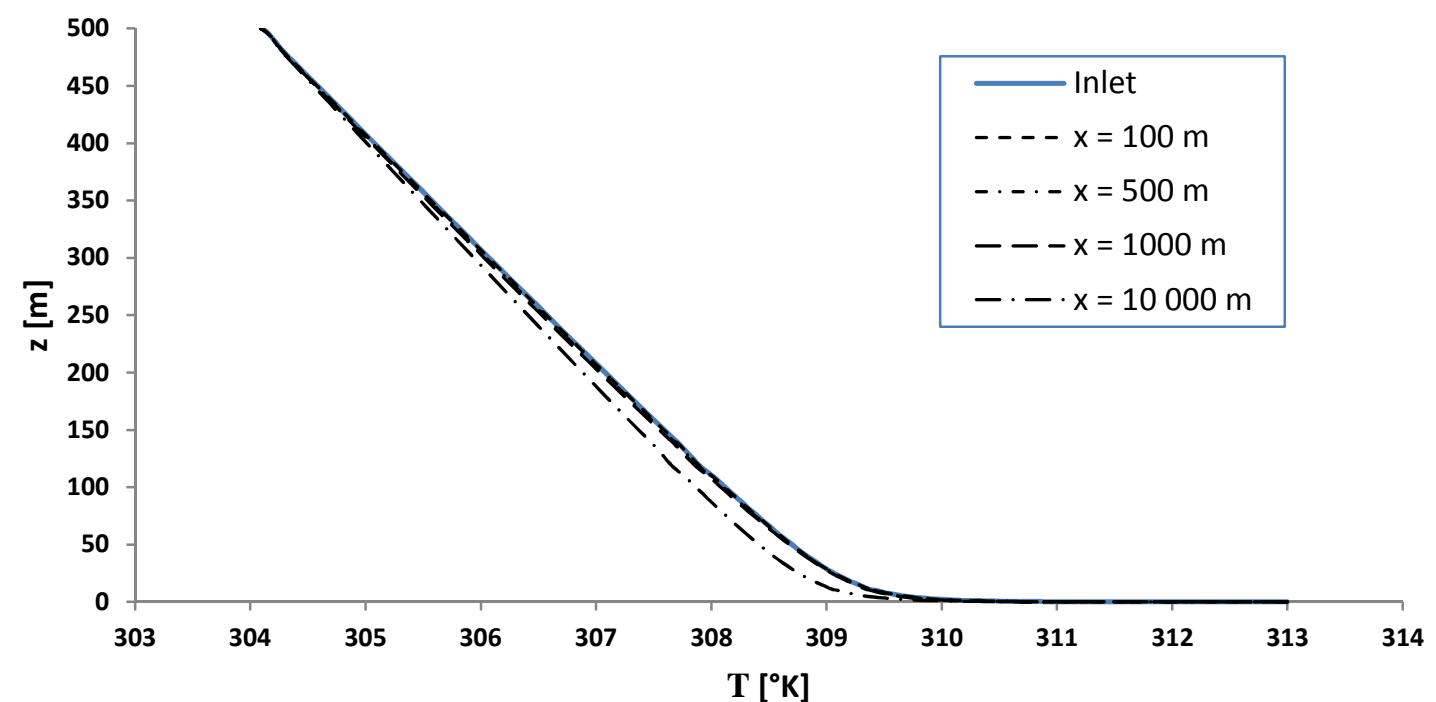

(a)

(b)

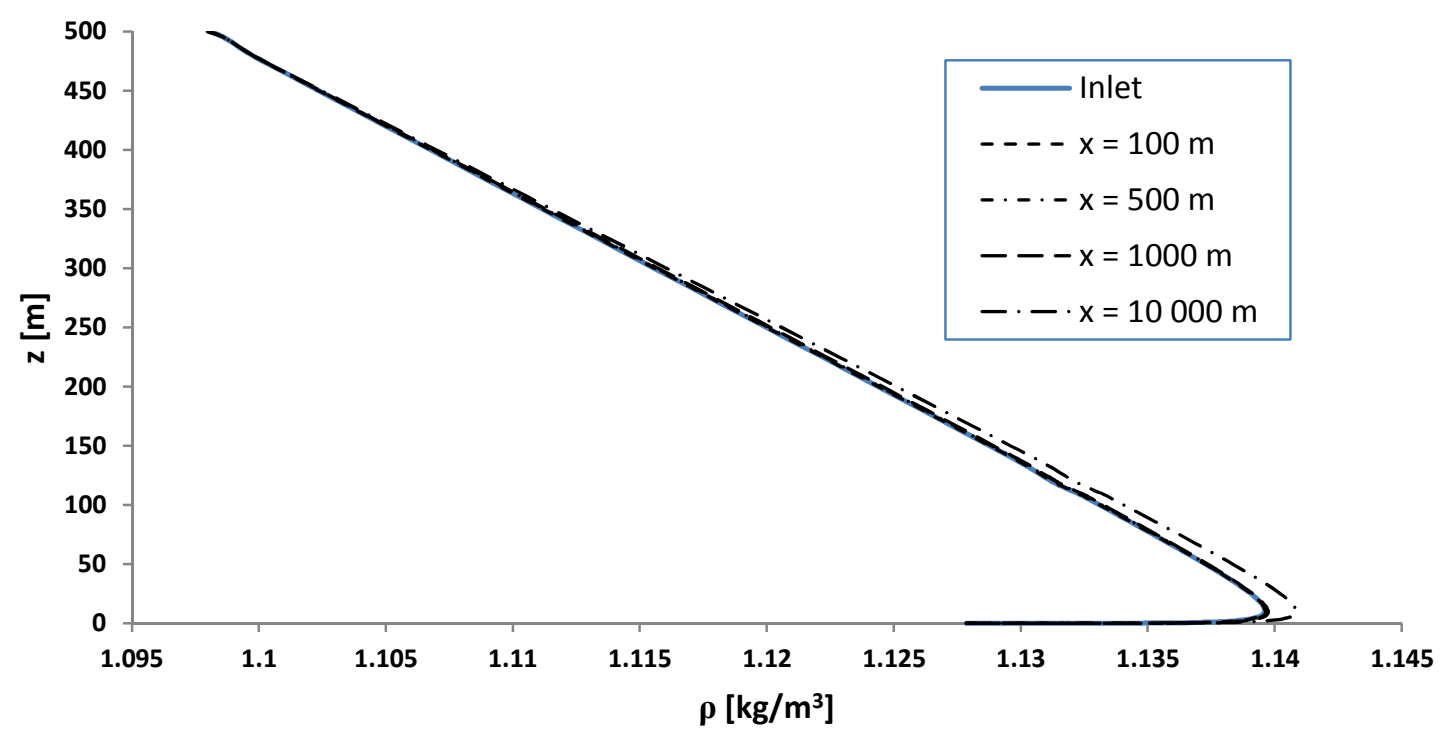

(c)

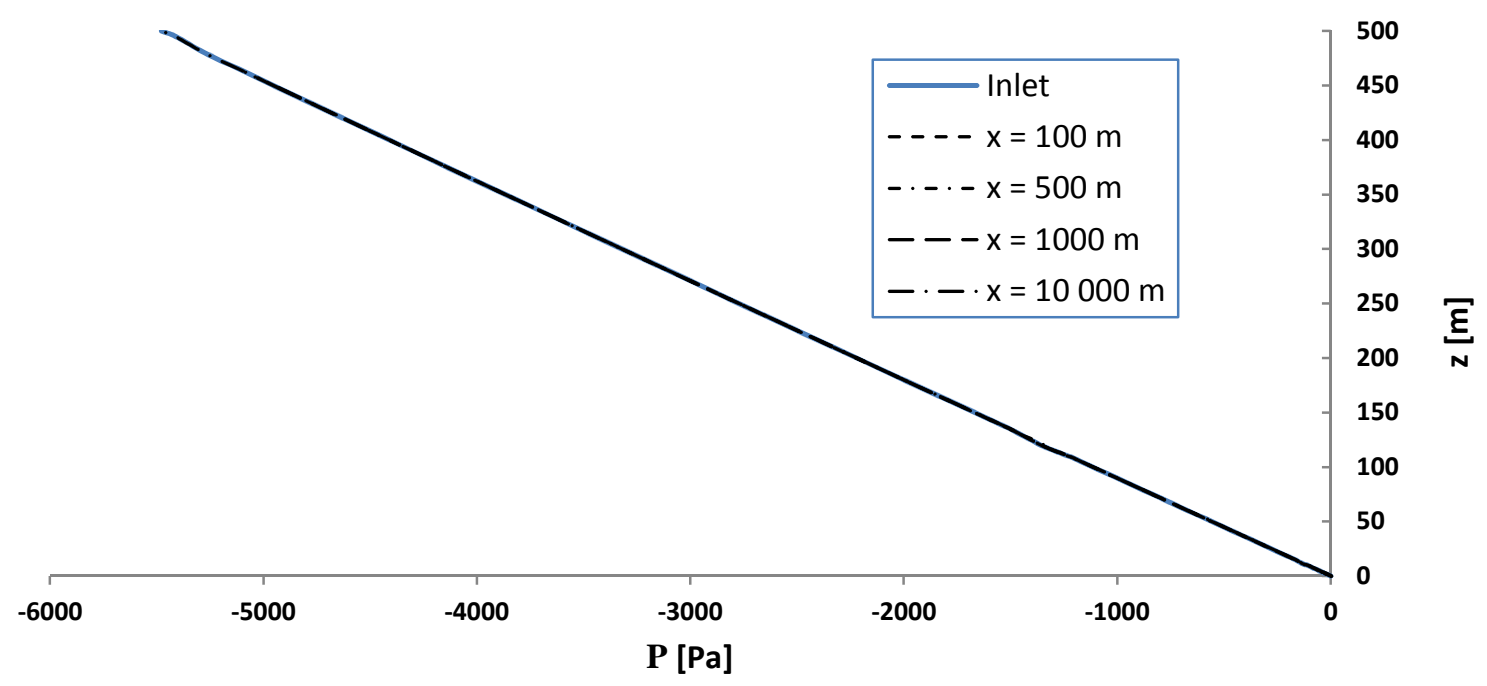

Figure F-4: Unstable CFD $k$ - $\omega$ model results illustrating streamwise gradients for (a) temperature $T$; (b) density $\rho$; and (c) pressure $P$. 


\section{APPENDIX G: RESULTS OF TURBULENT VISCOSITY FOR FLOW OVER DUNE}

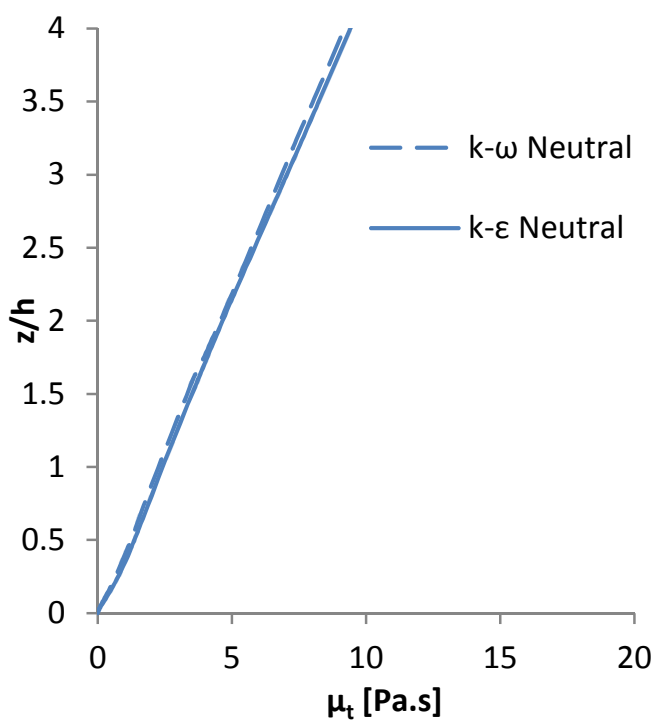

(a)

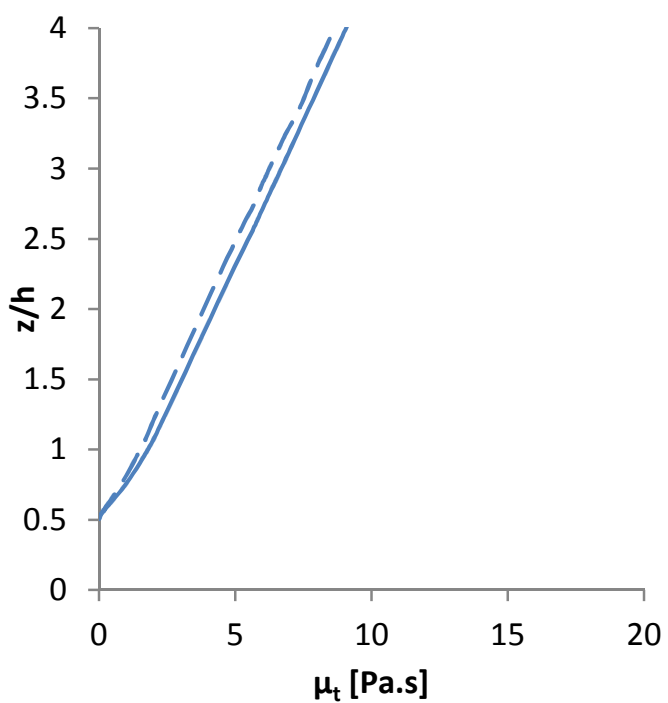

(b)

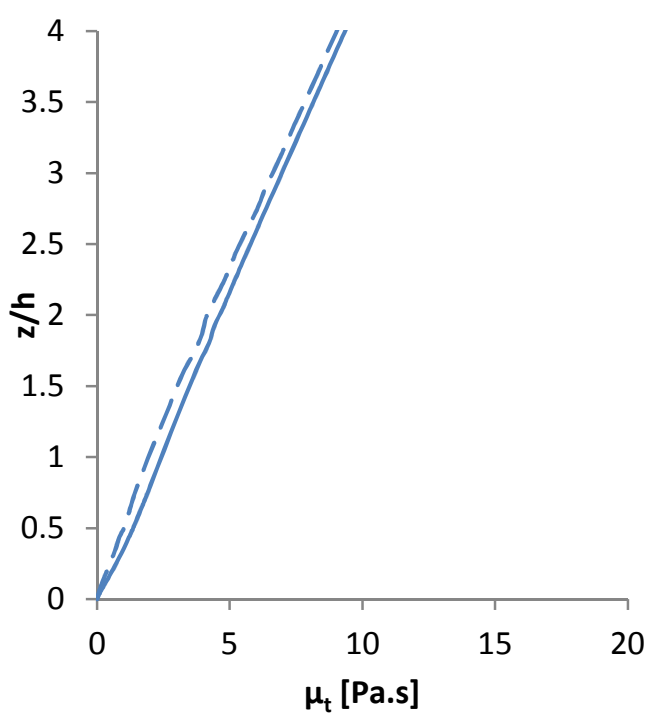

c)

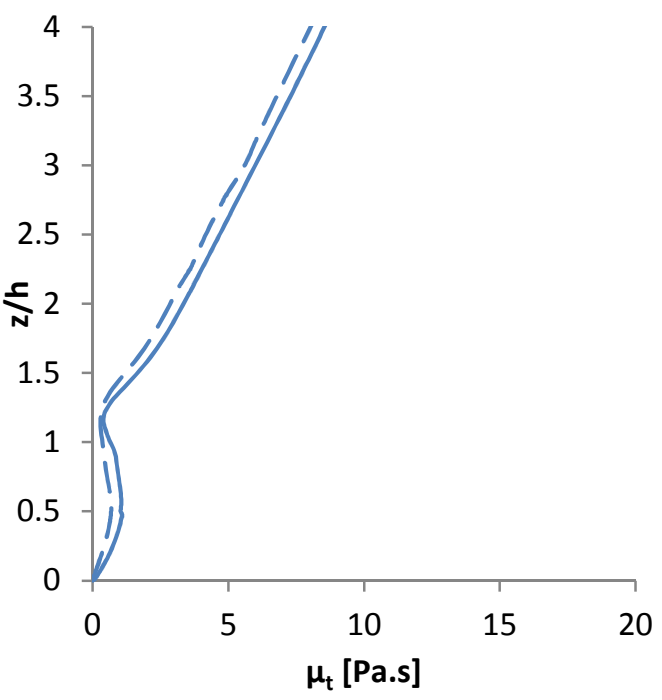

(d)
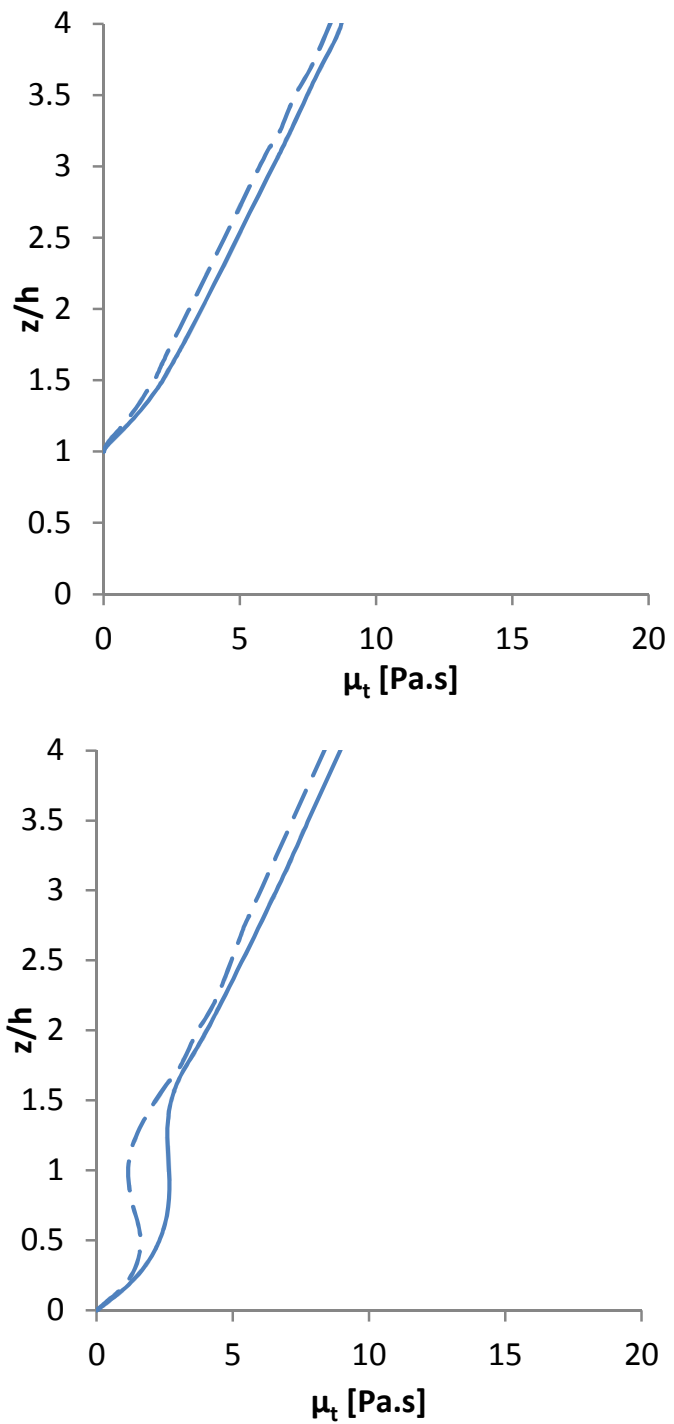

Figure G-1:Turbulent viscosity for the two turbulence models under neutral conditions at the measurement locations from $-8 h$ to $10 h$ ((a)-(f)) respectively. 

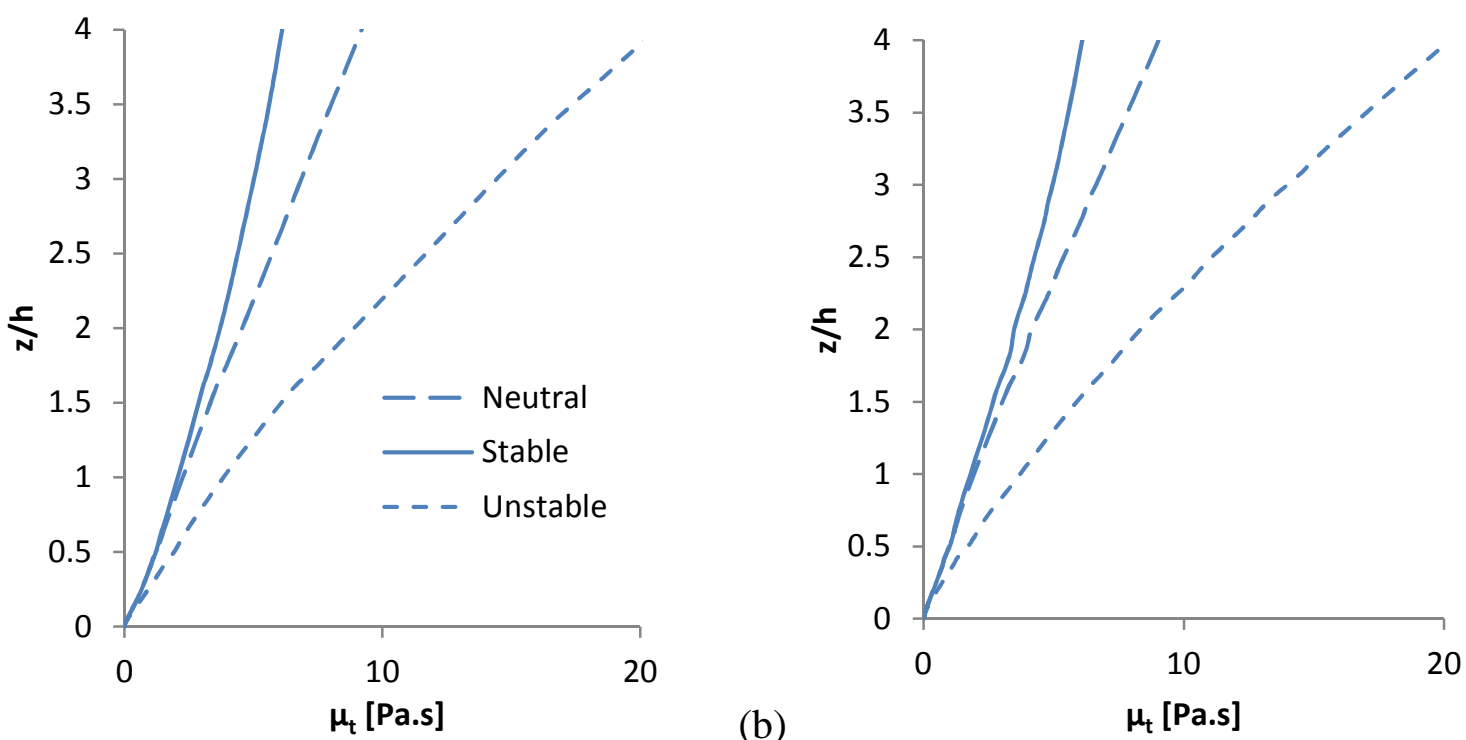

(a)

(b)

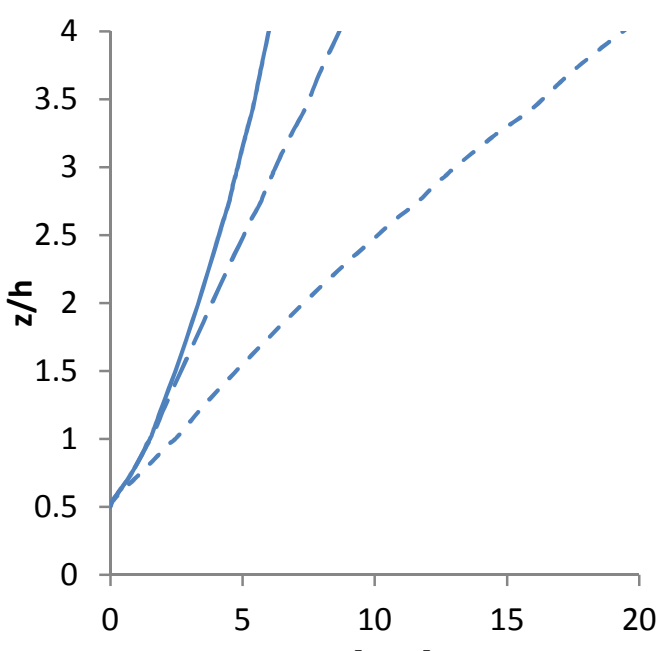

(c)

$\mu_{t}[$ Pa.s]

(d)
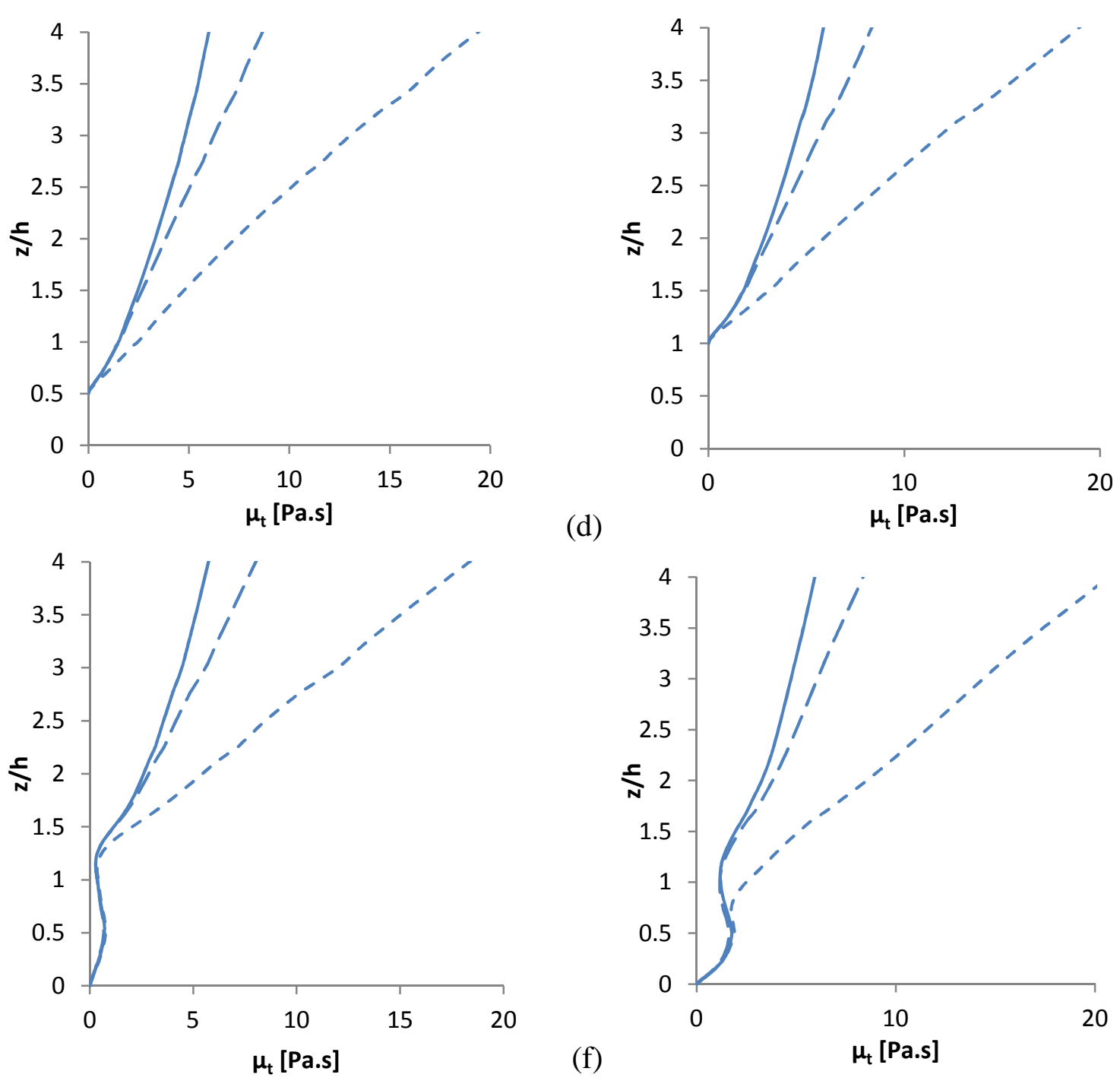

Figure G-2: Turbulent viscosity distribution for the two turbulence models for stable and unstable conditions at different locations from $-8 h$ to $10 h$ ((a)-(f)) respectively. 


\section{APPENDIX H: WIND SENSOR SPECIFICATIONS}

The calibration parameters for the sensors and further specifications of the measuring equipment used on the wind mast are tabled below as taken from Joubert (2010).

Table F-1: Sensor calibration coefficients for logger, station 1

\begin{tabular}{cccccccc}
\hline Station ID & Channel & Type & Range & Offset & Multiplier & Height [m] & Sensor ID \\
\hline Station 1 & 1 & Temp. & 6 & 0.6765 & 0.9765 & 2.5 & $1 \mathrm{~T}$ \\
& 2 & Temp. & 6 & 0.3858 & 0.9716 & 5 & $2 \mathrm{~T}$ \\
& 9 & Speed & 1 & 0.38 & 0.1537 & 2.5 & $1 \mathrm{~S}$ \\
& 10 & Speed & 1 & 0.38 & 0.1537 & 5 & $2 \mathrm{~S}$ \\
\hline
\end{tabular}

Table F-2: Sensor calibration coefficients for logger, station 2

\begin{tabular}{cccccccc}
\hline Station ID & Channel & Type & Range & Offset & Multiplier & Height [m] & Sensor ID \\
\hline Station 2 & 1 & Temp. & 6 & 1.2395 & 0.9568 & 7.5 & $3 \mathrm{~T}$ \\
& 2 & Temp. & 6 & -0.0018 & 0.9817 & 10 & $4 \mathrm{~T}$ \\
& 7 & Dir. & 8 & 0.3 & 0.992 & 6 & - \\
& 9 & Speed & 1 & 0.38 & 0.1537 & 7.5 & $3 \mathrm{~S}$ \\
& 10 & Speed & 1 & 0.38 & 0.1537 & 10 & $4 \mathrm{~S}$ \\
\hline
\end{tabular}

Table F-3: Data-logger specifications

\begin{tabular}{ll}
\hline Supplier: & MCSystems \\
Model no.: & MCS130M2 \\
Analogue Channels: & 8 \\
Digital Channels: & 2 \\
Communication: & RS232 \\
Battery: & Sealed lead acid cell, 6 Volt, $7 \mathrm{amp} / \mathrm{hr}$ \\
Housing: & IP65PVC with sealing gasket \\
\hline & \\
Table F-4: Temperature sensor specifications & \\
\hline Supplier: & MCSystems \\
Model no.: & MCS151 \\
Output: & Analogue \\
Operating temperatures range: & $-20 \mathrm{C}$ to $70 \mathrm{C}$ \\
Accuracy: & $+/-0.2 \mathrm{C}$ at $25 \mathrm{C}$ \\
Resolution: & $+/-0.1 \mathrm{C}$ \\
Power requirement: & 4.8 to 6.5 volts at $300 \mu \mathrm{A}$ \\
Dimensions: & $8 \mathrm{~mm}$ diameter $\mathrm{x} 75 \mathrm{~mm}$ \\
Mass: & $50 \mathrm{~g}$ \\
\hline
\end{tabular}


Table F-5: Wind vane specifications

\begin{tabular}{ll}
\hline Supplier: & MCSystems \\
Model no:: & MCS176 \\
Output: & Analogue \\
Operating temperatures range: & $-10 \mathrm{C}$ to $50 \mathrm{C}$ \\
Accuracy: & $+/-5$ \\
Angular Span: & 350 \\
Power Requirements: & 1 to 2.5 Volts at $200 \mu \mathrm{A}$ \\
Vane: & Anodized aluminium \\
Housing: & $\mathrm{UV}$ protected PVC \\
Vertical height: & $300 \mathrm{~mm}$ \\
Mounting requirements: & $25.4 \mathrm{~mm}$ inside diameter \\
Mass: & $500 \mathrm{~g}$ \\
\hline
\end{tabular}

Table F-6: Wind speed sensor specifications

\begin{tabular}{ll}
\hline Suppliers: & MCSystems \\
Model no.: & MCS177 \\
Output: & Digital \\
Operating wind speed range: & 0.5 to $45 \mathrm{~m} / \mathrm{s}$ \\
Operating temperatures range: & $-10 \mathrm{C}$ to $50 \mathrm{C}$ \\
Accuracy: & $+2 \%$ full scale \\
Turning radius: & $150 \mathrm{~mm}$ \\
Cup size: & $70 \mathrm{~mm}$ diameter \\
Cups: & Anodized aluminium \\
Power requirement: & 4 to 15 Volts at $20 \mu \mathrm{A}$ \\
Vane: & Anodized aluminium \\
Housing: & UV protected PVC \\
Vertical height: & $300 \mathrm{~mm}$ \\
Mounting requirements: & $25.4 \mathrm{~mm}$ inside diameter \\
Mass: & $0.5 \mathrm{~kg}$ \\
\hline
\end{tabular}




\section{REFERENCES}

Alinot, C. \& Masson, C. 2002, "Aerodynamic Simulations of Wind Turbines Operating in Atmospheric Boundary Layer With Various Thermal Stratifications", ASME Conference Proceedings, vol. 2002, no. 7476X, pp. 206-215.

Alinot, C. \& Masson, C. 2005, "k- $\varepsilon$ Model for the Atmospheric Boundary Layer Under Various Thermal Stratifications", Journal of Solar Energy Engineering, vol. 127, no. 4, pp. 438-443.

Arya, S.P. 2001a, "Chapter 10 Near-neutral boundary layers" in International Geophysics, ed. H. Holton, Academic Press, New York, pp. 188-212.

Arya, S.P. 2001b, "Chapter 11 Thermally stratified surface layer" in International Geophysics, ed. H. Holton, Academic Press, New York, pp. 213-241.

Arya, S.P. 2001c, "Chapter 5 Air temperature and humidity in the PBL" in International Geophysics, ed. H. Holton, Academic Press, New York, pp. 6288.

Arya, S.P. 2005, "Micrometeorology and Atmospheric Boundary Layer", Pure and Applied Geophysics, vol. 162, no. 10, pp. 1721-1745.

Azad, R.S. 1993, The Atmospheric Boundary Layer for Engineers, Kluwer, Dordrecht.

Balogh, M., Parente, A. \& Benocci, C. 2012, "RANS Simulation of ABL Flow over Complex Terrains Applying an Enhanced $k-\varepsilon$ Model and Wall Function Formulation: Implementation and Comparison for Fluent and OpenFOAM", Journal of Wind Engineering and Industrial Aerodynamics, vol. 104, no. 106, pp. 360-368.

Blackadar, A.K. 1997, Turbulence and Diffusion in the Atmosphere, SpringerVerlag, Berlin.

Blocken, B., Carmeliet, J. \& Stathopoulos, T. 2007a, "CFD Evaluation of Wind Speed Conditions in Passages Between Parallel Buildings- Effect of WallFunction Roughness Modifications for the Atmospheric Boundary Layer Flow", Journal of Wind Engineering and Industrial Aerodynamics, vol. 95, no. 9-11, pp. 941-962.

Blocken, B., Stathopoulos, T. \& Carmeliet, J. 2007b, "CFD Simulation of the Atmospheric Boundary Layer: Wall Function Problems", Atmospheric Environment, vol. 41, no. 2, pp. 238-252. 
Blocken, B., Janssen, W. \& van Hooff, T. 2011, "CFD Simulation for Pedestrian Wind Comfort and Wind Safety in Urban Areas: General Decision Framework and Case Study for the Eindhoven University Campus", Environmental Modelling \& Software, vol. 30, no. 1, pp. 15-34.

Bristow, C., Bailey, S. \& Lancaster, N. 2000, "The Sedimentary Structure of Linear Sand Dunes", Nature, vol. 406, no. 6791, pp. 56-59.

Britter, R. \& Schatzmann, M. 2007, "Model Evaluation Guidance and Protocol Document", COST Office Brussels.

Businger, J.A., Wyngaard, J., Izumi, Y. \& Bradley, E. 1971, "Flux-profile Relationships in the Atmospheric Surface Layer", Journal of the Atmospheric Sciences, vol. 28, no. 2, pp. 181-189.

CD-adapco Inc. 2011, STAR-CCM+ Version 6.02.007 User Guide, CD-adapco Inc., New York.

Celik, I.B., Ghia, U. \& Roache, P.J. 2008, "Procedure for Estimation and Reporting of Uncertainty due to Discretization in CFD Applications", Journal of Fluids Engineering-Transactions of the ASME, vol. 130, no. 7.

Dyer, A. 1974, "A Review of Flux-profile Relationships", Boundary-Layer Meteorology, vol. 7, no. 3, pp. 363-372.

Ferreira, A., Lopes, A., Viegas, D. \& Sousa, A. 1995, "Experimental and Numerical Simulation of Flow Around Two-Dimensional Hills", Journal of Wind Engineering and Industrial Aerodynamics, vol. 54, pp. 173-181.

Fluent Inc. 2006. Fluent 6.2 User's Guide. Fluent Inc., Lebanon.

Franke, J., Hellsten, A., Schlunzen, K.H. \& Carissimo, B. 2011, "The COST 732 Best Practice Guideline for CFD Simulation of Flows in the Urban Environment: a Summary", International Journal of Environment and Pollution, vol. 44, no. 1, pp. 419-427.

Garratt, J. 1994, "Review: the Atmospheric Boundary Layer", Earth-Science Reviews, vol. 37, no. 1, pp. 89-134.

Griffiths, A. \& Middleton, J. 2010, "Simulations of Separated Flow over TwoDimensional Hills", Journal of Wind Engineering and Industrial Aerodynamics, vol. 98, no. 3, pp. 155-160.

Hargreaves, D. \& Wright, N. 2007, "On the Use of the $k-\varepsilon$ Model in Commercial CFD Software to Model the Neutral Atmospheric Boundary Layer", Journal 
of Wind Engineering and Industrial Aerodynamics, vol. 95, no. 5, pp. 355369.

Huser, A., Nilsen, P.J. \& Skåtun, H. 1997, "Application of k-E Model to the Stable ABL: Pollution in Complex Terrain", Journal of Wind Engineering and Industrial Aerodynamics, vol. 67-68, no. 0, pp. 425-436.

Joubert, E.C. 2010, A Computational Fluid Dynamics Study of the Near Surface Wind Patterns over a Desert Dune and the Effect on Seed Dispersion. A master of science engineering thesis, Department of Mechanical Engineering, University of Stellenbosch, South Africa.

Joubert, E.C., Harms, T.M., Muller, A., Hipondoka, M. \& Henschel, J.R. 2012, "A CFD Study of Wind Patterns over a Desert Dune and the Effect on Seed Dispersion", Environmental Fluid Mechanics, vol. 12, no. 1, pp. 23-44.

Kim, S.E. \& Boysan, F. 1999, "Application of CFD to Environmental Flows", Journal of Wind Engineering and Industrial Aerodynamics, vol. 81, no. 1, pp. 145-158.

Kitada, T. 1987, "Turbulence Structure of Sea Breeze Front and its Implication in Air Pollution Transport - Application of $k-\varepsilon$ Turbulence Model", BoundaryLayer Meteorology, vol. 41, no. 1, pp. 217-239.

Kristóf, G., Rácz, N. \& Balogh, M. 2009, "Adaptation of Pressure Based CFD Solvers for Mesoscale Atmospheric Problems", Boundary-Layer Meteorology, vol. 131, no. 1, pp. 85-103.

Kröger, D.G. 2004, Air-cooled Heat Exchangers and Cooling Towers: Thermalflow Performance Evaluation and Design, Penwell Corporation.

Launder, B.E. \& Spalding, D. 1974, "The Numerical Computation of Turbulent Flows", Computer Methods in Applied Mechanics and Engineering, vol. 3, no. 2, pp. 269-289.

Liu, B., Qu, J., Zhang, W. \& Qian, G. 2011, "Numerical Simulation of Wind Flow over Transverse and Pyramid Dunes", Journal of Wind Engineering and Industrial Aerodynamics, vol. 99, no. 8, pp. 879-888.

Livingstone, I., Wiggs, G.F.S. \& Weaver, C.M. 2007, "Geomorphology of Desert Sand Dunes: a Review of Recent Progress", Earth-Science Reviews, vol. 80, no. 3, pp. 239-257.

Lumley, J.S.P. 1964, The Structure of Atmospheric Turbulence, WileyInterscience, New York. 
Meissner, C., Gravdahl, A.R. \& Steensen, B. 2009, "Including Thermal Effects in CFD Simulations", European Wind Energy Conference and Exhibition, 2009.

Mills, A.F. 1999, Heat Transfer, Prentice Hall, Upper Saddler River, NJ.

Parente, A., Gorlé, C., van Beeck, J. \& Benocci, C. 2011a, "A Comprehensive Modelling Approach for the Neutral Atmospheric Boundary Layer: Consistent Inflow Conditions, Wall Function and Turbulence Model", Boundary-Layer Meteorology, vol. 140, no. 3, pp. 411-428.

Parente, A., Gorlé, C., van Beeck, J. \& Benocci, C. 2011b, "Improved $k-\varepsilon$ Model and Wall Function Formulation for the RANS Simulation of ABL Flows", Journal of Wind Engineering and Industrial Aerodynamics, vol. 99, no. 4, pp. 267-278.

Parsons, D.R., Walker, I.J. \& Wiggs, G.F.S. 2004a, "Numerical Modelling of Flow Structures over Idealized Transverse Aeolian Dunes of Varying Geometry", Geomorphology, vol. 59, no. 1, pp. 149-164.

Parsons, D.R., Wiggs, G.F.S., Walker, I.J., Ferguson, R.I. \& Garvey, B.G. 2004b, "Numerical Modelling of Airflow over an Idealised Transverse Dune", Environmental Modelling \& Software, vol. 19, no. 2, pp. 153-162.

Peng, S.H. \& Davidson, L. 1999, "Computation of Turbulent Buoyant Flows in Enclosures with Low-Reynolds-number $k$ - $\omega$ Models", International Journal of Heat and Fluid Flow, vol. 20, no. 2, pp. 172-184.

Pontiggia, M., Derudi, M., Busini, V. \& Rota, R. 2009, "Hazardous Gas Dispersion: A CFD model Accounting for Atmospheric Stability Classes", Journal of Hazardous Materials, vol. 171, no. 1, pp. 739-747.

Richards, P. \& Hoxey, R. 1993, "Appropriate Boundary Conditions for Computational Wind Engineering Models Using the $k-\epsilon$ Turbulence Model", Journal of Wind Engineering and Industrial Aerodynamics, vol. 46, no. 47, pp. 145-153.

Rodi, W. 1997, "Comparison of LES and RANS Calculations of the Flow Around Bluff Bodies", Journal of Wind Engineering and Industrial Aerodynamics, vol. 69, no. 71, pp. 55-75.

Takahashi, T., Kato, S., Murakami, S., Ooka, R., Fassy Yassin, M. \& Kono, R. 2005, "Wind Tunnel Tests of Effects of Atmospheric Stability on Turbulent Flow over a Three-dimensional Hill", Journal of Wind Engineering and Industrial Aerodynamics, vol. 93, no. 2, pp. 155-169.

TheMathWorks Inc. 2010, MATLAB version 7.1, Natick, MA. 
Thomas, D.S.G., Knight, M. \& Wiggs, G.F.S. 2005, "Remobilization of Southern African Desert Dune Systems by Twenty-first Century Global Warming", Nature, vol. 435, no. 7046, pp. 1218-1221.

Uehara, K., Wakamatsu, S. \& Ooka, R. 2003, "Studies on Critical Reynolds Number Indices for Wind-tunnel Experiments on Flow within Urban Areas", Boundary-Layer Meteorology, vol. 107, no. 2, pp. 353-370.

Versteeg, H.K. \& Malalasekera, W. 2007, An Introduction to Computational Fluid Dynamics: the Finite Volume Method, Prentice Hall, Malaysia.

White, F.M. 1991, Viscous Fluid Flow, McGraw-Hill, New York.

Wieringa, J. 2001, "New Revision of Davenport Roughness Classification", 3rd European \& African Conference on Wind Engineering, July 2001, pp. 285.

Wiernga, J. 1993, "Representative Roughness Parameters for Homogeneous Terrain", Boundary-Layer Meteorology, vol. 63, no. 4, pp. 323-363.

Wiggs, G.F.S., Livingstone, I. \& Warren, A. 1996, "The Role of Streamline Curvature in Sand Dune Dynamics: Evidence From Field and Wind Tunnel Measurements", Geomorphology, vol. 17, no. 1, pp. 29-46.

Yang, W., Quan, Y., Jin, X., Tamura, Y. \& Gu, M. 2008, "Influences of Equilibrium Atmosphere Boundary Layer and Turbulence Parameter on Wind Loads of Low-rise Buildings", Journal of Wind Engineering and Industrial Aerodynamics, vol. 96, no. 10, pp. 2080-2092. 